NASA Contractor Report 3613

\title{
Jet Noise Suppression by Porous Plug Nozzles
}

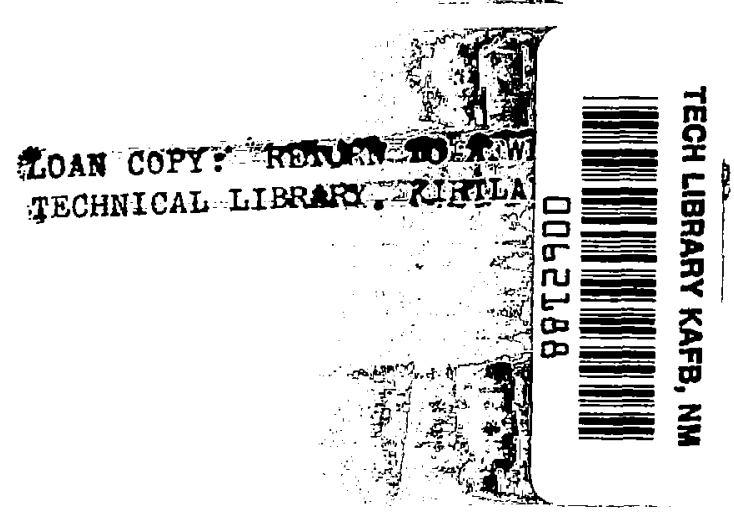

A. B. Bauer, V. Kibens, and R. W. Wlezien

CONTRACT NAS 1-16284

OCTOBER 1982

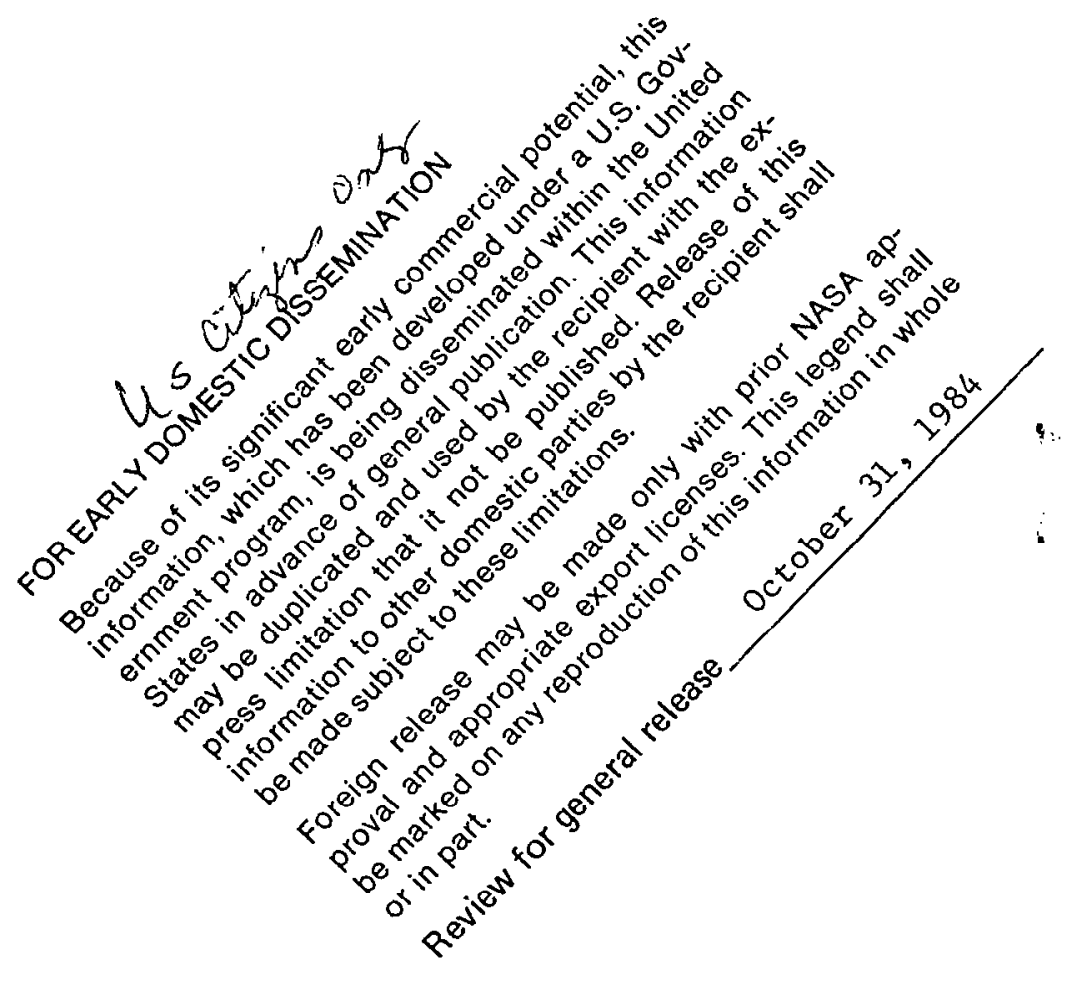


GED DOCUMENT

Note that this document bears the label "FEDD," an acronym for "FOR EARLY DOMESTIC DISSEMINATION." The FEDD label is affixed to documents that may contain information having high commercial potential.

The FEDD concept was developed as a result of the desire to maintain U.S. leadership in world trade markets and encourage a favorable balance of trade. Since the availability of taxsupported U.S. technology to foreign business interests could represent an unearned benefit, research results that may have high commercial potential are being distributed to U.S. industry in advance of general release.

The recipient of this report must treat the information it contains according to the conditions of the FEDD label on the front cover.

$19 t^{i}$ origins anvounal in STAR NASA Hops. 
NASA Contractor Report 3613

\title{
Jet Noise Suppression by Porous Plug Nozzles
}

\author{
A. B. Bauer \\ Douglas Aircraft Company \\ Long Beach, California \\ V. Kibens and R. W. Wlezien \\ McDonnell Douglas Research Laboratories \\ St. Louis, Missouri
}

Prepared for

Langley Research Center

under Contract NAS1-16284

\section{N/Sก}

National Aeronautics

and Space Administration

Scientlfic and Technical

Information Branch

1982 

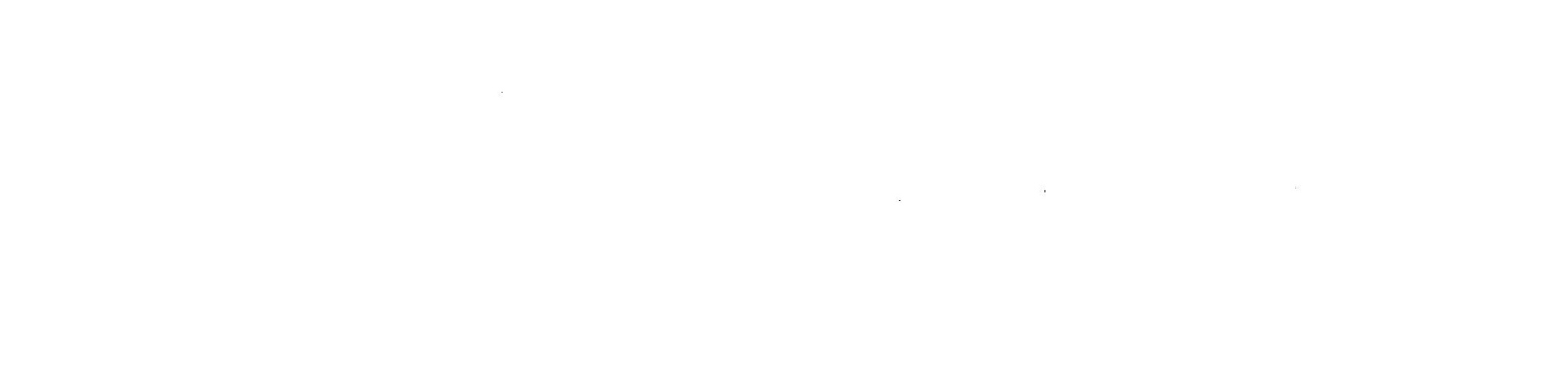


\section{CONTENTS}

Page

INTRODUCTION...................................... 1

SYMBOLS........................................... 3

MODEL TEST PROGRAM............................... 5

Mode1 Configurations............................. 5

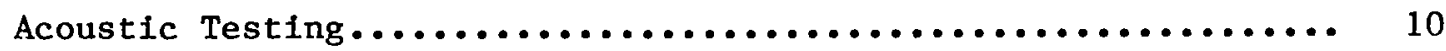

Test $\operatorname{setup} . \ldots \ldots \ldots \ldots \ldots \ldots \ldots \ldots \ldots \ldots \ldots \ldots \ldots \ldots \ldots \ldots \ldots \ldots . \ldots \ldots$

Test Precautions................................. 12

Test Conditions................................ 13

Aerodynamic Testing............................. 17

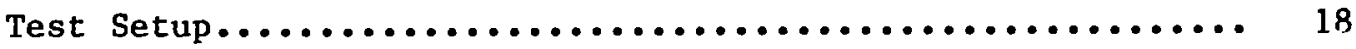

Laser Doppler Velocimeter System.................. 21

Static Pressure Measurements....................... 25

Microphone Measurements.......................... 26

Flow Visualization........................... 27

TEST RESULTS................................... 28

Acoustic Test Results............................. 28

Cross-Correlation Measurements..................... 28

Jet Noise Suppression with Cold Flow................. 32

Acoustic Shield Effects........................... 46

Jet Noise Suppression with Hot Flow................. 47

Forward-Motion Flow Effects...................... 49

Tradeoff Between Thrust and Noise Reduction.............. 51

Aerodynamic Test Results........................... 57

Correlation of Pressure and Velocity Measurements......... 58

Summary of Flowfield Measurements................... 65

Scale of Pressure Variations...................... 73

Near-Field Acoustic Results...................... 78

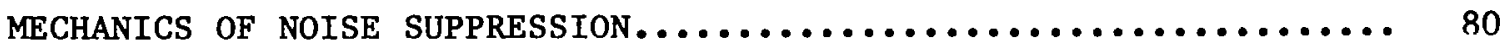

CONCLUSIONS..................................... 88

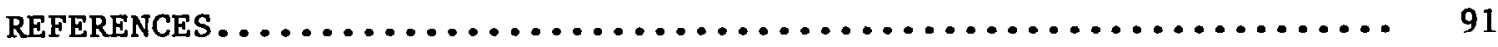

APPENDIX A - SOUND PRESSURE LEVEL AS A FUNCTION OF ONE-THIRD

OCTAVE BAND FREQUENCY ............................ 93

APPENDIX B - SUPPLEMENTARY AERODYNAMIC TEST RESULTS............ 130

APPENDIX $C$ - THRUST EFFICIENCY.......................... 165

APPENDIX D - EFFECTS OF FORWARD MOTION ON JET NOISE........... 168 


\section{INTRODUCTION}

Jet noise can be suppressed significantly by the use of an axisymmetric and porous body, known as a plug, mounted along the centerline of the jet exhaust nozzle and in the high-speed portion of the exhaust plume. The porous plug concept has been under development by Maestrello of NASA (References 1 and 2), but the idea is so new that his results were the only experimental data avallable prior to the present work. Furthermore, although Maestrel1o's results show jet noise reductions of 5 to $15 \mathrm{~dB}$, depending on the jet Mach number as well as several other parameters, the theory of the porous plug concept is in such a primitive state that these noise reductions are not predictable. Therefore, the present program was initiated to obtain a wide background of experimental results from plug suppressors of various geometric shapes to provide a better understanding of the mechanisms of porous plug operation.

Prior work in jet noise suppression has included several fundamental approaches, among which are those involving 1) a decrease in the mean jet velocity, 2) a modification of the jet temperature and velocity profiles, and 3) devices that shield or absorb the noise. The first method has been implemented by the design of high bypass-ratio engines during the past 20 years, and has produced fuel economies as well as large jet noise reductions. An example of the second method is the mixer nozzle which on some modern engines can give a moderate noise reduction with no significant loss in engine thrust and fuel economy, sometimes producing a gain in efficiency. A mixer nozzle combined with a shroud can produce large reductions in jet noise, however, at the expense of additional weight and complex retraction mechanisms required to avoid a substantial cruise drag penalty. The porous plug does not significantly alter the mean velocity or the temperature and velocity profiles at the nozzle exhaust plane, but modifies the jet velocity downstream of the exhaust plane so as to reduce shock strength and shock cell noise. The porous plug also acts to shield and absorb some of the noise.

The tests reported here are the first porous plug suppressor results for which the thrust has been measured directly, although 
Maestrello has measured the thrust by means of the impingement of the jet on a flat plate. The thrust measurements are used to determine the propulsive efficiency of the suppressor nozzles. The propulstve results are presented with the jet noise measurements so that relations between the noise reductions and the thrust efficiency are obtained. Effects of hot primary flow and forward motion flow are reported.

The physical mechanisms that lead to the sound reductions have been examined by a set of aerodynamic measurements of both standard jet and porous plug jet flowfields. Extensive results obtained by laser Doppler velocity measurements, static pressure measurements, shadowgraph photography, and shadowgraph cinematography have been correlated to show the role of the porous plug in controlling shock patterns and shock noise. 


\section{SYMBOLS}

\begin{tabular}{|c|c|}
\hline $\mathrm{C}_{\mathrm{D}}$ & nozzle discharge coefficient \\
\hline$c_{\mathrm{f}}$ & skin friction coefficient \\
\hline $\mathrm{C}_{\mathrm{T}}$ & nozzle thrust coefficient \\
\hline $\mathrm{C}_{\mathrm{Tf}}$ & boundary-layer thrust coefficient loss \\
\hline $\mathrm{C}_{\mathrm{Th}}$ & hole thrust coefficient loss \\
\hline $\mathrm{C}_{\mathrm{Tn}}$ & flow-nonuniformity thrust coefficient loss \\
\hline $\mathrm{C}_{\mathrm{Ts}}$ & strut thrust coefficient loss \\
\hline $\mathrm{C}_{\mathrm{TPR}}$ & thrust coefficient at a specified pressure ratio \\
\hline d & diameter of equivalent circular nozzle \\
\hline$d_{0}$ & diameter of plug at exhaust plane \\
\hline $\mathrm{d}_{1}$ & diameter of nozzle at exhaust plane \\
\hline f & frequency \\
\hline $\mathrm{f}_{1 / 3}$ & 1/3-octave-band-center frequency \\
\hline $\mathrm{f}_{\mathrm{e}}$ & modified band-center frequency \\
\hline $\mathrm{f}_{\mathrm{f}}$ & band-center frequency with forward motion \\
\hline$f_{1}$ & ideal band-center frequency \\
\hline h & exit height, $d_{1}-d_{0}$ \\
\hline $\mathbf{k}_{\mathbf{h}}$ & hole thrust loss coefficient \\
\hline $\mathrm{L}_{\mathrm{n}}$ & length of pressure cel1 number $n$ \\
\hline$\ell$ & length of plug from exhaust plane \\
\hline$\ell_{c}$ & length of constant diameter portion of plug \\
\hline$\ell_{e}$ & effective length of nozzle or plug \\
\hline$\dot{\mathrm{m}}_{1}$ & ideal one-dimenstonal mass flow rate \\
\hline $\mathrm{m}_{\mathrm{m}}$ & measured mass flow rate \\
\hline M & Mach number \\
\hline$M^{*}$ & fully expanded Mach number \\
\hline $\mathrm{M}_{\mathrm{f}}$ & forward-motion flow Mach number \\
\hline $\mathbf{n}$ & ce11 number \\
\hline$P_{\mathbf{a}}$ & atmospheric pressure \\
\hline$P_{1}$ & pressure before compression wave \\
\hline $\mathrm{P}_{2}$ & pressure after compression wave \\
\hline $\mathbf{r}$ & radial coordinate \\
\hline St & Strouhal number \\
\hline+ & plug wall thickness \\
\hline
\end{tabular}




$\begin{array}{ll}\mathrm{T}_{\mathrm{m}} & \text { measured thrust } \\ \mathrm{T}_{\mathrm{o}} & \text { ambient temperature } \\ \mathrm{u} & \text { axial velocity deviation from mean } \\ \mathbf{u}^{\prime} & \text { root-mean-square of axial velocity deviation } \\ \overline{u v} & \text { Reynolds stress } \\ \mathrm{U} & \text { axial velocity component } \\ \mathrm{U}_{\mathrm{e}} & \text { modified jet velocity } \\ \mathrm{U}_{\mathrm{f}} & \text { forward flow velocity } \\ \mathrm{U}_{\mathrm{i}} & \text { ideal jet velocity } \\ \mathrm{U}_{\mathrm{ref}} & \text { jet reference velocity } \\ \mathrm{v} & \text { radial velocity deviation from mean } \\ \mathrm{v}^{\prime} & \text { root-mean-square of radial velocity deviation } \\ \mathrm{V} & \text { radial velocity component } \\ \mathrm{x} & \text { axial coordinate } \\ \mathrm{X}_{\mathrm{P}} & \text { axial location of microphone reference point } \\ \mathrm{Y}_{\mathrm{p}} & \text { radial location of microphone reference point } \\ \delta & \text { angle between wall and jet axis } \\ \gamma & \text { ratio of specific heats } \\ \sigma & \text { porosity of plug surface } \\ \theta & \text { acoustic emission angle } \\ & \end{array}$


MODEL TEST PROGRAM

The model test program has been carried out using a $14 \%$ scale model of the General Electric CF6-50 engine primary exhaust system. The standard CF6 exhaust plug was replaced with porous plug suppressor configurations, and a number of new exhaust nozzles were also constructed.

\section{Model Configurations}

The ten model configurations are described in Table 1 and illustrated by Figures 1-4. Figure 1 illustrates the complete flow system for one of the configurations. The CF6 primary exhaust system is modeled by the nozzle and the plug support assembly that uses eight

TABLE 1. GEOMETRIC PARAMETERS OF TEST CONFIGURATIONS.

\begin{tabular}{llccccc} 
Configuration $\dagger$ & $\mathbf{d}_{\mathbf{1}} / \mathbf{d}$ & $\mathbf{d}_{\mathbf{o}} / \mathbf{d}_{\mathbf{1}}$ & $\ell_{\mathbf{c}} / \mathbf{d}$ & $\ell / \mathbf{d}$ & $\sigma$ & $\mathrm{t} / \mathrm{d}$ \\
\hline $1,1^{*}$ & 1.0 & 0 & 0 & 0 & 0 & \\
2 & 1.0 & 0 & 0 & 0 & 0 & \\
3 & 1.15 & 0.50 & 0 & 0.60 & 0 & \\
$4,4^{*}$ & 1.18 & 0.53 & 2.0 & 3.45 & 0.027 & 0.0065 \\
5 & 1.18 & 0.53 & 2.0 & 3.45 & 0.056 & 0.0065 \\
6 & 1.18 & 0.53 & 2.0 & 3.45 & 0.027 & 0.0194 \\
7 & 1.18 & 0.53 & 3.0 & 4.45 & 0.027 & 0.0065 \\
8 & 1.67 & 0.80 & 0.5 & 3.65 & 0.027 & 0.0065 \\
9 & 1.67 & 0.80 & 2.0 & 5.15 & 0.027 & 0.0065 \\
10 & 1.67 & 0.80 & 2.0 & 5.15 & 0.056 & 0.0065 \\
$9 \mathrm{~T}$ & 1.67 & 0.80 & 2.0 & 5.15 & 0 & \\
\hline \hline
\end{tabular}

†Note: Acoustic tests conducted without the forward-motion duct present are denoted by an asterisk.

d diameter of equivalent circular nozzle $(\mathrm{d}=11.77 \mathrm{~cm})$

$\mathrm{d}_{1}$ diameter of nozzle at exhaust plane

$d_{o}$ diameter of plug at exhaust plane

$\ell$ length of plug from exhaust plane

$\ell_{c}$ length of constant diameter portion of plug from exhaust plane

$\sigma$ porosity (ratio of hole cross-section to plug surface area; hole diameter $=$ $0.168 \mathrm{~cm}$ )

$\mathrm{t}$ plug wall thickness 


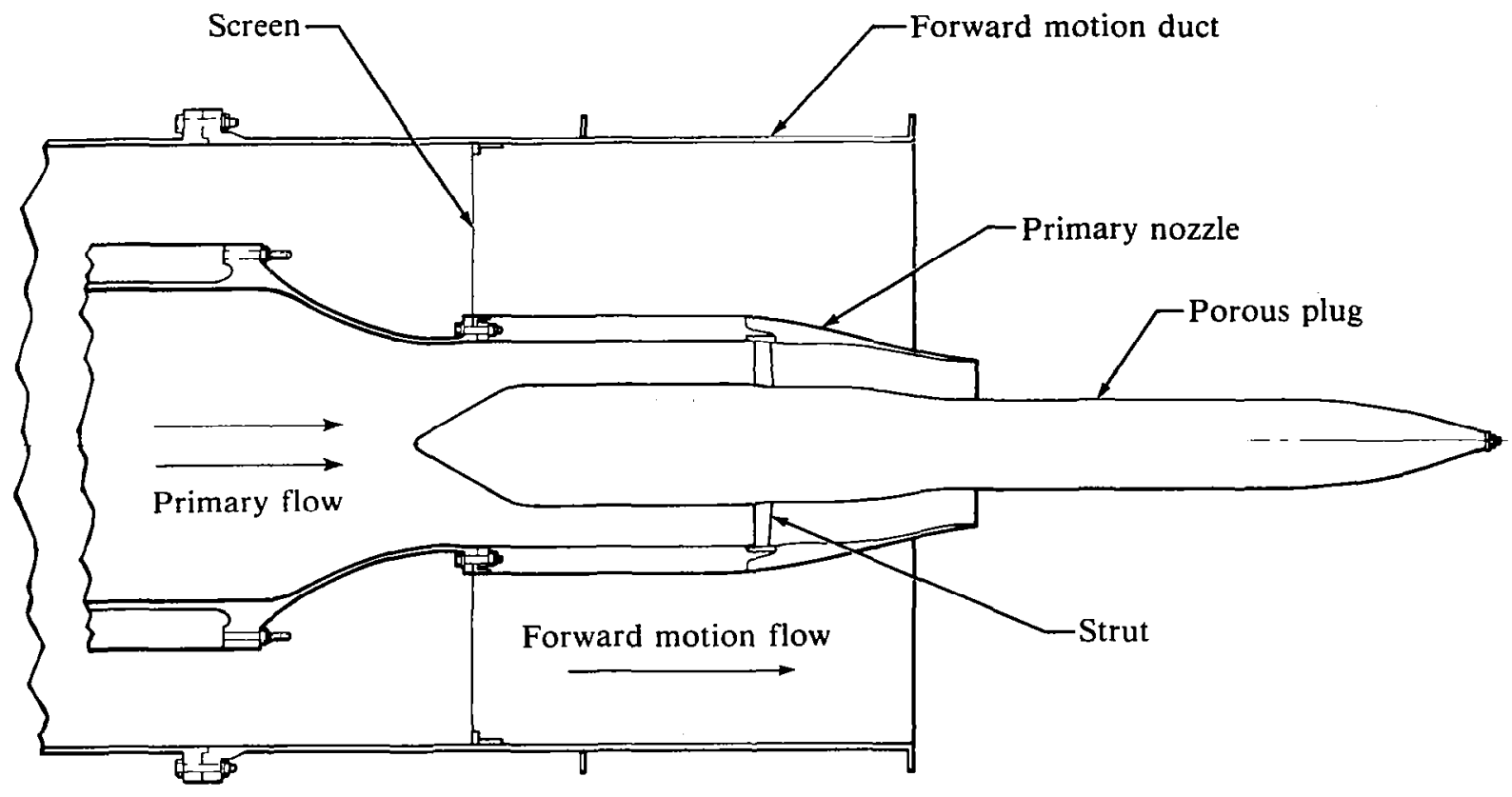

Figure 1. Flow system used for testing porous plug nozzles.

Configuration 1

Configuration 2

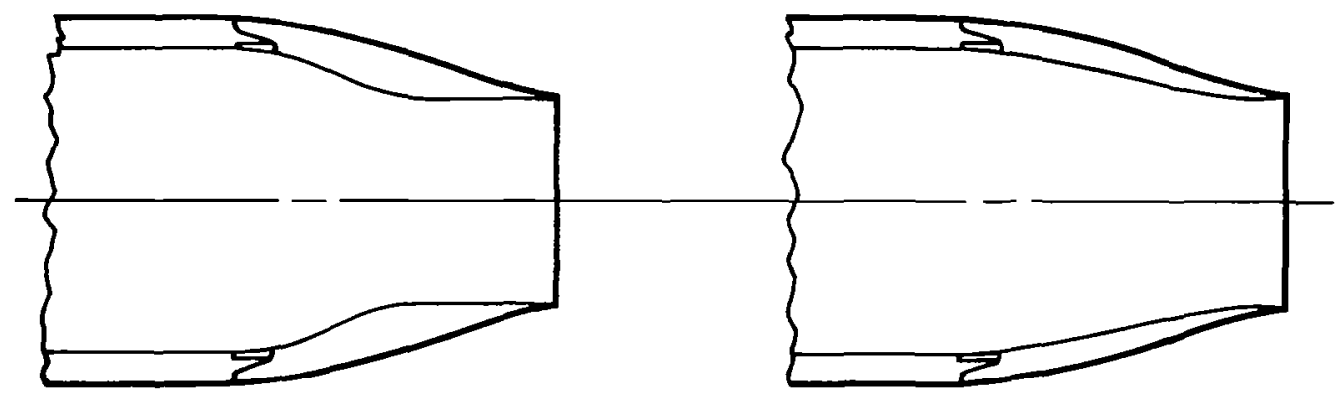

Configuration 3

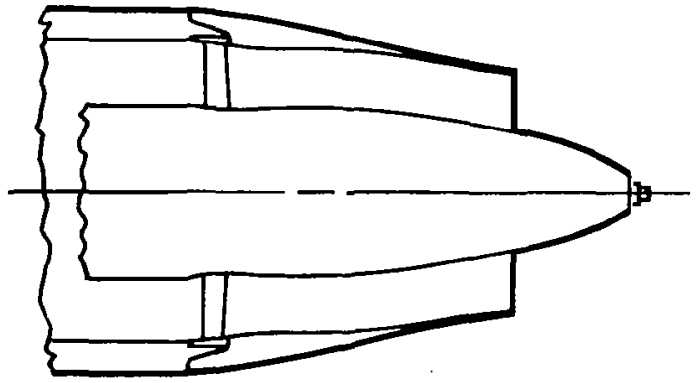

Figure 2. Sketches of model configurations 1, 2, and 3. 
Configurations 4,5 , and 6

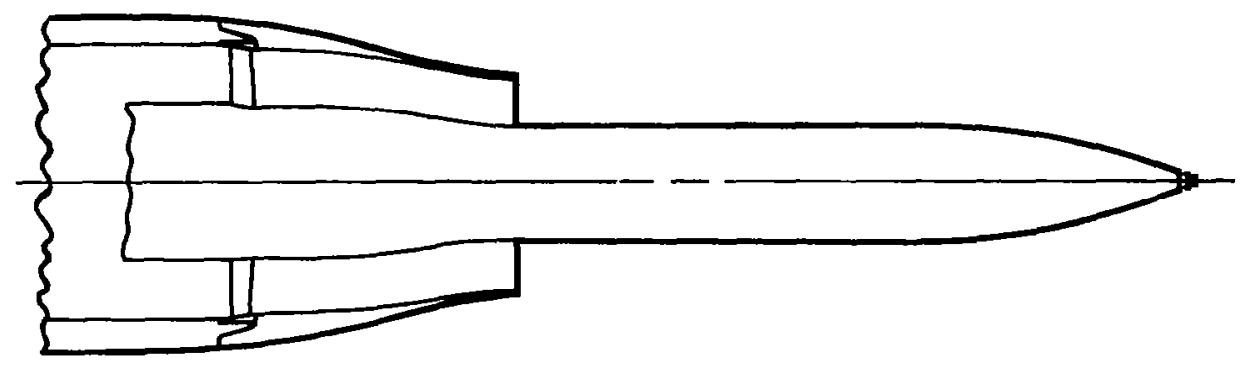

Configuration 7

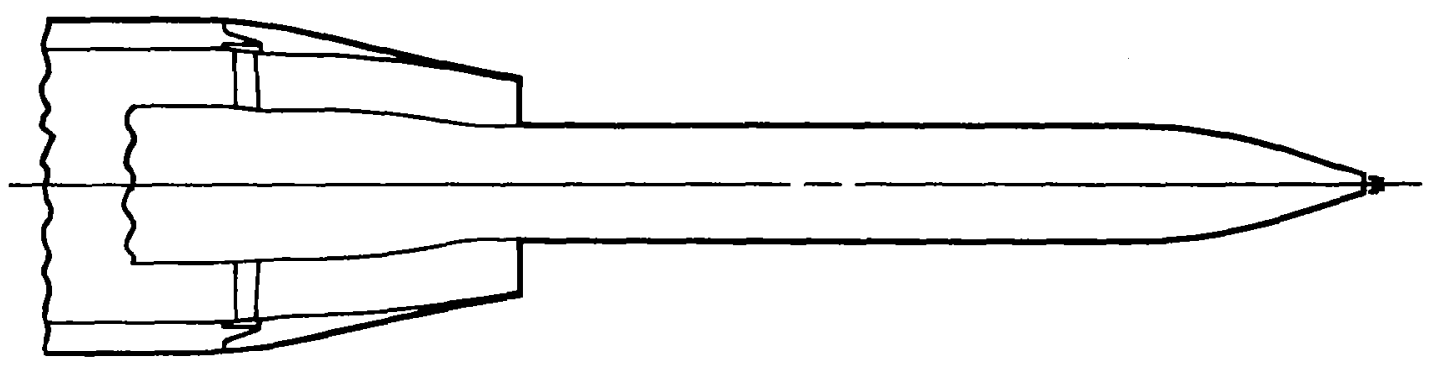

Configuration 8

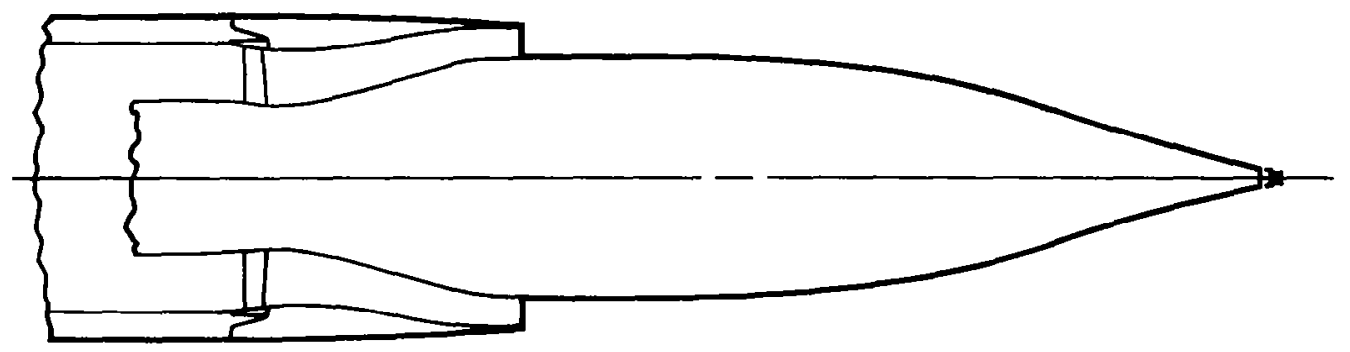

Configurations 9 and 10

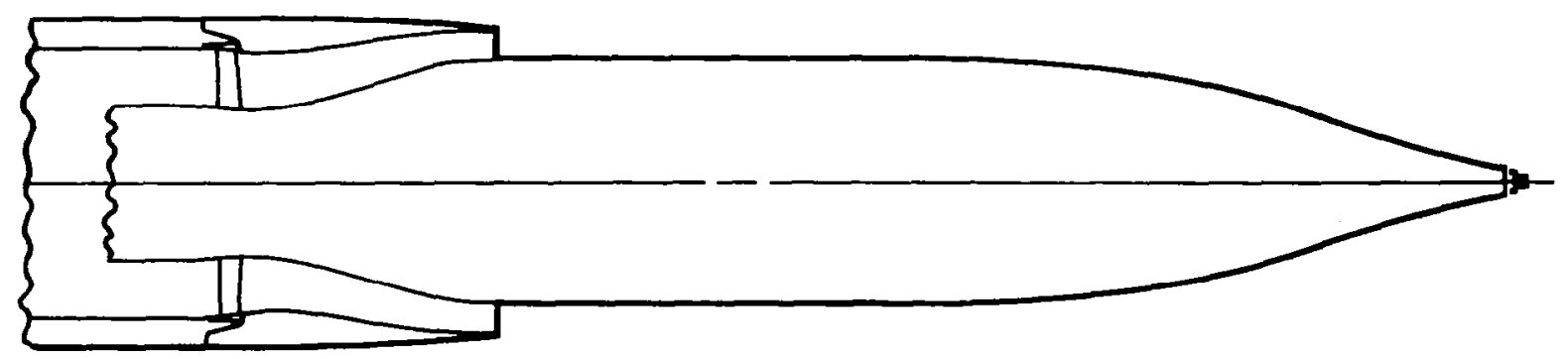

Figure 3. Sketches of model configurations 4 through 10 . 


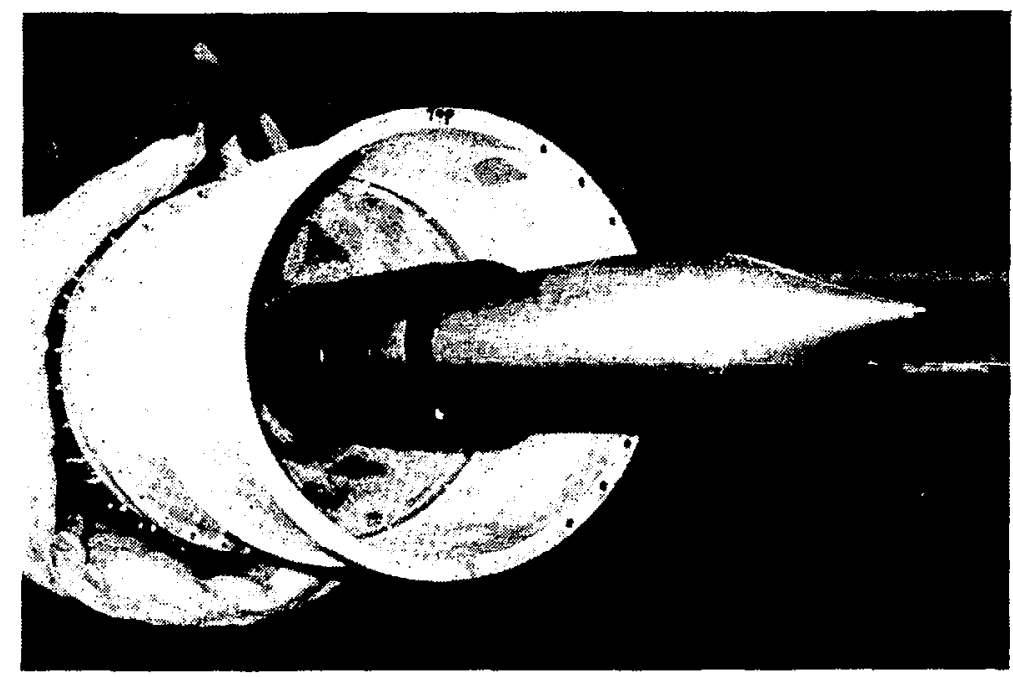

Figure 4. Model configuration 9.

radial struts; for a real engine the core turbine would be located just upstream of the struts.

Surrounding the model core is the forward motion flow system, which has a duct diameter of $50.2 \mathrm{~cm}$. A wire screen with a $50 \%$ blockage area was used to contro1 the flow unformity in the stream. The forward motion stream was operated at two speeds, either 0 or $60 \mathrm{~m} / \mathrm{s}$. The flow uniformity was checked by tufts to look for flow separations (none were found) and by a pitot tube which could be moved to any location in the forward motion exhaust plane. The measured pitot pressure was uniform within an estimated tolerance of $\pm 3 \%$, except for the boundary layers. The inner boundary layer over the nozzle was quite thin, evidently starting from near-zero thickness at the screen. The outer boundary layer was thickened by the screen support ring, resulting in a displacement thickness of about $1.0 \mathrm{~cm}$ at the exit plane.

Model Configurations 1 and 2 are reference nozzles that do not have central plugs, as shown in Figure 2. These configurations have a nozzle throat diameter of $11.77 \mathrm{~cm}$, equal to that of a circle with the same area as the throat area for Configuration 3, which has a solid plug and a nozzle like that of the CF6 engine. Hence all three configurations have approximately equal nozzle mass flows when operated at equal pressure ratios, which simplifies jet noise comparisons among the three con- 
figurations. Each of the configurations was mounted with its nozzle attached to the primary flow duct in the manner illustrated in Flgure 1. As indicated by Table 1, Configurations 4-10 have porous plugs and throat areas also corresponding to that of a $11.77 \mathrm{~cm}$ diameter circle, again for obtaining noise comparisons for a common mass flow rate for all configurations at any given pressure ratio. The models were mounted as illustrated (for Configuration 4) in Figure 1. Configuration 5 is the same as 4 except for the larger porosity, whereas 6 is the same as 4 except for the thicker skin. Configuration 7 is the same as 4 except for added length. Configurations 4-7 were designed to use the nozzle used for Configuration 3 .

Configurations 8-10 have larger plug and nozzle exhaust plane diameters than do 4, 5, 6, and 7. Configuration 10 is the same as 9 except for the porosity; Configuration 8 is the same as 9 except for the shortened length. Figure 4 is a photograph of Configuration 9.

Configuration $9 \mathrm{~T}$ is essentially the same as 9 except that the porosity was zero. Instead of manufacturing a separate plug for Configuration $9 \mathrm{~T}$, the porous plug for 9 was covered with thin $(0.063 \mathrm{~mm})$ plastic tape for use as Configuration $9 \mathrm{~T}$.

During the model checkout procedure, Configurations 1 and 4 were. operated without the forward motion duct in place. These modifications are denoted by an asterisk, as indicated in Table 1.

The nozzle and plug surface coordinates were designed to minimize any transonic flow problems that might occur. The designs were checked by using the Douglas MTRAN computer program to calculate nozzle transonic Mach number profiles across the stream and distributions along the channel walls. The program indicated that no flow problems should exist. Nozzle 1, for Configuration 1, was configured as an ASME design with a constant-diameter throat whose length is 0.65 times the diameter. Nozzle 2, for Configuration 2, was configured as a converging-diverging nozzle with a nozzle exhaust to throat area ratio of 1.03 , approximately the same as for Configuration 3. The Nozzle 2 design was such that an isomach surface for a Mach number of 1.3 was generated by the computer for the appropriate pressure ratio and located just downstream of the exhaust plane so as to show approximately uniform diverging flow. 


\section{Acoustic Testing}

The acoustic testing was done in the McDonnell Douglas Anechoic Acoustic Test Facility located in El Segundo, California. The facility is described in Reference 3.

Test Setup - Figure 5 is a plan view of the chamber showing the locations of the porous plug models and the microphones. Microphone 1 was placed just south of the jet, and microphones 2-13 were located at $10^{\circ}$ intervals measured about the chamber center, a point on the jet axis and half-way between the east and west walls. The microphone angular coordinates were chosen to match the microphone supports that had been established in the chamber.

In the earlier work of Maestrello, ${ }^{2}$ the microphone radial locations were carefully controlled so that meaningful cross-correlation measurements could be made between selected pairs of microphones. The earlier work had shown that the effect of a porous plug is to make the jet noise source region more compact than in the case of a jet without a porous plug. The microphones were centered about a point at the tip of the jet

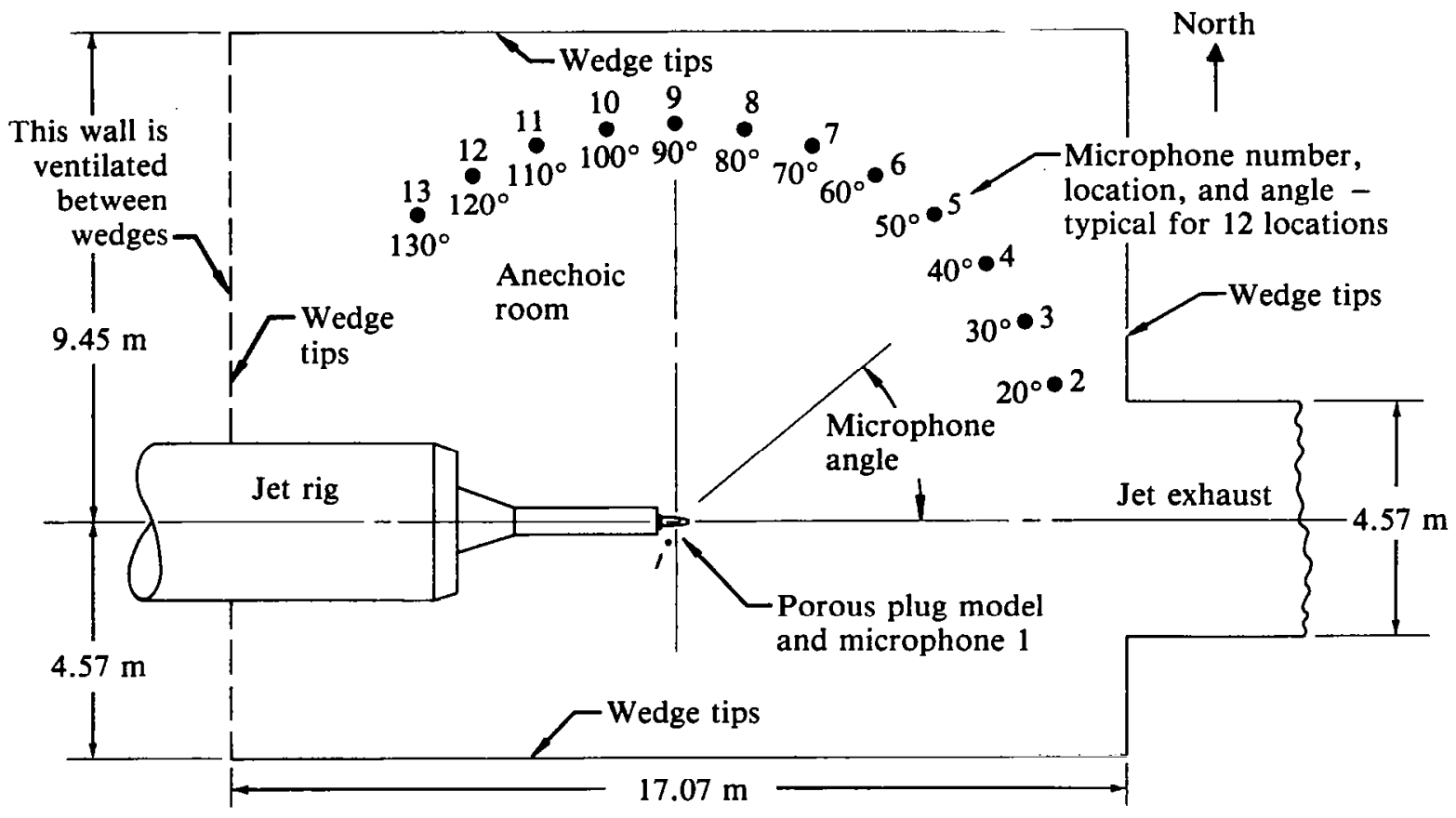

Figure 5. Plan view of anechoic room, jet rig, typical model, and microphone locations. 
potential core flow, since Maestrello expected that the notse sources would be near this point. For the new experiments, the microphones were similarly centered about the point shown in Figure 6 . The reference point was chosen with $\mathrm{X}_{\mathrm{p}}=12.7 \mathrm{~cm}(5.0 \mathrm{in.})$ and $\mathrm{Y}_{\mathrm{p}}=5.08 \mathrm{~cm}(2.00$ in.), which matches the expected location of the potential core tip for Configurations 4-7. For Configurations 8-10 the potential core tip was located approximately at $\mathrm{X}_{\mathrm{p}}=8.3 \mathrm{~cm}(3.26 \mathrm{in.})$ and $\mathrm{Y}_{\mathrm{p}}=8.8 \mathrm{~cm}(3.47$ in.), and for all configurations the chamber center was $35 \mathrm{~cm}$ ( 18.8 in.) from the nozzle exhaust plane. The microphones were $0.64 \mathrm{~cm}$ in diameter without grid caps and oriented at zero degree incidence; the microphones were moved radially to adjust the distance to the reference point as closely as practical to a distance of $762.6 \mathrm{~cm}(300.25 \mathrm{in.})$, as shown in Figure 6.

Preamplifiers were used to send the signals to a one-third octave band analyzer. The one-third octave band data were observed for all

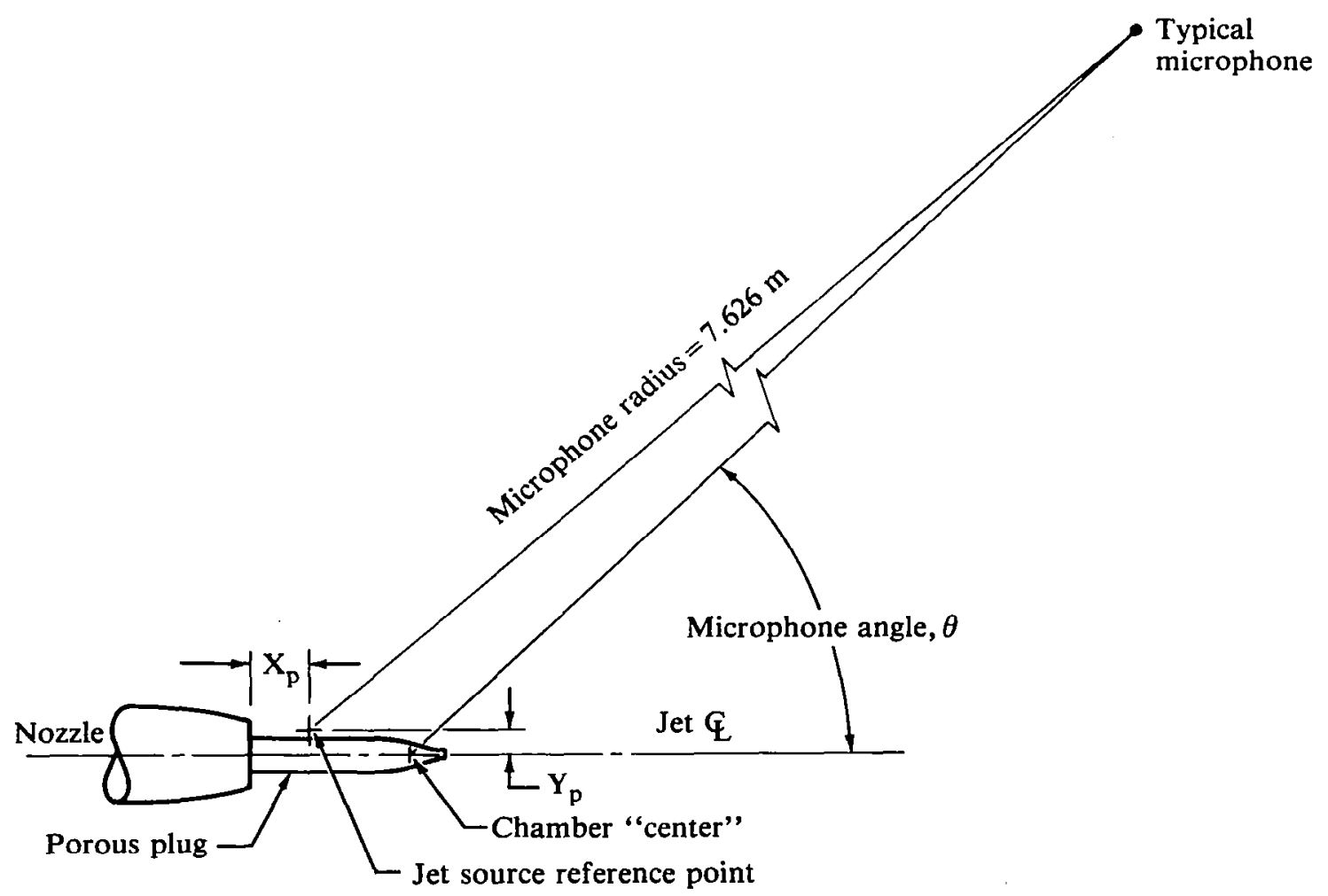

Figure 6. Microphone coordinates. 
center frequencies from $200-80000 \mathrm{~Hz}$, and the data were stored by the computer system. Twelve of the microphone signals also were recorded on analog tape with a recorder in $F M W B-I$ mode at $76.2 \mathrm{~cm} / \mathrm{s}$ for a bandwidth of $20000 \mathrm{~Hz}$.

Test Precautions - A number of preliminary tests were made to check the models and flow system for possible contamination by unwanted noise sources. The first such test was to run the jet rig and model with the primary nozzle and the plug support struts removed. The objective was to find any tones that might be generated by the flow system. The system was run at pressure ratios of 1.03 to 1.81 ; no tones were observed. Hence, the jet noise was not contaminated by upstream tones.

A few test runs were made on Configuration $1^{*}$ followed by similar runs with one temperature and two pitot tube rakes installed in the engine core duct a short distance upstream of the nozzle. Each rake consisted of five thermocouple probes or five pitot tubes. The probes and tubes were used to compare model flow conditions with the conditions upstream in the plenum chamber of the jet rig, and there was a concern that the rakes might be a significant noise source. The change in overall sound pressure levels of the jet noise as a result of inserting the rakes is shown by comparing the runs in Tables 6 and 10. The tables show that the effect of the rakes is inconclusive; only at Microphone 9 are some of the sound levels increased significantly by the rakes.

A similar test was made in Configuration $4^{*}$. In this case, the effect of adding the rakes appears to be more consistent; the rakes almost always add 0.1 to $0.2 \mathrm{~dB}$ to the overall sound pressure level (OASPL).

Because of these effects, the rakes were deleted from the remainder of the testing, with one exception: the temperature rake was installed for the test of Configuration $1^{*}$ at a total temperature of about $540^{\circ} \mathrm{C}$. The quality of the facility for jet noise research was tested by running Configuration 1 through a range of subsonic speeds to determine. the functional dependence of the noise on velocity, shown in Figure 7 . The data points, representing jet Mach numbers of 0.3 to 0.8 show that the noise at $90^{\circ}$ to the jet axis follows the Lighthill $U^{8}$-law extremely well except for a slight deviation above the $U^{8}-1$ ine at the lower 


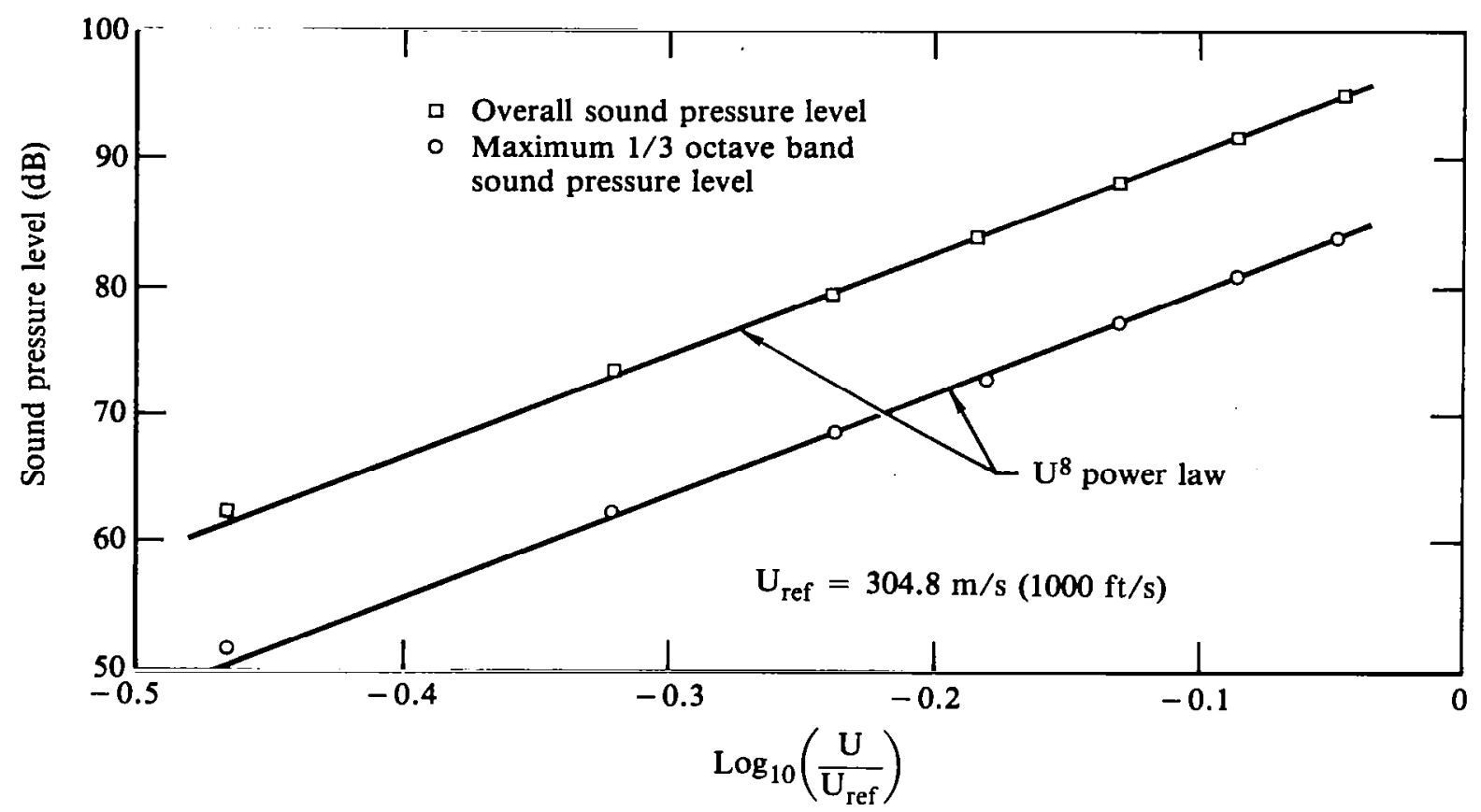

Figure 7. Jet sound pressure levels as a function of speed; model configuration 1 , microphone $90^{\circ}$ to jet axis.

speeds. The deviation may be a dipole or monopole phenomenon, as would be expected at low speeds. The data compare favorably with that of Lush, 4 who made a careful study of jet noise dependence on speed and also found a $\mathrm{U}^{8}$-dependence at $90^{\circ}$ to the jet axis. The facility and model results indicate a potential for high-quality jet noise testing.

The jet noise levels for the highest speed in Figure 7 are more than $35 \mathrm{~dB}$ above the background noise levels, reported in Reference 3 .

Test Conditions - All model configurations were tested with the primary flow total pressure ratios of $1.60,2.20,2.80,3.40$, and 4.00, with the primary total temperature at about $25^{\circ} \mathrm{C}$, and with the forward motion flow off. In addition, Configurations 1, 4, and 9 were tested with the primary total temperature at $370^{\circ} \mathrm{C}$ and again at $540^{\circ} \mathrm{C}$. Finally, Configurations 1, 4, and 9 were tested with cold primary flow $\left(25^{\circ} \mathrm{C}\right)$ and with the forward motion flow turned on. The forward motion flow had a total temperature of about $25^{\circ} \mathrm{C}$ and a jet velocity of $60 \mathrm{~m} / \mathrm{s}$. Nozzle mass flow rates calculated assuming ideal, one-dimensional flow through a nozzle throat (of area equal to $0.01088 \mathrm{~m}^{2}$ for all con- 
figurations) are given in Table 2. Also given are the Mach numbers of the jet at the nozzle exhaust plane assuming an ideal expansion of the jet flow to atmospheric pressure.

The nozzle discharge coefficients, $C_{D}$, are defined as

$$
\mathrm{C}_{\mathrm{D}}=\frac{\dot{\mathrm{m}}_{\mathrm{m}}}{\dot{\mathrm{m}}_{\mathrm{i}}} \text {, }
$$

where $\dot{\mathrm{m}}_{\mathrm{m}}$ is the measured mass flow rate and $\dot{\mathrm{m}}_{i}$ is the ideal, onedimensional mass flow rate. Values of $C_{D}$ are given in Table 3. For supersonic pressure ratios, the $C_{D}$ 's for Configurations 3-7 are essentially the same (within experimental error); hence the equal throat areas result in equal mass flow rates. The flow rates through Configuration 1 are only slightly less. The supersonic flow rates for Configurations 8-10 are about 1\% less than for 3-7 - possibly because of a more tortuous flow path between the plug and nozzle, leading to a somewhat thicker boundary-layer for Configurations 8-10. An added effect is introduced by the much larger plug and nozzle diameters on Configurations $8-10$, so that the boundary layer displacement thickness occupies more of the throat area than in the case of Configurations 3-7. Conversely, Configuration 2 has a throat that has a small diameter and is short in the streamwise direction so that the throat boundary-layer displacement thickness effect occupies little of the throat area. Configuration 1 has the same throat diameter as Configuration 2 , but the longer

TABLE 2. CALCULATED NOZZLE-MASS-FLOW RATES.

$$
\begin{gathered}
\mathrm{P}_{\mathrm{a}}=1 \mathrm{~atm}=0.1013 \mathrm{MPa} \\
\mathrm{T}_{\mathrm{o}}=25^{\circ} \mathrm{C}=296.1 \mathrm{~K}, \gamma=1.4
\end{gathered}
$$

\begin{tabular}{ccc}
\hline \hline $\begin{array}{c}\text { Pressure } \\
\text { ratio }\end{array}$ & $\begin{array}{c}\text { Ideal Mach } \\
\text { number }\end{array}$ & $\begin{array}{c}\text { Ideal mass-flow rate } \\
(\mathbf{k g} / \mathbf{s})\end{array}$ \\
\hline 1.6 & 0.8477 & 4.0444 \\
2.2 & 1.1240 & 5.6797 \\
2.8 & 1.3077 & 7.2287 \\
3.4 & 1.4467 & 8.7778 \\
4.0 & 1.5588 & 10.3268 \\
\hline
\end{tabular}


TABLE 3. NOZZLE DISCHARGE COEFFICIENTS.

\begin{tabular}{|c|c|c|c|c|c|}
\hline \multirow{2}{*}{ Configuration } & \multicolumn{5}{|c|}{ Pressure ratio } \\
\hline & 1.60 & 2.20 & 2.80 & 3.40 & 4.00 \\
\hline 1 & 0.9806 & 0.9814 & 0.9813 & 0.9862 & 0.9846 \\
\hline 2 & 1.0136 & 0.9952 & 0.9941 & 0.9986 & 0.9976 \\
\hline 3 & 1.0003 & 0.9830 & 0.9860 & 0.9867 & 0.9859 \\
\hline 4 & 0.9961 & 0.9825 & 0.9857 & 0.9862 & 0.9859 \\
\hline 5 & 0.9704 & 0.9860 & 0.9842 & 0.9855 & 0.9872 \\
\hline 6 & 0.9681 & 0.9847 & 0.9853 & 0.9849 & 0.9883 \\
\hline 7 & 0.9695 & 0.9826 & 0.9821 & 0.9872 & 0.9856 \\
\hline 8 & 0.9652 & 0.9739 & 0.9735 & 0.9729 & 0.9768 \\
\hline 9 & 0.9590 & 0.9710 & 0.9740 & 0.9767 & 0.9755 \\
\hline 10 & 0.9591 & 0.9703 & 0.9716 & 0.9729 & 0.9735 \\
\hline
\end{tabular}

throat length of Configuration 1 results in a thicker boundary layer, so that $C_{D}$ is smaller for Configuration 1 than for 2 . At the subsonic pressure ratio $(1.60)$ Configurations 8-10 again have smaller $C_{D}$ 's than Configurations 4-7, apparently for the same reasons as given for the supersonic pressure ratio cases. Configuration 3 is actually a converging-diverging type nozzle since the minimum area or throat is located a short distance upstream of the nozzle exhaust plane (in contrast to Configurations 4-10 where the throat is at the exhaust plane); hence, for the subsonic pressure ratio the throat Mach number can be greater than the 0.8477 value implied by Table 2 . Therefore, $C_{D}$ can be, and indeed is found to be greater than 1.0 . The subsonic $C_{D}$ for Configuration 2 is even larger, whereas it cannot be as large for the long, constant diameter throat of Configuration 1.

The fact that the small changes in $C_{D}$ can be related to known boundary layer and compressible flow phenomena implies that the nozzle flows were operating without any peculiar flow problems that could affect the jet noise generation. of course, the different $C_{D}$ 's indicate differing mass flow rates for the various configurations, but the differences are so small that they can be neglected. For example, an increase in the mass flow rate of $1 \%$ changes the sound power by $1 \%$ for the 
same jet velocity, which corresponds to a sound pressure change of only $0.043 \mathrm{~dB}$.

The nozzle mass flow rates varied from the values indicated by Tables 2 and 3 because of small changes in the anecholc chamber atmospheric pressure, small errors in setting the nozzle pressure ratios, and small variations in the air supply total-temperature. The most serious of these changes was the varlation in supply temperature for the cold runs, inasmuch as the anechoic chamber has no provision for heating or cooling the air by small, controllable amounts. The supply-air temperature varied from day to day and run to run because of changes in the large, outdoor supply tank temperature and because of frictional heating in the supply pipes and in the jet rig. Table 4 shows the actual total temperature variations during the cold flow testing of Configurations 110. Fortunately, the nozzle mass flow rate varies as the inverse square root of the total temperature, so that the mass flow varies more slowly than the temperature, and the temperature variations of Table 4 correspond to a mass flow variation of $\pm 1.0 \%$. The corresponding variation in jet noise sound pressure level has been estimated by comparing hot $\left(370^{\circ} \mathrm{C}\right)$ and $\operatorname{cold}\left(25^{\circ} \mathrm{C}\right)$ run data taken with Configurations 1 and 4. Interpolation of the results shows that a $5^{\circ} \mathrm{C}$ change in temperature can

TABLE 4. NOZZLE AIR SUPPLY TOTAL-TEMPERATURE VARIATION IN THE ANECHOIC CHAMBER.

\begin{tabular}{cc}
\hline \hline Configuration & $\begin{array}{c}\text { Total-temperature range } \\
\text { (K) }\end{array}$ \\
\hline 1 & 296.0 to 299.1 \\
2 & 292.6 to 297.8 \\
3 & 289.9 to 294.4 \\
4 & 289.6 to 295.2 \\
5 & 293.3 to 293.8 \\
6 & 297.0 to 299.8 \\
7 & 299.9 to 301.4 \\
8 & 297.7 to 298.6 \\
9 & 293.3 to 301.1 \\
10 & 292.3 to 294.0 \\
\hline \hline
\end{tabular}


change overall sound pressure levels between 0 and $0.17 \mathrm{~dB}$ for a microphone at $60^{\circ}$ from the jet axis. At $90^{\circ}$ from the jet axis the change is up to $0.13 \mathrm{~dB}$. These sound pressure level changes occurred at a pressure ratio of 1.60 . For the higher pressure ratios, the sound level changes are smaller. These changes were considered to be so small that they could be accepted as a part of the system experimental error.

During all tests, the jet rig was used to measure the total thrust of the primary and the forward-motion jets, and a thrust coefficient was computed - the ratio of the measured thrust to the ideal thrust; that is, the thrust coefficient $C_{T}$ is given by:

$$
\mathrm{C}_{\mathrm{T}}=\frac{\mathrm{T}_{\mathrm{m}}}{\sum_{j=1}^{2} \dot{\mathrm{m}}_{\mathrm{mj}} \mathrm{U}_{i j}},
$$

where $j$ is the flow index ( 1 for the primary flow and 2 for the forward motion flow), $T_{m}$ is the measured thrust, $\dot{\mathrm{m}}_{\mathrm{m}}$ is the measured mass flow rate, and $U_{i j}$ is the calculated ideal jet velocity.

\section{Aerodynamic Testing}

The aerodynamic testing of the porous plug nozzles was planned and performed at the McDonnell Douglas Research Laboratories in St. Louls, Missouri. The aerodynamic testing was conducted after completion of the acoustic testing, and the acoustic results were used in planning the aerodynamic test program. The tests were motivated by the need to establish the relationship between the detailed flow characteristics and the propagating acoustic field. The control and suppression of jet noise by an extended porous centerbody is closely linked to the interaction between the plug and the surrounding annular jet. A characterization of the noise suppression mechanisms is needed to optimize such devices for noise reduction with minimal fuel consumption penalties.

Based on acoustic measurements and shadowgraph flow visualization, Maestre110 ${ }^{2}$ hypothesized that the primary noise reduction effect of porous plug nozzles at supersonic velocities is the elimination of shock-associated noise. The shadowgraph visualization indicated a gen- 
erally shock-free core flow, and he proposed two reasons for the decrease in shock intensity:

1. The surface porosity acts to equalize the static pressure across impinging shocks and expansion waves, thereby reducing the strength of their reflections.

2. The flow geometry is modified by the presence of the plug from an axisymmetric circular jet to the annular equivalent. As the plug-to-nozzle diameter-ratio is increased, the annular flow becomes more clearly two-dimensional, and the compression wave intensification (or focusing) that occurs at the center of a circular jet is suppressed.

The results obtained by Maestrello provided a starting point for the current study. Several experimental techniques were used to investigate the apparent elimination of shock cells. The streamwise and radial components of velocity were measured using a two-color laser Doppler velocimeter system (LDV), to evaluate mean velocities, turbulence intensities, and the turbulent Reynolds stress. Flow visualization was performed using spark shadowgraphs for instantaneous pictures and high-speed shadowgraph movies to observe the unsteady aspects of the jet flowfield. Plug surface and freestream static pressures were measured using static pressure ports and a static pressure probe, respectively, and two near-field microphones were employed for additional sound pressure level measurements. The experimental configuration is shown schematically in Figure 8.

Test Setup - The aerodynamic tests were conducted in the McDonne11 Aircraft Company (MCAIR) Free Jet Test Facility, a part of the Propulsion Subsystem Test Facility in St. Louis. It consists of a diffuser and settling chamber which terminate in a universal adaptor flange for attachment of test articles. During tests, the $4 \times 10 \mathrm{~m}$ test cell is opened to an acoustically treated exhaust shed. Constant mass flow rates up to $18 \mathrm{~kg} / \mathrm{s}$ can be maintained through a digital computer-controlled valve system. Specific test conditions are established by metering the high-pressure air avallable from the MCAIR Polysonic Wind Tunnel air storage system. Operational parameters and test data are re- 


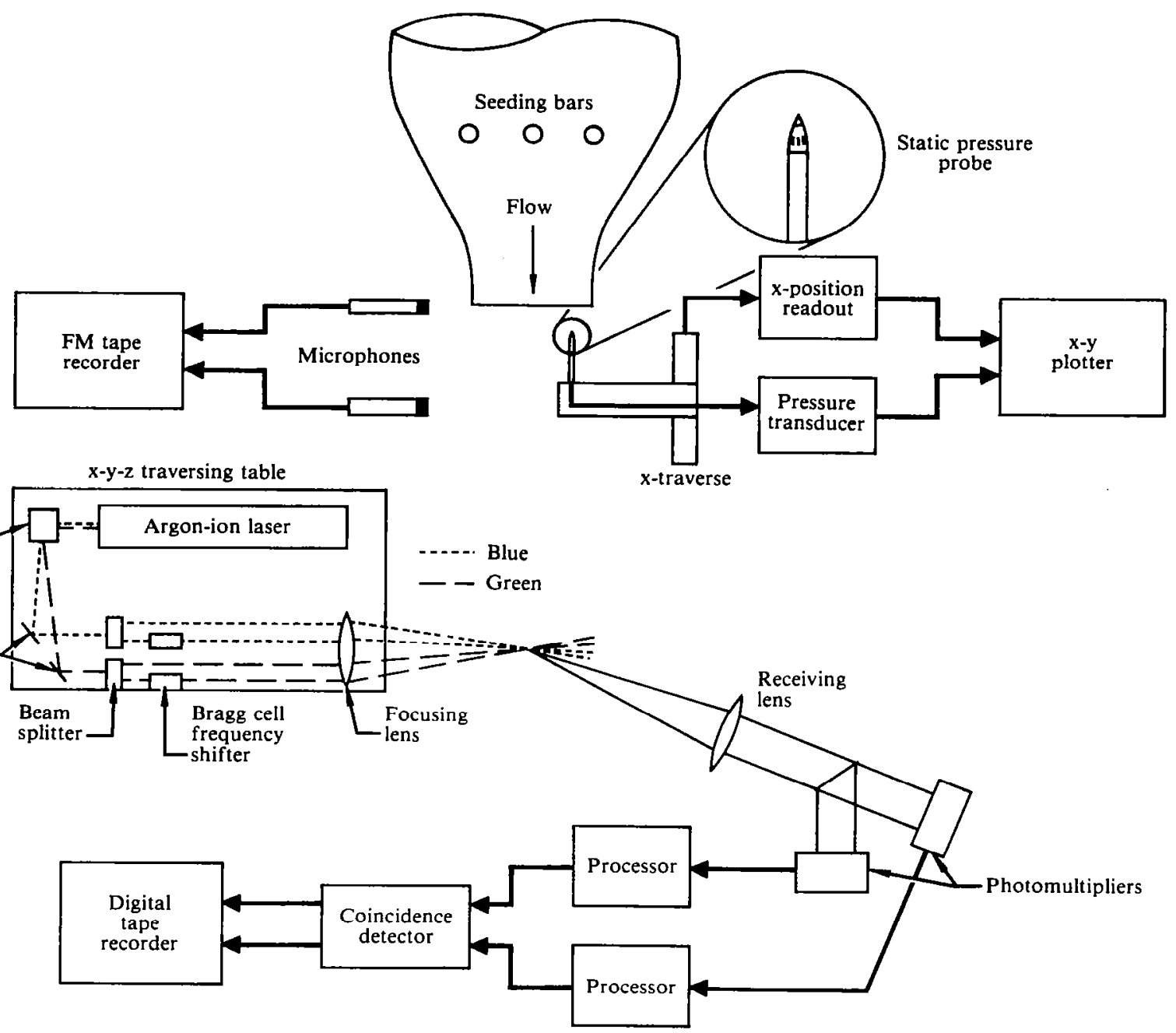

Figure 8. Flow measurement instrumentation.

corded in the adjacent control room using an on-site digital data acquisition system.

Because of the complexity of the aerodynamic tests, a subset consisting of five nozzle configurations was chosen for this portion of the investigation (Table 5). The particular configurations were chosen to characterize the trends observed in the acoustic test results. Configuration 2, which is a Mach 1.3 reference nozzle, produces a well-defined shock cell structure when operated at other than design conditions. It provided reference data on shock trains in a free jet, and the distinct shock structure was useful for evaluating the performance of the static 
TABLE 5. NOZZLE CONFIGURATIONS USED FOR AERODYNAMIC TESTS.

\begin{tabular}{c}
\hline Configuration $\left(\sigma, \mathrm{d}_{0} / \mathrm{d}_{1}, \ell / \mathrm{d}\right)$ \\
\hline $2(0,0,0)$ \\
$6(0.03,0.53,3.4)$ \\
$8(0.03,0.80,3.7)$ \\
$9 \mathrm{~T}(0,0.80,5.2)$ \\
$10(0.06,0.80,5.2)$ \\
\hline
\end{tabular}

pressure probe. Configurations 6 and 10 were chosen because they produce the greatest sound suppression of the 0.53 and 0.80 plug-todiameter ratio nozzles, respectively. Configuration $9 \mathrm{~T}$, which is geometrically identical to Configuration 10, was reconfigured to zero porosity for the aerodynamic tests by covering the surface of the Configuration 9 nozzle with tape. The solid surface plug configuration was not tested in the acoustic facility; it was used in the aerodynamic tests as a zero-porosity reference configuration geometrically similar to the porous plugs. Configuration 8 , which also produced significant sound reduction, was chosen because it has a surface porosity intermediate between Configuration $9 \mathrm{~T}$ and 10 , and because it offers a direct comparison with Configuration 6 in measuring the effect of plug-tonozzle diameter ratio. The notation described in Table 5 outlines the pertinent geometrical properties of the nozzles chosen for the aerodynamic tests, and facilitates comparisons among the various geometries. The aerodynamic tests were conducted for five nozzle pressure ratios $(1.6,2.2,2.8,3.4$, and 4.0$)$ to maintain consistency with the acoustic tests. A conical contraction was constructed to mate the nozzles to the adaptor flange of the Free Jet Facility, and the contraction was faired into the nozzles used for the acoustic tests. No provisions were made for jet heating or forward flight simulation, and the aerodynamic tests were limited to cold jets exhausting into ambient air. 
Laser Doppler Velocimeter Systen - The high velocities encountered In the core flow of a supersonic jet impose severe restrictions on velocity measurement techniques. Conventional hot-wire measurements are difficult in such an environment. A two-component LDV system increases the feasibility of such measurements, although the conditions in a highspeed turbulent jet are difficult even for this technique.

The two-component LDV system used for the velocity measurements is shown schematically in Figure 8. The light source is a $4 \mathrm{~W}$ argon-ion laser that produces a multi-color beam with maximum power at wavelengths of $514.5 \mathrm{~nm}$ (green) and $488.0 \mathrm{~nm}$ (blue). The LDV was operated in a twocolor, dual-beam, off-axis forward-scatter mode. The laser beam is collimated and passed through a color-splitting prism, which separates the colors into two distinct beams. Each color is processed in a similar fashion; beam splitters separate the incident beam into two beams that are focused approximately $300 \mathrm{~mm}$ beyond a focusing lens. Such an optical arrangement produces a sensing volume with a streamwise resolution of $0.5 \mathrm{~mm}$ and a fringe spacing of $17 \mu \mathrm{m}$, so that a seed particle traveling at $100 \mathrm{~m} / \mathrm{s}$ produces a Doppler frequency of approximately 8.5 MHz. To detect reversed flow (particularly in the radial direction), a Bragg cell is incorporated in the optical path of one of the beams to shift the color by $40 \mathrm{MHz}$. The shift effectively produces moving fringes in the sampling volume, so that a stationary particle will produce a Doppler frequency of $40 \mathrm{MHz}$. The receiving optics are oriented $20^{\circ}$ from the optical axis to limit the sampling volume length to $3.5 \mathrm{~mm}$ along the optical axis. The operating LDV system is shown in Figure 9.

The optics are oriented so that the sensing volumes for both colors are spatially coincident and the fringe patterns are normal to each other; thus, two velocity components can be measured simultaneously at a single point in space. Because of the large difference in the magnitudes of the streamwise and radial velocity components, each of the fringe patterns is oriented at $45^{\circ}$ to the streamwise direction. If the fringe patterns were oriented parallel and normal to the streamwise direction, a particle could pass through the sensing volume before crossing enough fringes to produce a detectable radial velocity. The $45^{\circ}$ orientation eliminates this problem by effectively detecting $(U+u)+$ 


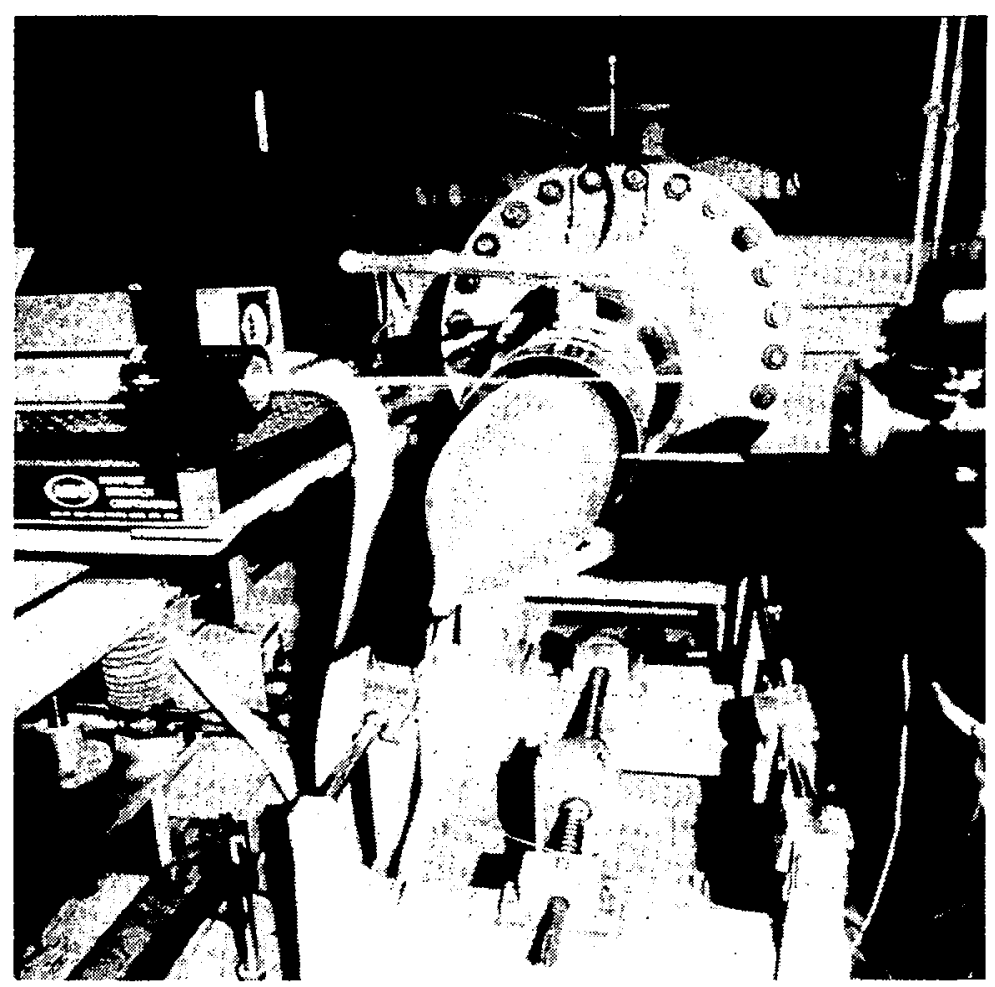

Figure 9. Porous plug nozzle aerodynamic tests.

$(\mathrm{V}+\mathrm{v})$ and $(\mathrm{U}+\mathrm{u})-(\mathrm{V}+\mathrm{v})$. The streamwise and radial velocity components are then recovered by forming the sum and difference of the measured components.

The light scattered by seed particles passing through the sensing volume is collected by the receiving optics, split according to color, and directed to photomultiplier tubes. The output signals from the photomultipliers are processed by counter-type LDV processors which employ adjustable threshold and overload settings with a Schmitt trigger and 10:16 comparator logic to ensure the processing of only Doppler bursts from individual small particles. The digital output of each processor is further qualified through a coincidence detector, which ensures that the same particle has been detected by each channel. Data from both channels are then transferred to digital magnetic tape for later offline processing.

The laser and transmitting optics are mounted on an optical table that can be traversed in three directions. The receiving optics are attached to the table through a truss support so that the entire forward 
scatter system can be traversed as a unit. The table is driven by three stepper motors, and the absolute position is determined through precision linear potentiometers. The position information is also recorded on magnetic tape, so that spatial coordinates are available to the velocity processing program.

Seeding of the jet and entrained external air was accomplished by injecting atomized dioctyl phthalate, with a mean droplet diameter of $0.8 \mu \mathrm{m}$. Seeder bars were installed in the plenum chamber upstream of the nozzle, and the seeded air was subsequently accelerated through the nozzle to ensure that the particles followed the mean flow. Particles were introduced into the ambient room air above the nozzle through an additional rake of seeding bars to provide adequate seeding of the entrained air; the bars are visible above the plug nozzle in Figure 9. Care was exercised in adjusting the relative seed densities from each of the sources because measurements in the jet shear layer could be biased by unequal seed concentrations in the plenum and entrained air.

Despite the care exercised in performing the LDV measurements, several problems limited the scope of the results. Because of the large difference between the streamwise and radial components of velocity, small rotational misalignments in the two-color system resulted in large relative errors in the measured radial velocity. It is estimated that the system can be aligned to an absolute accuracy of approximately $1^{\circ}$, and the alignment error can bias the measured radial velocity by as much as $7 \mathrm{~m} / \mathrm{s}$ for a streamwise velocity of $400 \mathrm{~m} / \mathrm{s}$. The radial velocity bias is of the same order of magnitude as the measured radial velocity, so that measurements can indicate the radial velocity only within an additive constant which varies as the optical alignment is readjusted. Since the alignment was not changed within a single profile, trends that occur within a profile can be meaningfully evaluated.

Another problem resulted from the inability to adequately seed the flow in the vicinity of the plug surface. Installation of seeding bars inside the plug was not practical, and particles were not able to follow the flow through the surface pores. The large difference between the streamwise and radial velocity components led to shallow particle trajectories, and the particles near the plug tended to impact the sur- 
face. The resulting extremely low data rates prevented unbiased velocity estimates in the vicinity of the plug surface.

The third problem, which severely degraded velocity measurements in the jet shear layer, was the formation of fog resulting from the mixing of cold jet air and nearly saturated amblent air. Because of scheduling limitations, the aerodynamic tests were conducted at night when a high humidity condition existed outside the building. The large entrainment rates in the jet reduced the pressure in the test cell below atmospher$i c$, and moist air was ultimately drawn into the room. The water droplets, which formed as a consequence of mixing, were larger and more abundant than the seed particles. They tended to lag behind the flow and produce high data rates. The velocity probability density curves shown in Figure 10 demonstrate the errors which occur as a result of the fog. When no seeding is used, the probability density function has a single peak at approximately $180 \mathrm{~m} / \mathrm{s}$. When the seed generators are

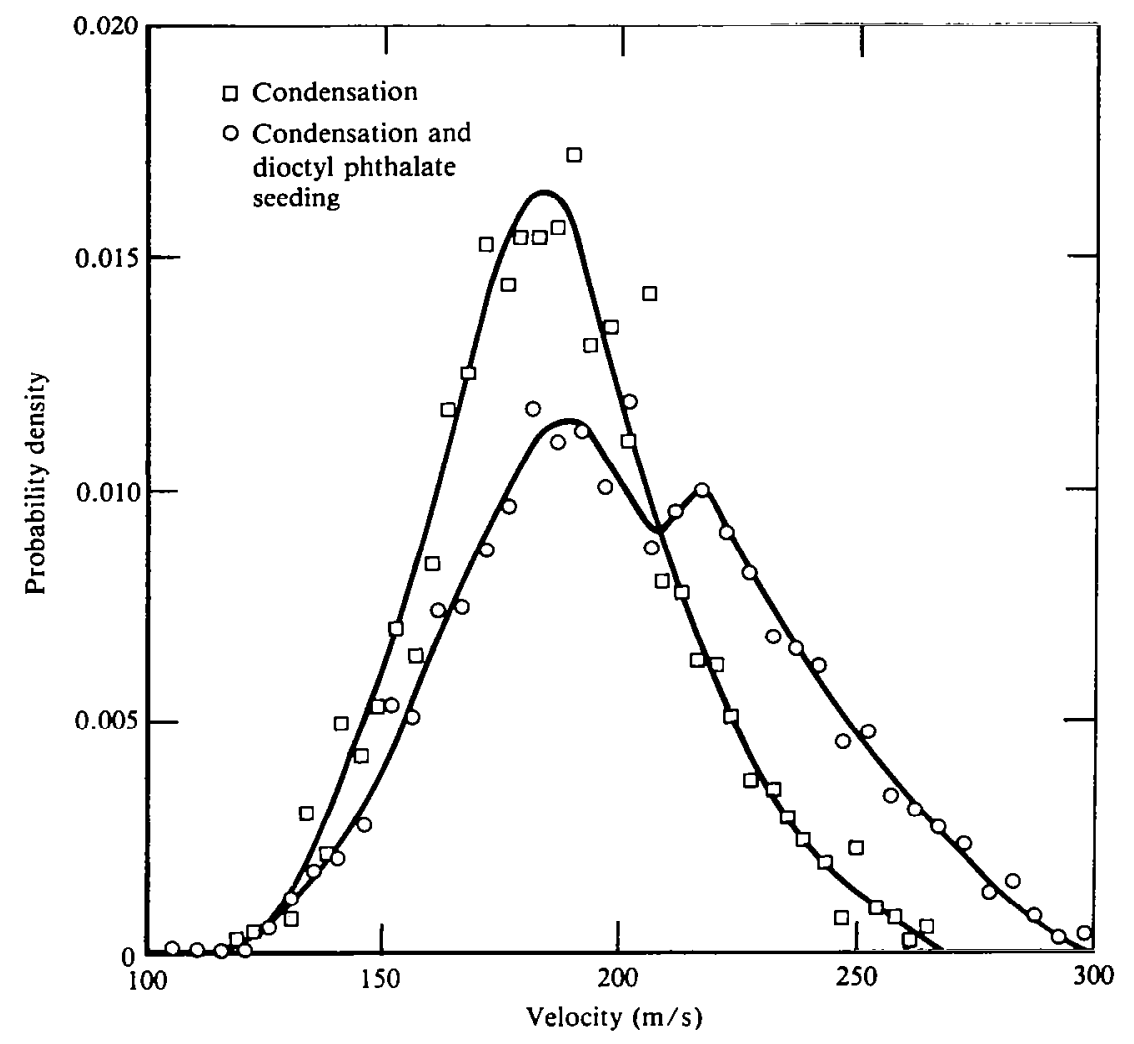

Figure 10. Effect of water condensation in shear layer on measured velocity histograms. 
turned on, the function exhibits a bimodal behavior; the original peak caused by the fog droplets is reduced, and a second peak corresponding to the true flow velocity occurs at $230 \mathrm{~m} / \mathrm{s}$. Only when the original peak is eliminated will the velocity estimate be accurate, and any significant fogging reduces the measured apparent velocity.

Several steps were taken to reduce the velocity bias due to fog formation. The compressed air supplied to the jet plenum was heated to approximately $10^{\circ} \mathrm{C}$ above ambient, although the rapid expansion at the jet exit still produced temperatures below ambient. Air entry into the test cell was minimized with plastic film, and recirculation of jet exhaust air was maximized. Thus, the fog formation was significantly reduced, although velocity measurements could not be made at pressure ratios above 3.4 and measurements in the shear layer at pressure ratios above 2.2 remained biased. Consequently, the majority of the LDV measurements were concentrated in the core flow of the jet, and velocity profiles were obtained in the streamwise direction at a radius midway between the plug surface and nozzle lip.

Static Pressure Measurements - Such limitations, coupled with the time consuming nature of the LDV measurements, reduced the usefulness of the LDV in characterizing the structure of the plug nozzle flowfield. To supplement the velocity data, detailed static pressure measurements were obtained throughout the core flow to develop a global picture of the flowfield characteristics. A static pressure probe of double conic shape (Figure 8), similar to that developed by Pinckney, ${ }^{5}$ was used for this portion of the investigation; a similar probe has been successfully used by Seiner and Norum ${ }^{6}$ to characterize the structure of underexpanded jet plumes. Since idealizing assumptions are required in interpreting the data acquired with such a probe, several diagnostic measurements were performed to verify the rellability of the results (they are discussed in the aerodynamic test results).

The probe was supported in the jet by a sting that could be traversed in both streamwise and radial directions. A pressure transducer was used to convert the probe output to an analog voltage, and a linear potentiometer provided a readout of the streamwise probe position. The output of the device was recorded on an $x-y$ plotter, and a series of 
streamwise pressure profiles was obtained at various radial posttions. The individual profiles were subsequently digitized and smoothed using a cubic-spline curve-fitting routine, and converted into a two-dimensional representation of the pressure field using various digital techniques. The smoothing and plotting of pressure data, as well as some of the processing of the velocity data, were performed on a PDP 11/70 minicomputer system at the MDRL Flight Sciences Department in St. Louis. Additional data processing techniques used to enhance various features of the pressure field are also discussed in the aerodynamic test results.

In addition to the flowfield static pressure measurements, the Configuration 8 porous plug was instrumented with static pressure ports to determine the surface pressure distribution. The ports were installed in several of the plug surface holes (see Figure 11) and were individually connected to a pressure transducer through a multiple-port valve. Mean pressure measurements were recorded for several flow conditions using the test facility data logging system.

Microphone Measurements - Two near-field microphones were included in the aerodynamic test instrumentation to make comparisons with the acoustic test results. One microphone was located in the nozzle exit plane, $30 \mathrm{~cm}$ from the nozzle $11 \mathrm{p}$, and the second at the same radial distance, $30 \mathrm{~cm}$ downstream from the exit plane. The output signals were recorded on analog FM tape for later analysis, and standard pistonphone data were recorded for calibration purposes. The acoustic data were later analyzed using an rms meter to obtain overall sound pressure levels and a spectrum analyzer to obtain narrowband spectra.

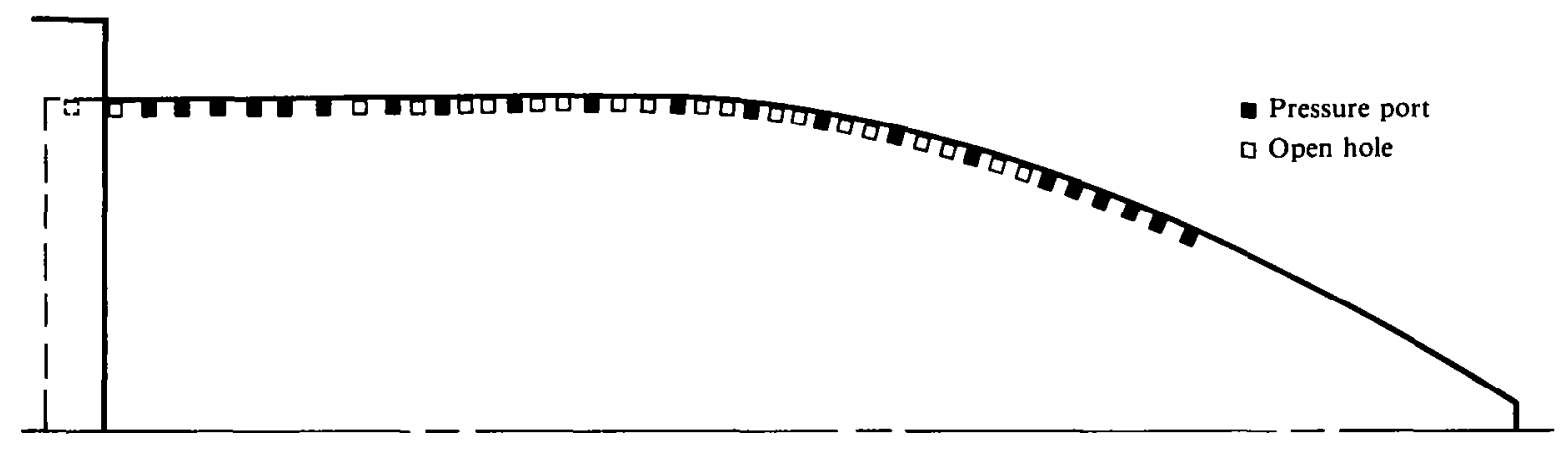

Figure 11. Pressure port locations for Configuration $8(0.03,0.80,3.7)$. 
Flow Visualization - Flow visualization was performed at pressure ratios above 1.6 using the shadowgraph technique. Instantaneous pictures were obtained through the use of a spark light source and a $35 \mathrm{~mm}$ still camera. Results were obtained for all configurations and pressure ratios, and examples of each case are presented in Appendix B. Highspeed shadowgraph movies were obtained for each configuration at a pressure ratio of 2.8 using a continuous 1 ight source and a camera operating at 5000 frames per second. The purpose of the movies was to determine the degree of unsteadiness in any shock cell motion and investigate the possibility of interaction between the shear layer and the shock cell structure. 
TEST RESULTS

Both the acoustic and aerodynamic test results are given below.

\section{Acoustic Test Results}

The acoustic results were found to agree generally with the findings of Maestre11o, 2 even though the present porous plug geometries were somewhat different and the model scale was larger than that used by Maestrello. In addition, the effects of forward motion flow, hot primary flow, and a wider range of porous plug geometric parameters are shown.

Cross-Correlation Measurements - A sample of the normalized crosscorrelation results taken with respect to the $30^{\circ}$ microphone is given by Figure 12, which shows that the correlation maximums are much closer for the porous plug suppressor (Configuration 9) than for the reference nozzle (Configuration 2). Therefore, the suppressor sound sources appear to be concentrated within a more limited region of the shear layer than for the reference nozzle.
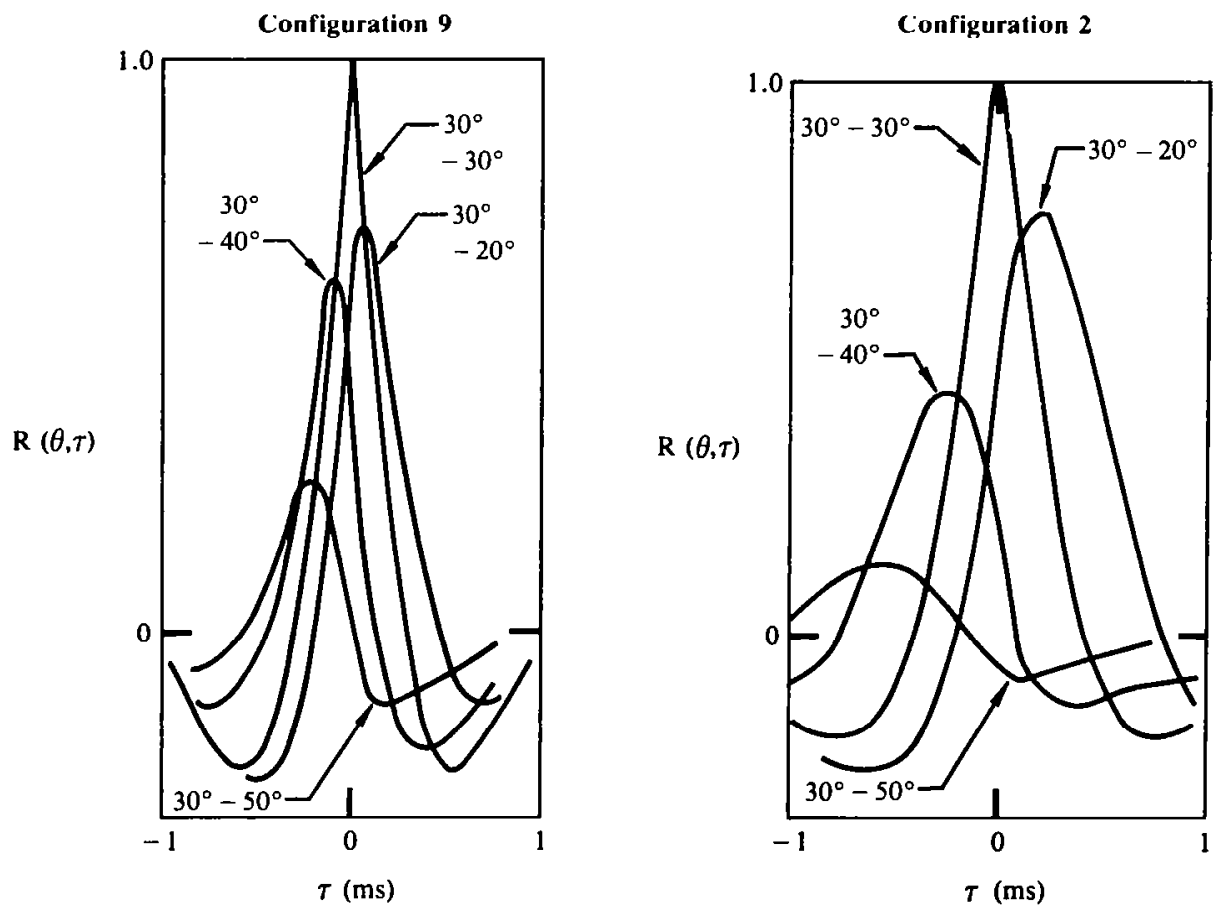

Figure 12. Normalized cross-correlation results for configurations 2 and 9 for various angular separations at a nozzle pressure ratio of 1.60 . 
At $90^{\circ}$ from the jet axis, the jet spectra contain less lowfrequency noise, and the cross-correlation plots are narrower than at $30^{\circ}$, as shown by Maestrel10.2 Figure 13 shows normalized crosscorrelation plots at $90^{\circ}$ from the jet axis for Configurations 10 and 1 . Again, for the porous plug suppressor (Configuration 10) the correlation maxima are closer than for the reference nozzle (Configuration 1). Similar results were obtained for the other porous plug and reference nozzle configurations. The porous plugs with the larger radius ratios (Configurations 8-10) had narrower correlation curves (1.e., spectra weighted by less low frequency noise) than those with the smaller radius ratios (Configurations 4-7), as illustrated in Figure 14, but these differences were not as large as the difference between the smaller radius ratio plugs and the reference nozzles (Configuration 1 and 2). The effect of heating the primary flow was to make the correlation plots narrower, as shown in Figure 15 and by comparing Figures 16 and 13.
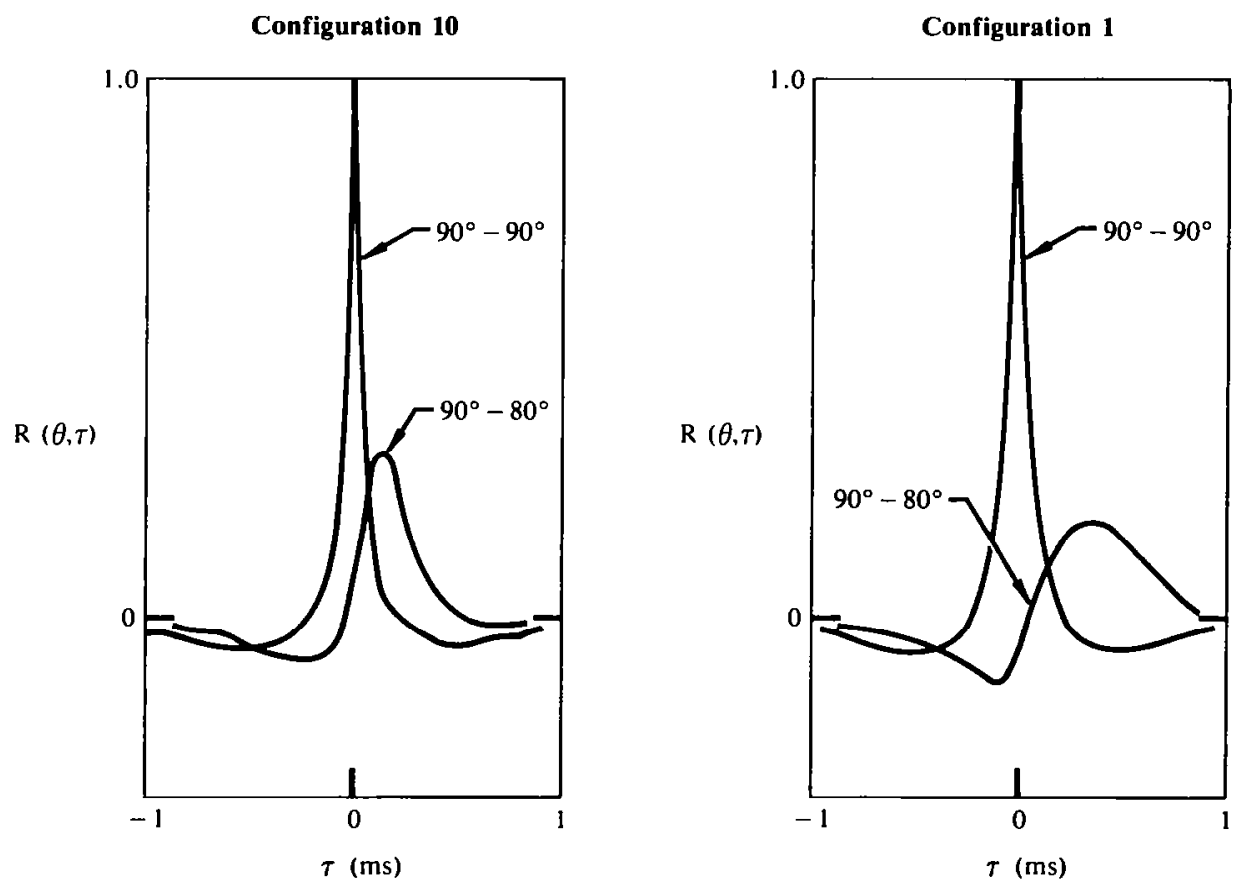

Figure 13. Normalized cross-correlation results for configurations 1 and 10 for various angular separations at a nozzle pressure ratio of 1.60 . 

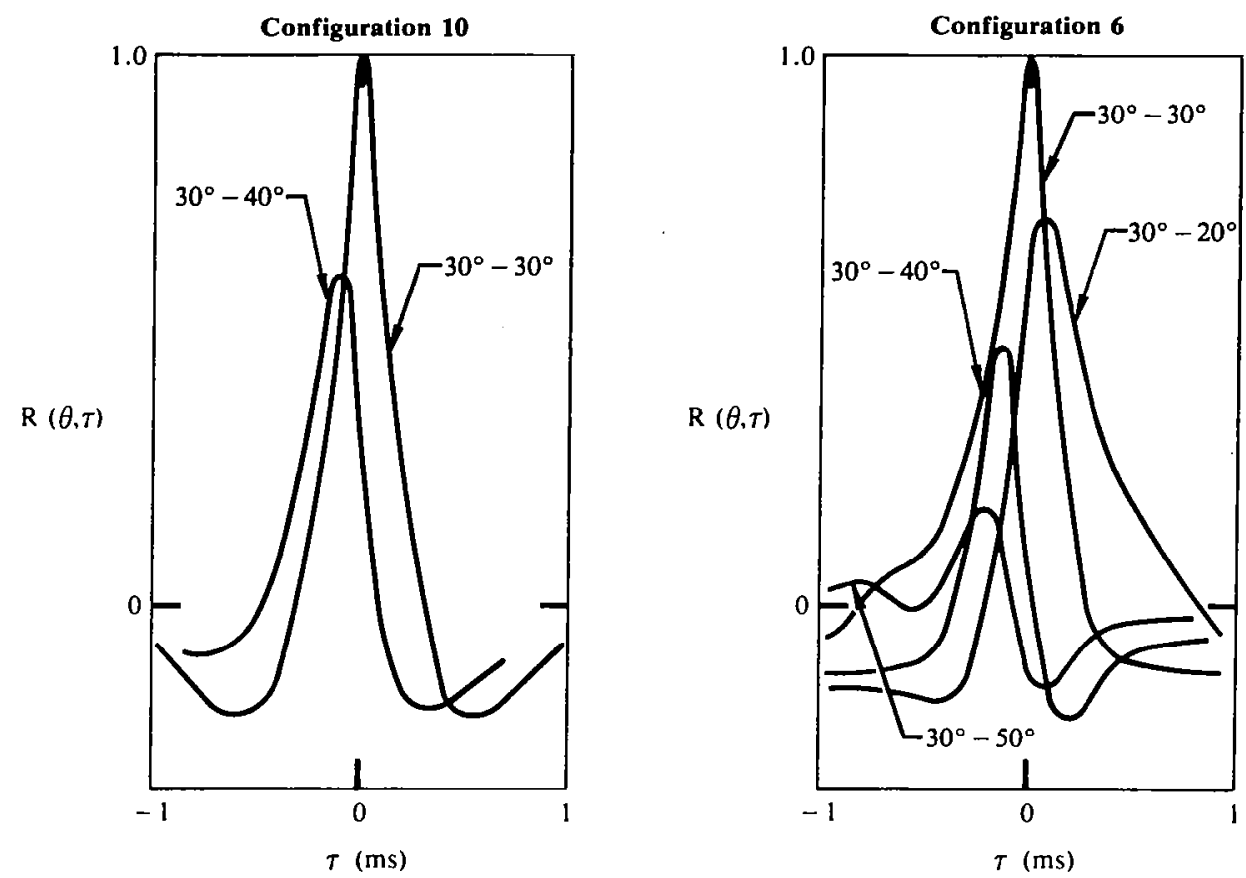

Figure 14. Normalized cross-correlation results for configurations 6 and 10 for various angular separations at a nozzle pressure ratio of 1.60 .
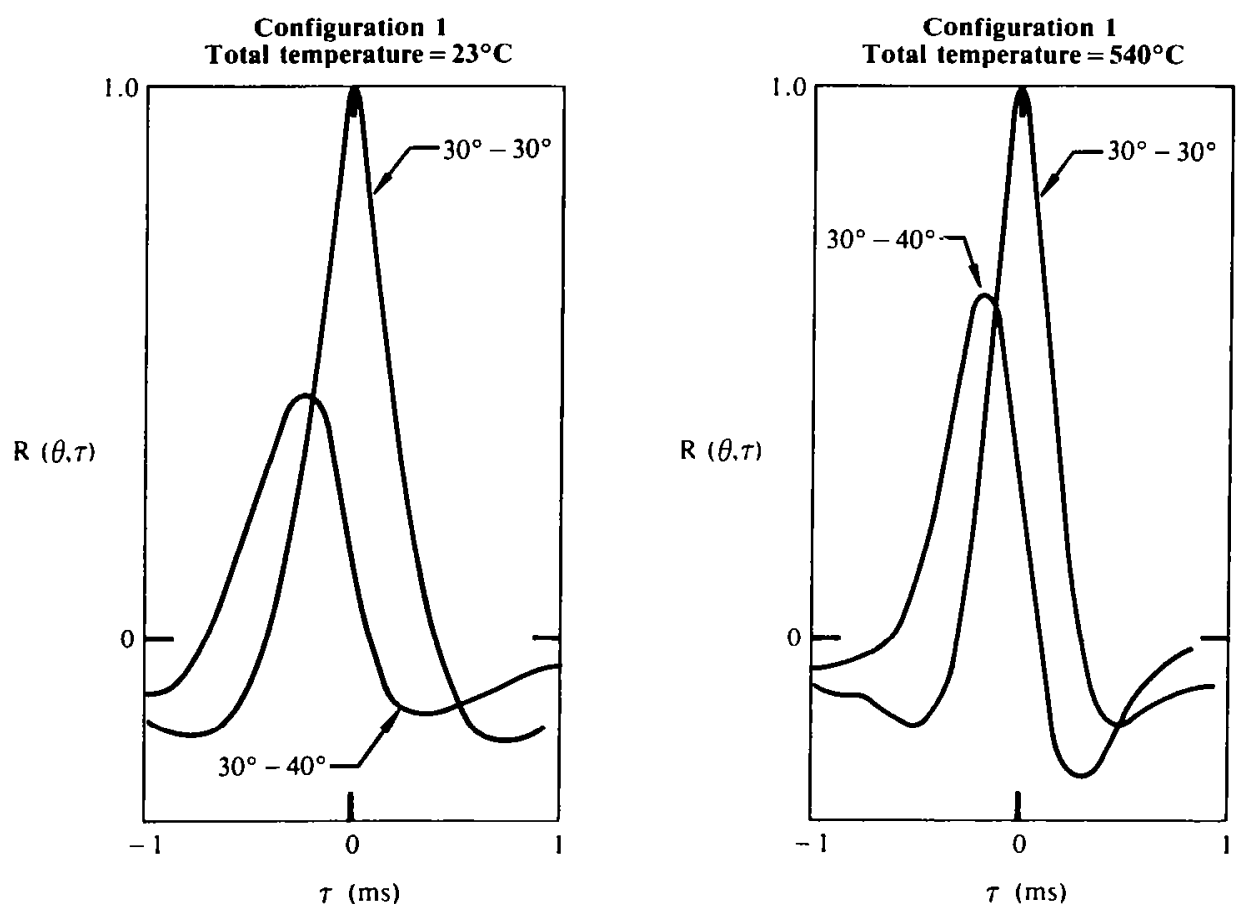

Figure 15. Normalized cross-correlation results for configuration 1 showing effect of the total temperature of the flow at a nozzle pressure ratio of 1.60 . 

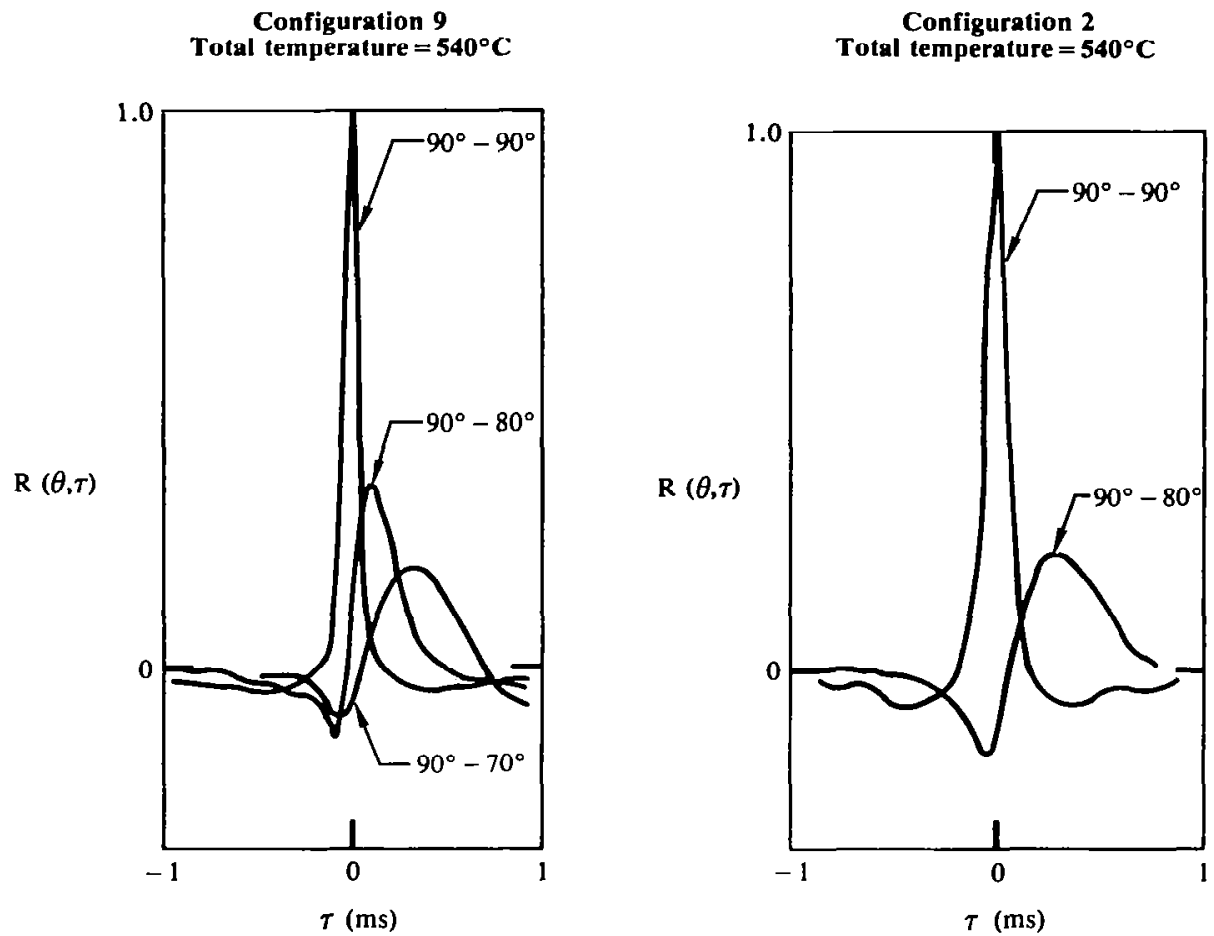

Figure 16. Normalized cross-correlation results for configurations 1 and 9 with a flow total temperature of $540^{\circ} \mathrm{C}$ at a nozzle pressure ratio of 1.60 .

The above correlation plots were obtained from the subsonic pressure ratio testing. Similar results have been obtained for supersonic pressure ratios, as illustrated by Figure 17 .

The fact that the peaks of the correlation curves move progressively to the left for microphone pairs from $30^{\circ}-20^{\circ}$ to $30^{\circ}-30^{\circ}, 30^{\circ}-40^{\circ}$, and $30^{\circ}-50^{\circ}$ (the time delay was incorporated into a signal of the second of the two microphones) indicates that a given signal is sensed earlier by the microphone at the smaller angle. This observation might have been expected, since the acoustic ray paths to the microphones at the smaller angles are 1mmersed for a longer distance in the jet stream, thus speeding the waves traveling at the smaller angles. By the same reasoning, if the center of the microphone circle had been moved in the streamwise direction (1arger $x_{p}$ on Figure 6), the distance from the acoustic emission points to the small-angle microphones would he increased more than that for the large-angle microphones, so that the 

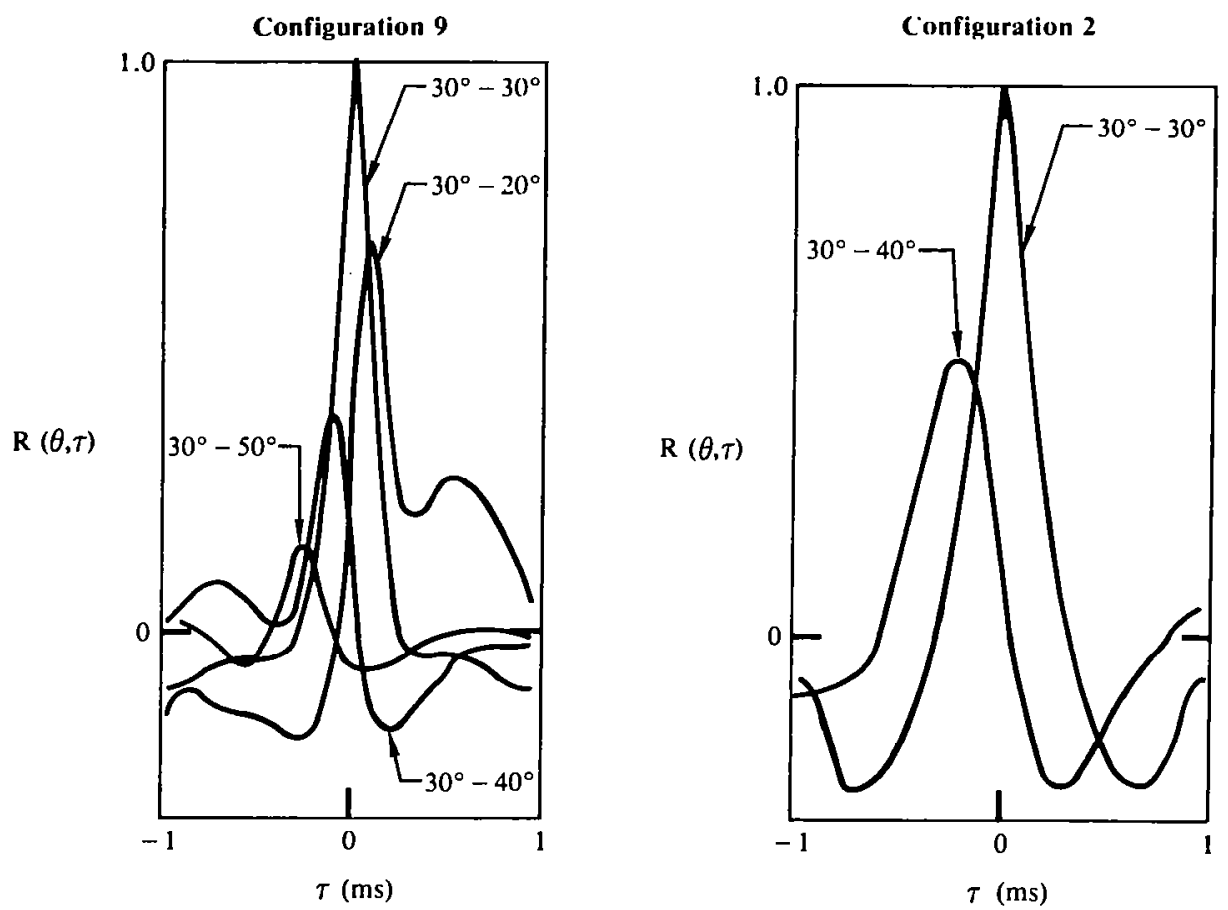

Figure 17. Normalized cross-correlation results for configurations 1 and 9 for various angular separations at a nozzle pressure ratio of 2.80 .

distances between the correlation peaks on Figure 12 would be smaller. Hence, the location of the microphone circle has an effect on the relative location of the correlation peaks.

Jet Noise Suppression with Cold Flow - Figures 18 and 19 are plots showing the noise suppression of several porous plug nozzles with respect to standard converging nozzle sound pressure levels. Figure 18 shows that both the NASA results of Maestre $110^{2}$ and the McDonne11 Douglas Corporation (MDC) results give about the same reductions for subsonic jet speeds even though the NASA and the MDC model design parameters and the nozzle pressure ratios were slightly different. In particular, the plug length to equivalent nozzle diameter ratio of NASA is 7.7, while that of MDC is 3.7 (Configuration 8 ). The sound reductions are plotted for $1 / 3$ octave bands as a function of the Strouhal Number

$$
\mathrm{St}=\frac{\mathrm{f}_{1 / 3^{\mathrm{d}}}}{\mathrm{U}_{\mathrm{i}}},
$$




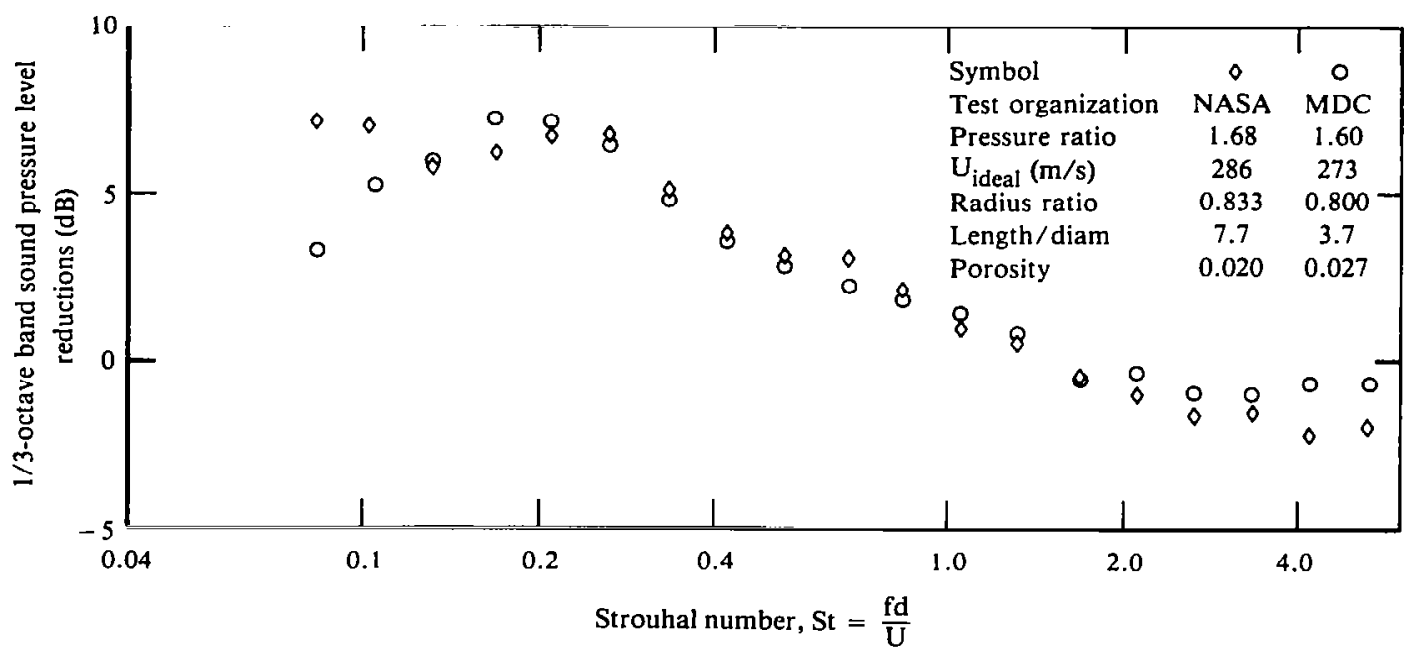

Figure 18. Reductions of sound pressure level by porous plug nozzles. Subsonic jets, $30^{\circ}$ from jet axis.

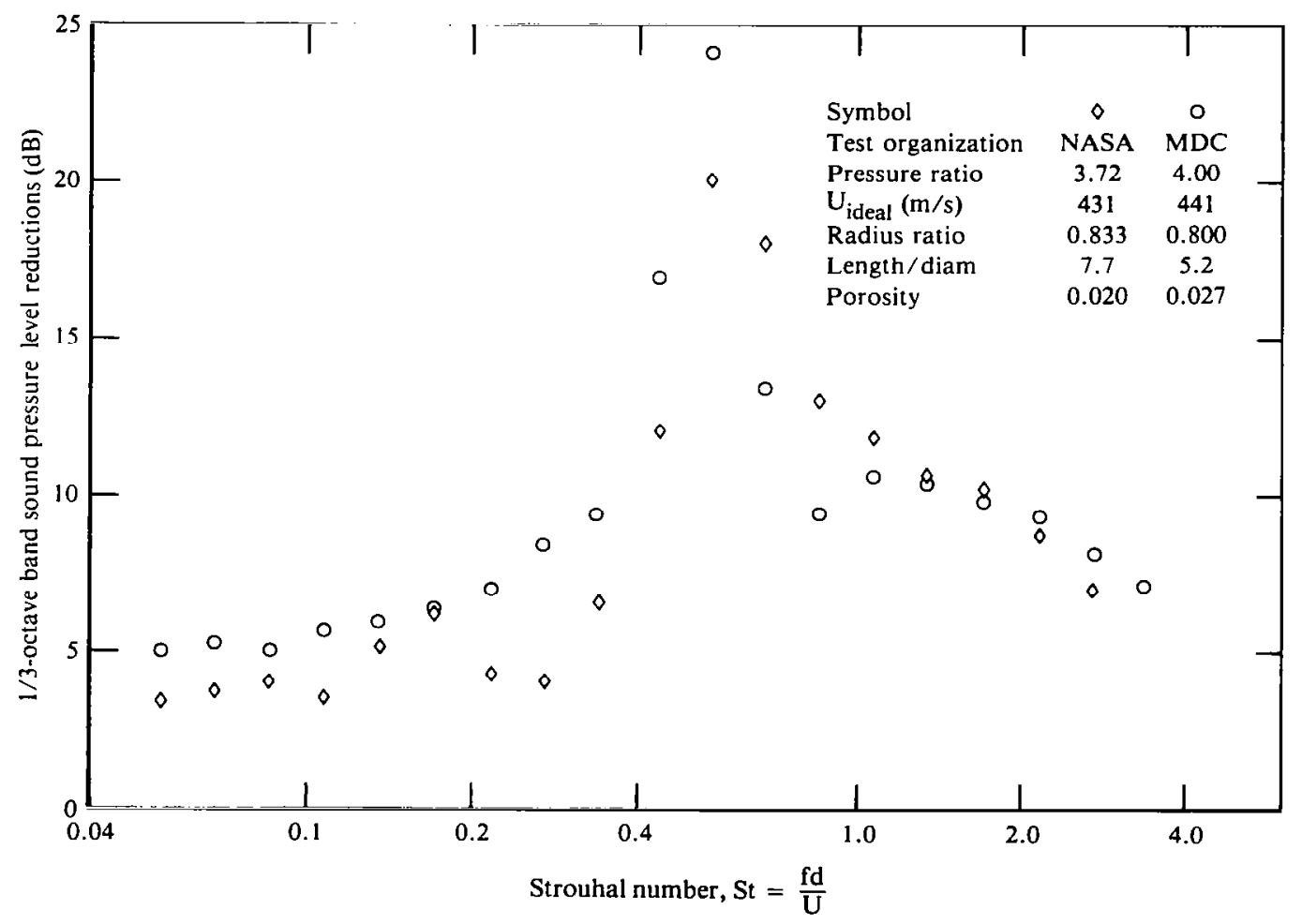

Figure 19. Reductions of sound pressure level by porous plug nozzles. Supersonic jets, $90^{\circ}$ from jet axis. 
where $\mathrm{f}_{1 / 3}$ is the $1 / 3$ octave band center frequency. The peaks in Figure 19 occur at about the same values of St, thereby collapsing the NASA $(d=5.08 \mathrm{~cm})$ and the $\mathrm{MDC}(\mathrm{d}=11.77 \mathrm{~cm})$ data onto a single scale. Figure 19 shows more such data for supersonic pressure ratios near 4.0 and using the MDC Configuration 9, which has a plug-length parameter $l / d$ of 5.2, compared with the NASA value of 7.7. In general, the present (MDC) noise reductions showed good agreement with the NASA data.

The overall sound pressure levels of the present tests are summarized in Tables 6-10. One third octave band spectra and comparisons of the spectra of Configuration 1 with those of other configurations are given in Appendix A. A number of narrow-band spectral plots are presented in Figures 20-25. The plots were made for a filter bandwidth of $60 \mathrm{~Hz}$ and ensemble averaging of 1000 records, each with a 0.025 second sampling time.

Figures 20-22 show data for Configurations 1-10 for Microphone 9 at $90^{\circ}$ to the jet axis and a nozzle pressure ratio of 2.80 ; overall sound pressure levels (OASPL) are given in Table 8 . Although the spectra for Configurations 1-3 are remarkably similar, some differences are apparent. The shock noise tones occur at about the same frequencies, but the tone peak levels vary considerably between the three configurations. The presence of the solid plug and the plug supports on Configuration 3 does not appear to have much effect on the spectra. At frequencies above $10000 \mathrm{~Hz}$, the sound levels of Configuration 2 and 3 are about a decibel below those of Configuration 1 .

For Configurations 4-7 (small diameter porous plugs) the OASPL's are about $10 \mathrm{~dB}$ lower than for the reference nozzles - Configuration 13. Figure 21 shows that no significant narrow-band shock-tones are present. Configuration 5, with twice the porosity of Configurations 4 and 6 , is the quietest, and Configuration 6 is second quietest. Configuration 7 , with the longest plug, is the noisiest.

For Configurations 8-10 (large diameter porous plugs) the OASPL's are still smaller by several dB. Figure 22 shows that Configuration 9 is noisier than Configuration 8 at high frequencies even though 9 is much longer than 8. Configuration 10 is quieter than 8 even at high frequencies, but at low frequencies ( $5000 \mathrm{~Hz}$ model scale) 10 is better 
than 8 or 9, evident1y because 10 has a porosity twice as large as either 8 or 9 . Hence, the porosity acts in the same manner on the large-diameter plugs as on the smal1-diameter plugs.

A direct comparison between Configuration 1, a small radius ratio porous plug (Configuration 5), and a large radius ratio porous plug (Configuration 10) is given in Figures 23-25 for Microphones 3, 9, and 13, respectively.

TABLE 6. OVERALL SOUND PRESSURE LEVELS AND

THRUST COEFFICIENTS.

\begin{tabular}{|c|c|c|c|c|c|c|c|}
\hline \multirow{2}{*}{ Configuration } & \multirow{2}{*}{$\begin{array}{c}\text { Forward } \\
\text { motion } \\
\text { flow }\end{array}$} & \multirow{2}{*}{$\begin{array}{c}\text { Primary } \\
\text { flow total- } \\
\text { temperature }\end{array}$} & \multirow[b]{2}{*}{$\mathbf{C}_{\mathbf{T}}$} & \multicolumn{4}{|c|}{ OASPL at microphone angle (deg) } \\
\hline & & & & 30 & 60 & 90 & 130 \\
\hline 1 & \multirow{3}{*}{ Off } & \multirow{3}{*}{ Ambient } & 0.9906 & 103.1 & 98.8 & 95.3 & 91.5 \\
\hline 2 & & & 0.9918 & 102.2 & 98.3 & 94.4 & 91.5 \\
\hline 3 & & & 0.9759 & 101.8 & 97.9 & 94.6 & 90.6 \\
\hline 4 & \multirow{4}{*}{ Off } & \multirow{4}{*}{ Ambient } & 0.9703 & 101.1 & 97.6 & 94.3 & 90.0 \\
\hline 5 & & & 0.9736 & 101.2 & 97.5 & 94.1 & 90.7 \\
\hline 6 & & & 0.9770 & 101.0 & 97.7 & 94.3 & 90.3 \\
\hline 7 & & & 0.9682 & 101.1 & 97.6 & 94.4 & 90.3 \\
\hline 8 & \multirow{3}{*}{ Off } & \multirow{3}{*}{ Ambient } & 0.9557 & 99.1 & 96.1 & 92.9 & 88.8 \\
\hline 9 & & & 0.9440 & 98.8 & 96.7 & 93.8 & 90.1 \\
\hline 10 & & & 0.9344 & 98.8 & 97.1 & 95.2 & 93.4 \\
\hline 1 & \multirow{3}{*}{ Off } & \multirow{3}{*}{$370^{\circ} \mathrm{C}$} & 0.9820 & 117.3 & 110.8 & 104.0 & 98.2 \\
\hline 4 & & & 0.9599 & 114.2 & 109.6 & 103.3 & 96.9 \\
\hline 9 & & & 0.9230 & 109.9 & 107.9 & 102.1 & 95.8 \\
\hline $1^{*}$ & \multirow{3}{*}{ Off } & \multirow{3}{*}{$540^{\circ} \mathrm{C}$} & 0.9853 & 121.4 & 113.7 & 106.6 & 101.0 \\
\hline 4 & & & 0.9627 & 117.0 & 112.6 & 105.7 & 98.9 \\
\hline 9 & & & 0.9073 & 113.7 & 111.2 & 105.3 & 97.8 \\
\hline 1 & \multirow{3}{*}{ On } & \multirow{3}{*}{ Ambient } & & 97.5 & 93.6 & 89.8 & 85.8 \\
\hline 4 & & & & 96.2 & 93.6 & 89.8 & 86.2 \\
\hline 9 & & & & 93.6 & 92.6 & 89.6 & 85.9 \\
\hline $4^{*}$ & \multirow{4}{*}{ Off } & \multirow{4}{*}{ Ambient } & 0.9825 & 101.0 & 97.6 & 93.9 & 91.2 \\
\hline $4^{*}$, Rakes In & & & 0.9698 & 101.2 & 97.8 & 94.3 & 91.0 \\
\hline $1^{*}$ & & & 0.9906 & 102.4 & 98.3 & 94.8 & 91.8 \\
\hline $1^{*}$, Rakes In & & & 0.9950 & 102.4 & 98.3 & 94.8 & 92.0 \\
\hline
\end{tabular}

Frequency range $=0.178$ to $89.8 \mathrm{kHz}$

Nozzle pressure ratio $=1.6$ 
TABLE 7. OVERALL SOUND PRESSURE LEVELS AND THRUST COEFFICIENTS.

\begin{tabular}{|c|c|c|c|c|c|c|c|}
\hline \multirow{2}{*}{ Configuration } & \multirow{2}{*}{$\begin{array}{l}\text { Forward } \\
\text { motion } \\
\text { flow }\end{array}$} & \multirow{2}{*}{$\begin{array}{l}\text { Primary } \\
\text { flow total- } \\
\text { temperature }\end{array}$} & \multirow[b]{2}{*}{$\mathbf{C}_{\mathbf{T}}$} & \multicolumn{4}{|c|}{ OASPL at microphone angle (deg) } \\
\hline & & & & 30 & 60 & 90 & 130 \\
\hline 1 & \multirow{3}{*}{ Off } & \multirow{3}{*}{ Ambient } & 0.9965 & 119.4 & 113.1 & 110.5 & 112.2 \\
\hline 2 & & & 0.9946 & 117.2 & 112.7 & 110.1 & 111.6 \\
\hline 3 & & & 0.9863 & 113.1 & 107.0 & 103.6 & 101.2 \\
\hline 4 & \multirow{4}{*}{ Off } & \multirow{4}{*}{ Ambient } & 0.9798 & 111.5 & 107.0 & 105.6 & 106.1 \\
\hline 5 & & & 0.9775 & 111.7 & 106.8 & 104.4 & 104.4 \\
\hline 6 & & & 0.9817 & 111.7 & 107.1 & 105.2 & 105.8 \\
\hline 7 & & & 0.9757 & 111.4 & 107.0 & 105.5 & 105.4 \\
\hline 8 & \multirow{3}{*}{ Off } & \multirow{3}{*}{ Ambient } & 0.9687 & 109.9 & 104.9 & 102.6 & 101.4 \\
\hline 9 & & & 0.9545 & 108.8 & 105.2 & 102.9 & 101.2 \\
\hline 10 & & & 0.9471 & 108.6 & 105.2 & 102.2 & 99.6 \\
\hline 1 & \multirow{3}{*}{ Off } & \multirow{3}{*}{$370^{\circ} \mathrm{C}$} & 0.9951 & 127.0 & 121.8 & 115.3 & 111.7 \\
\hline 4 & & & 0.9750 & 125.5 & 117.5 & 110.4 & 106.5 \\
\hline 9 & & & 0.9400 & 120.5 & 115.4 & 108.4 & 102.7 \\
\hline $1^{*}$ & \multirow{3}{*}{ Off } & \multirow{3}{*}{$540^{\circ} \mathrm{C}$} & 0.9938 & 129.6 & 121.8 & 113.3 & 108.9 \\
\hline 4 & & & 0.9735 & 126.9 & 120.3 & 112.2 & 106.7 \\
\hline 9 & & & 0.9353 & 123.5 & 118.7 & 110.6 & 104.2 \\
\hline 1 & \multirow{3}{*}{ On } & \multirow{3}{*}{ Ambient } & & 111.9 & 107.6 & 107.0 & 105.4 \\
\hline 4 & & & & 108.3 & 104.2 & 103.2 & 104.9 \\
\hline 9 & & & & 105.1 & 102.2 & 100.8 & 99.8 \\
\hline $4^{*}$ & \multirow{4}{*}{ Off } & \multirow{4}{*}{ Ambient } & 0.9813 & 111.7 & 107.0 & 105.4 & 106.6 \\
\hline $4^{*}$, Rakes In & & & 0.9780 & 111.9 & 107.4 & 105.7 & 106.5 \\
\hline & & & 0.9975 & 115.6 & 110.1 & 108.3 & 109.2 \\
\hline $1^{*}$, Rakes In & & & 0.9983 & 115.5 & 110.1 & 108.2 & 109.1 \\
\hline
\end{tabular}

Frequency range $=0.178$ to $89.8 \mathrm{kHz}$

Nozzle pressure ratio $=\mathbf{2 . 2}$ 
TABLE 8. OVERALL SOUND PRESSURE LEVELS AND THRUST COEFFICIENTS.

\begin{tabular}{|c|c|c|c|c|c|c|c|}
\hline \multirow{2}{*}{ Configuration } & \multirow{2}{*}{$\begin{array}{l}\text { Forward } \\
\text { motion } \\
\text { flow }\end{array}$} & \multirow{2}{*}{$\begin{array}{c}\text { Primary } \\
\text { flow total- } \\
\text { temperature }\end{array}$} & \multirow[b]{2}{*}{$\mathbf{C}_{\mathbf{T}}$} & \multicolumn{4}{|c|}{ OASPL at microphone angle (deg) } \\
\hline & & & & 30 & 60 & 90 & 130 \\
\hline 1 & \multirow{3}{*}{ Off } & \multirow{3}{*}{ Ambient } & 0.9954 & 122.8 & 120.1 & 122.4 & 118.5 \\
\hline 2 & & & 0.9963 & 123.5 & 119.5 & 122.1 & 121.9 \\
\hline 3 & & & 0.9821 & 123.1 & 118.4 & 121.4 & 118.3 \\
\hline 4 & \multirow{4}{*}{ Off } & \multirow{4}{*}{ Ambient } & 0.9789 & 117.2 & 113.1 & 111.8 & 112.0 \\
\hline 5 & & & 0.9744 & 117.2 & 112.0 & 110.2 & 110.0 \\
\hline 6 & & & 0.9795 & 117.4 & 112.7 & 111.2 & 111.7 \\
\hline 7 & & & 0.9742 & 117.0 & 113.2 & 112.8 & 113.4 \\
\hline 8 & \multirow{3}{*}{ Off } & \multirow{3}{*}{ Ambient } & 0.9711 & 116.1 & 110.4 & 108.9 & 109.6 \\
\hline 9 & & & 0.9592 & 114.8 & 110.9 & 109.0 & 108.0 \\
\hline 10 & & & 0.9492 & 114.4 & 109.6 & 106.8 & 104.4 \\
\hline 1 & \multirow{3}{*}{ Off } & \multirow{3}{*}{$370^{\circ} \mathrm{C}$} & 0.9955 & 134.9 & 126.7 & 122.1 & 121.3 \\
\hline 4 & & & 0.9776 & 130.1 & 121.9 & 114.3 & 111.5 \\
\hline 9 & & & 0.9470 & 125.9 & 119.5 & 111.9 & 107.7 \\
\hline $1^{*}$ & \multirow{3}{*}{ Off } & \multirow{3}{*}{$540^{\circ} \mathrm{C}$} & 0.9907 & 132.1 & 126.5 & 120.5 & 119.6 \\
\hline 4 & & & 0.9777 & 130.3 & 124.6 & 116.3 & 113.3 \\
\hline 9 & & & 0.9445 & 127.4 & 122.7 & 114.4 & 108.6 \\
\hline 1 & \multirow{3}{*}{ On } & \multirow{3}{*}{ Ambient } & & 123.0 & 118.4 & 124.4 & 117.1 \\
\hline 4 & & & & 115.3 & 112.3 & 112.3 & 113.4 \\
\hline 9 & & & & 111.9 & 108.6 & 107.8 & 107.6 \\
\hline $4^{*}$ & \multirow{4}{*}{ Off } & \multirow{4}{*}{ Ambient } & 0.9796 & 117.2 & 112.8 & 111.2 & 111.7 \\
\hline $4^{*}$, Rakes In & & & 0.9782 & 117.3 & 112.5 & 111.4 & 111.8 \\
\hline $1^{*}$ & & & 0.9956 & 122.5 & 117.9 & 121.3 & 118.1 \\
\hline $1^{*}$, Rakes In & & & 0.9952 & 112.7 & 118.1 & 122.5 & 118.2 \\
\hline
\end{tabular}

Frequency range $=0.178$ to $89.8 \mathrm{kHz}$

Nozzle pressure ratio $=2.8$ 
TABLE 9. OVERALL SOUND PRESSURE LEVELS AND

THRUST COEFFICIENTS.

\begin{tabular}{|c|c|c|c|c|c|c|c|}
\hline \multirow[t]{2}{*}{ Configuration } & \multirow{2}{*}{$\begin{array}{c}\text { Forward } \\
\text { motion } \\
\text { flow }\end{array}$} & \multirow{2}{*}{$\begin{array}{c}\text { Primary } \\
\text { flow total- } \\
\text { temperature }\end{array}$} & \multirow{2}{*}{$\mathrm{C}_{\mathrm{T}}$} & \multicolumn{4}{|c|}{ OASPL at microphone angle (deg) } \\
\hline & & & & 30 & 60 & 90 & 130 \\
\hline 1 & \multirow{3}{*}{ Off } & \multirow{3}{*}{ Ambient } & 0.9888 & 127.6 & 122.9 & 127.1 & 124.2 \\
\hline 2 & & & 0.9906 & 128.1 & 123.4 & 127.2 & 125.7 \\
\hline 3 & & & 0.9820 & 126.1 & 118.7 & 118.7 & 118.1 \\
\hline 4 & \multirow{4}{*}{ Off } & \multirow{4}{*}{ Ambient } & 0.9724 & 121.1 & 117.4 & 116.3 & 116.3 \\
\hline 5 & & & 0.9672 & 120.7 & 114.8 & 113.0 & 112.5 \\
\hline 6 & & & 0.9727 & 121.3 & 116.8 & 113.7 & 112.9 \\
\hline 7 & & & 0.9702 & 120.7 & 116.3 & 114.0 & 113.9 \\
\hline 8 & \multirow{3}{*}{ Off } & \multirow{3}{*}{ Ambient } & 0.9659 & 119.9 & 113.6 & 111.9 & 111.4 \\
\hline 9 & & & 0.9593 & 118.8 & 113.7 & 111.7 & 110.3 \\
\hline 10 & & & 0.9453 & 118.0 & 112.4 & 109.6 & 107.2 \\
\hline 1 & \multirow{3}{*}{ Off } & \multirow{3}{*}{$370^{\circ} \mathrm{C}$} & 0.9888 & 139.1 & 130.0 & 126.3 & 126.1 \\
\hline 4 & & & 0.9723 & 132.5 & 124.6 & 118.3 & 117.0 \\
\hline 9 & & & 0.9468 & 128.8 & 122.1 & 114.6 & 110.6 \\
\hline$I^{*}$ & \multirow{3}{*}{ Off } & \multirow{3}{*}{$540^{\circ} \mathrm{C}$} & 0.9824 & 134.3 & 129.1 & 124.6 & 123.1 \\
\hline 4 & & & 0.9700 & 132.3 & 127.1 & 120.0 & 117.9 \\
\hline 9 & & & 0.9429 & 130.2 & 125.2 & 116.8 & 110.9 \\
\hline 1 & \multirow{3}{*}{ On } & \multirow{3}{*}{ Ambient } & & 126.9 & 123.5 & 127.3 & 120.4 \\
\hline 4 & & & & 119.5 & 117.8 & 118.5 & 118.7 \\
\hline 9 & & & & 116.6 & 115.6 & 114.1 & 113.4 \\
\hline $4^{*}$ & \multirow{4}{*}{ Off } & \multirow{4}{*}{ Ambient } & 0.9727 & 121.2 & 116.8 & 115.2 & 115.3 \\
\hline $4^{*}$, Rakes In & & & 0.9744 & 121.2 & 117.1 & 115.8 & 115.8 \\
\hline & & & 0.9888 & 126.2 & 121.8 & 122.5 & 121.7 \\
\hline $1^{*}$, Rakes In & & & 0.9871 & 126.3 & 123.7 & 123.2 & 122.5 \\
\hline
\end{tabular}

Frequency range $=0.178$ to $89.8 \mathrm{kHz}$

Nozzle pressure ratio $=3.4$ 
TABLE 10. OVERALL SOUND PRESSURE LEVELS AND THRUST COEFFICIENTS.

\begin{tabular}{|c|c|c|c|c|c|c|c|}
\hline \multirow{2}{*}{ Configuration } & \multirow{2}{*}{$\begin{array}{l}\text { Forward } \\
\text { motion } \\
\text { flow }\end{array}$} & \multirow{2}{*}{$\begin{array}{c}\text { Primary } \\
\text { flow total- } \\
\text { temperature }\end{array}$} & \multirow[b]{2}{*}{$\mathbf{C}_{\mathbf{T}}$} & \multicolumn{4}{|c|}{ OASPL at microphone angle (deg) } \\
\hline & & & & 30 & 60 & 90 & 130 \\
\hline 1 & \multirow{3}{*}{ Off } & \multirow{3}{*}{ Ambient } & 0.9828 & 129.1 & 123.5 & 124.4 & 124.0 \\
\hline 2 & & & 0.9898 & 129.5 & 124.4 & 125.3 & 127.0 \\
\hline 3 & & & 0.9838 & 128.9 & 119.3 & 118.8 & 117.0 \\
\hline 4 & \multirow{4}{*}{ Off } & \multirow{4}{*}{ Ambient } & 0.9647 & 124.6 & 119.9 & 119.2 & 118.2 \\
\hline 5 & & & 0.9628 & 123.7 & 117.8 & 116.5 & 115.2 \\
\hline 6 & & & 0.9659 & 124.6 & 119.4 & 117.2 & 116.0 \\
\hline 7 & & & 0.9653 & 123.7 & 119.2 & 117.6 & 116.5 \\
\hline 8 & \multirow{3}{*}{ Off } & \multirow{3}{*}{ Ambient } & 0.9632 & 123.3 & 116.8 & 115.1 & 113.9 \\
\hline 9 & & & 0.9585 & 122.2 & 116.5 & 113.2 & 111.1 \\
\hline 10 & & & 0.9427 & 121.0 & 114.4 & 111.8 & 109.1 \\
\hline 1 & \multirow{3}{*}{ Off } & \multirow{3}{*}{$370^{\circ} \mathrm{C}$} & 0.9831 & 136.8 & 128.8 & 126.5 & 125.2 \\
\hline 4 & & & 0.9645 & 134.5 & 126.8 & 121.9 & 120.5 \\
\hline 9 & & & 0.9464 & 131.2 & 123.8 & 116.7 & 113.2 \\
\hline $1^{*}$ & \multirow{3}{*}{ Off } & \multirow{3}{*}{$540^{\circ} \mathrm{C}$} & 0.9766 & 135.2 & 130.3 & 126.3 & 124.5 \\
\hline 4 & & & & & & & \\
\hline 9 & & & 0.9409 & 132.1 & 126.8 & 118.7 & 113.2 \\
\hline 1 & \multirow{3}{*}{ On } & \multirow{3}{*}{ Ambient } & & 127.4 & 123.8 & 125.3 & 124.2 \\
\hline 4 & & & & 123.0 & 119.2 & 119.7 & 119.1 \\
\hline 9 & & & & 120.1 & 115.6 & 114.1 & 113.4 \\
\hline $4^{*}$ & \multirow{4}{*}{ Off } & \multirow{4}{*}{ Ambient } & 0.9630 & 124.3 & 119.6 & 118.1 & 117.1 \\
\hline $4^{*}$, Rakes In & & & 0.9662 & 124.4 & 119.7 & 118.0 & 117.1 \\
\hline & & & 0.9797 & 127.8 & 123.8 & 123.2 & 122.7 \\
\hline $1^{*}$, Rakes In & & & 0.9783 & 127.6 & 123.7 & 123.2 & 122.5 \\
\hline
\end{tabular}

Frequency range $=0.178$ to $89.8 \mathrm{kHz}$

Nozzle pressure ratio $=4.0$ 


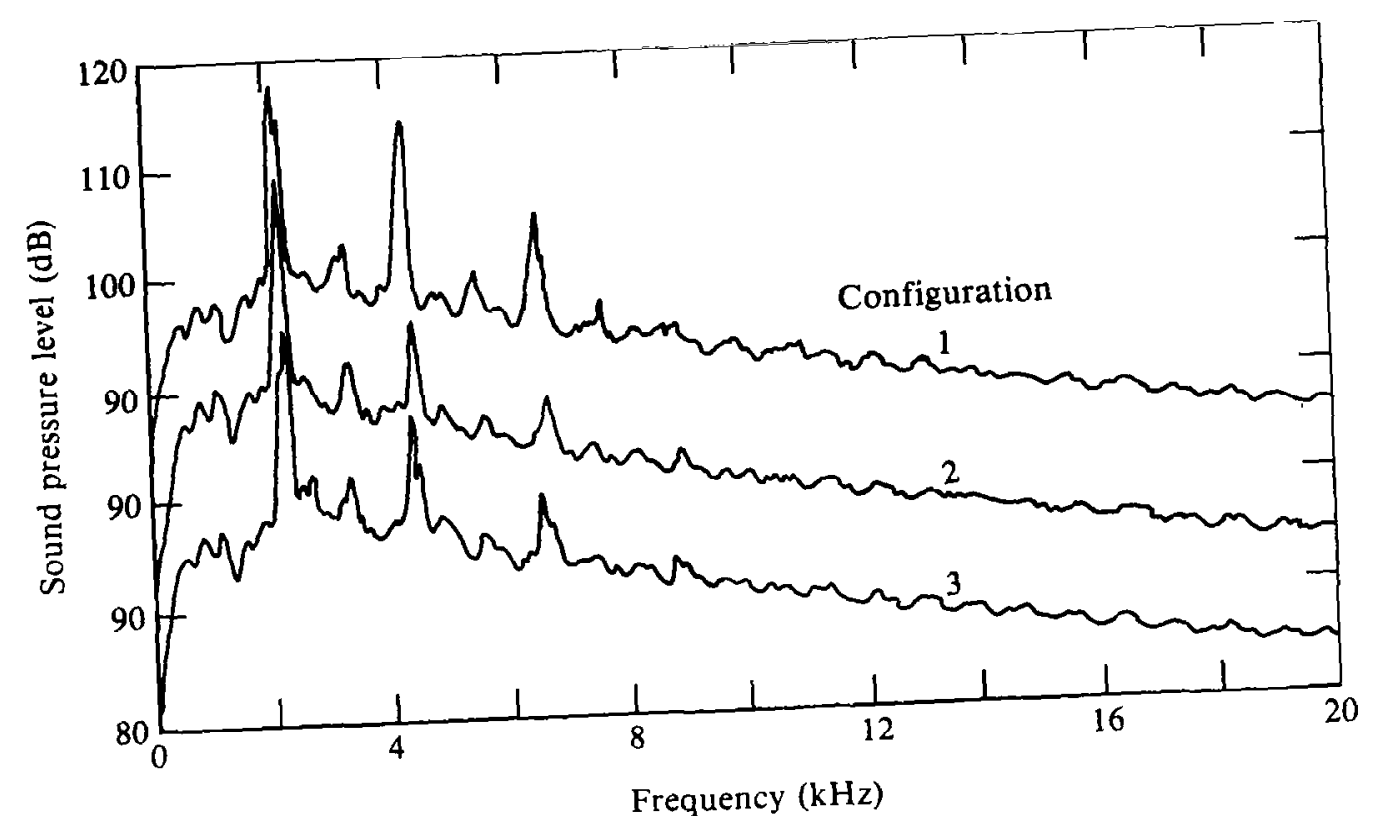

Figure 20. Narrow-band spectra for configurations 1, 2 and 3;

microphone 9, pressure ratio $=2.8$, and bandwidth $=60 \mathrm{~Hz}$.

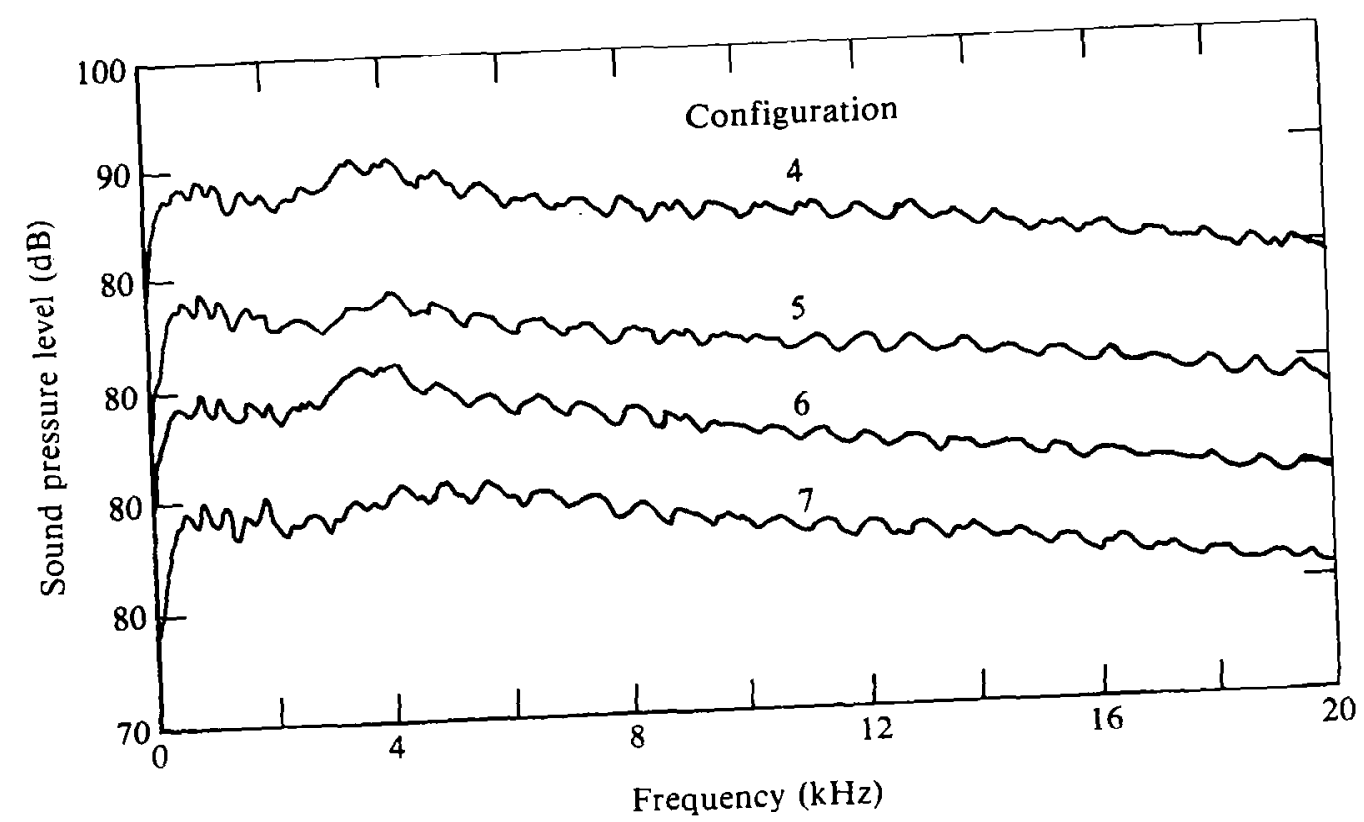
Figure 21. Narrow-band spectra for configurations $4,5,6$, and 7 ;
microphone 9 , pressure ratio $=2.8$, and bandwidth $=60 \mathrm{~Hz}$. 


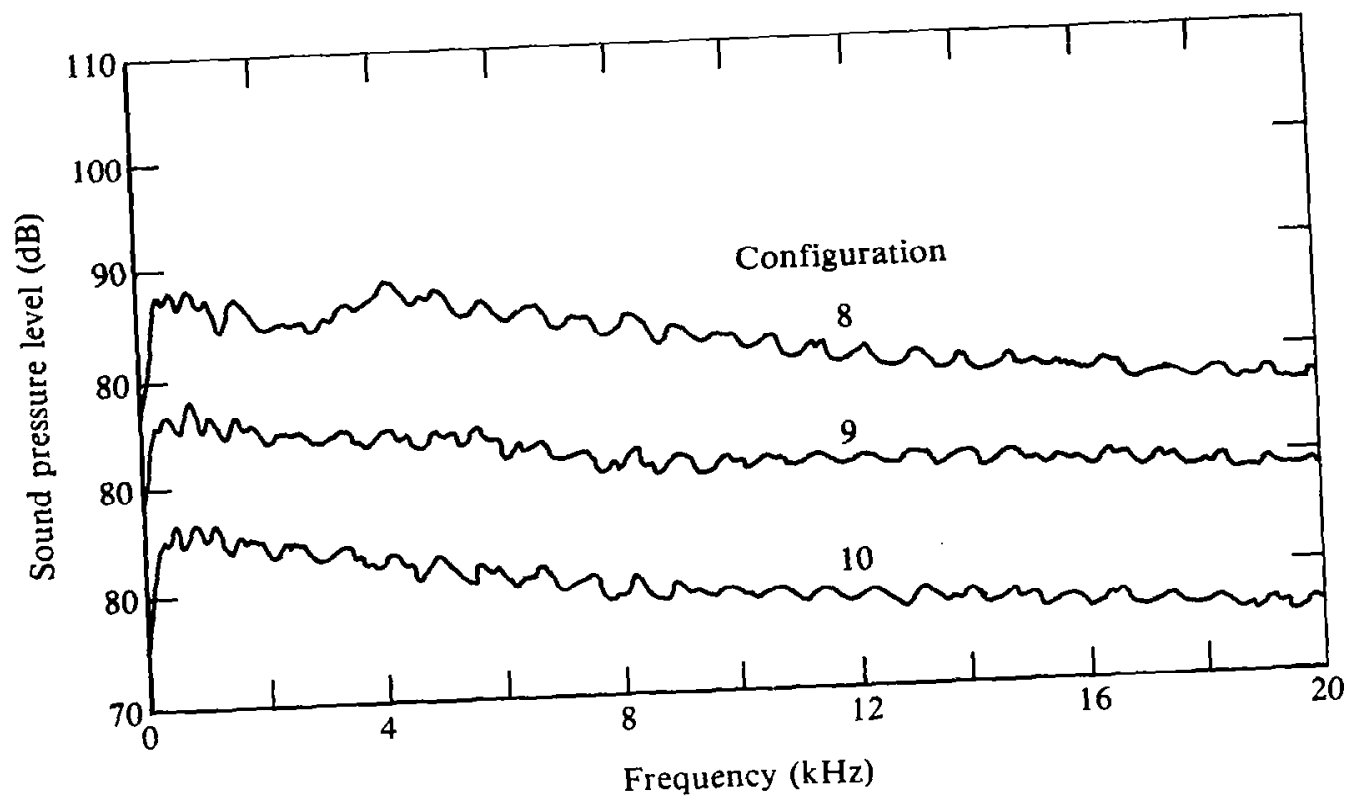

Figure 22. Narrow-band spectra for configurations 8, 9, and 10; microphone 9, pressure ratio $=2.8$, and bandwidth $=60 \mathrm{~Hz}$.

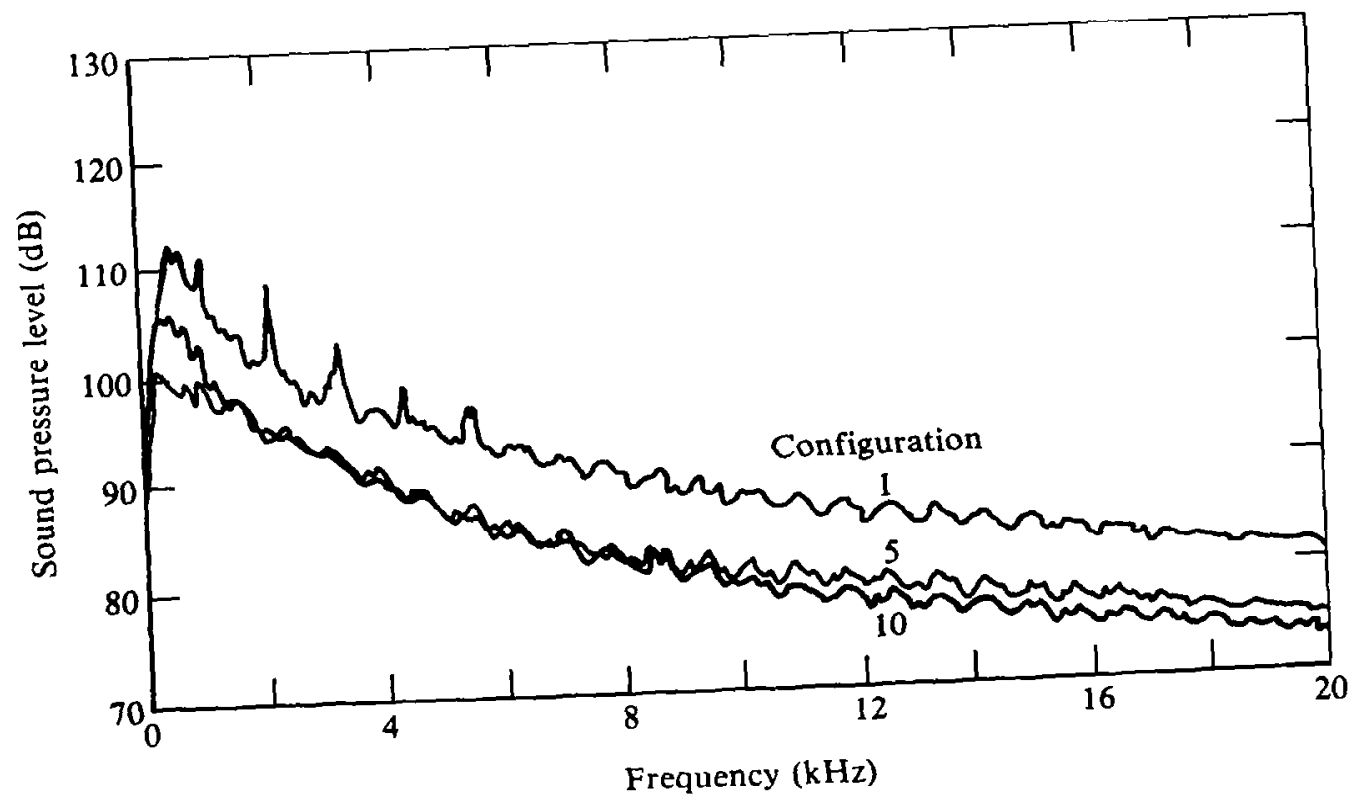

Figure 23. Narrow-band spectra for configurations 1,5 , and 10; microphone 3 , pressure ratio $=2.8$, and bandwidth $=60 \mathrm{~Hz}$. 


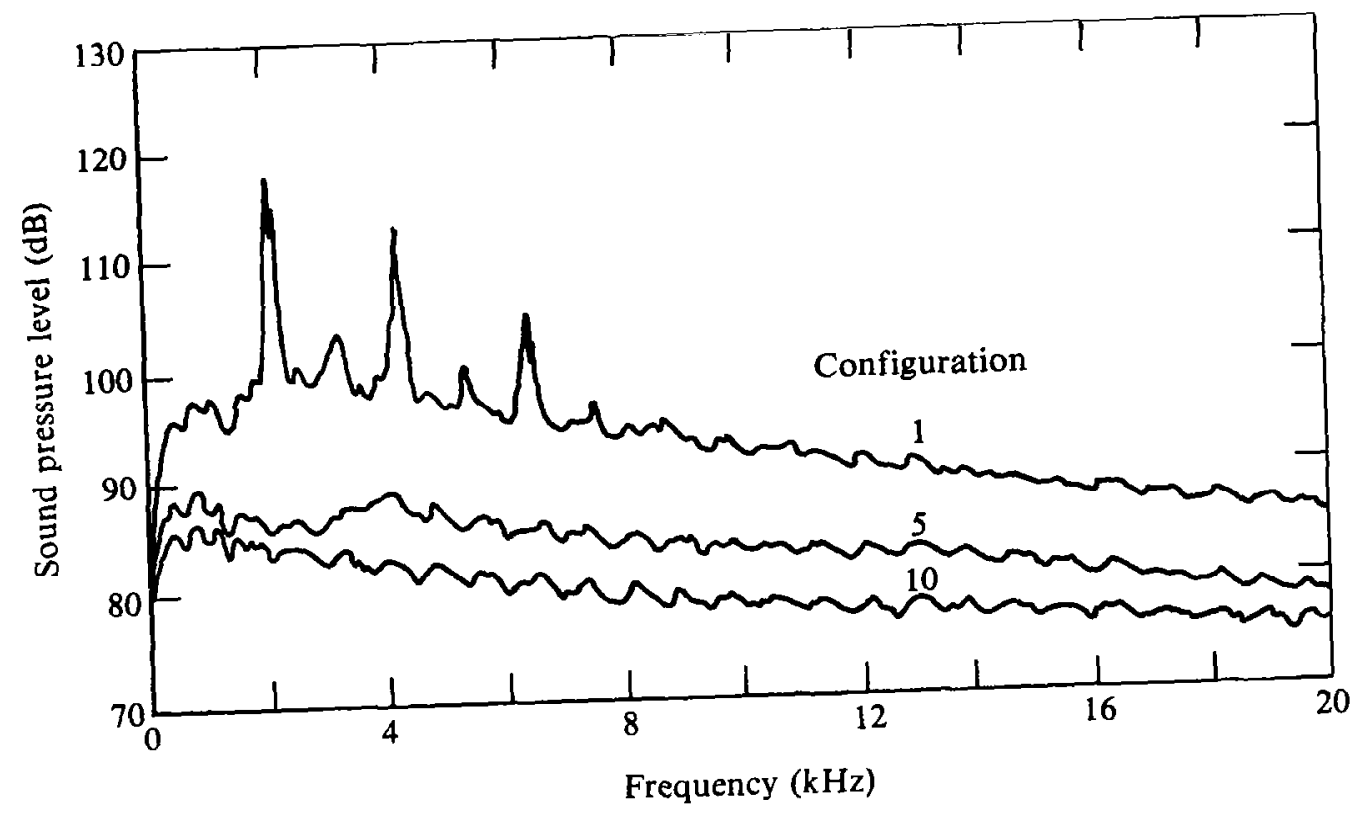

Figure 24. Narrow-band spectra for configurations 1,5 , and 10 ;
microphone 9 , pressure ratio $=2.8$, and bandwidth $=60 \mathrm{~Hz}$.

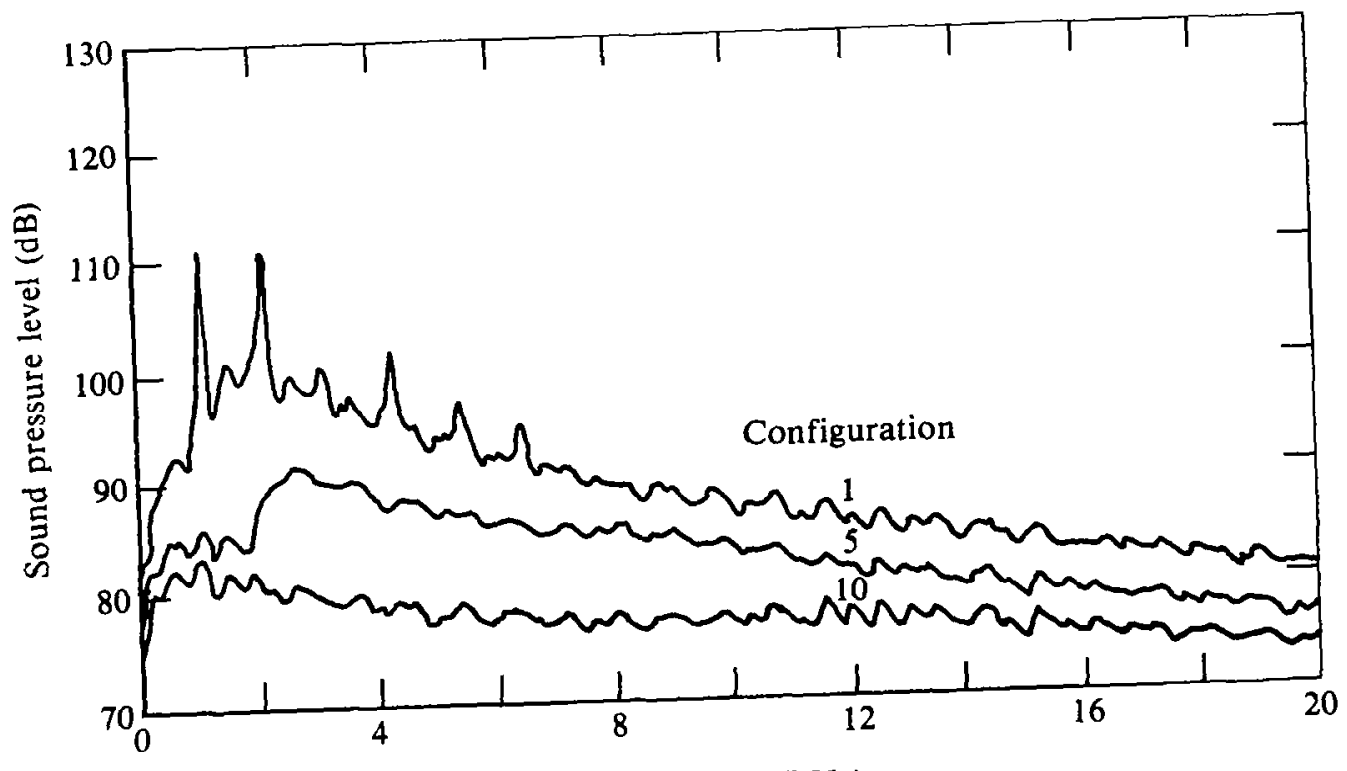

Frequency $(\mathrm{kHz})$

Figure 25. Narrow-band spectra for configurations 1, 5, and 10; microphone 13 , pressure ratio $=2.8$, and bandwidth $=60 \mathrm{~Hz}$. 
Porous plug nolse suppression data, expressed as reductions in OASPL calculated by comparing Configuration 1 OASPL data with either Configuration 4 or Configuration 9 data, are shown in Tables 11-15. Figures A9-A36 show one-third octave band spectral plots with the suppression generated by porous plug Configurations 4-10 as compared with spectra for Configuration 1.

At a pressure ratio of 3.40 , the porous plugs give somewhat greater suppression than at 2.80 , as shown in Tables 13 and 14 . However, at a pressure ratio of 4.00 , the suppressors do not work as well as at 2.80 (see Tables 13 and 15).

At a pressure ratio of 2.20 , the ideal jet Mach number is only slightly supersonic (1.124) and the suppression is less than at 2.80 , probably because of the decreased shock noise at the lower pressure ratio. Table 7 shows that an anomaly occurs with Configuration 3; its jet noise is less at Microphones 9 and 13 than for Configurations 4-7. The solid plug flow conditions evidently suppressed the noise by some unknown mechanism. Configuration 3 also was tested with forward motion flow with similar results.

\section{TABLE 11. OVERALL SOUND PRESSURE LEVEL SUPPRESSION FOR CONFIGURATIONS 4 AND 9 IN COMPARISON WITH CONFIGURATION 1.}

\begin{tabular}{|c|c|c|c|c|c|c|}
\hline \multirow[t]{2}{*}{ Configuration } & \multirow{2}{*}{$\begin{array}{c}\text { Forward } \\
\text { motion } \\
\text { flow }\end{array}$} & \multirow{2}{*}{$\begin{array}{c}\text { Primary } \\
\text { flow total- } \\
\text { temperature }\end{array}$} & \multicolumn{4}{|c|}{$\triangle$ OASPL at microphone angle (deg) } \\
\hline & & & 30 & 60 & 90 & 130 \\
\hline $\begin{array}{l}1-4 \\
1-9\end{array}$ & Off & Ambient & $\begin{array}{l}2.0 \\
4.3\end{array}$ & $\begin{array}{l}1.2 \\
2.1\end{array}$ & $\begin{array}{l}1.0 \\
1.5\end{array}$ & $\begin{array}{l}1.5 \\
1.4\end{array}$ \\
\hline $\begin{array}{l}1-4 \\
1-9\end{array}$ & Off & $370^{\circ} \mathrm{C}$ & $\begin{array}{l}3.1 \\
7.4\end{array}$ & $\begin{array}{l}1.2 \\
2.9\end{array}$ & $\begin{array}{l}0.7 \\
1.9\end{array}$ & $\begin{array}{l}1.3 \\
2.4\end{array}$ \\
\hline $\begin{array}{l}1^{*}-4 \\
1^{*}-9\end{array}$ & Off & $540^{\circ} \mathrm{C}$ & $\begin{array}{l}4.4 \\
7.7\end{array}$ & $\begin{array}{l}1.1 \\
2.5\end{array}$ & $\begin{array}{l}0.9 \\
1.3\end{array}$ & $\begin{array}{l}2.1 \\
3.2\end{array}$ \\
\hline $\begin{array}{l}1-4 \\
1-9\end{array}$ & On & Ambient & $\begin{array}{l}1.3 \\
3.5\end{array}$ & $\begin{array}{l}0.0 \\
1.0\end{array}$ & $\begin{array}{l}0.0 \\
0.2\end{array}$ & $\begin{array}{l}-0.4 \\
-0.1\end{array}$ \\
\hline
\end{tabular}

Frequency range $=0.178$ to $89.8 \mathrm{kHz}$

Nozzle pressure ratio $=1.6$ 


\section{TABLE 12. OVERALL SOUND PRESSURE LEVEL SUPPRESSION FOR CONFIGURATIONS 4 AND 9 IN COMPARISON WITH CONFIGURATION 1.}

\begin{tabular}{|c|c|c|c|c|c|c|}
\hline \multirow{2}{*}{ Configuration } & \multirow{2}{*}{$\begin{array}{l}\text { Forward } \\
\text { motion } \\
\text { flow }\end{array}$} & \multirow{2}{*}{$\begin{array}{c}\text { Primary } \\
\text { flow total- } \\
\text { temperature }\end{array}$} & \multicolumn{4}{|c|}{$\triangle$ OASPL at microphone angle (deg) } \\
\hline & & & 30 & 60 & 90 & 130 \\
\hline $\begin{array}{l}1-4 \\
1-9\end{array}$ & Off & Ambient & $\begin{array}{r}7.9 \\
10.6\end{array}$ & $\begin{array}{l}6.1 \\
7.9\end{array}$ & $\begin{array}{l}4.9 \\
7.6\end{array}$ & $\begin{array}{r}6.1 \\
11.0\end{array}$ \\
\hline $\begin{array}{l}1-4 \\
1-9\end{array}$ & Off & $370^{\circ} \mathrm{C}$ & $\begin{array}{l}1.5 \\
6.5\end{array}$ & $\begin{array}{l}4.3 \\
6.4\end{array}$ & $\begin{array}{l}4.9 \\
6.9\end{array}$ & $\begin{array}{l}5.2 \\
9.0\end{array}$ \\
\hline $\begin{array}{l}1^{*}-4 \\
1^{*}-9\end{array}$ & Off & $540^{\circ} \mathrm{C}$ & $\begin{array}{l}2.7 \\
6.1\end{array}$ & $\begin{array}{l}1.5 \\
3.1\end{array}$ & $\begin{array}{l}1.1 \\
2.7\end{array}$ & $\begin{array}{l}2.2 \\
4.7\end{array}$ \\
\hline $\begin{array}{l}1-4 \\
1-9\end{array}$ & On & Ambient & $\begin{array}{l}3.6 \\
6.8\end{array}$ & $\begin{array}{l}3.4 \\
5.4\end{array}$ & $\begin{array}{l}3.8 \\
6.2\end{array}$ & $\begin{array}{l}0.5 \\
5.6\end{array}$ \\
\hline
\end{tabular}

Frequency range $=0.178$ to $89.8 \mathrm{kHz}$

Nozzle pressure ratio $=2.2$

TABLE 13. OVERALL SOUND PRESSURE LEVEL SUPPRESSION FOR CONFIGURATIONS 4 AND 9 IN COMPARISON WITH CONFIGURATION 1.

\begin{tabular}{|c|c|c|c|c|c|c|}
\hline \multirow{2}{*}{ Configuration } & \multirow{2}{*}{$\begin{array}{l}\text { Forward } \\
\text { motion } \\
\text { flow }\end{array}$} & \multirow{2}{*}{$\begin{array}{c}\text { Primary } \\
\text { flow total- } \\
\text { temperature }\end{array}$} & \multicolumn{4}{|c|}{$\triangle$ OASPL at microphone angle (deg) } \\
\hline & & & 30 & 60 & 90 & 130 \\
\hline $\begin{array}{l}1-4 \\
1-9\end{array}$ & Off & Ambient & $\begin{array}{l}5.6 \\
8.0\end{array}$ & $\begin{array}{l}7.0 \\
9.2\end{array}$ & $\begin{array}{l}10.6 \\
13.4\end{array}$ & $\begin{array}{r}6.5 \\
10.5\end{array}$ \\
\hline $\begin{array}{l}1-4 \\
1-9\end{array}$ & Off & $370^{\circ} \mathrm{C}$ & $\begin{array}{l}4.8 \\
9.0\end{array}$ & $\begin{array}{l}4.8 \\
7.2\end{array}$ & $\begin{array}{r}7.8 \\
10.2\end{array}$ & $\begin{array}{r}9.8 \\
13.6\end{array}$ \\
\hline $\begin{array}{l}1^{*}-4 \\
1^{*}-9\end{array}$ & Off & $540^{\circ} \mathrm{C}$ & $\begin{array}{l}1.8 \\
4.7\end{array}$ & $\begin{array}{l}1.9 \\
3.8\end{array}$ & $\begin{array}{l}4.2 \\
6.1\end{array}$ & $\begin{array}{r}6.3 \\
11.0\end{array}$ \\
\hline $\begin{array}{l}1-4 \\
1-9\end{array}$ & On & Ambient & $\begin{array}{r}7.7 \\
11.1\end{array}$ & $\begin{array}{l}6.1 \\
9.8\end{array}$ & $\begin{array}{l}12.1 \\
16.6\end{array}$ & $\begin{array}{l}3.7 \\
9.5\end{array}$ \\
\hline
\end{tabular}

Frequency range $=0.178$ to $89.8 \mathrm{kHz}$

Nozzle pressure ratio $=2.8$ 
TABLE 14. OVERALL SOUND PRESSURE LEVEL SUPPRESSION

FOR CONFIGURATIONS 4 AND 9 IN COMPARISON WITH

CONFIGURATION 1.

\begin{tabular}{|c|c|c|c|c|c|c|}
\hline \multirow{2}{*}{ Configuration } & \multirow{2}{*}{$\begin{array}{c}\text { Forward } \\
\text { motion } \\
\text { flow }\end{array}$} & \multirow{2}{*}{$\begin{array}{c}\text { Primary } \\
\text { flow total- } \\
\text { temperature }\end{array}$} & \multicolumn{4}{|c|}{$\triangle$ OASPL at microphone angle (deg) } \\
\hline & & & 30 & 60 & 90 & 130 \\
\hline $\begin{array}{l}1-4 \\
1-9\end{array}$ & Off & Ambient & $\begin{array}{l}6.5 \\
8.8\end{array}$ & $\begin{array}{l}5.5 \\
9.2\end{array}$ & $\begin{array}{l}10.8 \\
15.4\end{array}$ & $\begin{array}{r}7.9 \\
13.9\end{array}$ \\
\hline $\begin{array}{l}1-4 \\
1-9\end{array}$ & Off & $370^{\circ} \mathrm{C}$ & $\begin{array}{r}6.6 \\
10.3\end{array}$ & $\begin{array}{l}5.4 \\
7.9\end{array}$ & $\begin{array}{r}8.0 \\
11.7\end{array}$ & $\begin{array}{r}9.1 \\
19.5\end{array}$ \\
\hline $\begin{array}{l}1^{*}-4 \\
1^{*}-9\end{array}$ & Off & $540^{\circ} \mathrm{C}$ & $\begin{array}{l}2.0 \\
4.1\end{array}$ & $\begin{array}{l}2.0 \\
3.9\end{array}$ & $\begin{array}{l}4.6 \\
7.8\end{array}$ & $\begin{array}{r}5.2 \\
12.2\end{array}$ \\
\hline $\begin{array}{l}1-4 \\
1-9\end{array}$ & On & Ambient & $\begin{array}{r}7.4 \\
10.3\end{array}$ & $\begin{array}{l}5.7 \\
7.9\end{array}$ & $\begin{array}{r}8.8 \\
13.2\end{array}$ & $\begin{array}{l}1.7 \\
7.0\end{array}$ \\
\hline
\end{tabular}

Frequency range $=0.178$ to $89.8 \mathrm{kHz}$

Nozzle pressure ratio $=3.4$

TABLE 15. OVERALL SOUND PRESSURE LEVEL SUPPRESSION

FOR CONFIGURATIONS 4 AND 9 IN COMPARISON WITH

CONFIGURATION 1.

\begin{tabular}{|c|c|c|c|c|c|c|}
\hline \multirow{2}{*}{ Configuration } & \multirow{2}{*}{$\begin{array}{c}\text { Forward } \\
\text { motion } \\
\text { flow }\end{array}$} & \multirow{2}{*}{$\begin{array}{c}\text { Primary } \\
\text { flow total- } \\
\text { temperature }\end{array}$} & \multicolumn{4}{|c|}{$\triangle$ OASPL at microphone angle (deg) } \\
\hline & & & 30 & 60 & 90 & 130 \\
\hline $\begin{array}{l}1-4 \\
1-9\end{array}$ & Off & Ambient & $\begin{array}{l}4.5 \\
6.9\end{array}$ & $\begin{array}{l}3.6 \\
7.7\end{array}$ & $\begin{array}{r}5.2 \\
12.1\end{array}$ & $\begin{array}{r}5.8 \\
15.9\end{array}$ \\
\hline $\begin{array}{l}1-4 \\
1-9\end{array}$ & Off & $370^{\circ} \mathrm{C}$ & $\begin{array}{l}2.3 \\
5.6\end{array}$ & $\begin{array}{l}2.0 \\
5.0\end{array}$ & $\begin{array}{l}4.6 \\
7.8\end{array}$ & $\begin{array}{r}4.7 \\
12.0\end{array}$ \\
\hline $\begin{array}{l}1^{*}-4 \\
1^{*}-9\end{array}$ & Off & $540^{\circ} \mathrm{C}$ & 3.1 & 3.5 & 7.6 & 11.3 \\
\hline $\begin{array}{l}1-4 \\
1-9\end{array}$ & On & Ambient & $\begin{array}{l}4.4 \\
6.3\end{array}$ & $\begin{array}{l}4.6 \\
8.2\end{array}$ & $\begin{array}{r}5.6 \\
11.2\end{array}$ & $\begin{array}{r}5.1 \\
10.8\end{array}$ \\
\hline
\end{tabular}

Frequency range $=0.178$ to $89.8 \mathrm{kHz}$

Nozzle pressure ratio $=4.0$ 
At a pressure ratio of 1.60 (Table 11), the suppression values were smaller than for the 2.20 results. However, the 1.60 results were similar to those obtained by Maestrel1o ${ }^{2}$ at a slightly lower pressure ratio. Since flow over perforated surfaces is known to be a generator of tones 7,8 at a Strouhal number of about 0.2 , there was concern about the porous plug hole diameters of $0.168 \mathrm{~cm}$ resulting in objectionable tones at a model frequency of about $40000 \mathrm{~Hz}$. The plug hole size was selected to be $0.168 \mathrm{~cm}$, the smallest diameter available commercially in perforated stainless steel sheets that were required to withstand hot flow. The test showed the expected tones near $40000 \mathrm{~Hz}$, but only at subsonic jet speeds at pressure ratios of 1.60 and below. The tones were not strong enough at the 1.60 pressure ratio to have significant impact on OASPL's for most cases, but they were sufficiently strong to cause a $15 \mathrm{~dB}$ increase in the $40000 \mathrm{hz}$ one-third octave band over the level that is obtained by fairing a smooth-curve between the 20000 and the $80000 \mathrm{~Hz}$ one-third octave band data points. The tones were absent on Configurations 7 and 8, partially absent on 9, moderately strong on 4-6, and very strong on 10. The tones were most intense at Microphone 13 and became considerably weaker as the observer moved toward Microphone 2. Examples of the effects of the tones on the $1 / 3$ octave band sound pressure levels are shown in Figures A9, A10, A13, A14, A17, A18, $\mathrm{A} 33$, and A34.

Acoustic Shield Effects - An acoustic shield was placed between Configuration 4 and the microphone circle to demonstrate the usefulness of the shield for porous plug jet noise suppression. The shield consisted of a flat plate located $27.9 \mathrm{~cm}$ from the model centerline. The plate began at a point $7.6 \mathrm{~cm}$ downstream of the forward-motion duct exhaust plane $(2.3 \mathrm{~cm}$ downstream of the jet nozzle exhaust plane) and extended a distance of $38.1 \mathrm{~cm}$ parallel to the model centerline in the downstream direction; the lateral extent of the plate (perpendicular to the streamwise direction) was $244 \mathrm{~cm}$. The width of the plate, $38.1 \mathrm{~cm}$, was slightly less than the length of the porous plug, $39.5 \mathrm{~cm}$. Hence, the main acoustic paths from the model to the microphones were around the long edges $(244 \mathrm{~cm}$ ) of the plate. Appendix Figures A65-A68 show sound pressure levels measured with and without the shield in place. 
Significant sound suppressions were recorded for the subsonic nozzle pressure ratio $(1.60)$ but not at the supersonic nozzle pressure ratio $(2.80)$; the reductions were significant only at angles of about $60^{\circ}$ or more from the jet axis (See Figure A66).

The same shield was used with Configuration 10, and the resulting sound pressure levels are shown on Figures A69-A72. The porous plug in this case is $60.6 \mathrm{~cm}$ long, but the shield is still about as effective in suppressing the noise as it was on Configuration 4, indicating that the main noise sources are on the upstream part of the plug, near the shield.

Jet Noise Suppression with Hot Flow - The effect of hot flow on porous plug sound suppressors is illustrated by Figures 26 and 27. Figure 26 shows a comparison of Configuration 1, 4, and 9 with cold flow; Figure 27 shows the same comparison for a flow total temperature of $370^{\circ} \mathrm{C}$. Table 13 shows that with the cold flow, the OASPL is reduced by $13.4 \mathrm{~dB}$ (at Microphone 9) in Configuration 9 as compared to Configuration 1 . The reduction is changed to $10.2 \mathrm{~dB}$ with $370^{\circ} \mathrm{C}$ primary flow, and to $6.1 \mathrm{~dB}$ with $540^{\circ} \mathrm{C}$ primary flow.

The $540^{\circ} \mathrm{C}$ run with the reference nozzle was made without the forward-motion duct in place - i.e., for Configuration $1^{*}$ - under the assumption that the absence of the forward-motion duct would not make a significant difference in the results. However, Table 8 shows that in the case of cold flow, the presence of the duct increased the OASPL by $1.1 \mathrm{~dB}$. Hence, the real reduction in OASPL by Configuration 9 as compared to 1 is probably a little larger than the $6.1 \mathrm{~dB}$ given above.

A structural problem encountered on a1l hot flow runs on Configurations 4 and 9 may have increased the notse levels during these runs. Thermal stresses caused in the bolts holding the porous plug and the forebody between the porous plug and the centerbody struts to loosen and the plug and forebody to droop downward during hot runs. For example, during the $370^{\circ} \mathrm{C}$ run with Configuration 9, the downstream tip of the porous plug was monttored carefully for droop. Little droop was observed during testing at pressure ratios of 1.60 and 2.20 , but during tests at pressure ratios of $2.80,3.40$, and 4.00 , the tip gradually dropped downward by $1.5 \mathrm{~cm}(0.6 \mathrm{ln}$.$) at the end of the last pressure$ 


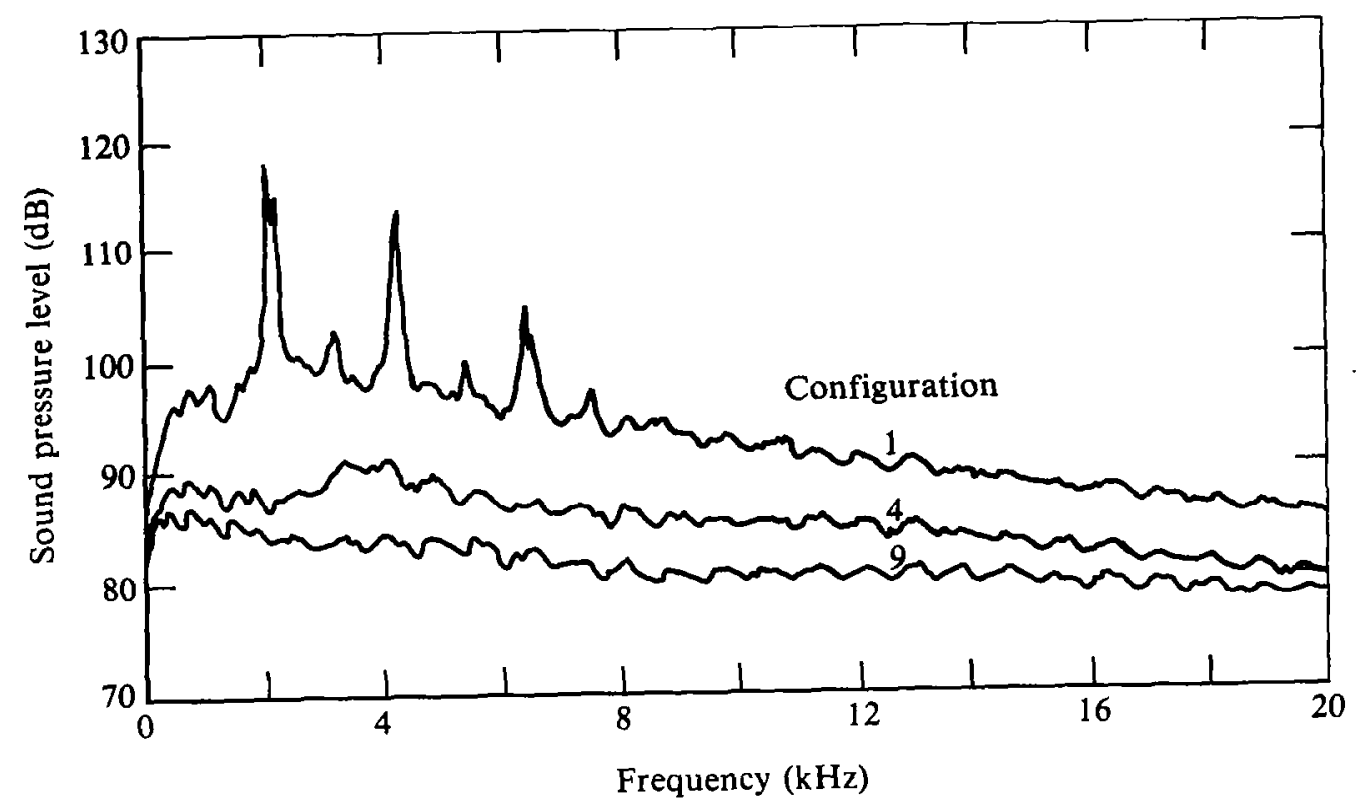

Figure 26. Narrow-band spectra for configurations 1, 4, and 9; microphone 9 , pressure ratio $=2.8$, and bandwidth $=60 \mathrm{~Hz}$. Primary total temperature $=26^{\circ} \mathrm{C}$.

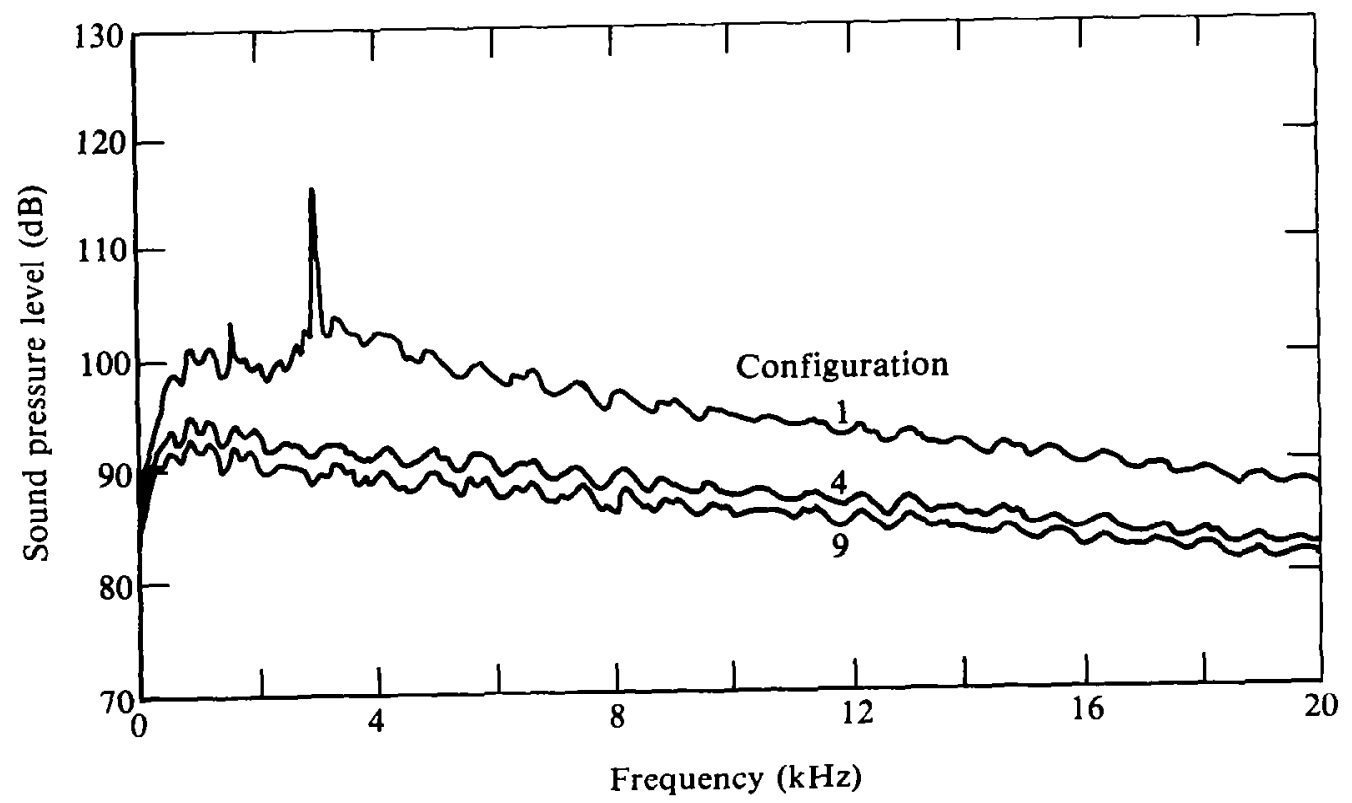

Figure 27. Narrow-band spectra for configurations 1, 4, and 9; microphone 9, pressure ratio $=2.8$, and bandwidth $=60 \mathrm{~Hz}$. Primary total temperature $=370^{\circ} \mathrm{C}$. 
ratio test. This happened in spite of precautions to change the model temperature slowly from cold to hot. Because of the looseness of the porous plugs and forebodies on hot runs of both Configurations 4 and 9, the sound levels generated may have been increased over what they would have been otherwise.

Appendix Figures A37-A52 show one-third octave band spectral data for the hot runs; these can be compared with Figures A9-A12 and A29-A32 for the cold flow runs.

Forward-Motion Flow Effects - Tables 6-10 show that the forwardmotion flow decreased the jet noise at Microphone $9\left(90^{\circ}\right.$ from the jet axis) by about $5 \mathrm{~dB}$ at a pressure ratio of $1.60,3 \mathrm{~dB}$ at a pressure ratio of 2.20 , and $\leqslant 1 \mathrm{~dB}$ at pressure ratios of $2.80,3.40$, and 4.00 . At a pressure ratio of 1.60 , the decrease in overall sound pressure level predicted by the method of Michel and Michalke15 (see Appendix D), is $4.3 \mathrm{~dB}$, close to that measured for each of Configurations 1, 4, and 9 $(5.5,4.5$, and $4.2 \mathrm{~dB}$, respectively). It is interesting that the reductions for both the small diameter and the large diameter porous plug configurations ( 4 and 9) are about the same as for Configuration 1 , which has no plug. Hence, the Michel and Michalke concept of the effect of forward flight on jet turbulence appears to be valid for porous plug flows.

The expected noise reductions from forward flight, as predicted by the Michel and Michalke method, are tied to the variation of the static (without forward motion) sound pressure levels with jet speed, as represented by the velocity exponent m of Equation (D-4). The velocity exponent is near 8.0 for subsonfc jet speeds, but at higher speeds where the jet flow is supersonic, corresponding to pressure ratios above 1.90 , the exponent can be either larger or smaller than 8.0. At the higher speeds the predicted reductions in overall sound pressure levels are generally larger than the $4.3 \mathrm{~dB}$ predicted for a pressure ratio of 1.60 , whereas the reductions measured at Microphone 9 are considerably less than $4.3 \mathrm{~dB}$. The reason for these differences is evidently the effect of the limited forward-motion flow.

The mass flow rate of the forward-motion flow is approximately $10.70 \mathrm{~kg} / \mathrm{s}$. For a main nozzle pressure ratio of 1.60 , the $10.70 \mathrm{~kg} / \mathrm{s}$ is 
about 2.5 times the primary mass-flow rate (see Table 2). For a main nozzle pressure ratio of 2.80 , the forward-motion flow rate is only about 1.5 times the primary flow rate. Since the rate of mass entrainment by the main jet is known to be so large in still air that it entrains its own mass flow rate within a distance of about 3 nozzle diameters downstream of the nozzle, the main jet probably uses all of the available forward-motion flow within a few diameters of the nozzle typically 5 diameters for a pressure ratio of 2.80 . Hence, the 1 imited size of the forward-motion duct is 1ikely to be less than sufficient for obtaining the full effect of forward-motion flow, with the result that the measured sound reductions due to the forward-motion flow are less than predicted on the basis of an infinitely-large forward-motion stream.

As shown in Appendix D, the forward motion predictions depend on the emission angle, $\theta_{0}$. If we use a typical velocity exponent, $m=8.0$, Equations $(D-1)$ and $(D-2)$ show that the effect of $\theta_{0}$ being different from $90^{\circ}$ results in an additional sound reduction of

$$
\triangle \mathrm{OASPL}=(60 \mathrm{~dB}) \log _{10}\left(1+\mathrm{M}_{\mathrm{f}} \cos \theta_{\mathrm{o}}\right)
$$

which amounts to $3.7,2.2$, and $-3.1 \mathrm{~dB}$ for $\theta_{\mathrm{o}}$ equal to 30,60 , and $130^{\circ}$, respectively. The experimental values of the $\theta_{0}$-effect, which can be obtained from Tables 6-10, are generally less than the predicted effect, although the trend with $\theta_{0}$ is in the same direction. This is evidently the result of the limited forward-motion flow.

The one-third octave band spectral effects of forward motion are illustrated in Appendix Figures A53-A64. The Michel and Michalke predictive method indicates that all one-third-octave-band sound-pressure levels of any given spectra should be reduced equally by the forward motion, but the appendix figures indicate that the lower frequency bands were not as strongly affected as the overall sound pressure level. Such a result should be expected when the forward-motion flow is limited, as the lower frequency noise is known to be generated in the first 10 diameters downstream from the nozzle, further downstream than the noise generated at the higher frequencies. Therefore, the entrainment of the 
forward-motion flow will tend to be completed upstream of the region where low-frequency noise is generated, leaving little forward-motion flow in this region.

Porous plug noise suppression values are shown on Tables 11-15, which show that the suppression, calculated by subtracting overall sound pressure levels of Configurations 4 and 9 from that of Configuration 1 , is greater with the forward motion than without the forward motion for pressure ratios of $2.80,3.40$, and 4.00 , and vice versa for pressure ratios of 1.60 and 2.20 . For example, at a pressure ratio of 2.80 and at microphone 9 the overall sound pressure level suppression of Configurations 4 and 9 was 12.2 and $16.6 \mathrm{~dB}$, respectively, compared to 10.6 and $13.4 \mathrm{~dB}$ for cases without the forward-motion effect. At microphone 3 , the same comparisons are 7.7 and $11.1 \mathrm{~dB}$ with forward motion, and 5.6 and $8.0 \mathrm{~dB}$ without forward motion. Hence, the suppression values can change with the forward-motion effect, which indicates that the forwardmotion effect is not generally the same for Configurations 4 and 9 as it is for Configuration 1 .

Tradeoff Between Thrust and Noise Reduction - Possible compromises between thrust efficiency and jet noise suppression are shown in Figures 28-32 for nozzle cold flow pressure ratios of 1.60-4.00. Figure 30 (pressure ratio 2.80 ) shows, as expected, that the thrust coefficients $\mathrm{C}_{\mathrm{T}}$ of Configurations 1 and 2 are about $1 \%$ larger than $\mathrm{C}_{\mathrm{T}}$ for Configuration 3, inasmuch as the struts and plug of Configuration 3 are thrustreducing items - items that must be a part of any real engine with a turbine and its support struts.

In Figure 30, Configurations 4-7 are grouped closely; Configuration 6 shows only a slight thrust reduction compared to Configuration 3 , but Configuration 6 is not quite as quiet as Configuration 5, which has more thrust loss than Configuration 6. Configuration 4 is less efficient than Configuraton 6; Configuration 7 is worse than Configurations 4, 5, or 6. Hence, the extra length of Configuration 7 is liability. The best are Configurations 5 or 6 , depending on the need for a trade between thrust and noise reduction. 


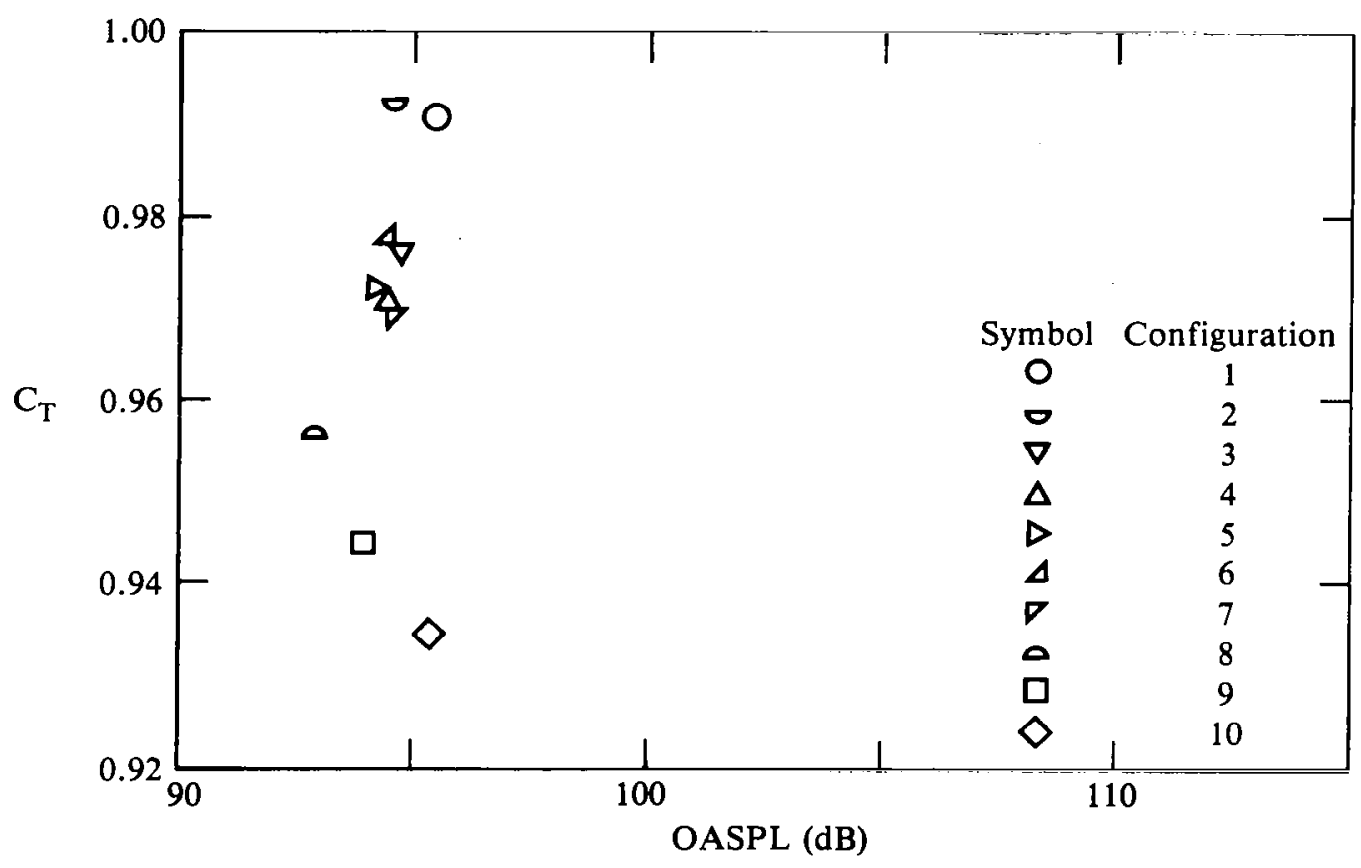

Figure 28. Thrust coefficient as a function of overall sound pressure level; microphone 9 and pressure ratio $=1.6$.

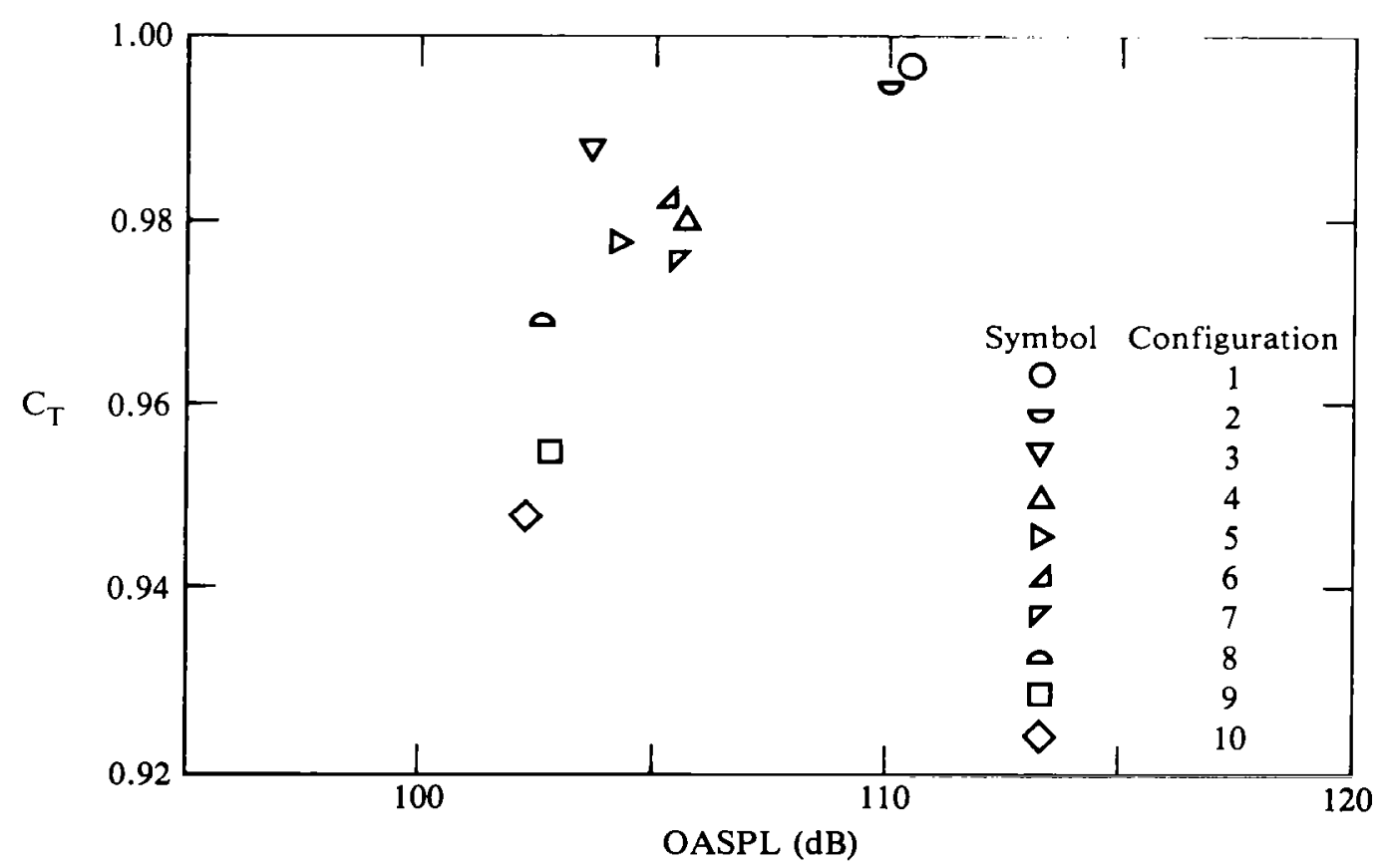

Figure 29. Thrust coefficient as a function of overall sound pressure level; microphone 9, and pressure ratio $=\mathbf{2 . 2}$. 


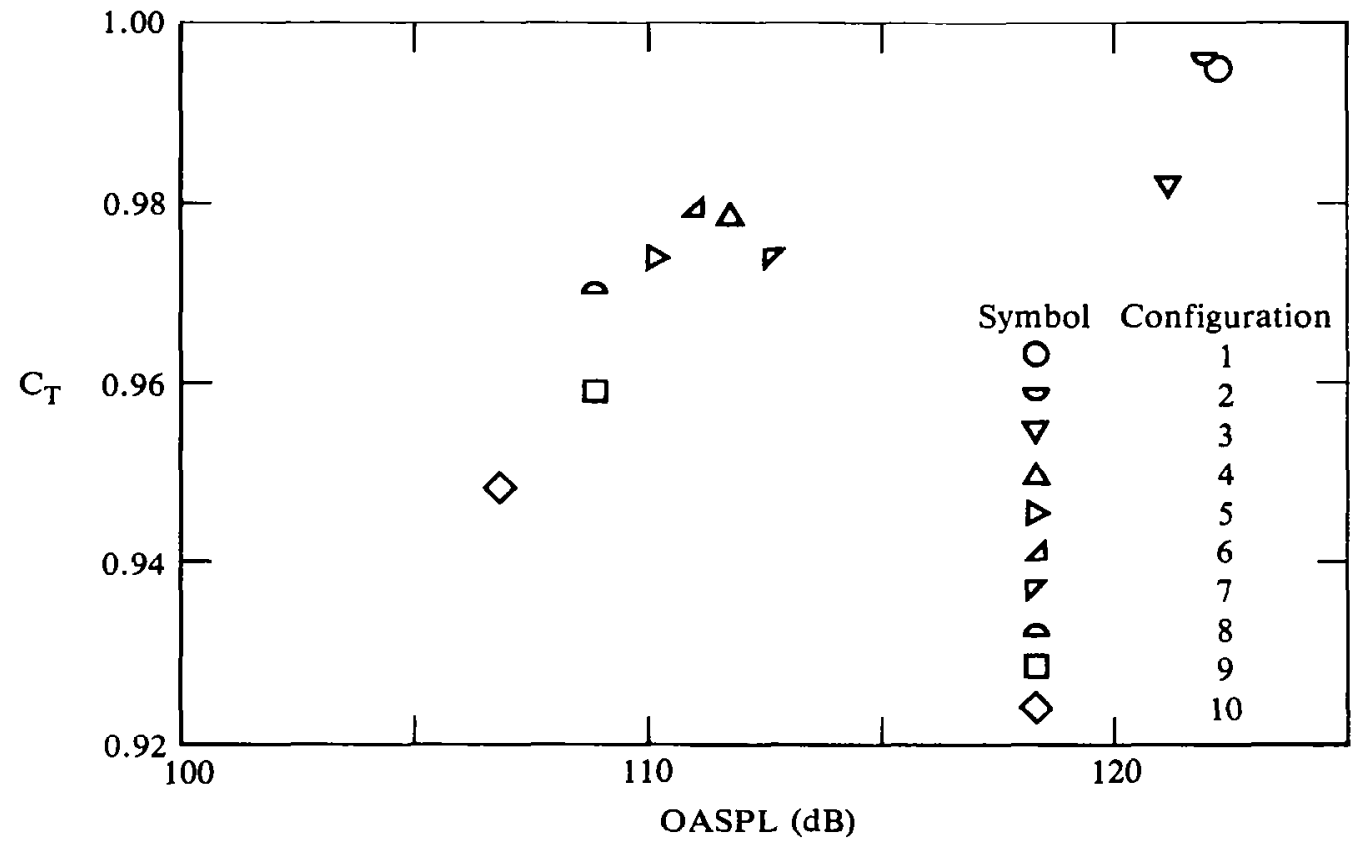

Figure 30. Thrust coefficient as a function of overall sound pressure level; microphone 9 , and pressure ratio $=\mathbf{2 . 8}$.

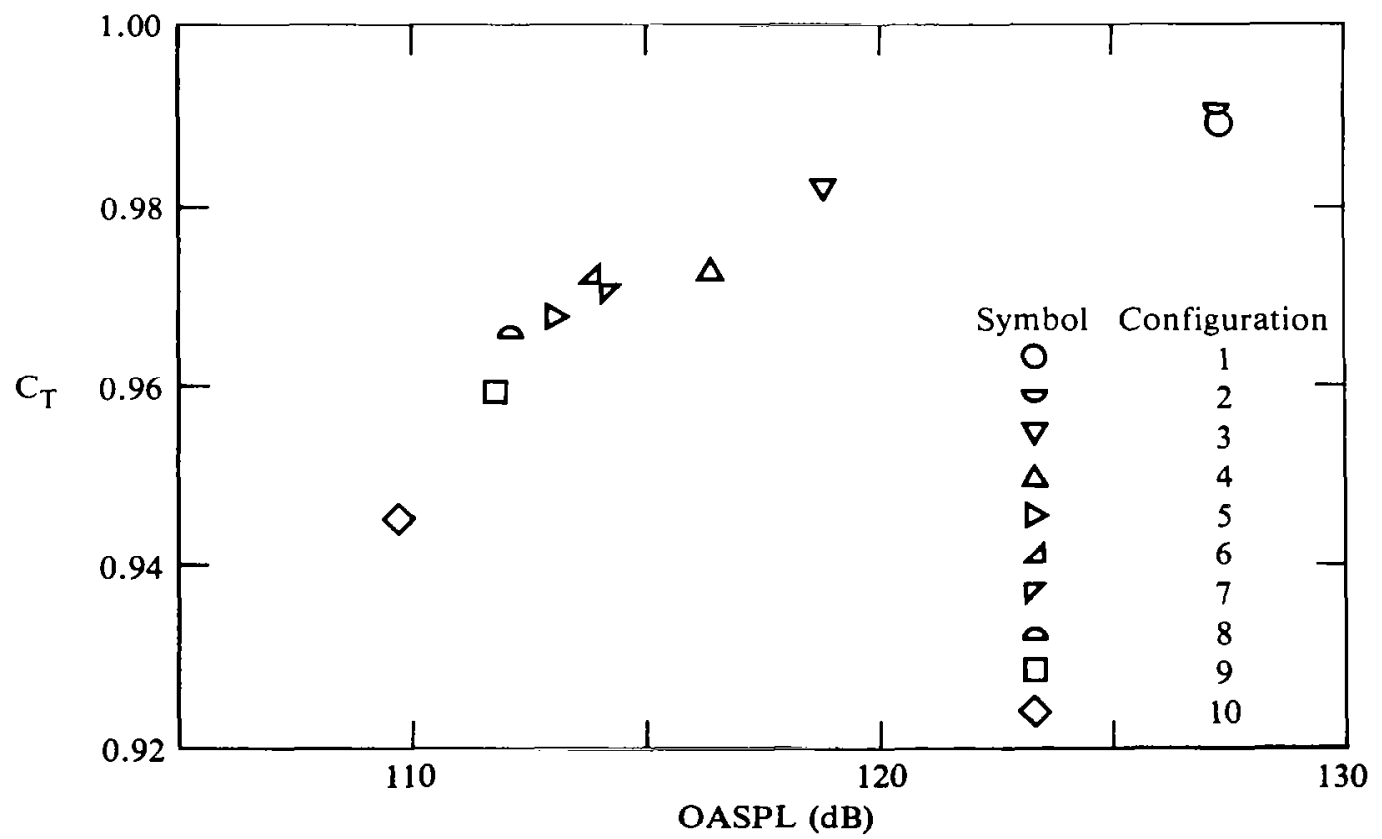

Figure 31. Thrust coefficient as a function of overall sound pressure level; microphone 9 , and pressure ratio $=3.4$. 


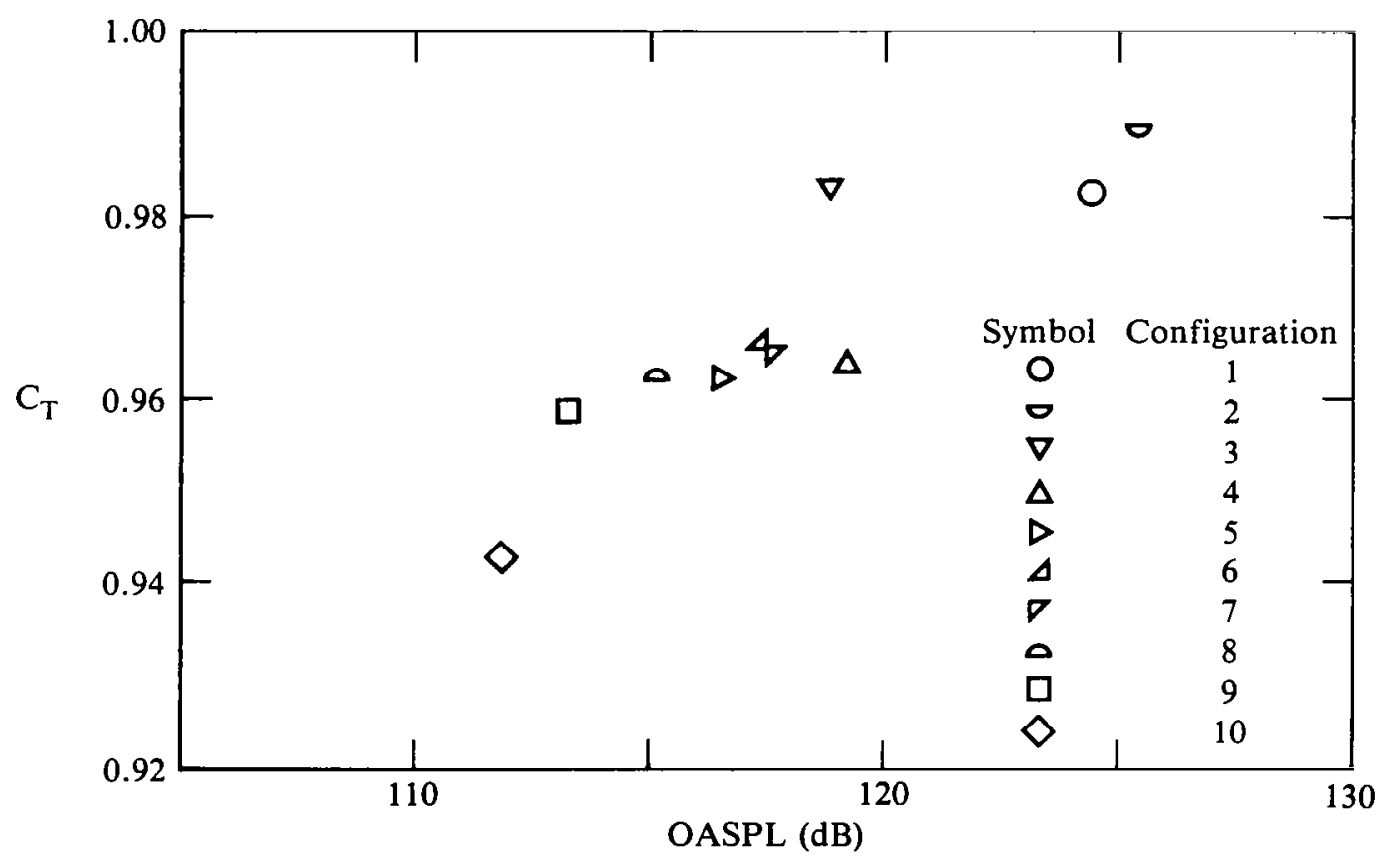

Figure 32. Thrust coefficient as a function of overall sound pressure level; microphone 9 , and pressure ratio $=\mathbf{4 . 0}$.

Configurations 8-10 in Figure 30 have more noise reduction than Configurations 5 or 6 , but Configurations 8-10 also have significantly more thrust loss. Again, a compromise between thrust efficiency and noise reduction would govern a choice among the configurations.

The effect of increasing the porous plug porosity from 2.7 to $5.6 \%$ reduces thrust and noise, as exemplified in Figure 30 both by the comparison of the small radius ratio plugs on Configuration 4 and 5 , and by the comparison of the large radius ratio plugs on Configurations 9 and 10 .

The relative positions of Configurations $1-10$ in Figure 31 and 32 for pressure ratios of 3.40 and 4.00 are much the same as in Figure 30 , pressure ratio 2.80 . The position of Configuration 3 relative to the others is the most mobile, and the thrust difference between Configuration 3 and the most thrust-efficient porous plug, Configuration 6 , becomes larger as the pressure ratio increases to 4.00 .

Figure 29 shows that the relative positions of the 10 configurations are much the same as in Figure 30 for a higher pressure ratio, but 
the jet noise reductions are smaller in Figure 29, and the position of Configuration 3 shows a surprisingly low noise level.

Figure 28 shows smaller noise reductions than the above, evidently because the subsonic jet speed falls to generate any shock noise. Configuration 3 shows slightly less thrust than Configuration 6 , although the plug on Configuration 6 is much larger than the plug on Configuration 3.

Since tradeoffs between the porous plug noise reductions and the thrust efficiency are important, a model for the thrust efficlency has been developed as shown in Appendix $\mathrm{C}$. The model for calculating the thrust coefficient $\left(\mathrm{C}_{\mathrm{T}}\right)$ takes into account the aerodynamic losses caused by boundary-layer friction on the plug and nozzle $\left(\mathrm{C}_{\mathrm{Tf}}\right)$ and the loss caused by flow into and through the holes in the plug $\left(\mathrm{C}_{\mathrm{Th}}\right)$. Losses caused by the plug struts $\left(\mathrm{C}_{\mathrm{Ts}}\right)$ and nozzle flow nonuniformities $\left(\mathrm{C}_{\mathrm{Tn}}\right)$ are also considered. The coefficients needed for evaluating $\mathrm{C}_{\mathrm{Tf}}, \mathrm{C}_{\mathrm{Th}}$, $\mathrm{C}_{\mathrm{Ts}}$, and $\mathrm{C}_{\mathrm{Tn}}$ were adjusted so that the calculated $\mathrm{C}_{\mathrm{T}}$ values closely matched experimental values of $\mathrm{C}_{\mathrm{T}}$. Geometric parameters used in the calculations are given in Table 16, and the calculated $\mathrm{C}_{\mathrm{T}}{ }^{\prime}$ s are given in Table 17.

Since the values of $C_{T}$ for each of the nozzles at the three supersonic pressure ratios of $2.2,2.8$, and 3.4 were approximately equal, the three $C_{\mathrm{T}}$ 's were averaged for each nozzle and were used to evaluate the coefficients, as given in Appendix C. Also, the $\mathrm{C}_{\mathrm{T}}$ values for the pressure ratios of 1.6 and 4.0 are shown in Table 17 . In general, the

TABLE 16. PARAMETERS FOR THRUST EFFICIENCY CALCULATIONS.

\begin{tabular}{lcccccccc}
\hline \hline & \multicolumn{8}{c}{ Configuration } \\
& \multicolumn{1}{c}{$\mathbf{1}$} & $\mathbf{2}$ & $\mathbf{3}$ & $\mathbf{( 4}$ and 6) & $\mathbf{5}$ & $\mathbf{8}$ & $\mathbf{9}$ & $\mathbf{1 0}$ \\
\hline$\ell_{\mathrm{eo}} / \mathrm{d}$ & 0 & 0 & 0.50 & 2.54 & 2.54 & 1.76 & 3.26 & 3.26 \\
$\ell_{\mathrm{e} 1} / \mathrm{d}$ & 0.65 & 0.15 & 0.15 & 0.15 & 0.15 & 0.15 & 0.15 & 0.15 \\
\hline \hline
\end{tabular}


TABLE 17. THRUST COEFFICIENT CALCULATIONS.

\begin{tabular}{|c|c|c|c|c|c|c|c|c|}
\hline & \multicolumn{8}{|c|}{ Configuration } \\
\hline & 1 & 2 & 3 & (4 and 6) & 5 & 8 & 9 & 10 \\
\hline $\mathrm{C}_{\mathrm{Ts}}$ & 0 & 0 & 0.0070 & 0.0070 & 0.0070 & 0.0070 & 0.0070 & 0.0070 \\
\hline $\mathrm{C}_{\mathrm{Tn}}$ & 0.0025 & 0.0050 & 0.0050 & 0.0025 & 0.0025 & 0.0025 & 0.0025 & 0.0025 \\
\hline$C_{T f}$ & 0.0039 & 0.0009 & 0.0029 & 0.0106 & 0.0106 & 0.0156 & 0.0276 & 0.0276 \\
\hline $\mathrm{C}_{\mathrm{Th}}$ & 0 & 0 & 0 & 0.0030 & 0.0062 & 0.0044 & 0.0082 & 0.0170 \\
\hline $\mathrm{C}_{\text {Tcalculated }}$ & 0.9936 & 0.9941 & 0.9851 & 0.9769 & 0.9737 & 0.9705 & 0.9545 & 0.9459 \\
\hline $\begin{array}{l}\mathrm{C}_{\text {Taveraged }} \\
(2.2,2.8,3.4)\end{array}$ & 0.9936 & 0.9938 & 0.9836 & 0.9775 & 0.9730 & 0.9686 & 0.9577 & 0.9472 \\
\hline $\mathrm{C}_{\mathrm{TPR}=1.6}$ & 0.9906 & 0.9918 & 0.9759 & 0.9737 & 0.9736 & 0.9557 & 0.9440 & 0.9344 \\
\hline $\mathrm{C}_{\mathrm{TPR}}=4.0$ & 0.9828 & 0.9898 & 0.9838 & 0.9653 & 0.9628 & 0.9632 & 0.9585 & 0.9427 \\
\hline
\end{tabular}

values of $\mathrm{C}_{\mathrm{T}}$ for 1.6 and 4.0 are lower than at the other pressure ratios, and the nozzle operates more efficient1y at the $2.2,2.8$, and 3.4 pressure ratios.

Table 17 shows the generally good agreement between the calculated and the averaged values. Configurations 4 and 6 are combined because they are similar, and Configuration 7 was omitted because its large plug-length-to-diameter-ratio was expected to result in additional effects (although hindsight indicates that such is not the case). Attention should be focused on the parameters $C_{T f}$ and $C_{T h}$, which are the only ones affected by the addition of a porous plug to the nozzle design of a real engine. (All real engines have struts corresponding to $\mathrm{C}_{\mathrm{Ts}}{ }$ ) Both $\mathrm{C}_{\mathrm{Tf}}$ and $\mathrm{C}_{\mathrm{Th}}$ are increased by increasing plug diameter and by increasing plug length. In addition, $C_{T h}$ is increased directly in proportion to the porosity. Hence, we have the rather obvious conclusion that plug size and porosity should be minimized for thrust efficiency. We would expect $C_{T h}$ to be affected by the actual pressure differentials between the plug interior and the plug exterior. If these differentials are somewhat larger than average, as will be shown later (Figure 46) for Configuration 8 , we would expect $C_{T h}$ to be increased, and vice 
versa, as is the case for Configuration 10 . Such adjustments to $\mathrm{C}_{\mathrm{Th}}$ would tend to bring the calculated $\mathrm{C}_{\mathrm{T}}$ values into better agreement with the measured $\mathrm{C}_{\mathrm{T}}$ values for Configuration 8 and 10 . Hence, a mechanism which tends to reduce the pressure differences will increase thrust efficiency as we11 as noise reduction.

\section{Aerodynanic Test Results}

Although the static pressure probe used for the present investigation has been previously documented, 5,6 tests were conducted in the exhaust of the Mach 1.3 nozzle (Configuration 2) to verify probe performance with respect to spatial resolution and accuracy. Figure 33 is a typical shadowgraph of the reference nozzle flowfield at a pressure ratio of 2.8 . The plume is slightly overexpanded at the nozzle exit,

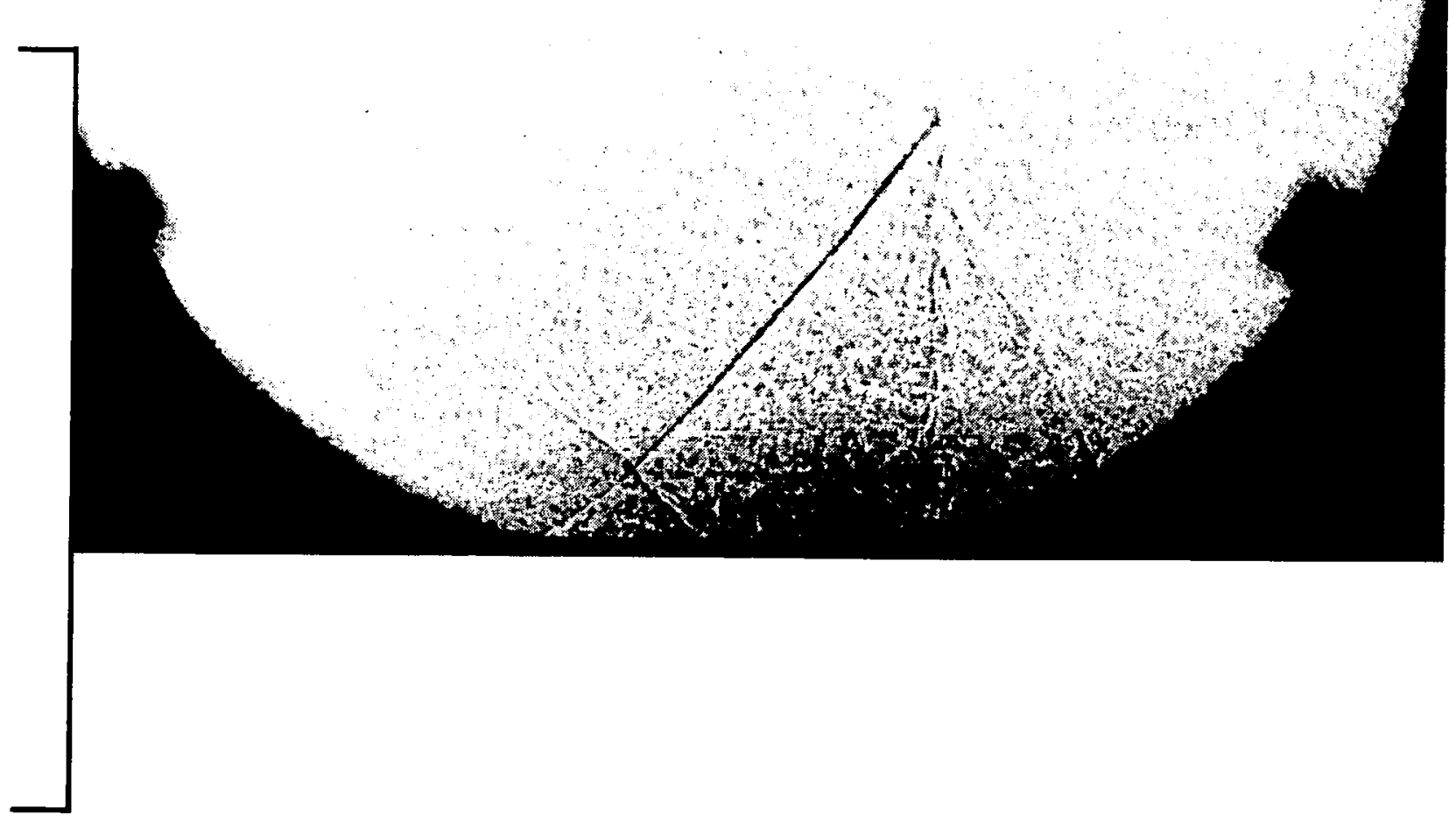

Figure 33. Shadowgraph visualization for reference nozzle at nozzle pressure ratio 2.8 . 
and the early portion of a classical diamond shock pattern is evident. The we11-defined shock structure of the reference nozzle offers a worstcase test of probe resolution, and typical streamwise pressure profiles are shown in Figure 34. The profile obtained at a pressure ratio of 2.8 is consistent with the shock pattern observed in the visualization, and is also consistent with the steep pressure gradients expected in the shock waves. It had been anticipated that the limited spatial resolution of the pressure probe would mask the fine structure of the jet pressure field, but the profiles in Figure 34 demonstrate that the details are sufficiently represented. Shadowgraph movies show that the shock positions are not steady, but that they oscillate with increasing amplitude as the distance from the nozzle exit is increased. Consequently, the mean pressure profiles are distorted because of averaging over a range of shock positions, and the pressure measurements indicate an apparent shock thickness of $5 \mathrm{~mm}$. It is therefore estimated that the static pressure probe is capable of a streamwise resolution of $2 \mathrm{~mm}$, which is adequate tor the present measurements.

Surface pressure measurements, which were obtained using the Configuration 8 instrumented plug, are plotted in Figure 35. The static pressure profiles obtained by traversing the freestream probe $2.5 \mathrm{~mm}$ above the plug surface are plotted for comparison. The trends in both sets of data agree well, further confirming the accuracy of the static probe. Therefore, it was not necessary to instrument any of the other plugs to obtain their surface pressure distributions, because the pressure profiles obtained in the vicinity of the plug surface adequately represent the surface pressure.

Correlation of Pressure and Velocity Measurements - Typical streamwise pressure profiles for the solid plug, Configuration 9T, are illustrated in Figure 36. The profiles were obtained at a radius midway between the plug surface and nozzle 1ip, and for pressure ratios above 1.6 they can be characterized as sinusoids which gradually decay in the streamwise direction. Such results are similar to those obtained by Seiner and Norum in a free jet (see Reference 6, Figure 1), in that they 

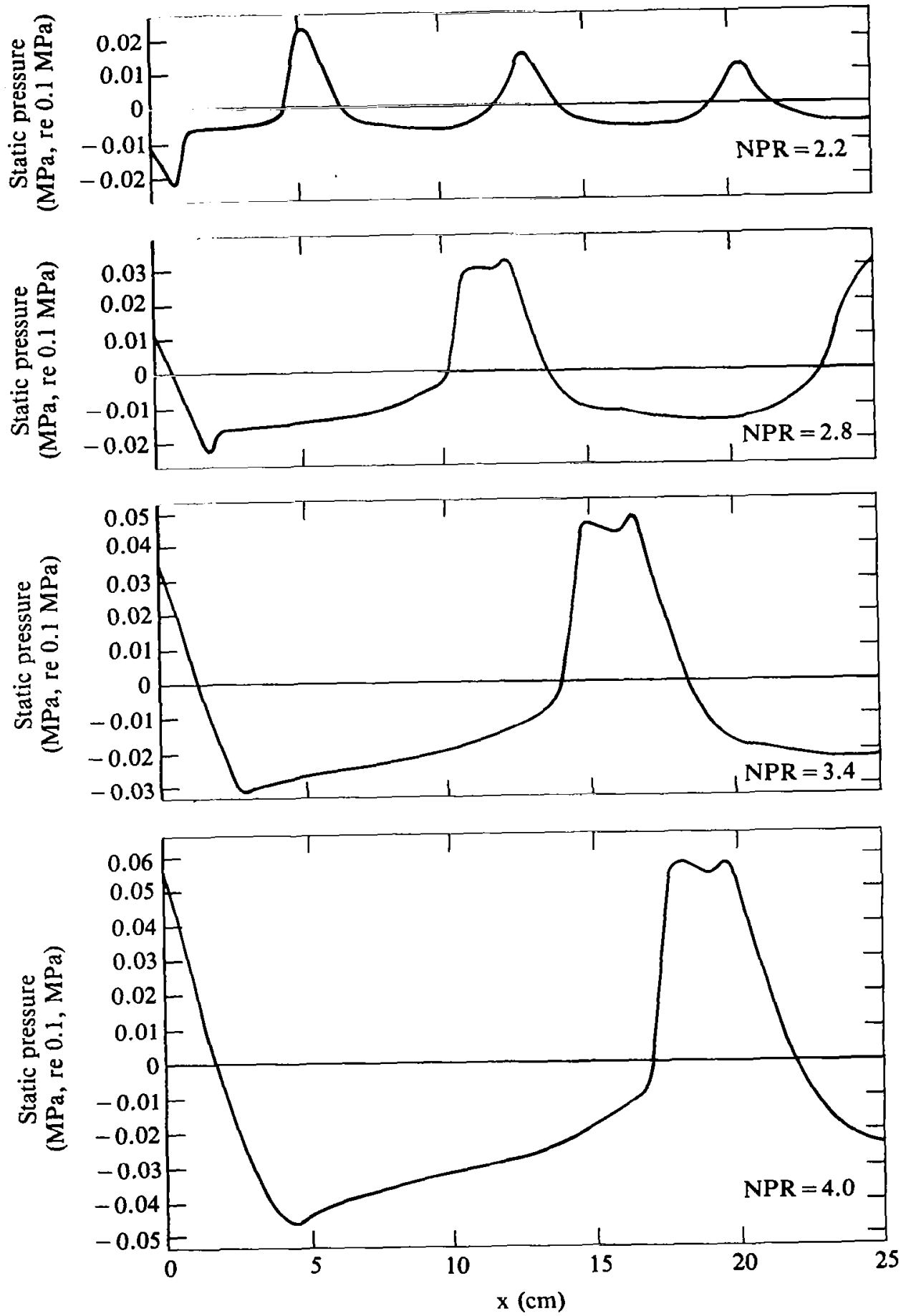

Figure 34. Variation in mean static pressure profiles with pressure ratio for reference Configuration $2(0,0,0)$. 

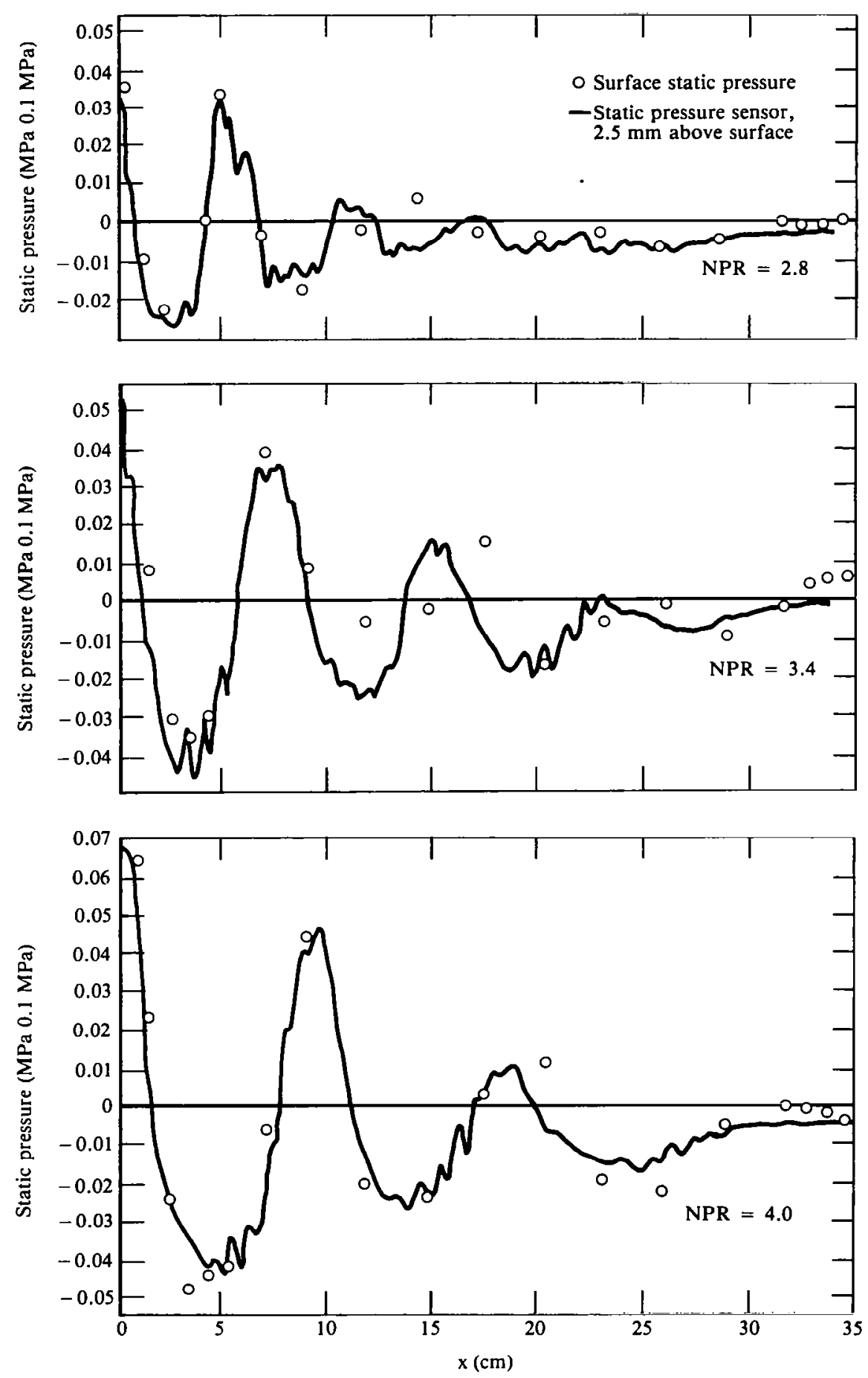

Figure 35. Comparison between surface static pressure and pressure sensed by static probe $2.5 \mathrm{~mm}$ above plug surface for Configuration $8(0.03,0.80,3.7)$. 

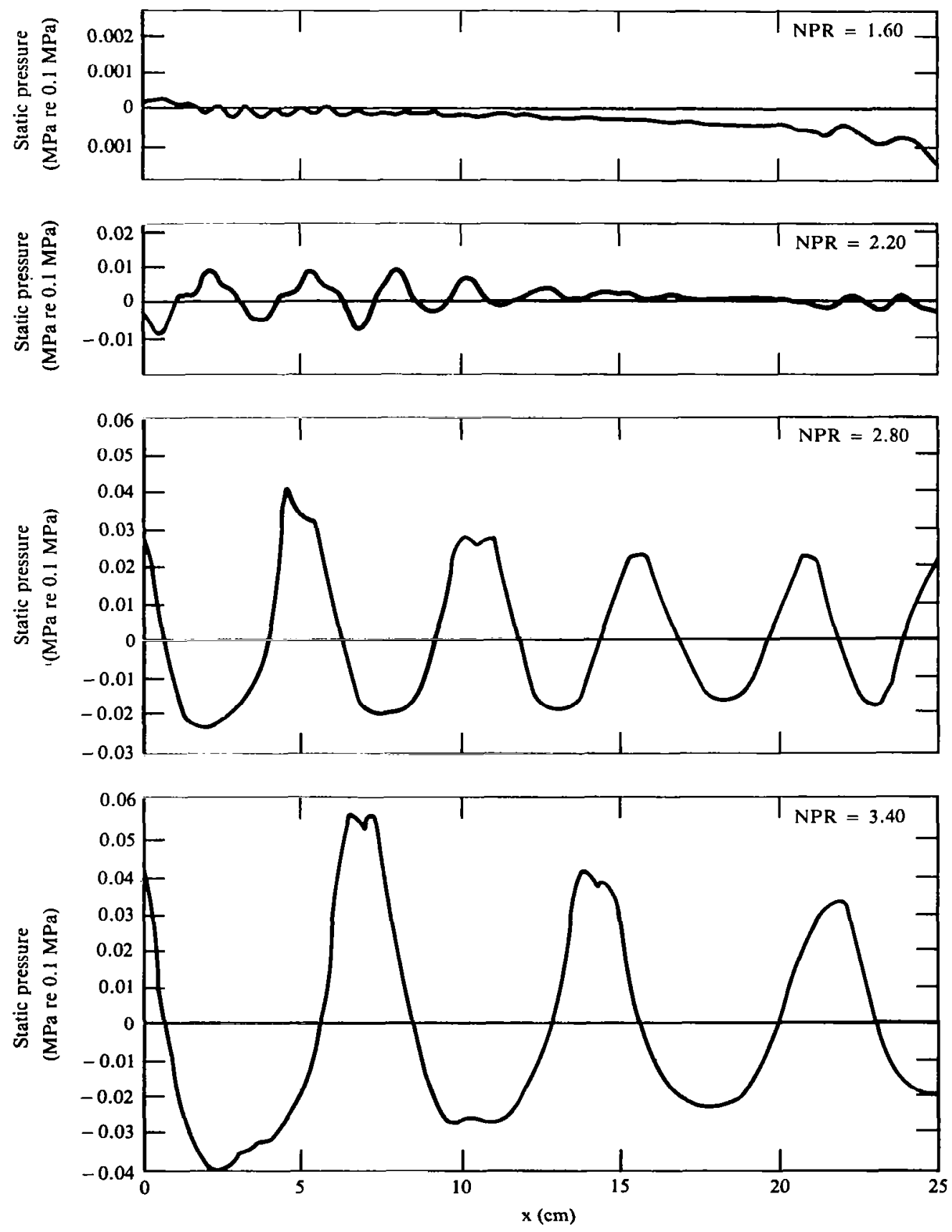

Figure 36. Variation in mean static pressure profiles with pressure ratio for Configuration 9T $(0,0.80,0.52)$. 
indicate a shock cell structure exists for the plug nozzle with zero porosity. As the pressure ratio is increased, the cells lengthen in the streamwise direction, as evidenced by an increase in the measured wavelength. The corresponding mean streamwise velocities measured using the LDV are plotted in Figure 37. The velocities are consistent with the pressure profiles of the preceding figure, and exhlbit similar streamwise periodicities. Velocity profiles calculated from the pressure data using isentropic relations Indicate trends that are virtually identical to those of the measured velocity.

The results demonstrate that the static pressure probe constitutes a valid experimental technique for characterizing plug nozzle flowfields. Consistent static pressure data were obtained using two independent techniques: surface static pressure measurements and LDV velocity measurements. The pressure probe has also been demonstrated to have an adequate spatial resolution for the desired measurements and, as discussed in the section on instrumentation, is therefore well suited to spatial characterization of the jet flowfield. The pressure measurements are used extensively in the present investigation to quantify the development of the plug nozzle flowfield.

Figure 38 is a comparison of the various velocity statistics obtained for the Configuration 8 nozzle at a pressure ratio of 3.4. The profiles are representative of results obtained for other pressure ratios and nozzle configurations, which are presented in detail in the appendix. The mean streamwise velocity, $U$, exhibits a periodicity similar to that observed in the case of Configuration $9 \mathrm{~T}$, although the structure now dissipates after only two cycles. The reduced persistence is in effect of the curvature of the plug surface, which for the shorter Configuration 9 plug begins closer to the nozzle exit. When the plug curvature becomes significant, the streamwise velocity at the radial location of the velocity measurement decreases to subsonic levels, although it is not clear from the limited data whether the cell structure persists closer to the plug surface. 

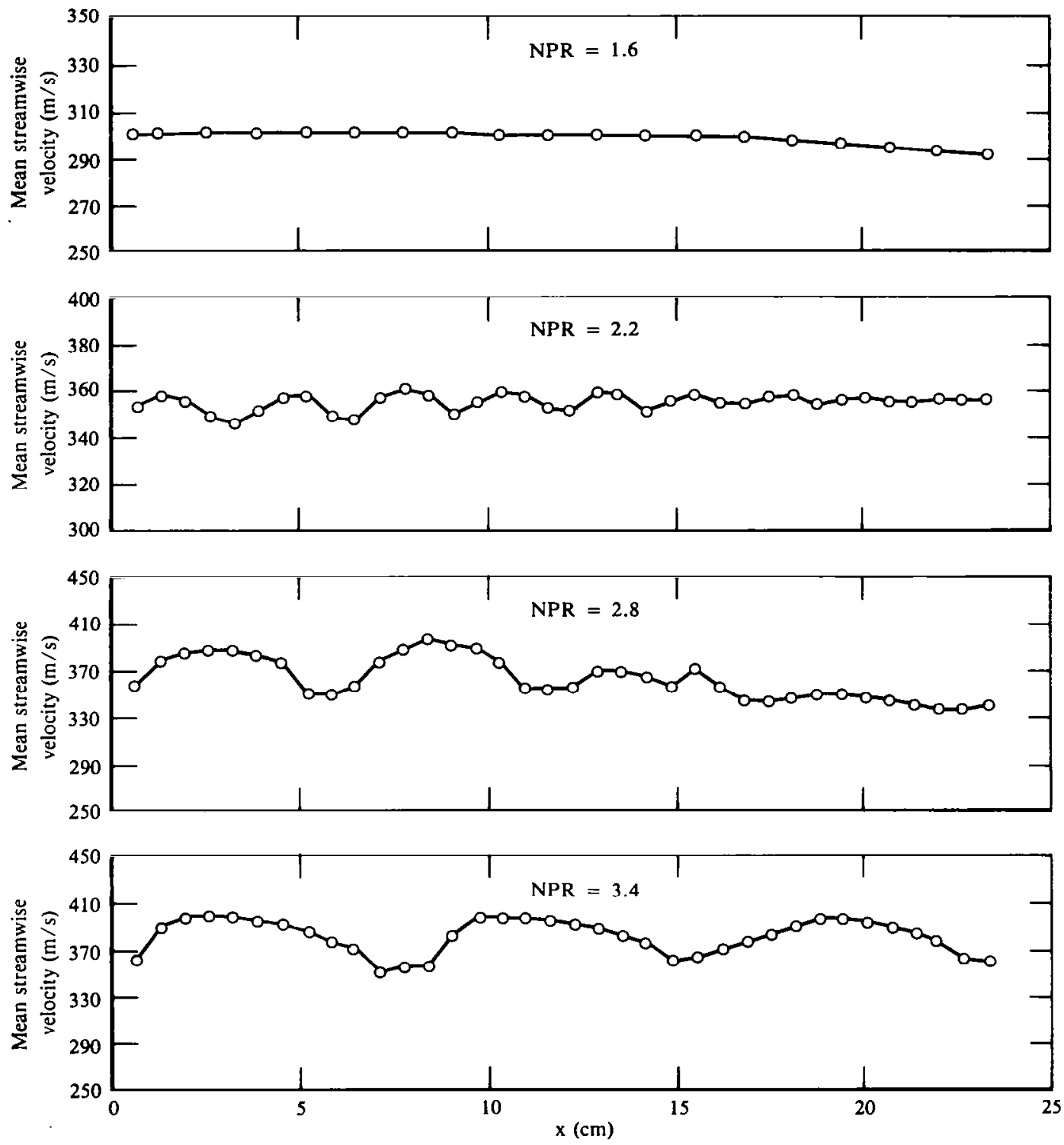

Figure 37. Variation in mean streamwise velocity profiles with pressure ratio for Configuration $9 T(0,0.80,5.2)$. 

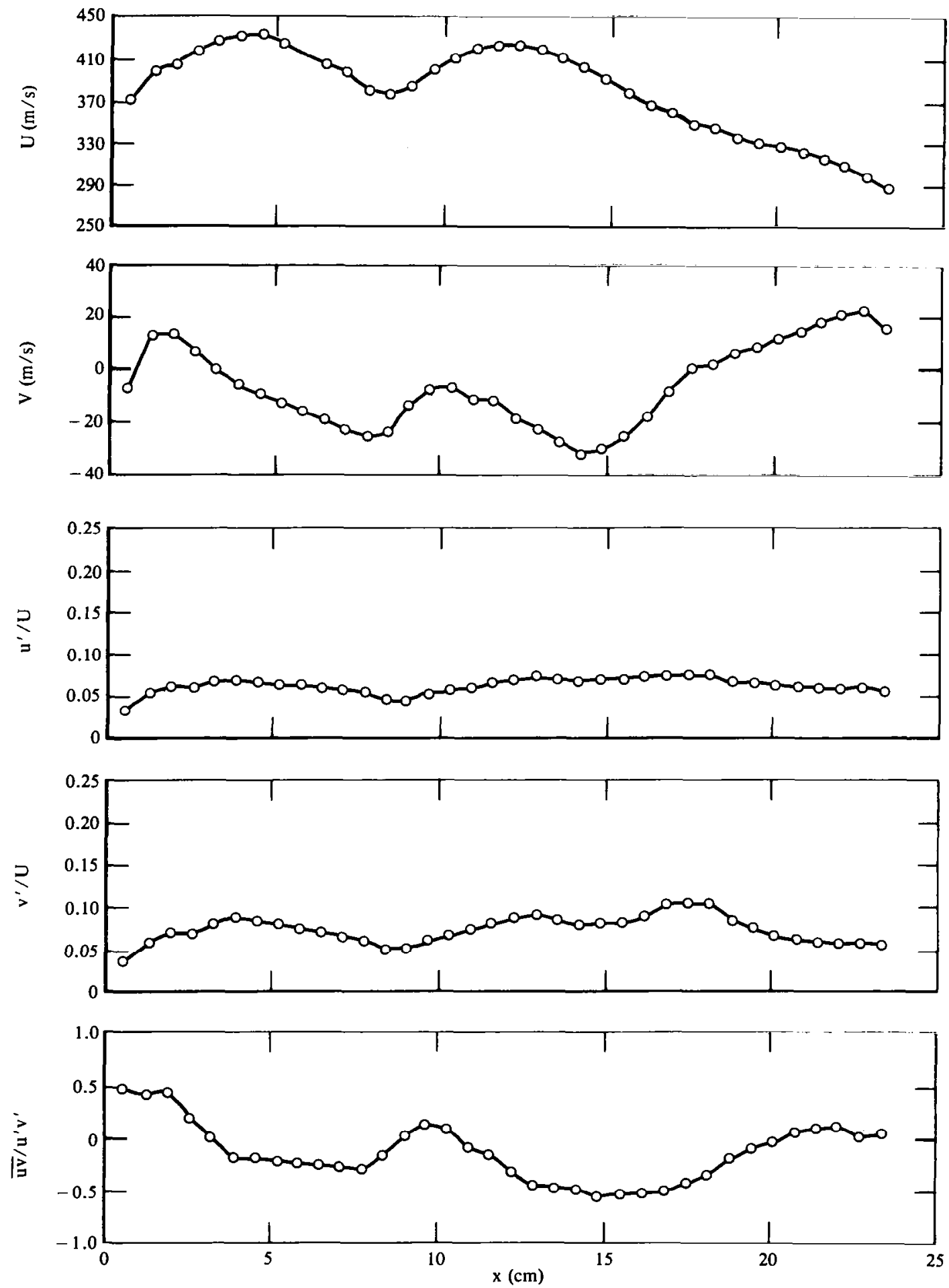

Figure 38. Comparison of velocity statistics for Configuration 8 (0.03, $0.80,3.7$ ) at a nozzle pressure ratio of 3.4 . 
The trends in the other velocity-derived statistical properties generally exhibit the same periodicities as the mean velocity data. The mean radial velocity, $V$, is positive at the beginning of a cycle, and decreases to negative values almost linearly within a cycle. The streamwise and radial turbulence intensities, $u^{\prime} / U$ and $v^{\prime} / U$, have profiles that are similar to those of streamwise velocity in that the intensities are maximum half-way through a cycle. The normalized Reynolds stress, $\overline{\mathrm{uv}} / \mathrm{u}^{\prime} \mathrm{v}^{\prime}$, is large and negative within a cycle and positive or near zero at the beginning and the end. Based on comparisons with the shadowgraph movies obtained under similar flow conditions, a number of mechanisms can explain the characteristics of the varlations in the velocity statistics, as discussed later in this section. The significant point is that the plug nozzle flowfield contained a periodic structure that is similar to the shock cell structure in a free supersonic jet, the existence of which is consistently demonstrated in pressure measurements and velocity statistics.

Summary of Flowfield Measurements - A summary of the flowfield measurements obtained for each plug configuration at a nozzle pressure ratio of 2.8 is depicted in Figures 39-42. In each case, spark shadowgraphs, two-dimensional static pressure distributions, and streamwise and radial velocity profiles are presented. The velocity traverses were obtained in the streamwise direction at a radius corresponding to onehalf the distance between the plug surface and nozzle 11p. The pressure contours were derived from digitized streamwise pressure profiles obtalned at eight radial locations, and were drawn using a contour plotting routine. In each figure, solid and dotted lines represent pressures above and below atmospheric, respectively. The results are the same scale to facilitate comparisons, but care must be exercised because of the limitations of each measurement technique.

The shadowgraph for the reference Mach 1.3 nozzle (Figure 33) illustrates the classical diamond shock structure of an overexpanded jet. A conical shock is evident near the center of the picture, and the static pressure measurements (some of which are shown in Figure 34) indicate that the shock structure is repeated regularly downstream with little decay in shock strength. In contrast, the shadowgraphs for the 

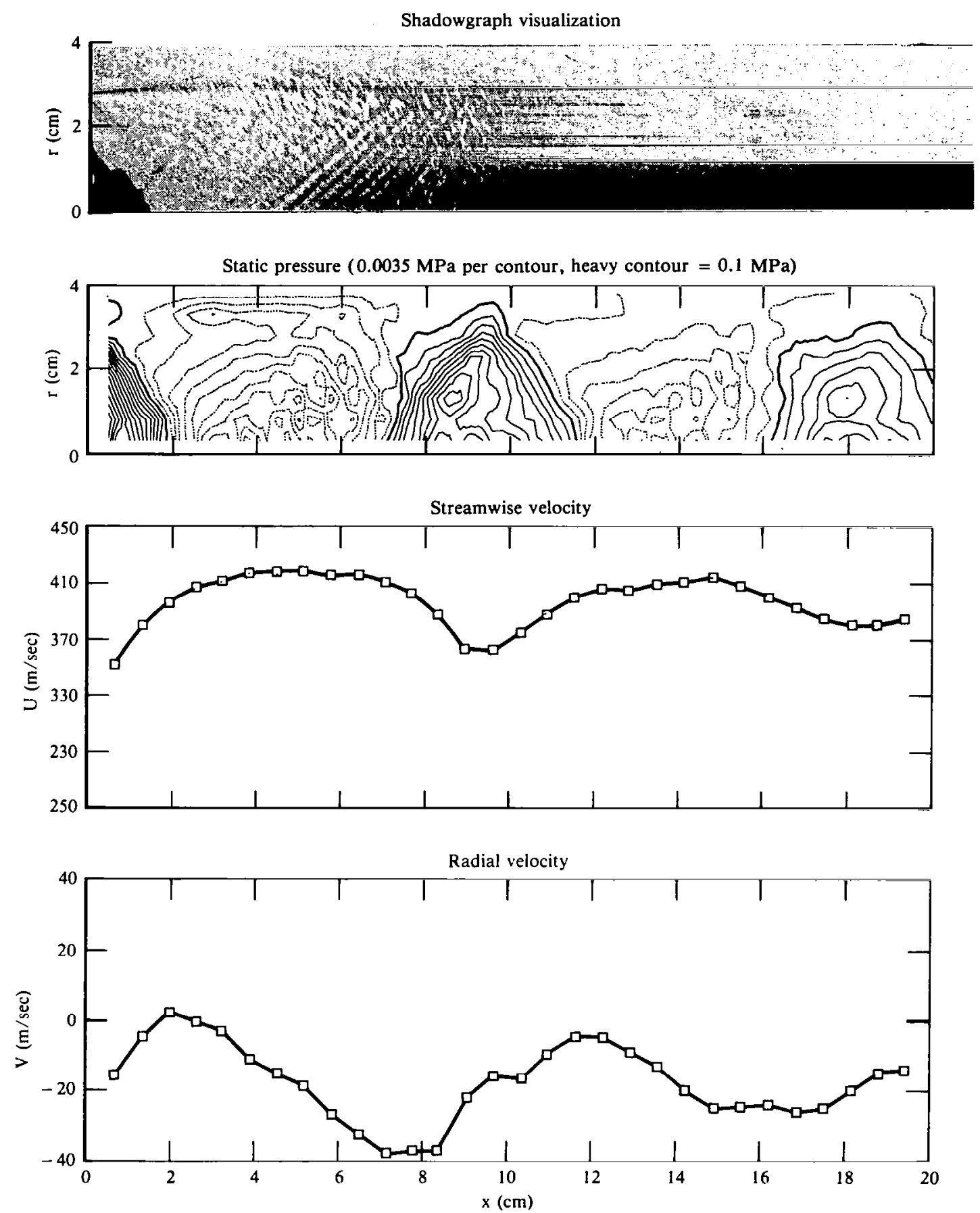

Figure 39. Flow measurement results for Configuration $6(0.03,0.53$, 3.4) at a nozzle pressure ratio of 2.8 . 



Figure 40. Flow measurement results for Configuration $9 T(0,0.80,5.2)$ at a nozzle pressure ratio of 2.8 . 


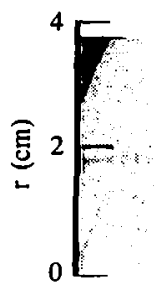

Shadowgraph visualization
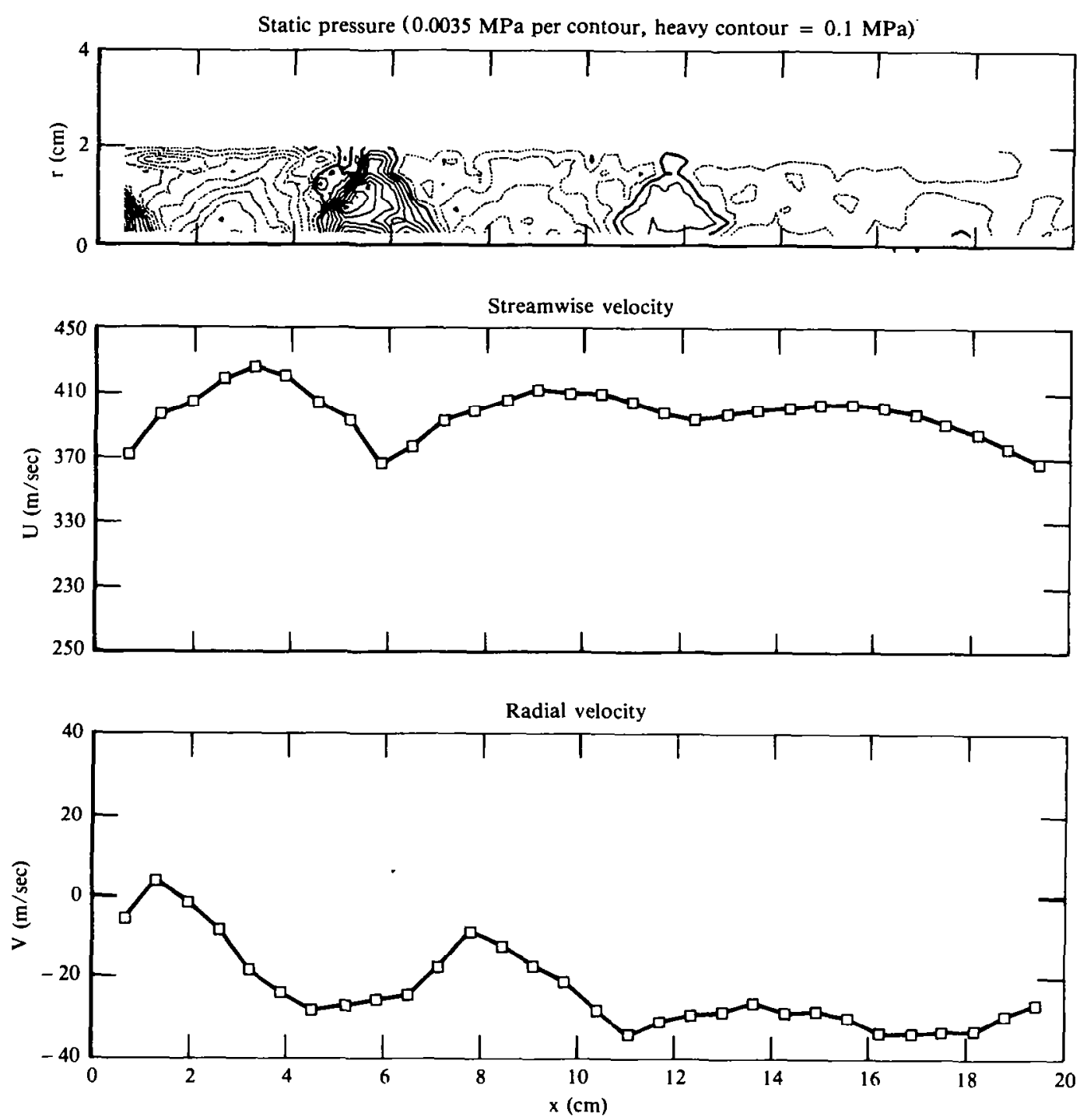

Figure 41. Flow measurement results for Configuration $8(0.03,0.80$, 3.7) at a nozzle pressure ratio of 2.8 . 

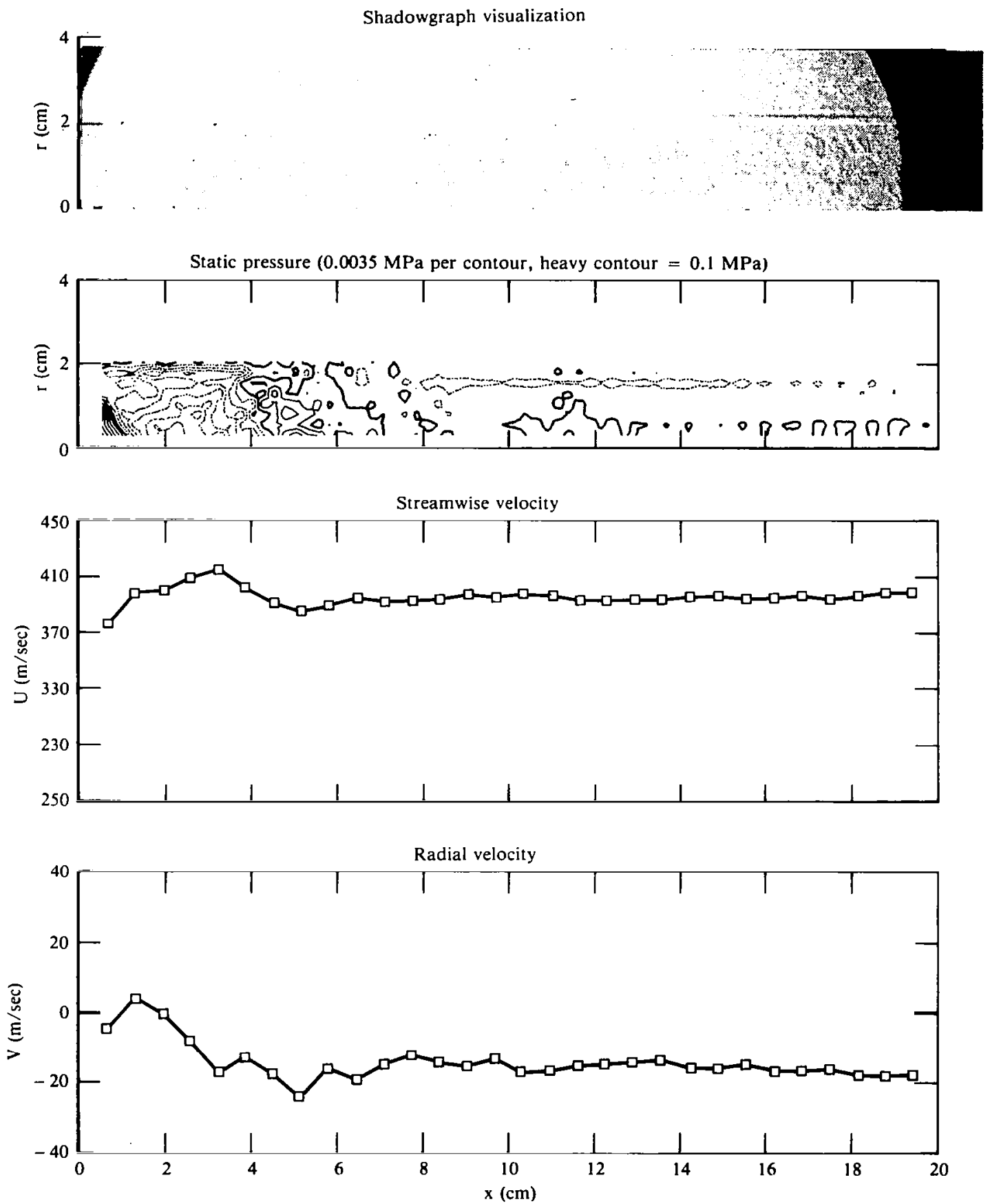

Figure 42. Flow measurement results for Configuration $10(0.06,0.80$, 5.2) at a nozzle pressure ratio of 2.8 . 
various plug nozzle configurations (Figure 39-42) do not show a similar, distinct cell structure. An isolated shock is evident only in the shadowgraph for the solid plug (Figure 40) at approximately $4 \mathrm{~cm}$ from the nozzle exit. Shadowgraphs of the porous plugs (Figures 39, 41, and 42) instead show a series of weak compression waves, which are associated with the holes in the surface of the plug; the significance of this observation is discussed later.

The static pressure distributions indicate a structure that is significantly different from that visible in the shadowgraphs. In Figure 39 the static pressure is presented for the small length-to-diameter ratio plug, Configuration 6 . The simple convergent nozzle has an exit plane pressure that is above ambient, but the pressure decreases below atmospheric after the flow progresses through an expansion emanating from the nozzle lip and its reflection from the plug surface. The static pressure subsequently increases as the flow progresses through a compression and its reflection, but the streamwise pressure gradient is less than that which would occur in a shock At a streamwise distance of between 10 and $12 \mathrm{~cm}$ from the jet exit, another expansion occurs, with subsequent recompression at approximately $16 \mathrm{~cm}$. With the exception of Configuration 10 (Figure 42), which has a high surface porosity, the plug nozzle flowfields contain a series of compressions and expansions that is analogous to the shock trains in a free jet; hence the streamwise periodicity observed in the pressure and velocity profiles. The fundamental difference between the plug nozzle flowfield and the free jet is the reduction of the compression gradients, in accordance with Maestrello's observation ${ }^{2}$ that the plug nozzle flowfield is generally shock free. In the section on Noise Suppression Mechanisms, the fundamental noise reduction mechanism in supersonic plug nozzles is linked to the strength of the compression waves.

The profiles of the mean streamwise and radial velocities constitute independent evidence of the pressure cell structure. The increase in streamwise velocity over the first 3 to $5 \mathrm{~cm}$ from the nozzle exit is consistent with the initial expansion observed in the pressure distributions. The acceleration is followed by deceleration through a compression; the compression is weak and the flow beyond it remains supersonic. 
Depending on the characteristics of the plug surface, the pattern of acceleration and deceleration may be repeated downstream. The radial velocity is outwards after the initial expansion, but the mean velocity vector rotates so that the radial velocity is inwards in the vicinity of the strong compression. The plug surface imposes a constraint on the radial velocity that is accommodated through the compression, but the resulting pressure is typically above atmospheric and the expansioncompression cycle repeats. In the case of the $6 \%$ porosity plug (Figure 42), the direction change through the compression is optimal because the flow downstream is at atmospheric pressure and parallel to the plug surface; hence, the mean and radial velocity profiles are virtually constant beyond approximately $7 \mathrm{~cm}$ from the nozzle exit.

By comparing the shadowgraph movies to the measured velocity data, it appears that the flow between the shock cells is similar to the "shock bottles" in a classical free jet. The rotation of the mean velocity vector is consistent with the curvature observed in the movies at the inside edge of the jet shear layer, although the instantaneous shadowgraphs presented here do not clearly show such structure.

Detailed spatial velocity data were obtained for Configuration 10 at a pressure ratio of 2.8 , and the results are summarized in Figure 43. The velocity measurements were processed in a manner similar to the pressure profiles, although the velocity data were obtained at only four radial locations. Mach number profiles were computed by assuming adiabatic flow. The mean streamise velocity contours indicate an acceleration through an expansion fan that is generally similar to the expansion observed in the pressure plots. The streamwise velocity reaches a maximum just beyond $3 \mathrm{~cm}$ from the nozzle exit and remains constant beyond approximately $6 \mathrm{~cm}$ from the exit plane. The corresponding Mach number contours show that the velocity remains supersonic throughout the core flow region, with the exception of the jet shear layer. The mean radial velocity measurements in the vicinity of the initial expansion indicate flow into the plug over the first centimeter of jet development, in a region where the static pressure is above ambient. Similarly, the next region immediately downstream has a static pressure below ambient, and the mean radial velocity is outwards from the surface 

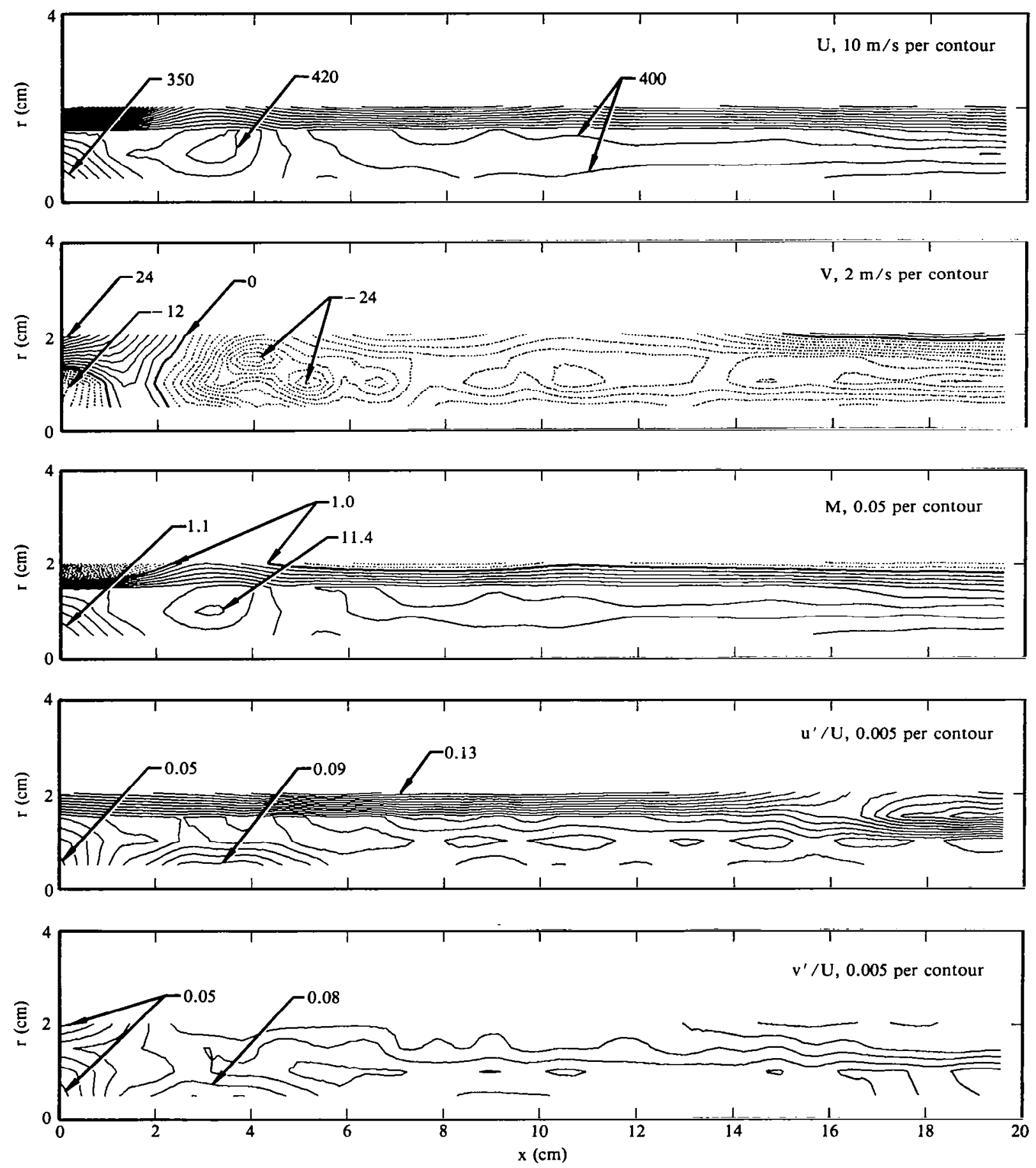

Figure 43. Velocity distributions for Configuration $10(0.06,0.80,5.2)$ at a nozzle pressure ratio of 2.8 . 
of the plug. The contour plots for the fluctuating streamwise and radial velocity components show little spatial variation, with the exception of the streamwise component in the vicinity of the shear layer. The fluctuating streamwise velocity increases rapidly as the thin shear layer is traversed, whereas the fluctuating radial velocity remains small, probably because of a small radial unsteadiness in a region of large velocity gradients.

Scale of Pressure Variations - When the shadowgraphs were compared with the static pressure distributions, there was an apparent discrepancy in the flowfield structure; the difference is a consequence of the characteristics of each measurement technique. Shadowgraphs are sensitive to the second derivative of the density field and emphasize the small-scale structure of the flow. If the large-scale pressure variations that are apparent in the pressure and velocity data also have slowly varying density derivatives, they will not be visible in a shadowgraph. The digitized static pressure profiles present an opportunity to investigate the spatial components of the pressure field. A typical pressure profile is illustrated at the top of Figure 44 . The resolution of the static pressure probe is sufficient to distinguish the sma11-scale variations observed in the shadowgraphs, although the magnitude of the variations is smaller than that of the large-scale pressure changes. Since the smal1- and large-scale pressure variations correspond to short and long streamwise wavelengths, respectively, the two components of the pressure field can be separated by an appropriate digital filter.

A low-pass digital filter was designed with a cutoff frequency corresponding to 1 cycle per $\mathrm{cm}$ and was convolved with the static pressure profile to produce the large-scale pressure variation profile shown in the middle of Figure 44. In addition, the large-scale variations were subtracted from the original profile to produce the effectively high-pass filtered profile shown at the bottom of the same figure. The wavelengths of the small-scale variations correspond to the hole spacing on the surface of the plug and as such represent results similar to the shadowgraphs. In Figure 45, the original and filtered profiles have been combined to determine the two-dimensional pressure distributions 

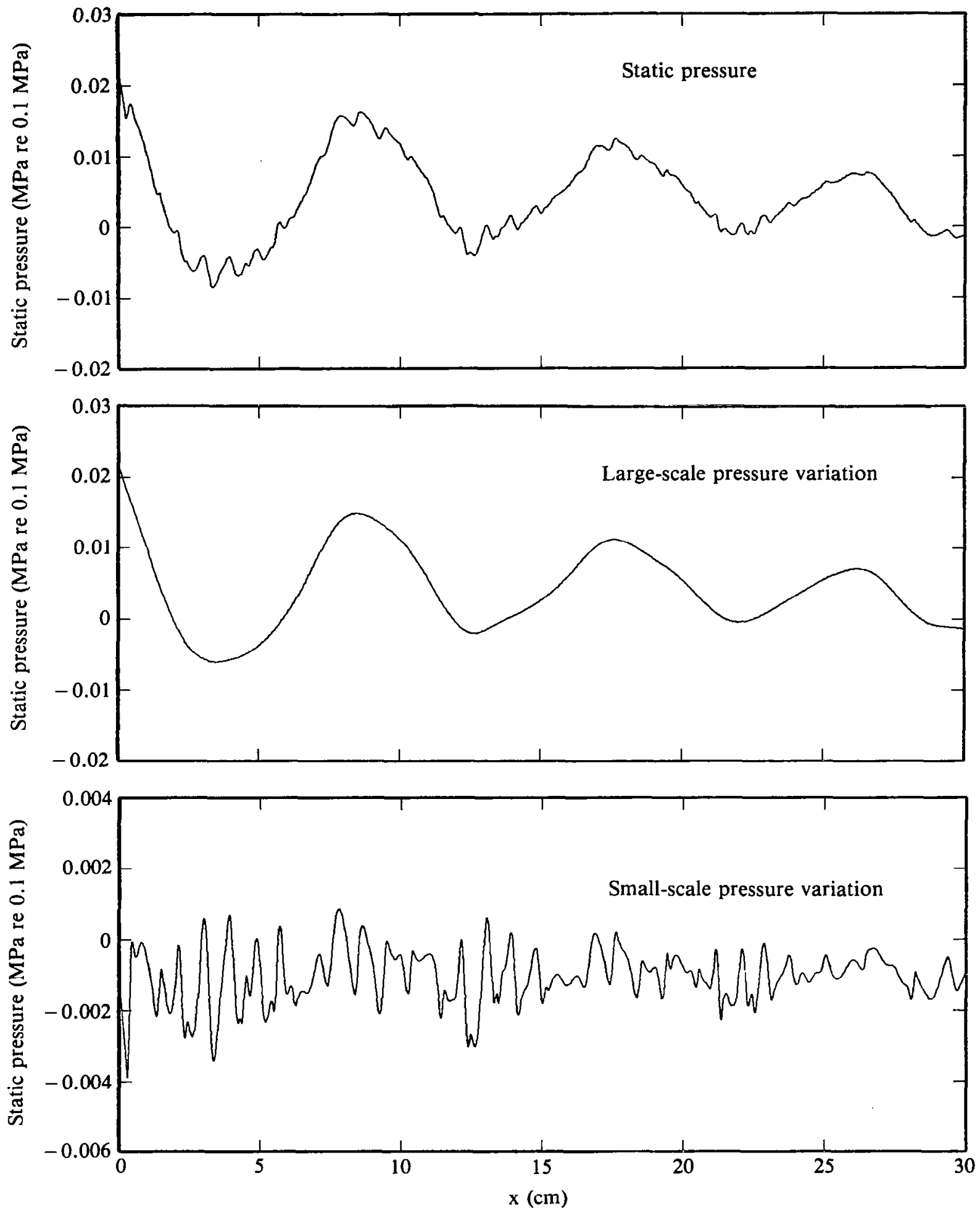

Figure 44. Decomposition of static pressure profiles into large-scale and small-scale variations. 

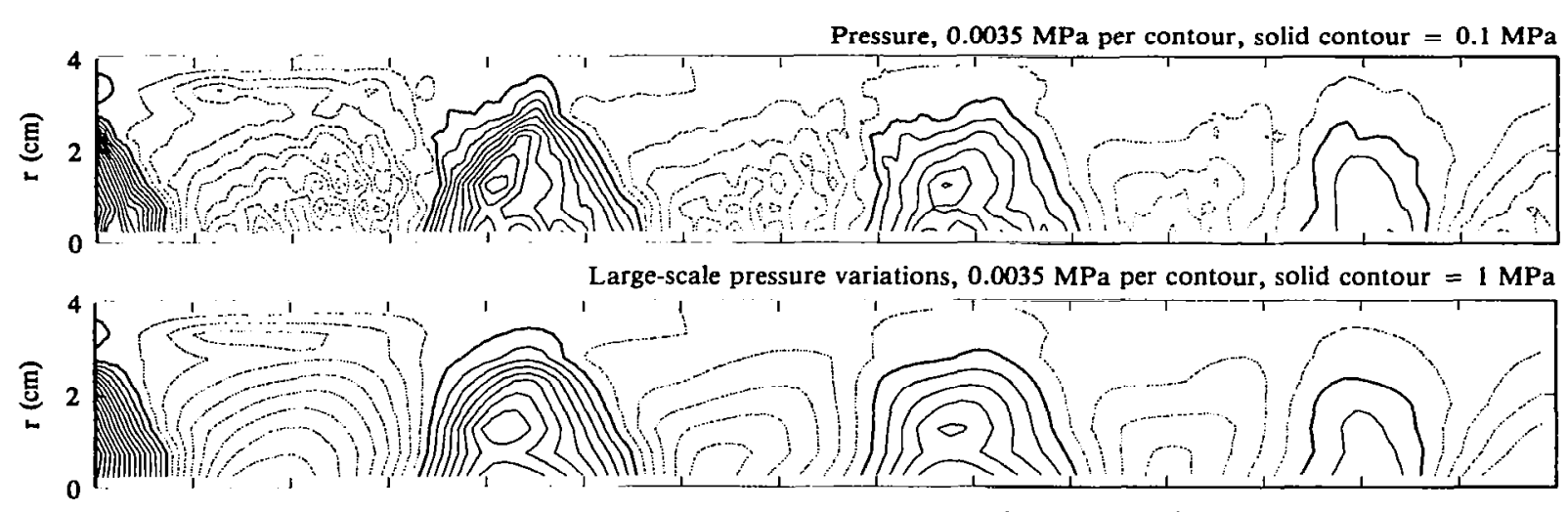

Small-scale pressure variations, $0.0014 \mathrm{MPa}$ per contour

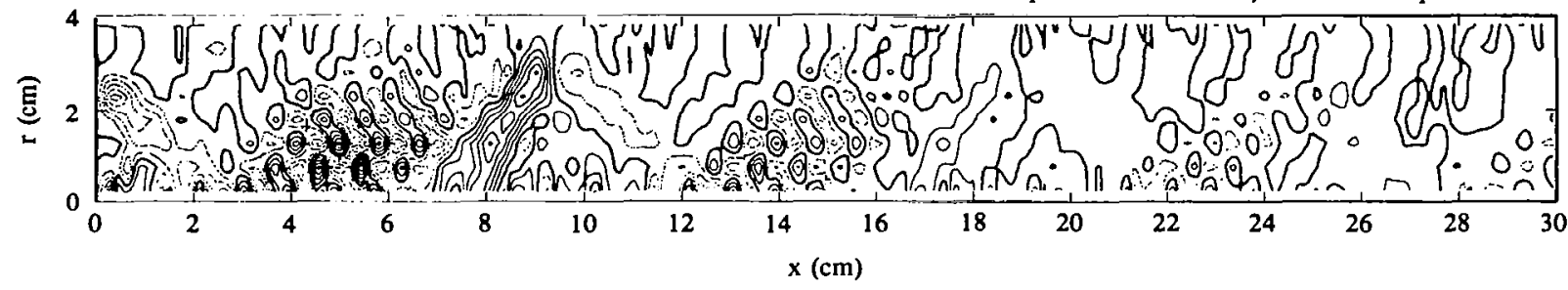

Figure 45. Comparison of static pressure with large-scale and small-scale variations for Configuration $6(0.03,0.53,3.4)$ at a nozzle pressure ratio of 2.8 .

for Configuration 6. It appears that the large scale distributions more clearly represent the streamwise development of the pressure cell structure. The closed contour observed at the nozzle 1ip line approximately 2 to $4 \mathrm{~cm}$ from the nozzle exit is a consequence of shear layer development. Similar contours occur in regions of high static pressure near the plug surface and can be attributed to boundary layer development. The small-scale contours indicate the existence of a compression wave at $8 \mathrm{~cm}$ from nozzle exit and weak shocks from 2 to $6 \mathrm{~cm}$ and from 12 to $16 \mathrm{~cm}$. Because of the limited number of profiles, the contour plotting program is not capable of joining the apparent individual peaks in these regions into distinct lines. Comparisons with the corresponding shadowgraphs show the peaks to be parts of continuous weak shocks. It is apparent that the waves are strongest in regions where the large scale pressure is lowest; such an observation is expected because the low pressure regions also have the greatest streamwise velocity. In the shadowgraphs, there is an apparent concentration of shocklets in the 
vicinity of a compression wave, resulting from the increasing Mach angle as local velocity decreases.

The large-scale pressure distributions for the three largediameter-ratio plug nozzles are compared in the three-dimensional plots of Figure.46. The development of the pressure field is depicted for the first $30 \mathrm{~cm}$ downstream from the nozzle exit. In the case of the solid plug (Configuration 9T), the pressure variations in the cells are large, and the cell pattern is repeated along the length of the plug with little decrease in amplitude. When the surface porosity is increased to $3 \%$ open area (Configuration 8 ), the magnitude of the pressure in the first cell is smaller than that measured for the solid plug, and the peak pressure decays faster with streamwise distance. When the porosity is further increased to $6 \%$ (Configuration 10), the magnitude of the pressure variation in the first cell is even smaller, and there is no evidence of cells further downstream. If these results are compared with the far-field sound pressure levels (Figure 30), a similar trend can be observed in decreasing noise levels. The degree of sound suppression effected by the large diameter ratio plug nozzles is directly related to the strength and persistence of the large-scale pressure cells.

The corresponding small-scale pressure variations for the three large diameter ratio plugs are depicted in contour plots in Figure 47. As expected, these results are similar to the shadowgraphs obtained for the corresponding flow conditions (see Figures 40-42). In the case of Configuration $9 \mathrm{~T}$, the compressions occur in a regular sequence along the length of the plug, with the first two compressions having a shock-like characteristic. The distortion in subsequent cells can be explained by considering the shadowgraph movies which indicate that the third cell experiences an unsteady motion of approximately $1 \mathrm{~cm}$, and the unsteadiness increases further downstream. In the case of the porous plugs, the initial compression does not occur through a single shock, but instead through a series of two or three smaller shocklets originating at the surface pores. Therefore the gradual compression that appears to occur in the large-scale pressure field is actually a sequence of weaker distinct shock waves. In the case of the Configuration 10 plug, the weak 


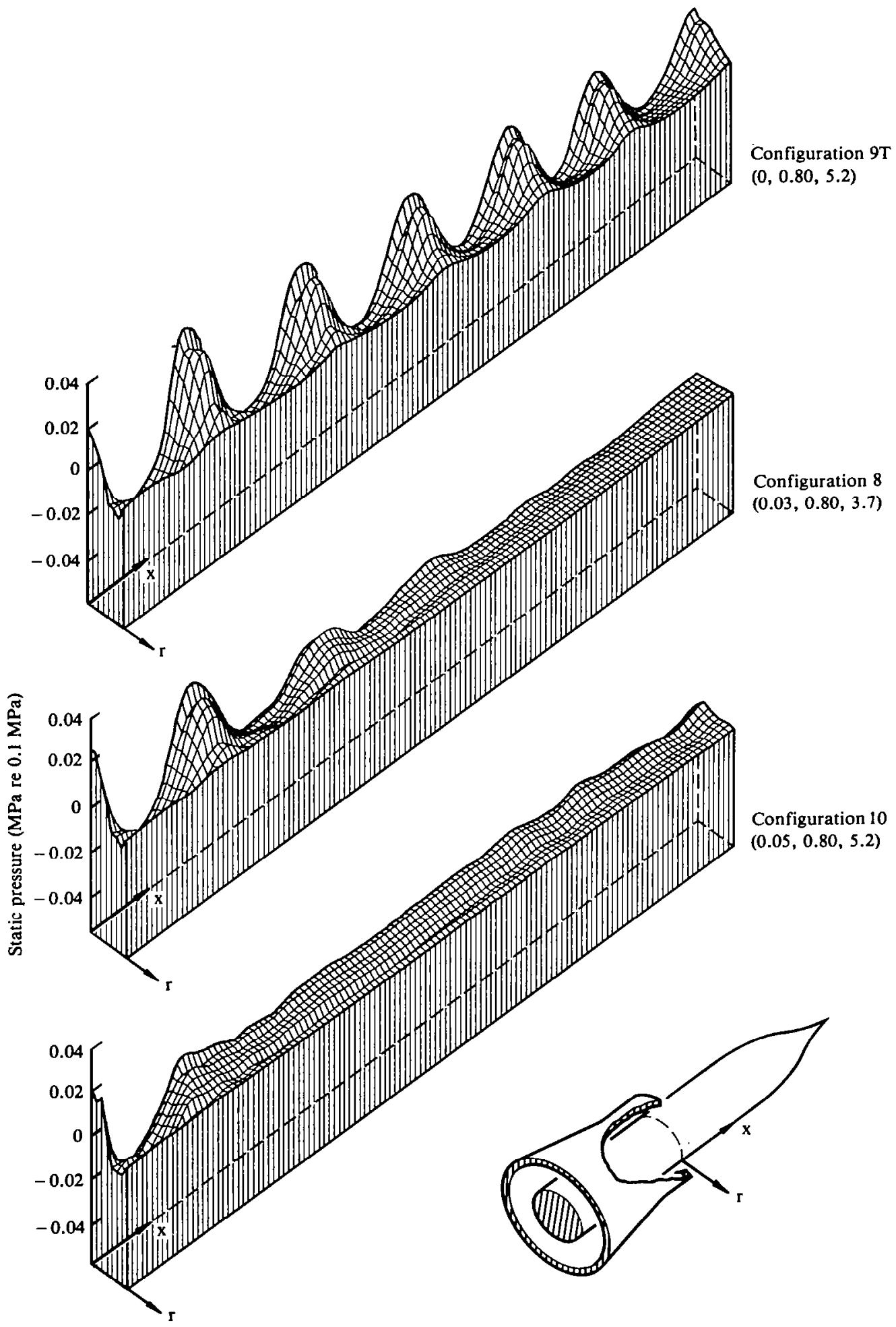

Figure 46. Comparison of plug nozzle static pressure distributions at a nozzle pressure ratio of 2.8 . 

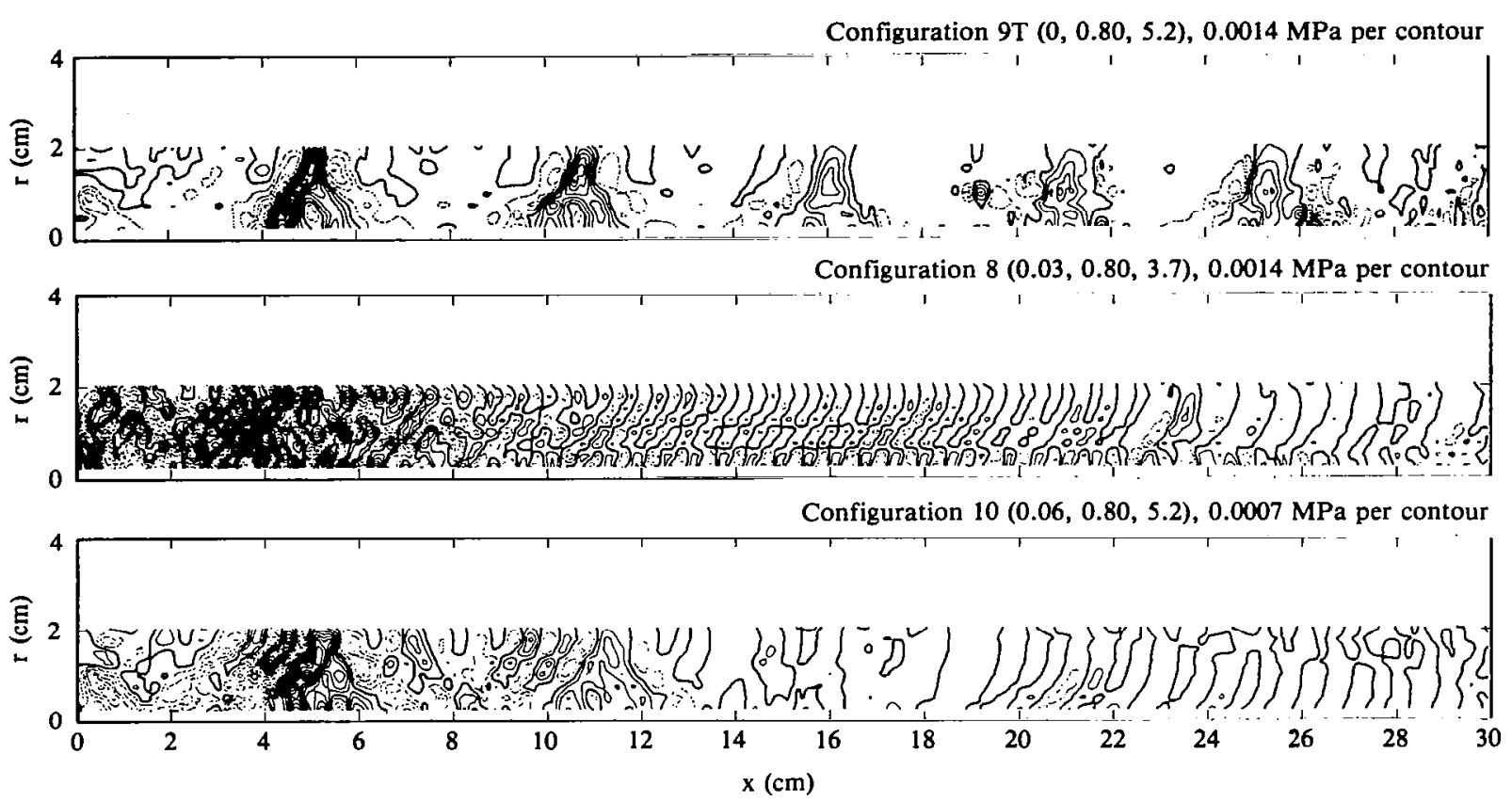

Figure 47. Comparison of plug nozzle small-scale variations at a nozzle pressure ratio of 2.8 .

waves form along the entire length of the plug, but their strength is not as great as in the initial compression; in fact, they are little more than Mach waves that occur as a consequence of the interaction between the plug surface and the supersonic jet flow.

Near-Field Acoustic Results - The results of the near-field acoustic measurements obtained in conjunction with the aerodynamic tests are summarized in Figure 48; they exhibit trends similar to those observed in the acoustic test results. At supersonic velocities there is a consistent 5 to $6 \mathrm{~dB}$ variation in the sound level among the configurations, nearly independently of pressure ratio. The variation is therefore linked to the physical characteristics of the plugs: increasing the surface porosity or plug to nozzle diameter-ratio results in significant noise reduction. From the limited subsonic data, it appears that the surface porosity is not important at low speeds. The variation in sound suppression at a fixed pressure ratio indicates that further modifications to the plug geometry should result in better sound suppression characteristics with even less thrust loss. 


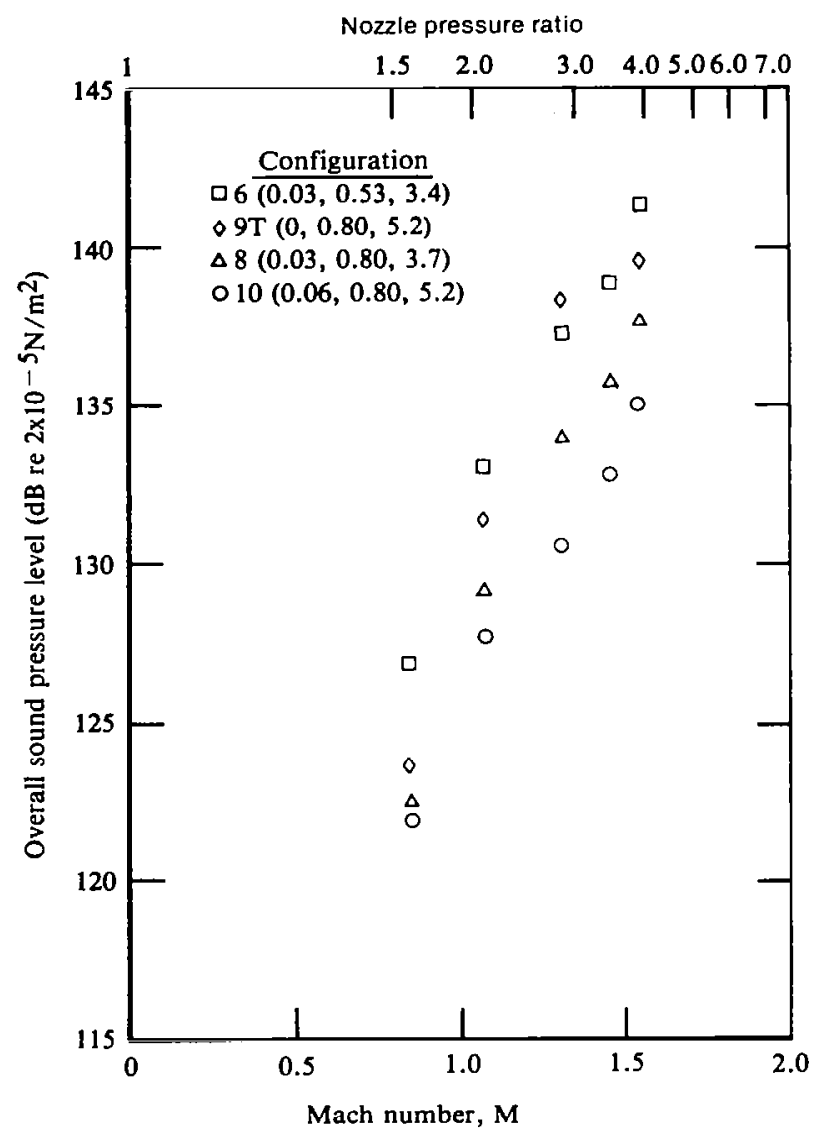

Figure 48. Near-field overall sound pressure level measurements as a function of Mach number for plug nozzles. 


\section{MECHANISMS OF NOISE SUPPRESSION}

To be able to model the sound suppression mechanisms in a plug nozzle flowfield, it is necessary to correlate the acoustic measurements with the aerodynamic features of the flow. The sound generation mechanisms in supersonic free jets have been studied extensively, and it is necessary to determine whether similar mechanisms are responsible for noise generation in plug nozzle jets. Seiner and Norum ${ }^{6}$ conducted an investigation of noise generation in a supersonic plume and determined that the predominant noise source is linked to the interaction between the shock structure and the turbulent mixing layer. A number of theoretical methods have been developed to address the shock/shear-layer interaction mechanism (Lighthil1 ${ }^{9}$; Howe and Ffowcs-Williams ${ }^{10}$ ), but it is more reasonable to establish first the characteristics of the plug nozzle flowfield before attempting to apply similar models to $i t$.

Based on the results of the current tests, it has been established that the plug porosity and the plug-to-nozzle diameter-ratio play important roles in the noise suppression efficiency of the plug nozzle. Although a free jet has no equivalent to surface porosity, the role of plug-to-nozzle diameter-ratio can be further examined by treating the free jet as a limiting case (that is, zero plug diameter) of the plug nozzle. Seiner and Norum determined that the shock cell length in a free jet scales with the nozzle exit diameter and the fully expanded Mach number. For the plug nozzle, the pertinent length scale is the radial height of the nozzle exit, $h$, if the nozzle exit is nominally two-dimensional (as opposed to axisymmetric). In Figure 49, the streamwise length of the first pressure cell normalized with exit height is plotted as a function of the jet exit Mach number, and the data for the various plug geometries exhibit a similar linear Mach number dependence. Because of the axisymmetric geometry of the free jet, however, the cell length data lie on a different curve. It is expected that results for small plug-to-nozzle diameter-ratios would fall somewhere between the two limiting cases, although no such geometries were included in the current test program.

The length of the pressure cells also varies with distance along the plug, as indicated in Figure 50. The normalized cell length is 


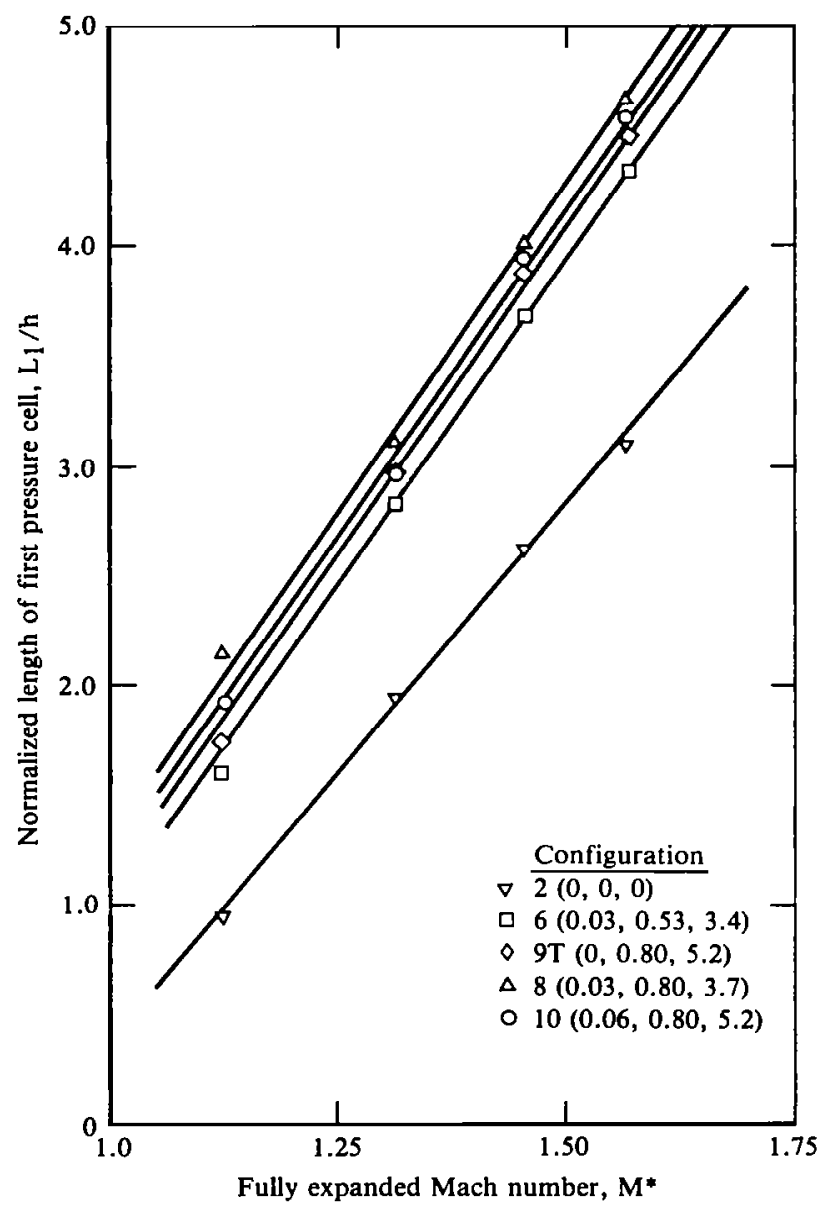

Figure 49. Variation in length of first pressure cell with increasing Mach number.

plotted as a function of cell number; although the solid plug produces more identifiable cells than the plugs with surface pores, the decrease of cell length with cell number is simflar for all nozzle configurations. Seiner and Norum ${ }^{6}$ document a similar phenomenon in a free jet and associate it with the formation of a Mach disk at the center of the jet. Although the formation of an annular equivalent (such as a Mach ring) is possible, the pressure field and shadowgraph results present no evidence of such structures in the plug nozzle flowfield; instead, the decrease in cell spacing can be attributed to the growth of the outer shear layer. The shear layer, which grows approximately linearly with 


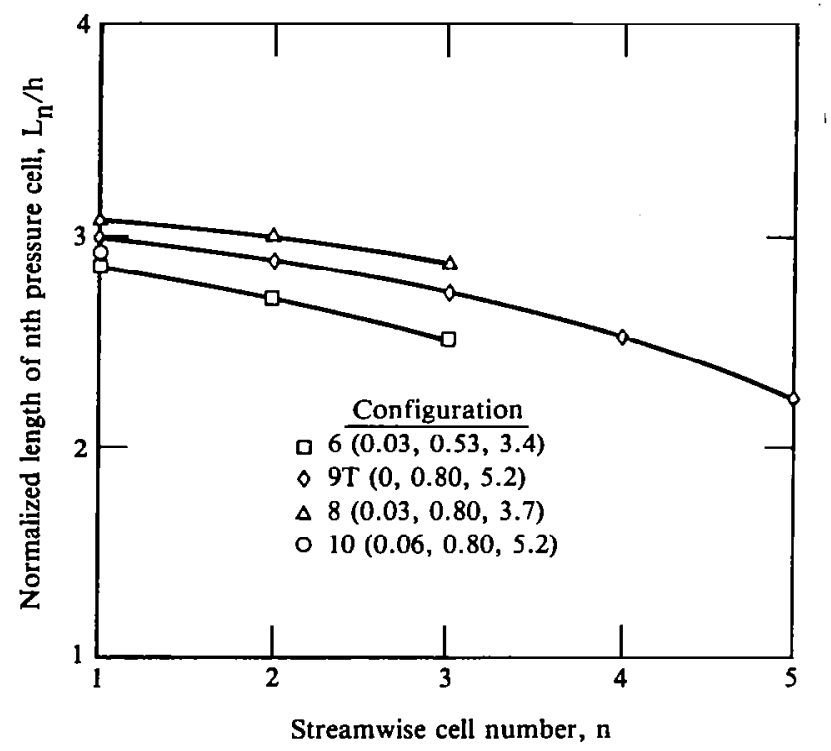

Figure 50. Streamwise variation of pressure cell length at a nozzle pressure ratio of 2.8 .

streamise distance, effectively reduces the cross-sectional area of the high-speed core flow.

The observation that the pressure cell spacing scales with the height of the annular exit and decreases with the local cross-sectional area of the supersonic core suggests a reflection mechanism for the compression-expansion wave system of the plug nozzle jet. The expansion fan emanating from the nozzle lip reflects from the plug surface as a more diffuse expansion. The expansion is reflected from the shear layer as a diffuse compression. In the case of the solid plug, the compression reflects from the plug surface as a discrete shock. When the plug surface is porous, the reflection from the plug is composed of weak shocks originating at the surface holes. The pattern repeats along the lengh of the plug, with the distance between reflections decreasing as the shear layer thickens (see Figure 51).

The reflection mechanism is significant in two respects: it explains the increasing unsteadiness of shock cell position with distance from the nozzle exit and the role of surface porosity in the suppression of noise. High speed shadowgraph movies show that the initial shock cell is the steadiest, with the range of unsteady cell motion nearly 


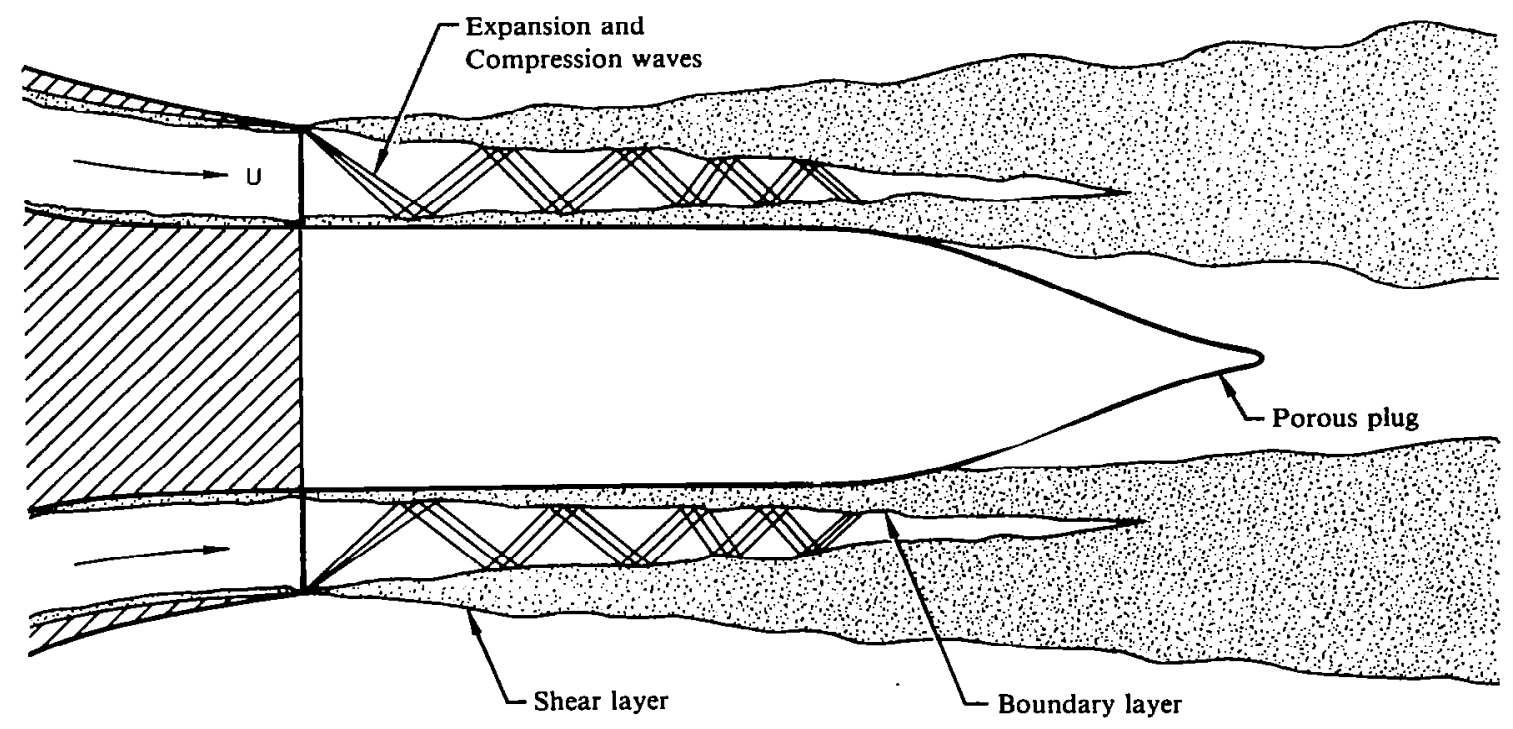

Figure 51. Porous plug nozzle flowfield.

doubling for each successive cell. If the cell position is the consequence of multiple reflections from the unsteady shear layer, each reflection increases the unsteadiness of the resultant wave. Although intense shock cell osclliation may be related to the screech phenomenon, it is not clear that the oscillation observed in the present study is directly linked to noise generation. The results of Seiner and Norum, 6 Pao and Seiner, 11 and Seiner and $\mathrm{Yu}^{12}$ indicate that the predominant source of broadband noise is the interaction between the shock cells and the vortical structure in the thick shear layer.

When viewed superficially, the role of surface porosity is not clear because the predominant sound sources are located in the jet shear layer; however, when viewed in terms of the reflection mechanism, the role of surface porosity becomes more distinct. Ribner ${ }^{13}$ proposed that a porous plug acts as a boundary that is intermediate between a hard and a soft surface. According to his hypothesis, the solid areas of the plug reflect an expansion as an expansion, whereas the porous regions act as soft surfaces and reflect an expansion as a shock. For a porous surface, the alternating regions of holes and solid area reflect shock and expansion waves that mutually cancel, and with appropriate surface 
porosity the net reflection of an impinging expansion wave is negligible.

In Figure 52 the normalized pressure ratio across compressions and expansions is presented as a function of normalized streamwise distance. The ratio is equivalent to shock strength, where $P_{1}$ is the pressure on the low-pressure side of the compression or expansion, and $P_{2}$ is the corresponding pressure on the high-pressure side. The compression strength gradually decays along the length of the solid plug, but the decay rate is small in comparison with that for the porous centerbodies. If the reflection of the compression and expansion waves were idea1 (that is, if the reflected wave were as strong as the incident wave), the pressure cell strength would remain constant. Interactions with the viscous shear layer and the plug surface boundary layer diminish the strength of the reflected waves, and multiple reflections lead to the eventual decay of the pressure cells.

When a solid plug is present in the jet, the axial wavelength of the cell structure is reduced. More reflections occur over a given axial distance than in the free jet; hence the cell strength at down-

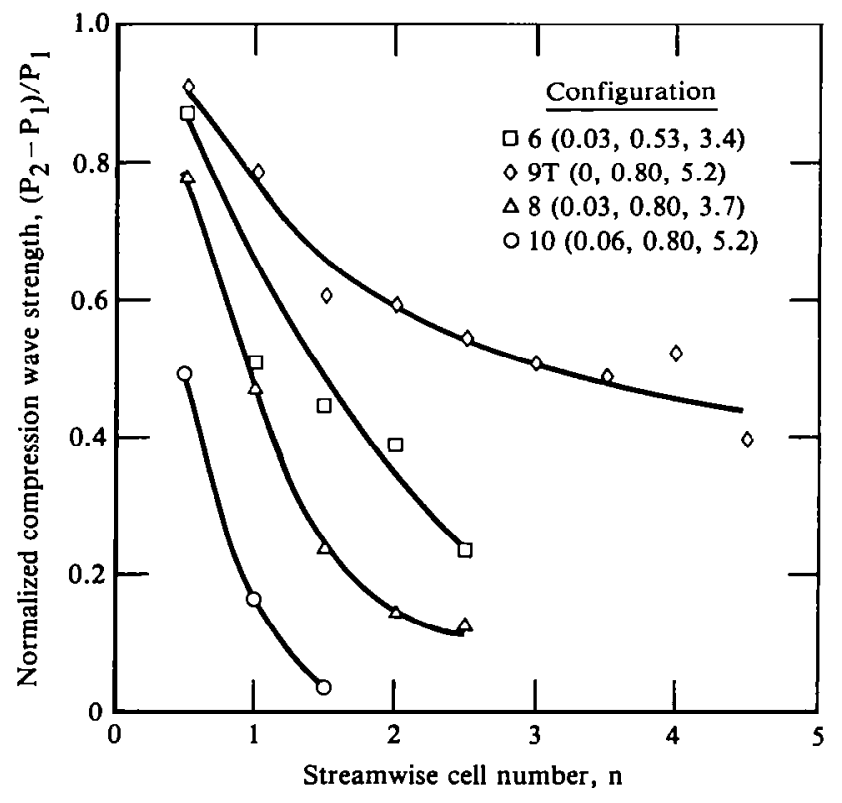

Figure 52. Streamwise variation of pressure cell strength at a nozzle pressure ratio of 2.8 . 
stream locations is reduced. The decay of the cell strength is even greater for a porous plug, and the decrease in reflected strength with increasing surface porosity is consistent with Ribner's hypothesis. When the surface porosity is $6 \%$, there are negligible reflections beyond the first cell. This characteristic is important for noise suppression in light of the mechanism of broadband noise generation outlined in References 6, 11 , and 12 . The most efficient noise production is a consequence of shock-turbulence interactions when the shear layer is thick. If the compression strength is dissipated near the exit of the jet where the shear layer is thin, the nolse generating interactions that occur further downstream will be suppressed.

The weak shocks associated with the surface holes are most distinct in regions of low static pressure. The local Mach number is greatest in low pressure regions, so shocks can be expected to be strongest there. Secondly, the pressure inside the plug is approximately atmospheric, and the variation in static pressure along the plug surface produces significant static pressure differentials and, consequently, flow through the holes. In regions of low static pressure, the flow exits from the plug in a series of small jets that induce the formation of weak shocks. A consequence of the mass flux through the plug surface may be additional thrust loss, although it is difficult to estimate the magnitude of such losses from the current experimental data. Thrust measurements show that losses increase with increasing plug porosity, but few details of the mechanism have been resolved.

It is ultimately desirable to relate the observed flow phenomena to noise production by the jet flow. The near-and far-field results show the dependence of sound production on the geometrical characteristics of the plug nozzles, and the aerodynamic results indicate that the degree of sound suppression is linked to the mean static pressure field. In Figure 53 the near-field sound pressure level in the jet exit plane is presented as a function of the nondimensional pressure increase through the first compression wave (the compression strength). In Figure 54, the far-field data obtained in the acoustic tests are presented in a similar plot. The far-field results collapse to a single line, 


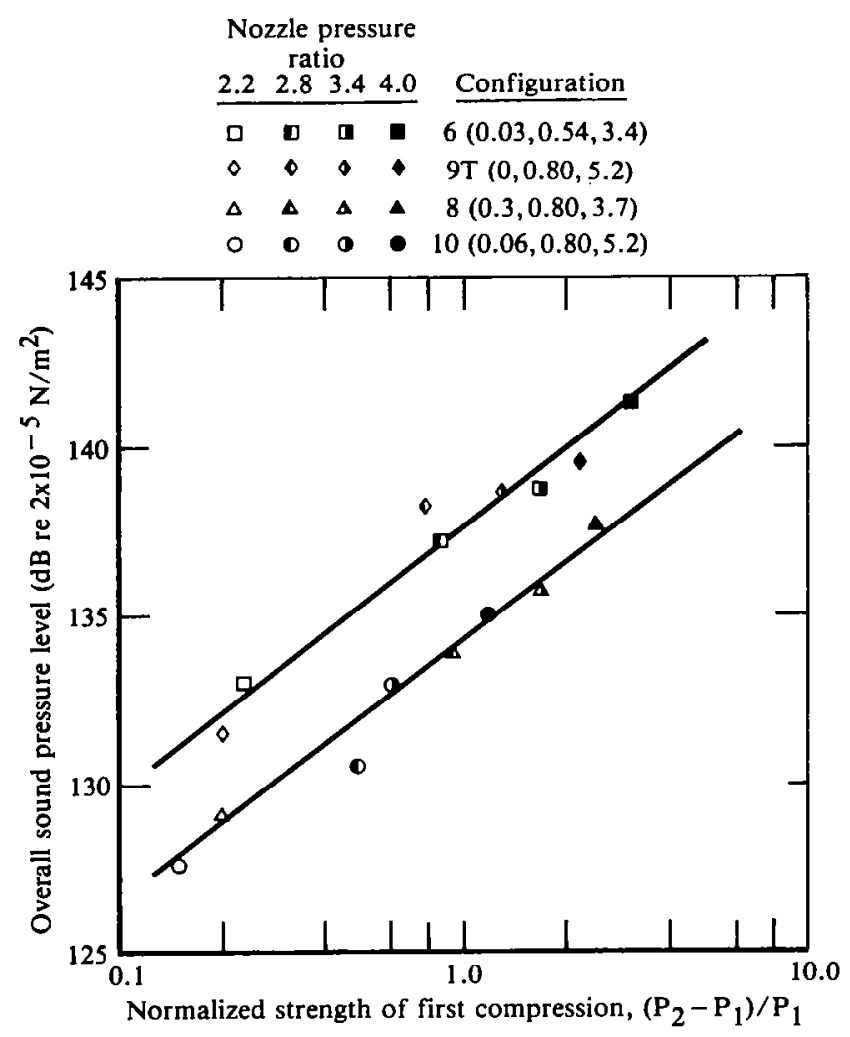

Figure 53. Variation of near-field sound pressure level with strength of first compression.

indicating a simple direct relationship between the noise level and the strength of the first pressure cell for all plug geometries. The overal1 sound level for the Configuration 10 nozzle at a pressure ratio of 4.0 is similar to the sound level for the Configuration 9T nozzle at a pressure ratio of 2.8 because the pressure increase through the first compression is nearly the same for both cases. The fact that the overall noise level scales with the pressure cell strength indicates that the cells are linked to the primary source of supersonic noise in the plug nozzle flow, although the detailed mechanism can be expected to be similar to the interaction between the shear layer and shock waves documented by Seiner and Norum. 6

If the noise suppression characteristics of a porous plug nozzle are to be maximized, the strength of the pressure cells must be reduced. Although the overall effect is linked to the cell strength, other fac- 


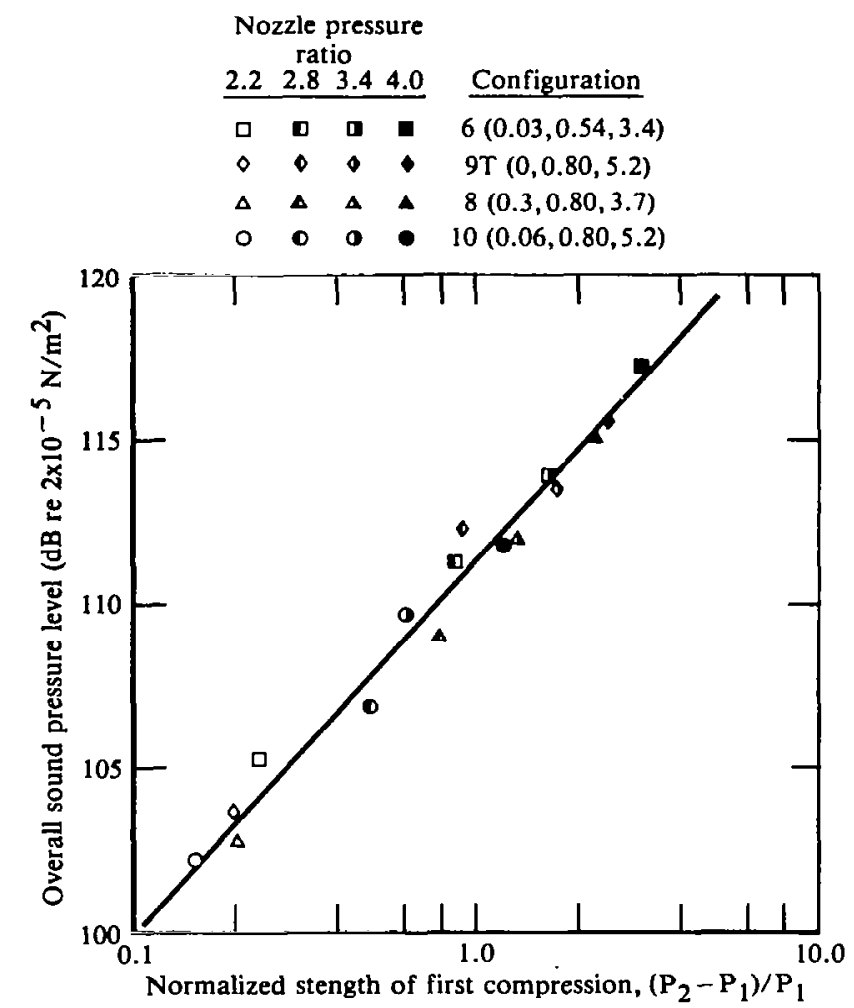

Figure 54. Variation of far-field sound pressure level with strength of first compression.

tors such as cell persistence and unsteadiness can contribute to the noise reduction. It is likely that pressure cells beyond the first are dominant noise sources, as evidenced by the near-field measurements in Figure 53. Both configurations which fall along the curve of higher noise production are known to have multiple strong compression cells. On the basis of the present results, it appears probable that a prudent choice of surface porosity, plug-to-nozzle diameter-ratio, and plug length will result in quieter nozzle configurations with less thrust loss than current designs. 


\section{CONCLUSIONS}

1. The measured sound pressure levels are belleved to be reasonably accurate, since repeats of test conditions generally provided onethird octave band sound pressure levels within $\pm 0.2 \mathrm{~dB}$ of the earlier results.

2. The effects of the flow conditions and plug geometry on the jet noise may be summarized as follows:

a) Porous plug suppressors with a large radius ratio (0.80) resulted in more sound reduction than suppressors with a smaller radius ratio (0.53), but thrust losses were greater for the larger ratio. At $90^{\circ}$ from the jet axis, the best large-radius-ratio plugs reduced the OASPL by $2.4,8.3,15.6,17.5$, and $12.6 \mathrm{~dB}$ at pressure ratios of $1.60,2.20,2.80,3.40$, and 4.00 , respective1y. The corresponding reductions for the best small-radius-ratio plugs were $1.2,6.1,12.2,14.1$, and $7.9 \mathrm{~dB}$. Nozzle thrust coefficients for the porous plugs were highest at pressure ratios of 2.20 and 2.80 .

b) A hard-walled plug reduced the noise significantly, but in a somewhat erratic fashion. For example, the reduction in OASPL at $90^{\circ}$ from the jet was $6.9 \mathrm{~dB}$ for a pressure ratio of 2.20 , but it was $1.0 \mathrm{~dB}$ for a pressure ratio of 2.80 and $8.4 \mathrm{~dB}$ for a pressure ratio of 3.40 .

c) A plug with a porosity or open area ratio of $5.6 \%$ reduced the noise more than a similar plug with a porosity of $2.7 \%$. The reduction in OASPL at $90^{\circ}$ to the jet was approximately $2 \mathrm{~dB}$ greater at pressure ratios of 2.80 to 4.00 ; the reduction was smaller at lower pressure ratios. However, the larger porosity also resulted in a reduction of jet thrust.

d) Thickening the wall of the porous plug both reduced the noise and increased the thrust.

e) Lengthening of the plugs resulted in some noise increases and in thrust losses; useful plugs could probably be shorter than those tested. 
f) Hot flow conditions generally reduced the suppression.

g) Forward-motion flow increased the suppression for a pressure ratio of 2.80 , but it decreased the suppression at other pressure ratios.

3. The following conclusions are made on the basis of the aerodynamic test results:

a) Velocity, flowfield static pressure data, and surface static pressure measurements demonstrate that the annular core flow of a plug nozzle jet is dominated by a periodic pressure cell structure. Although the structure is not immediately evident in shadowgraph visualization, it is analogous to the shock bottle structure occurring in a free supersonic jet.

b) Distinct shock waves occur only for the solid plug; the static pressure for the porous plugs has a streamwise variation that can be characterized as a damped sinusoid. The flow remains supersonic throughout the annular core and undergoes a serles of expansions and compressions along the length of the plug.

c) The length of the compression cells scales with the radial distance between the plug surface and the nozzle lip, and decreases with distance from the nozzle exit. The latter result is a consequence of shear layer growth and indicates that the compression cells result from the repeated reflection of compression and expansion waves from the shear layer and the plug surface.

d) Increasing either the surface porosity or the plug-to-nozzle diameter-ratio decreases the strength of the compression cells. In the case of a 0.80 diameter ratio nozzle with a solid-surface plug, the cells persist for at least six cycles, whereas no cells are observed beyond the initial compression for a similar plug with a $6 \%$ porosity surface. The porous surface attenuates compression-expansion wave reflections, in accordance with the mechanism proposed by Ribner. 11 
e) The near-and far-field overall sound pressure level data correlate with the strength of the initial compression for all plug geometries and nozzle pressure ratios. The far-field acoustic data collapse to a single power-law curve when plotted as a function of compression cell strength. Noise reduction by a supersonic plug nozzle is therefore related directly to the degree that the pressure cells are weakened.

f) The apparent discrepancy between the shadowgraphs and other flow measurements can be attributed to the differing sensitivities of the techniques. As Maestrello ${ }^{2}$ observed, shadowgraphs show that the porous plug flowfield is generally free of shock waves, but the visualization is not sensitive to the slow variations of pressure and velocity observable through other techniques. A digital filter was applied to the static pressure data to separate the smal1- and large-scale components of the pressure field; the large scales were found to be dominant.

g) The small-scale pressure field structure, which is linked to the hole pattern in the porous plugs, explains the absence of we11defined shocks. In regions where the large-scale pressure is much below ambient, the pressure difference between the plug interior and the annular core flow induces jet flow out through the surface of the plug. The jets lead to a series of two to three weak shock waves, producing a gradual static pressure rise.

h) The pressure cell position becomes increasingly unsteady with distance from the nozzle exit, particularly for the solid plug. The unsteadiness can be attributed to multiple compressionexpansion wave reflections from an unsteady shear layer. The holes in the porous plug act to stabilize the pressure cell position, because the weak shocks are tied to discrete hole locations. 


\section{REFEREWCES}

1. L. Maestrello. Initial Results of a Porous Plug Nozzle for Supersonic Jet Noise Suppression. NASA TM-78802 (1978).

2. L. Maestre11o. An Experimental Study on Porous Plug Jet Noise Suppressors. AIAA Paper No. 79-0673 (1979).

3. A. B. Bauer, M. R. Ballard, J. H. Brettnacher, and V. Kibens. The Development and Operation of the MDC Anechoic Acoustic Test Facility. AIAA Paper No. 80-0982 (1980).

4. P. A. Lush. Measurements of Subsonic Jet Noise and Comparison with Theory. J, of Fluid Mech., 46, Part 3, pp. 477-500 (1971).

5. S. Z. Pinckney. A Short Static-Pressure Probe Design for Supersonic Flow. NASA TN D-7978 (1975).

6. J. M. Seiner and T. D. Norum. Aerodynamic Aspects of Shock Containing Jet Plumes. AIAA Paper No. 80-0965 (1980).

7. C. Y. Tsui and G. A. Flandro. Self-Induced Sound Generation by Flow Over Perforated Duct Liners. J. of Sound and Vibration, 50, pp. 315-331 (1950).

8. A. B. Bauer and R. L. Chapkis. Noise Generated by Boundary Layer Interaction with Perforated Acoustic Liners. AIAA J. of Aircraft, 14, pp. 157-160 (1977).

9. M. J. Lighthil1. On the Energy Scattered from the Interaction of Turbulence with Sound or Shock Waves. Proc. Camb. Phil. Soc., 49, pp. 531-551 (1953).

10. M. S. Howe and J. E. Ffowcs-Williams. On the Noise Generated by an Imperfectly Expanded Supersonic Jet. Phil. Trans. R. Soc. Lond. A 289, pp. 271-314 (1978).

11. P. S. Pao and J. M. Seiner. A Theoretical and Experimental Investigation of Shock-Associated Noise in Supersonic Jets. AIAA Paper No. 81-1973 (1981).

12. J. M. Selner and J. C. Yu. Acoustic Near Field and Local Flow Properties Associated with Broadband Shock Noise. AIAA Paper No. 81-1975 (1981).

13. H. S. Ribner. Perspectives on Jet Noise. AIAA J., 19, 12, pp. 1513-1526 (1981). 
14. H. Schlichting. Boundary-Layer Theory. McGraw-Hill (1968).

15. U. Michel and A. Michalke. Prediction of Flyover Jet Noise Spectra from Static Tests. NASA TM-83219 (1981). 
APPENDIX A

SOUND PRESSURE LEVELS AS A FUNCTION OF ONE-THIRD OCTAVE BAND FREQUENCY

Figure Numbers

A1 through A36

A37 through A44

A45 through A52

A53 through A64

A65 through A72
Data Description

Cold Flow, No Forward Motion

Hot Flow $\left(370^{\circ} \mathrm{C}\right)$, No Forward Motion

Hot Flow $\left(540^{\circ} \mathrm{C}\right)$, No Forward Motion

Cold Flow with Forward Motion Effects

Cold Flow with Acoustic Shield 


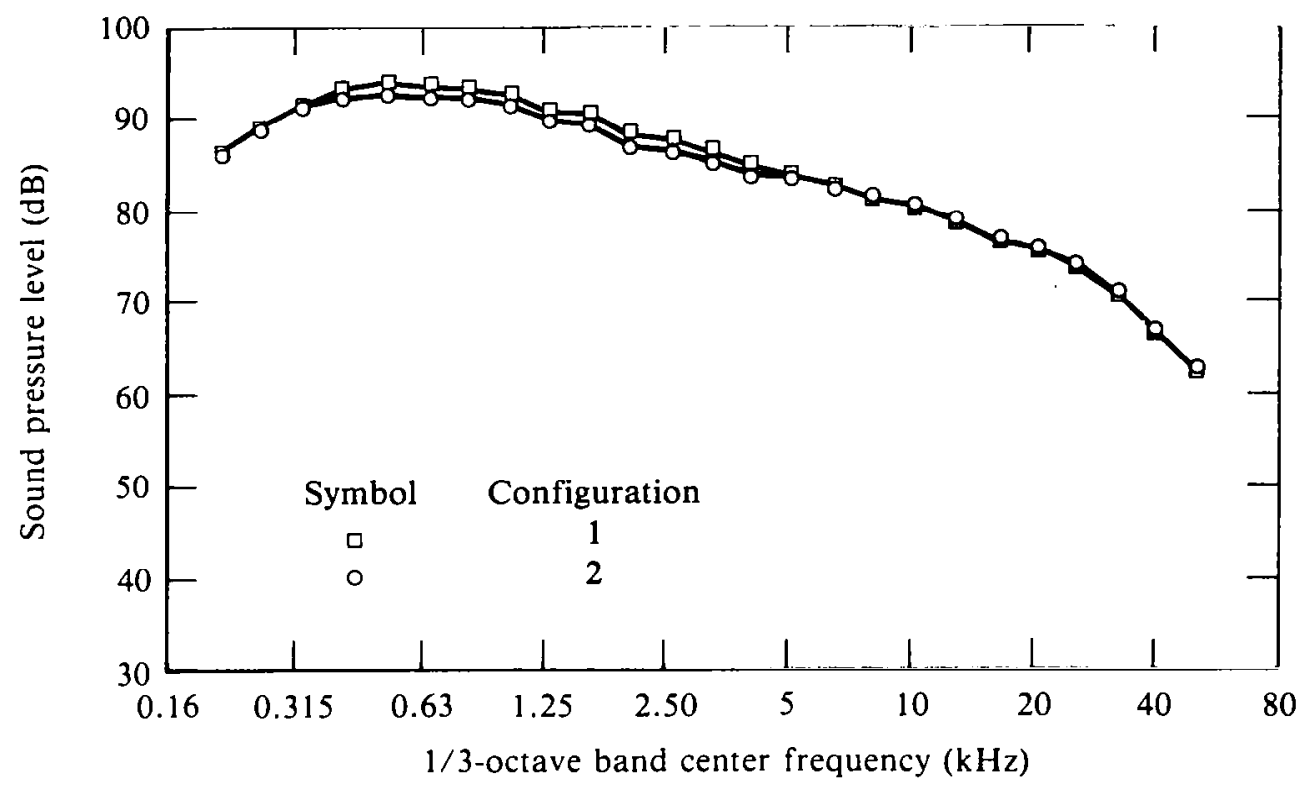

Figure A1. Sound pressure levels as a function of 1/3-octave band frequency; microphone 3 , pressure ratio $=1.6$, cold flow, and no forward motion.

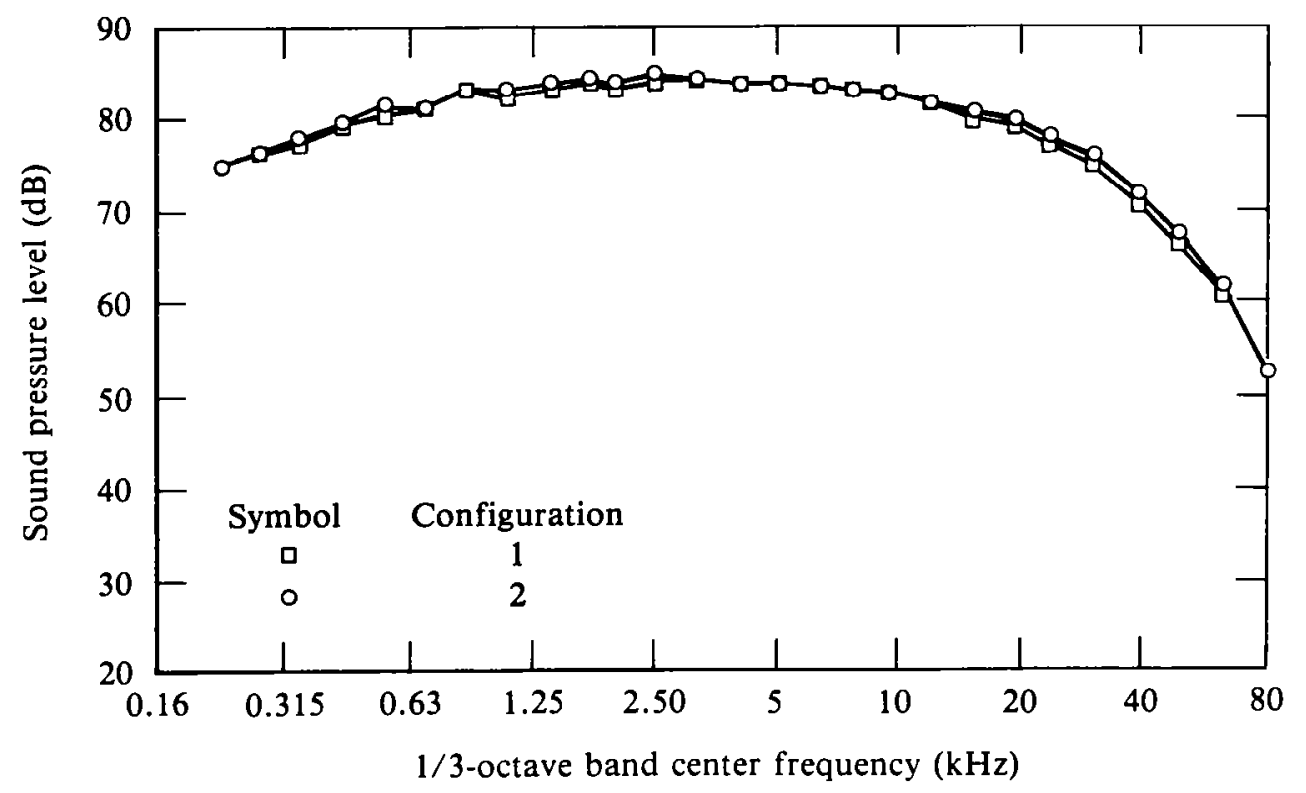

Figure A2. Sound pressure levels as a function of 1/3-octave band frequency; microphone 9 , pressure ratio $=1.6$, cold flow, and no forward motion. 


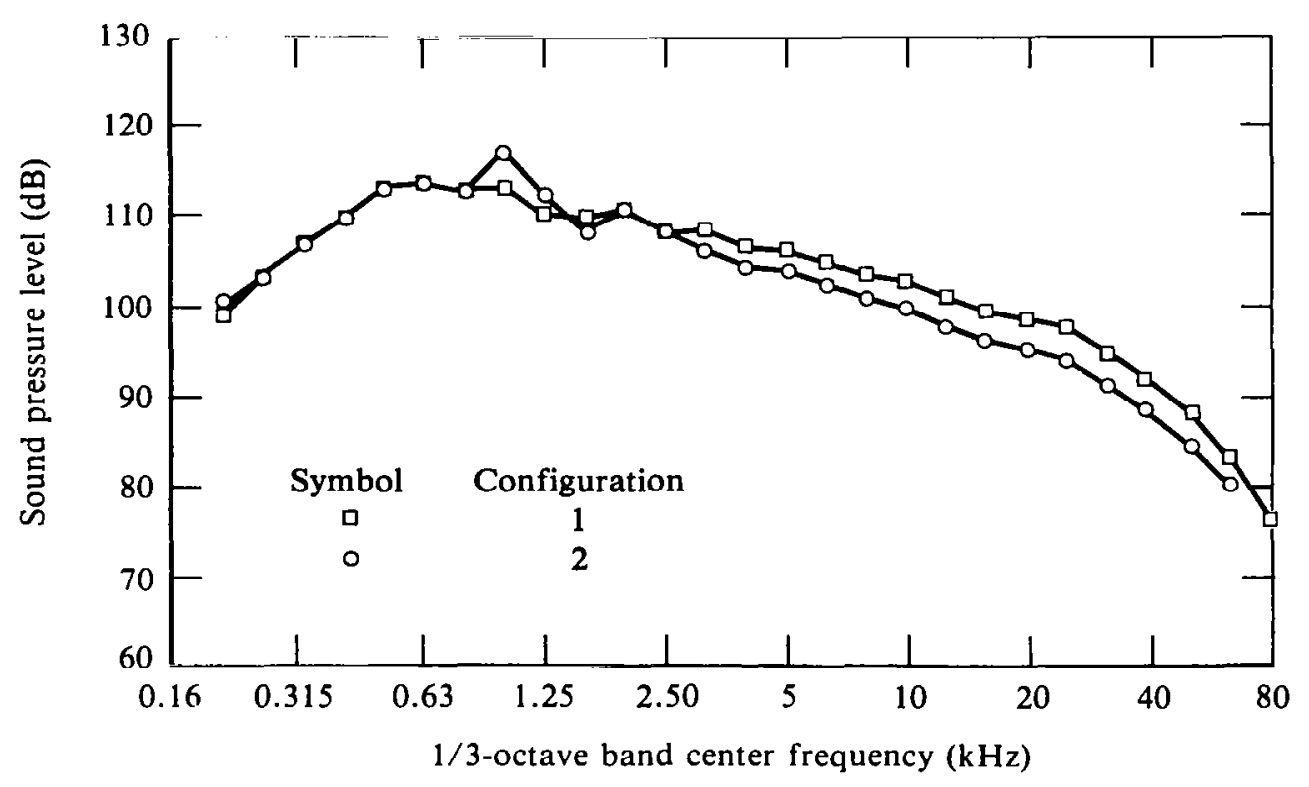

Figure A3. Sound pressure levels as a function of 1/3-octave band frequency; microphone 3 , pressure ratio $=2.8$, cold flow, and no forward motion.

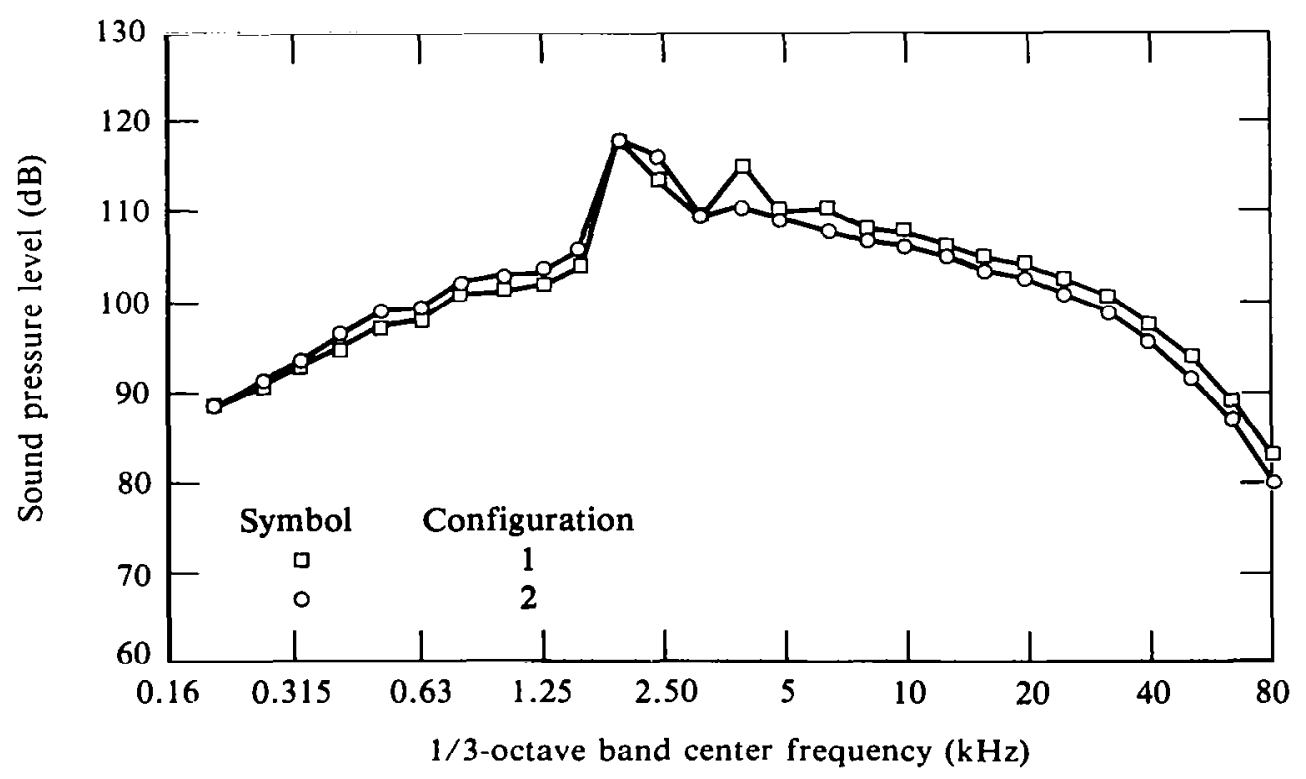

Figure A4. Sound pressure levels as a function of 1/3-octave band frequency; microphone 9 , pressure ratio $=2.8$, cold flow, and no forward motion. 


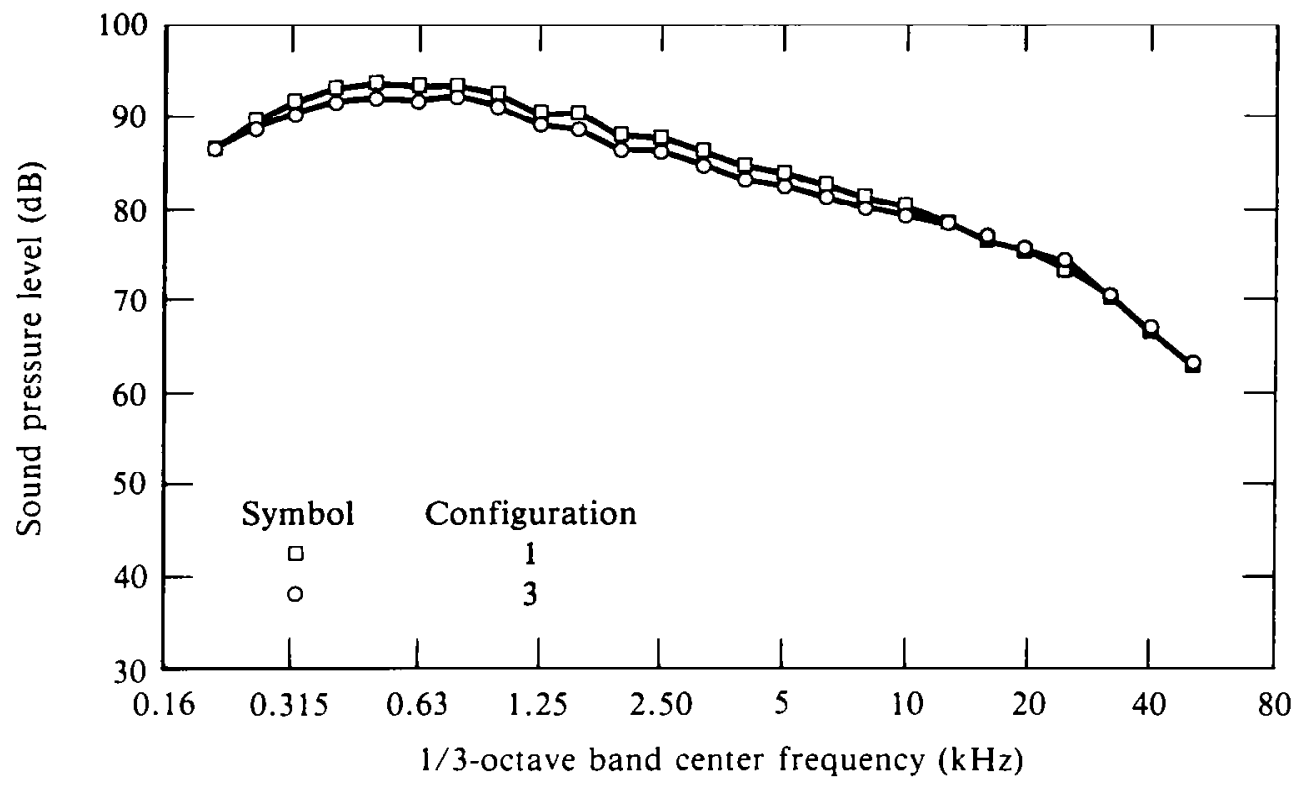

Figure A5. Sound pressure levels as a function of 1/3-octave band frequency; microphone 3 , pressure ratio $=1.6$, cold flow, and no forward motion.

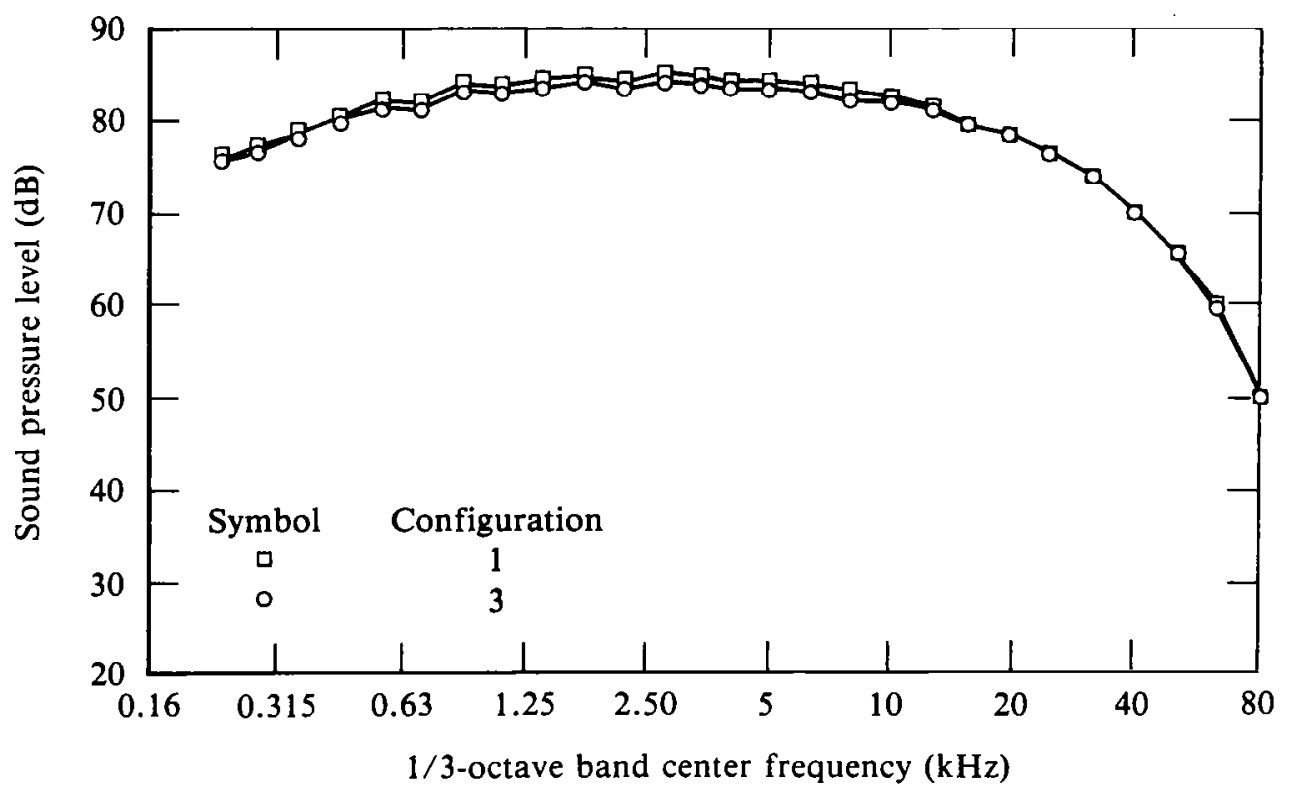

Figure A6. Sound pressure levels as a function of 1/3-octave band frequency; microphone 9 , pressure ratio $=1.6$, cold flow, and no forward motion. 


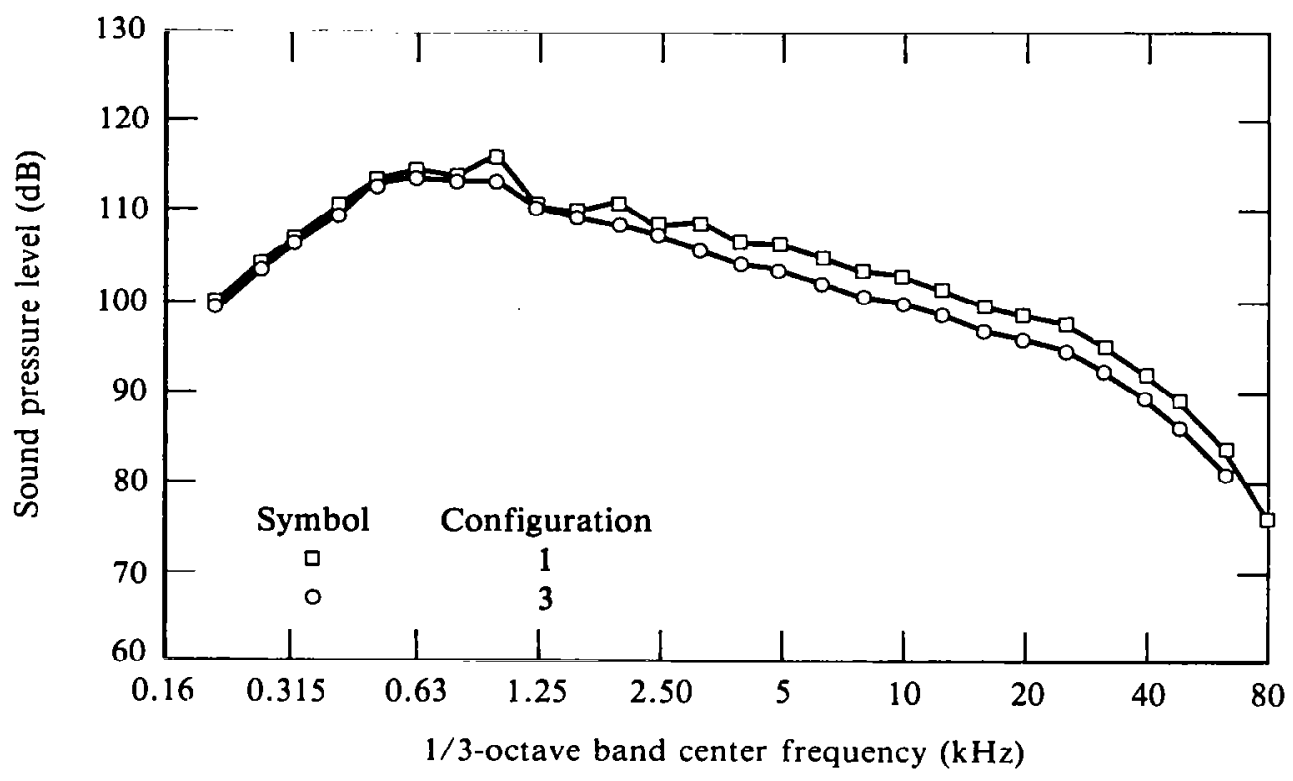

Figure A7. Sound pressure levels as a function of 1/3-octave band frequency; microphone 3 , pressure ratio $=2.8$, cold flow, and no forward motion.

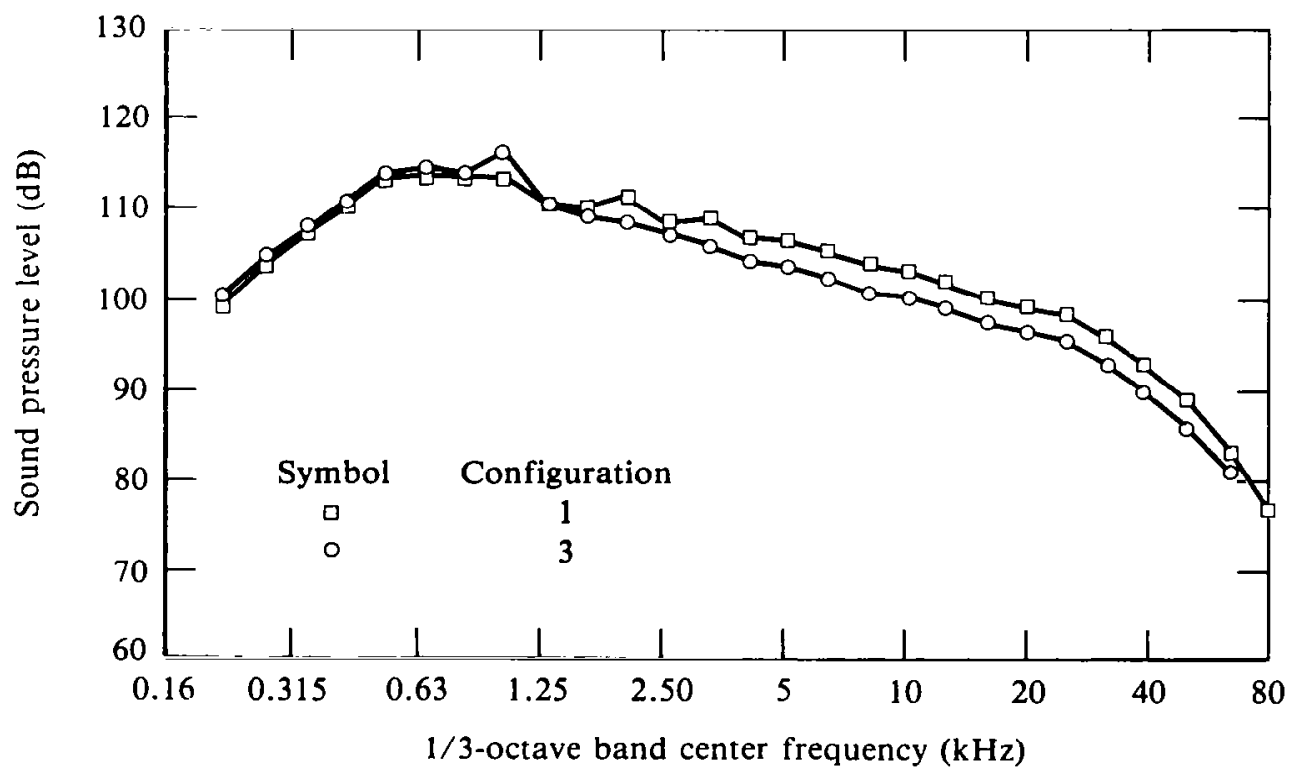

Figure A8. Sound pressure levels as a function of 1/3-octave band frequency; microphone 9 , pressure ratio $=2.8$, cold flow, and no forward motion. 


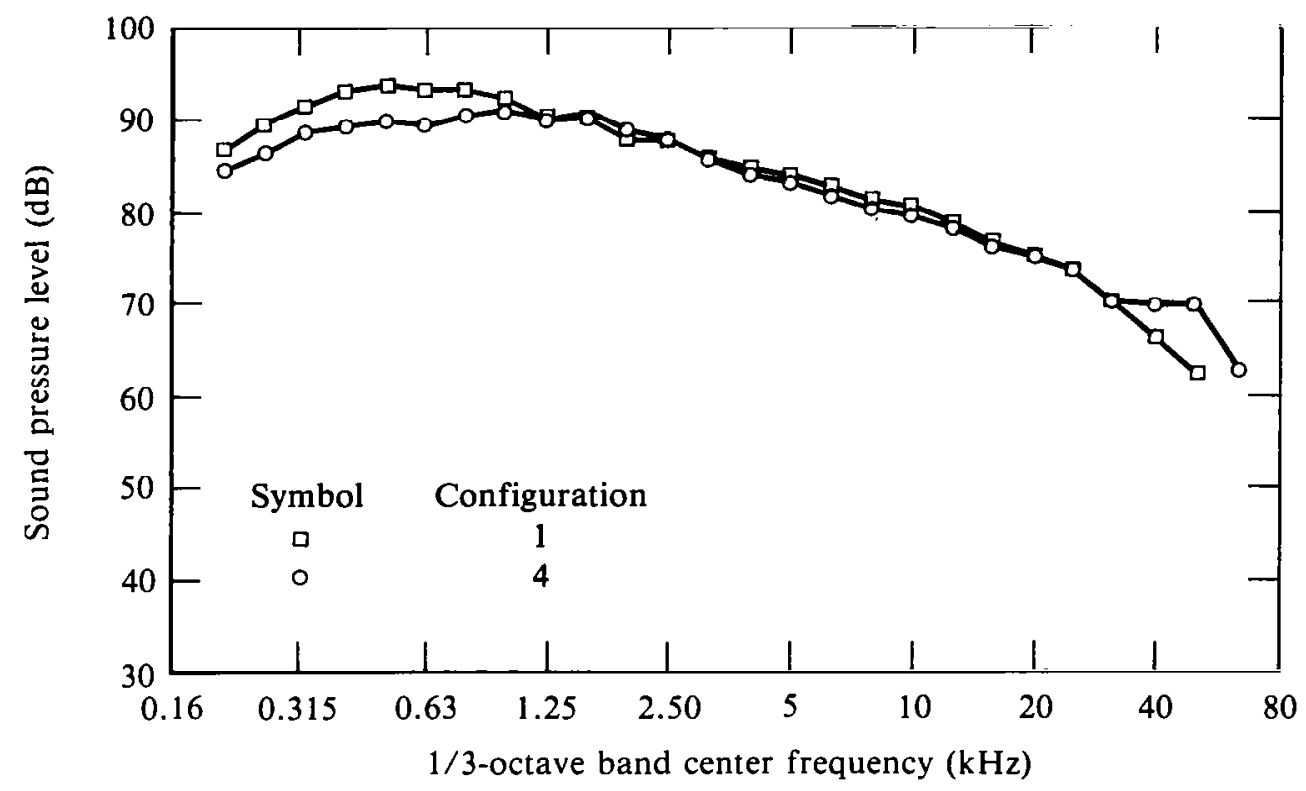

Figure A9. Sound pressure levels as a function of 1/3-octave band frequency; microphone 3 , pressure ratio $=1.6$, cold flow, and no forward motion.

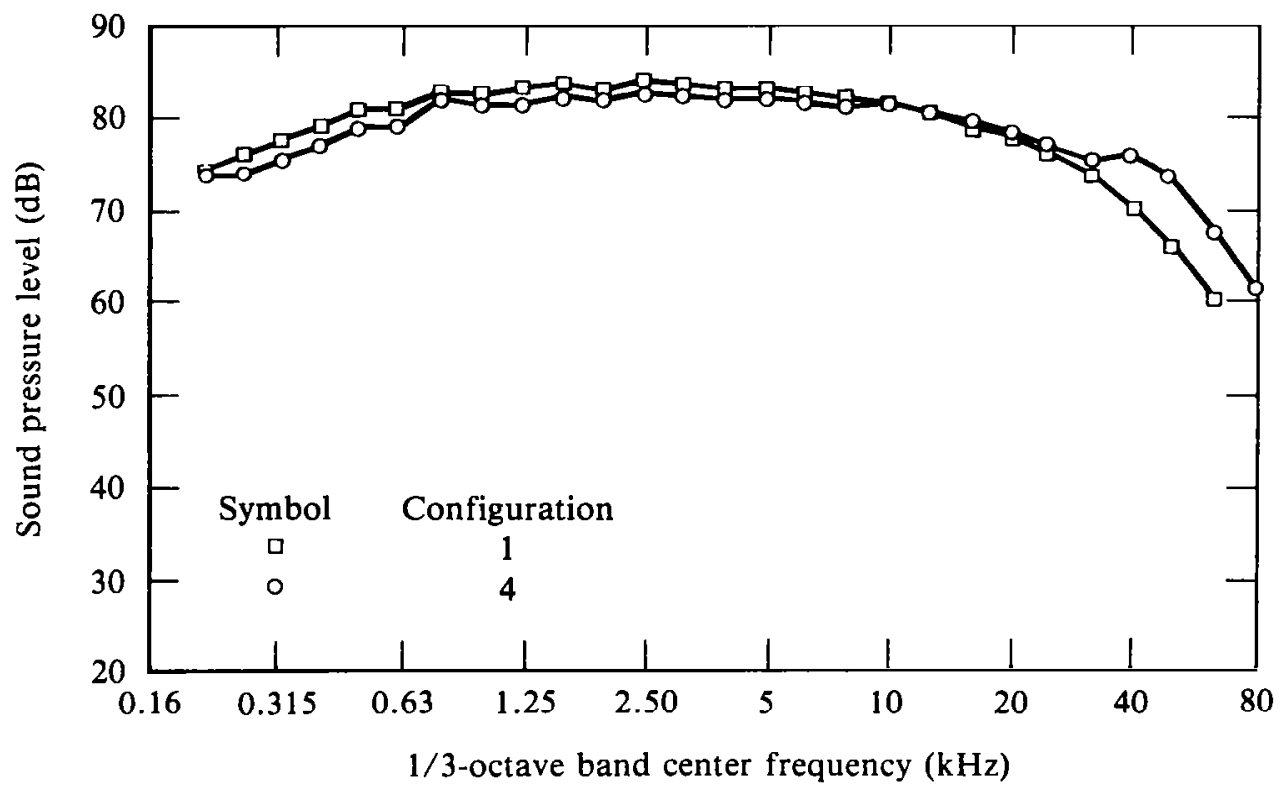

Figure A10. Sound pressure levels as a function of 1/3-octave band frequency; microphone 9 , pressure ratio $=1.6$, cold flow, and no forward motion. 


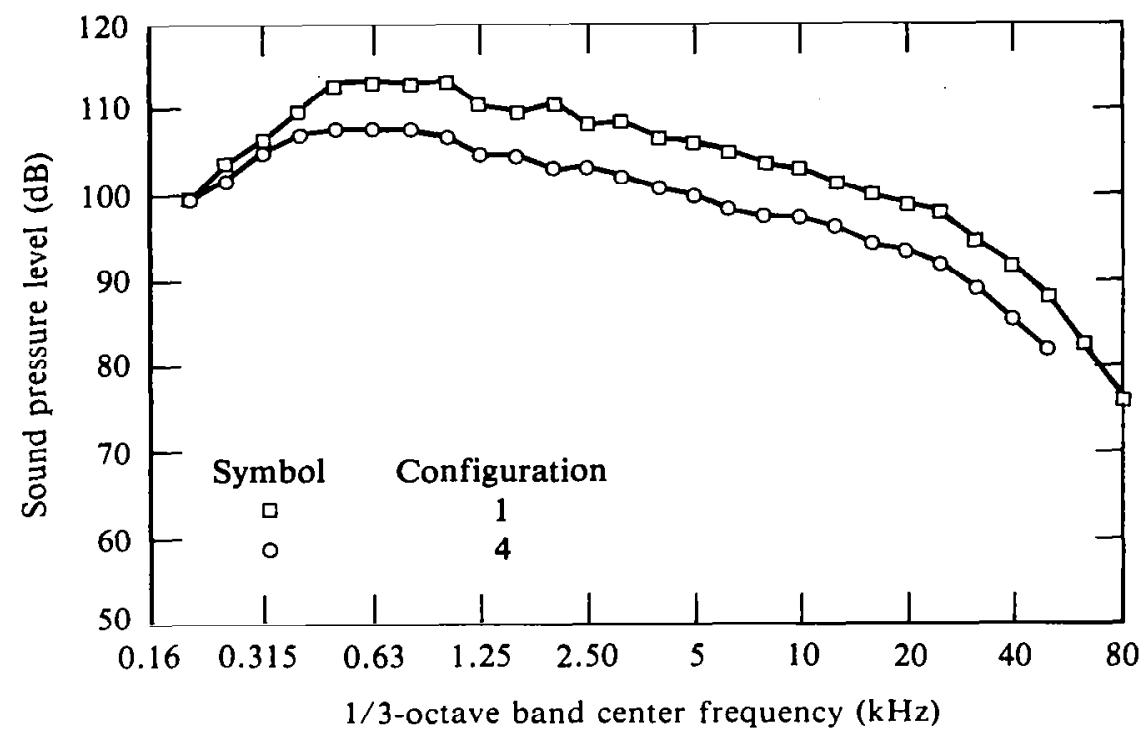

Figure A11. Sound pressure levels as a function of 1/3-octave band frequency; microphone 3 , pressure ratio $=2.8$, cold flow, and no forward motion.

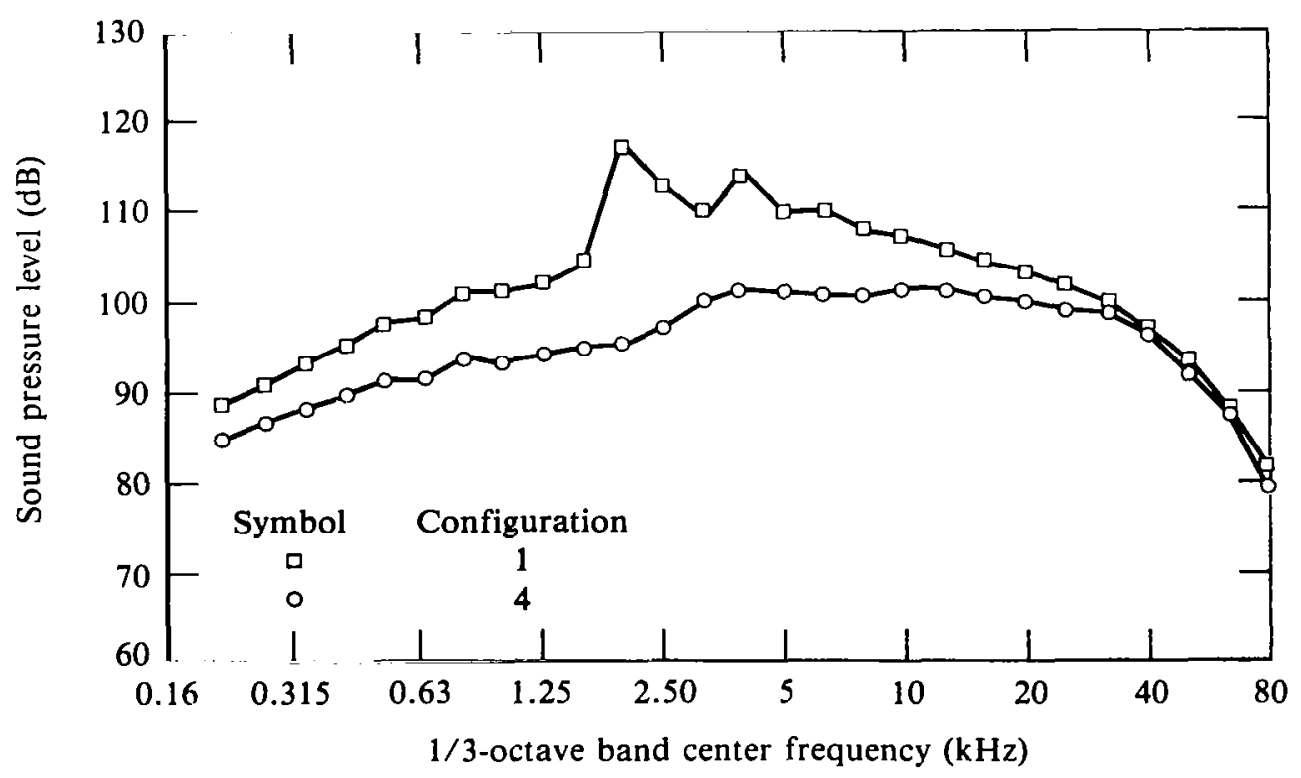

Figure A12. Sound pressure levels as a function of 1/3-octave band frequency; microphone 9 , pressure ratio $=2.8$, cold flow, and no forward motion. 


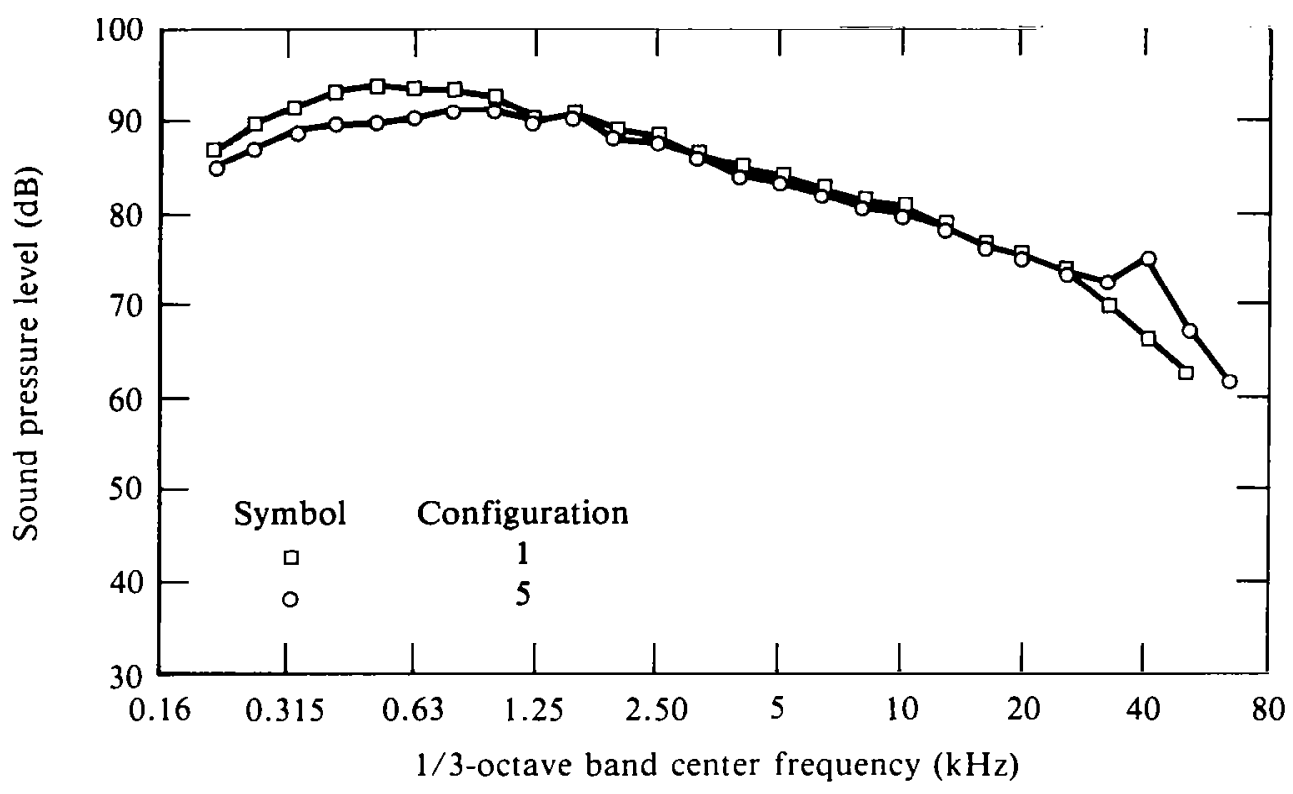

Figure A13. Sound pressure levels as a function of $1 / 3$-octave band frequency; microphone 3 , pressure ratio $=1.6$, cold flow, and no forward motion.

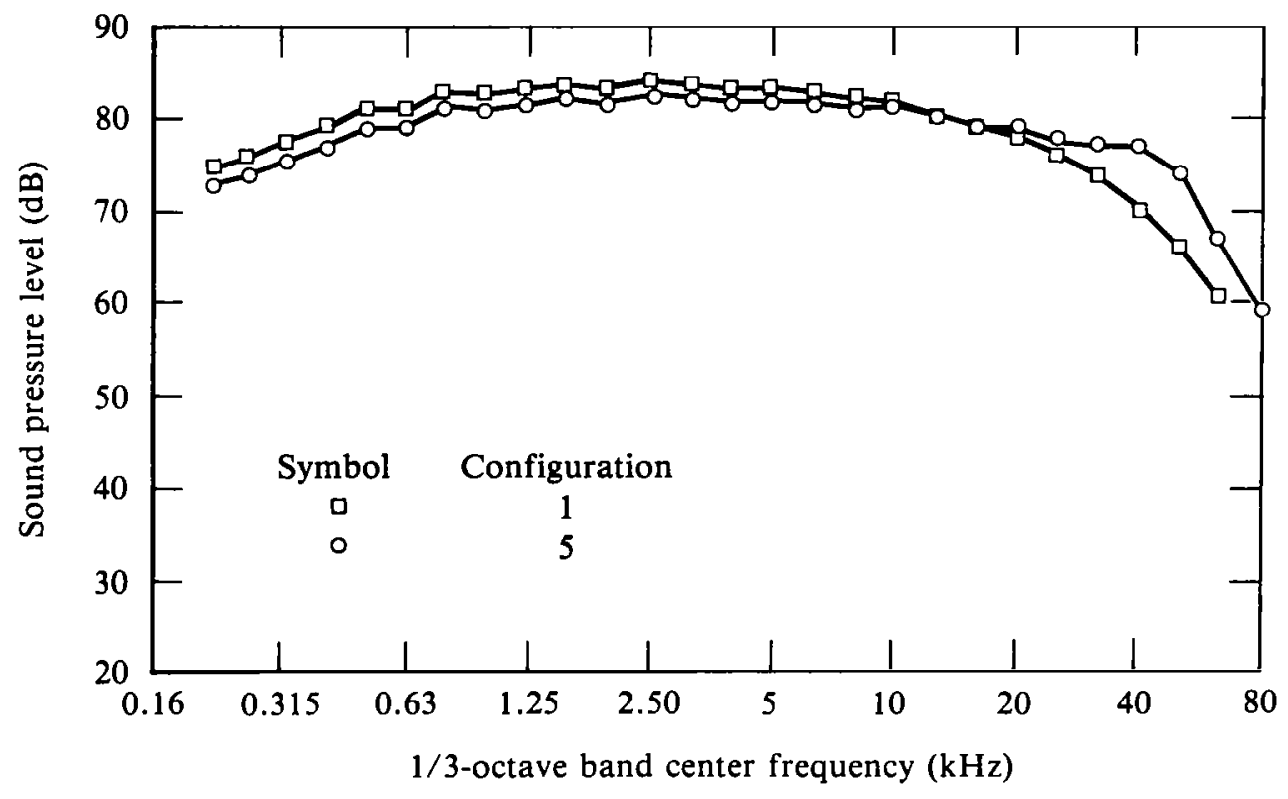

Figure A14. Sound pressure levels as a function of 1/3-octave band frequency; microphone 9 , pressure ratio $=1.6$, cold flow, and no forward motion. 


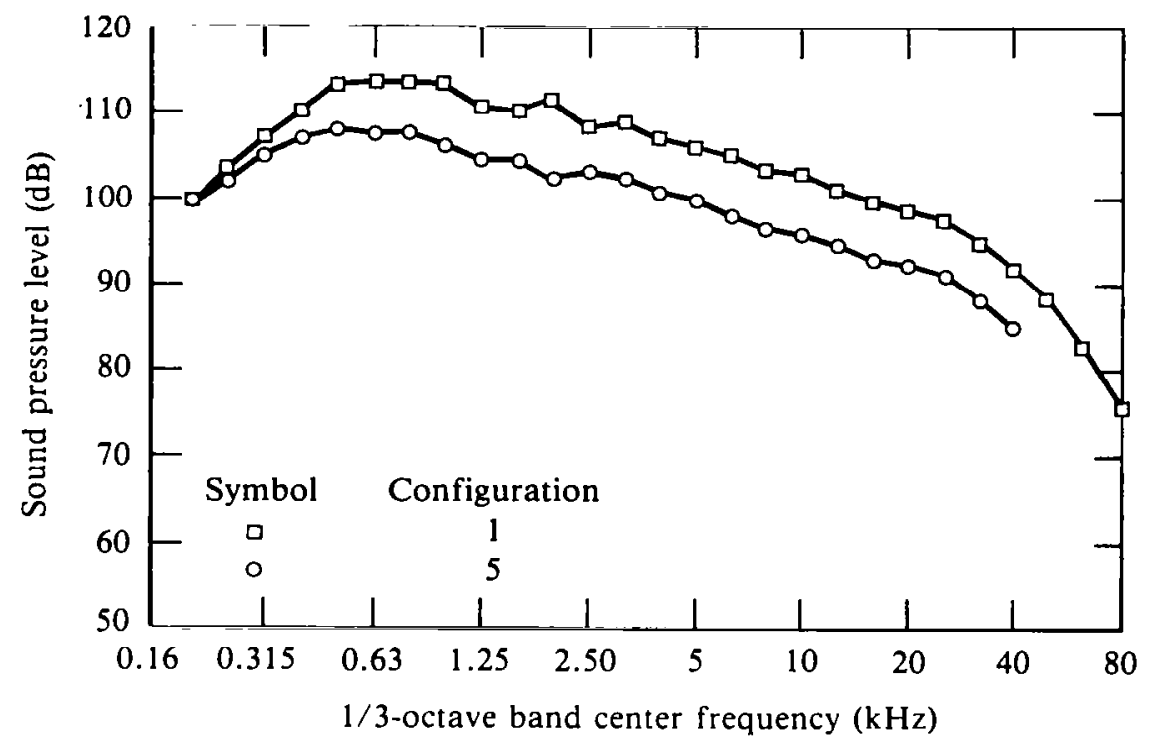

Figure A15. Sound pressure levels as a function of 1/3-octave band frequency; microphone 3 , pressure ratio $=2.8$, cold flow, and no forward motion.

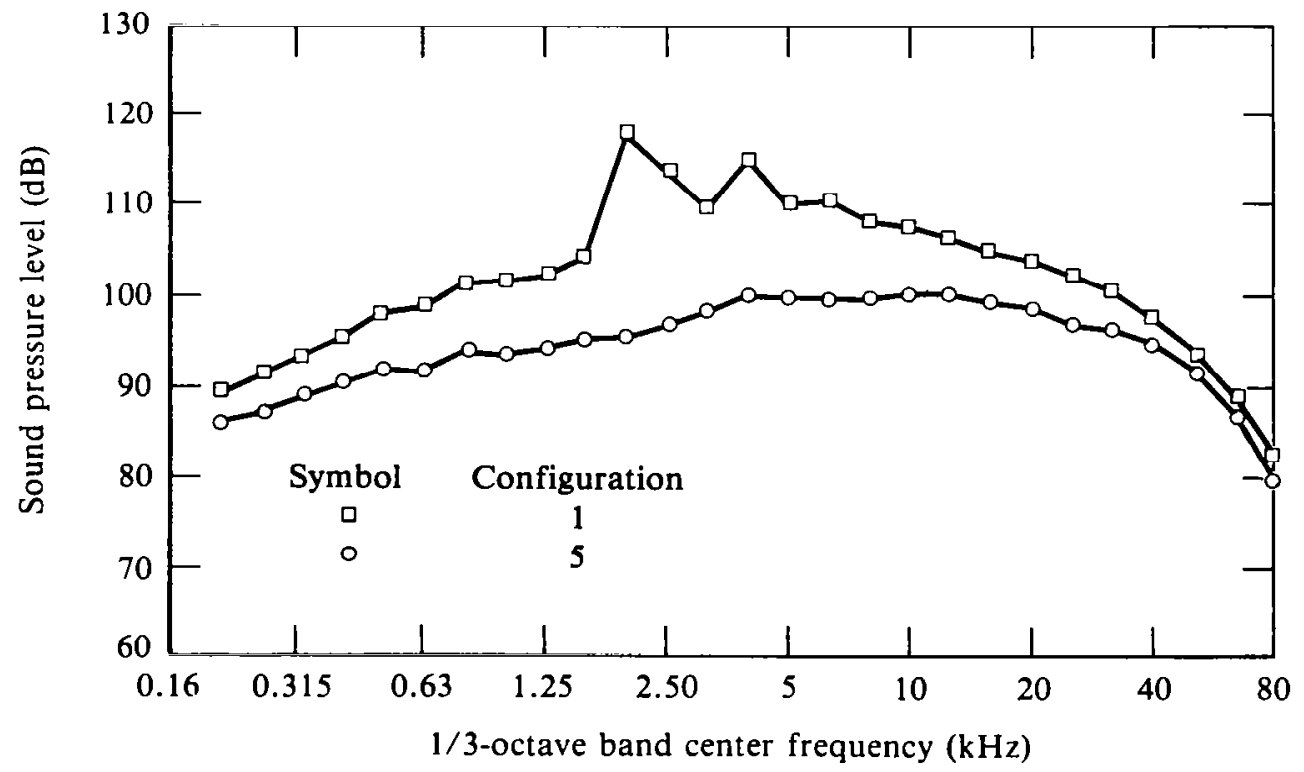

Figure A16. Sound pressure levels as a function of 1/3-octave band frequency; microphone 9 , pressure ratio $=2.8$, cold flow, and no forward motion. 


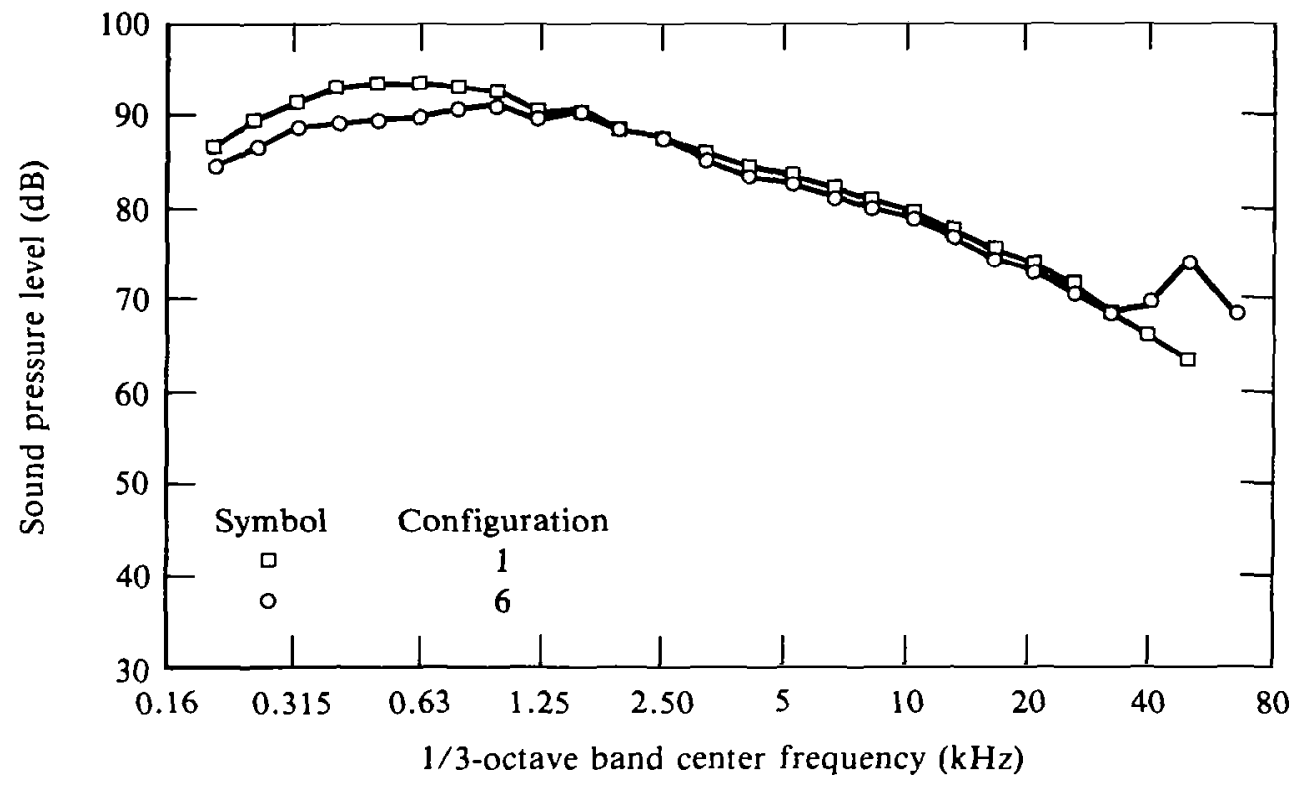

Figure A17. Sound pressure levels as a function of $1 / 3$-octave band frequency; microphone 3 , pressure ratio $=1.6$, cold flow, and no forward motion.

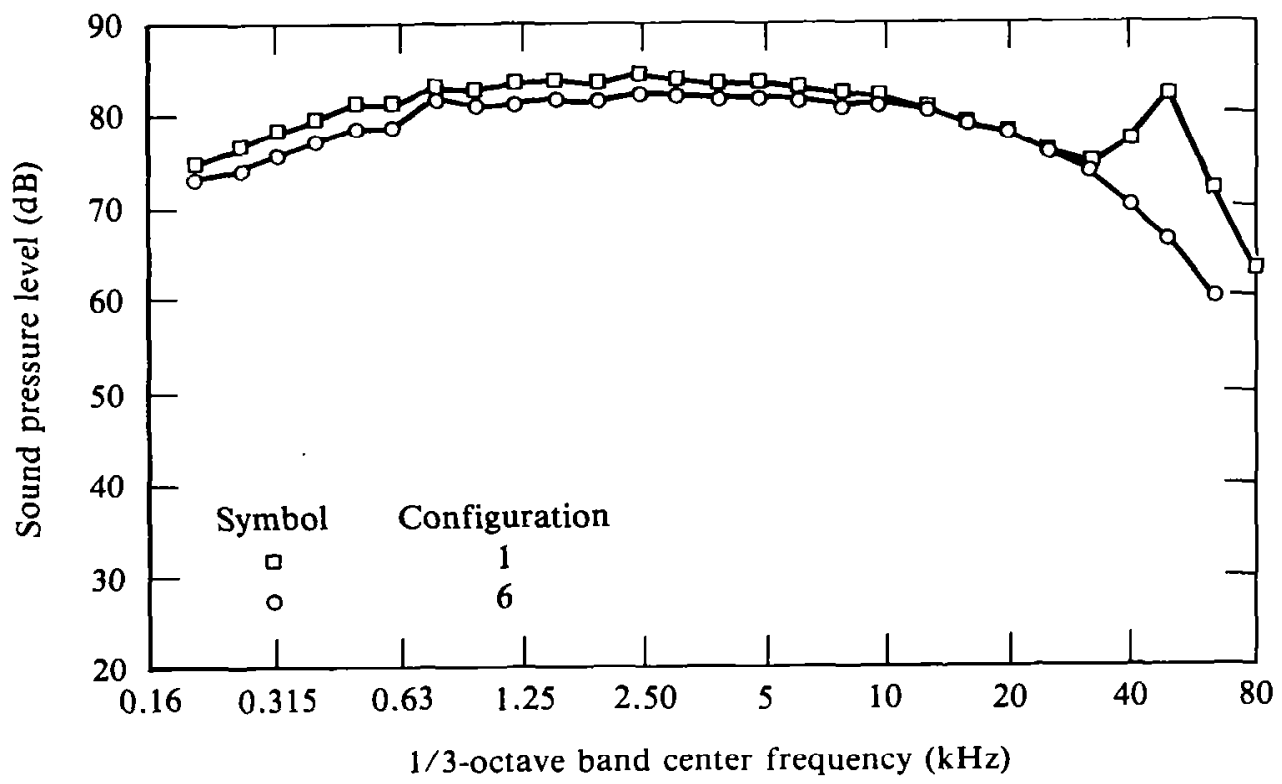

Figure A18. Sound pressure levels as a function of 1/3-octave band frequency; microphone 9 , pressure ratio $=1.6$, cold flow, and no forward motion. 


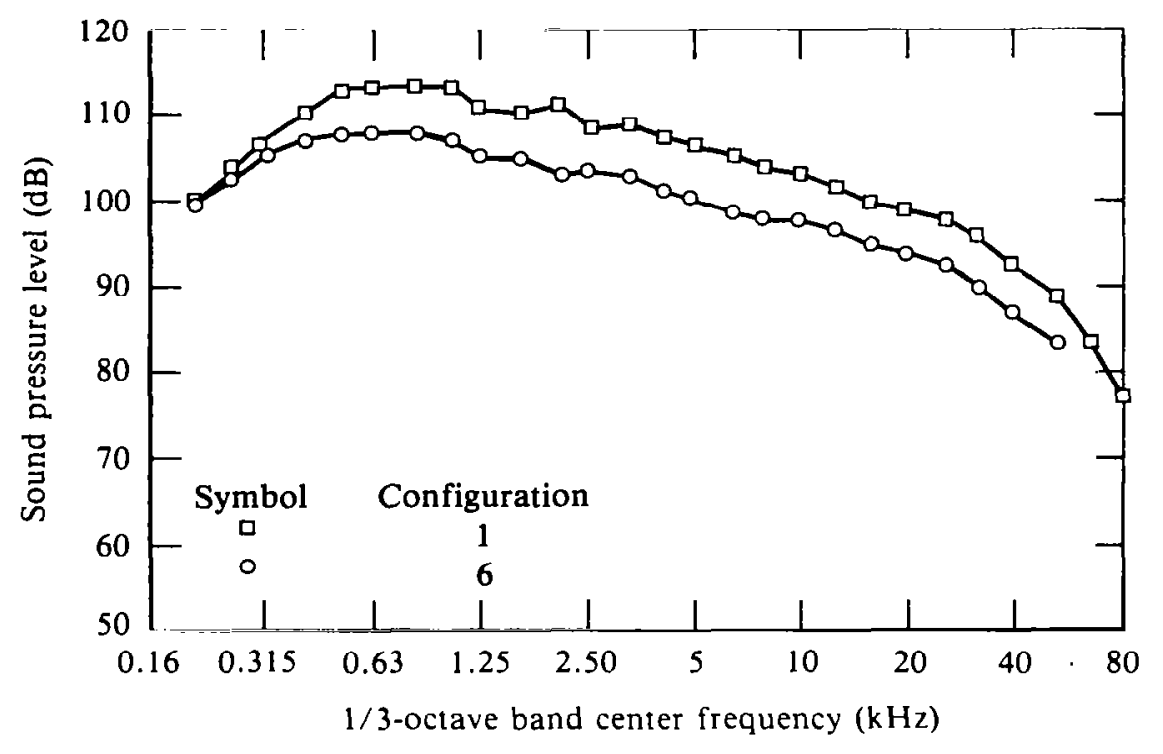

Figure A19. Sound pressure levels as a function of 1/3-octave band frequency; microphone 3 , pressure ratio $=2.8$, cold flow, and no forward motion.

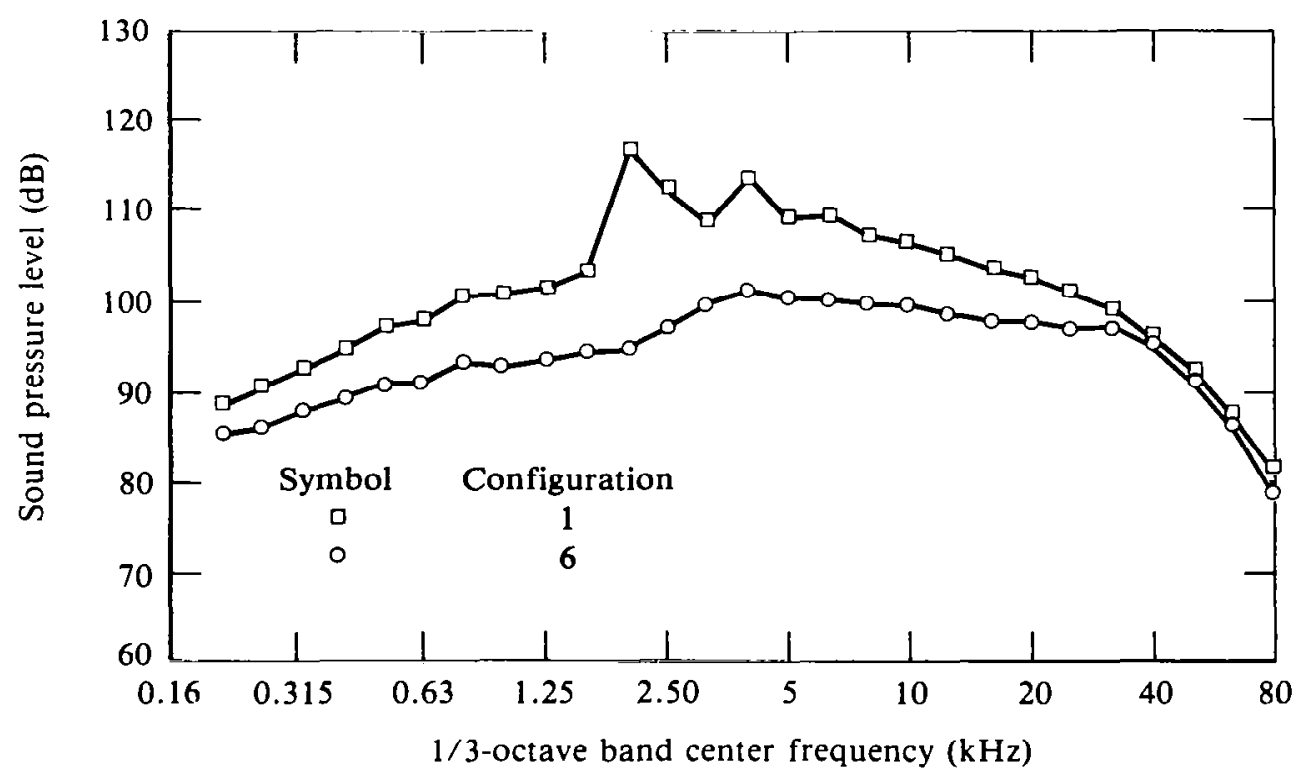

Figure A20. Sound pressure levels as a function of 1/3-octave band frequency; microphone 9 , pressure ratio $=2.8$, cold flow, and no forward motion. 


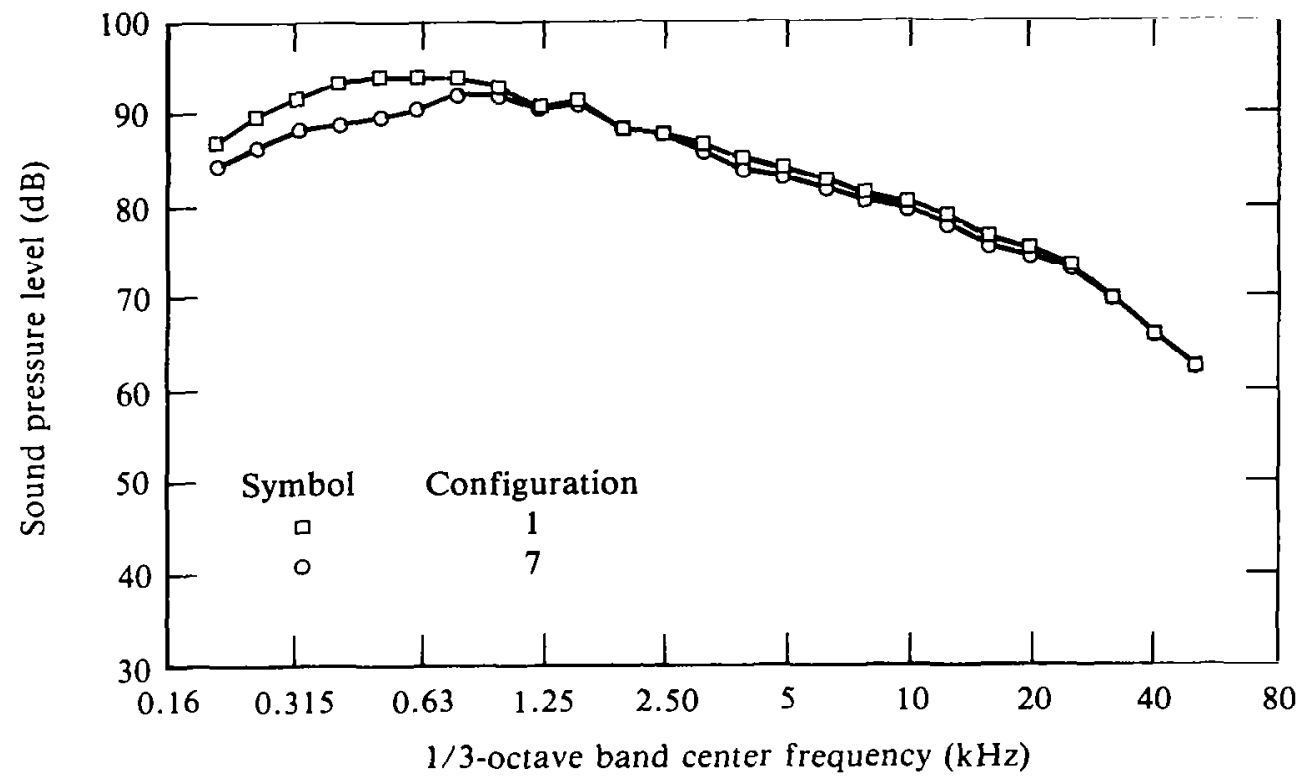

Figure A21. Sound pressure levels as a function of 1/3-octave band frequency; microphone 3 , pressure ratio $=1.6$, cold flow, and no forward motion.

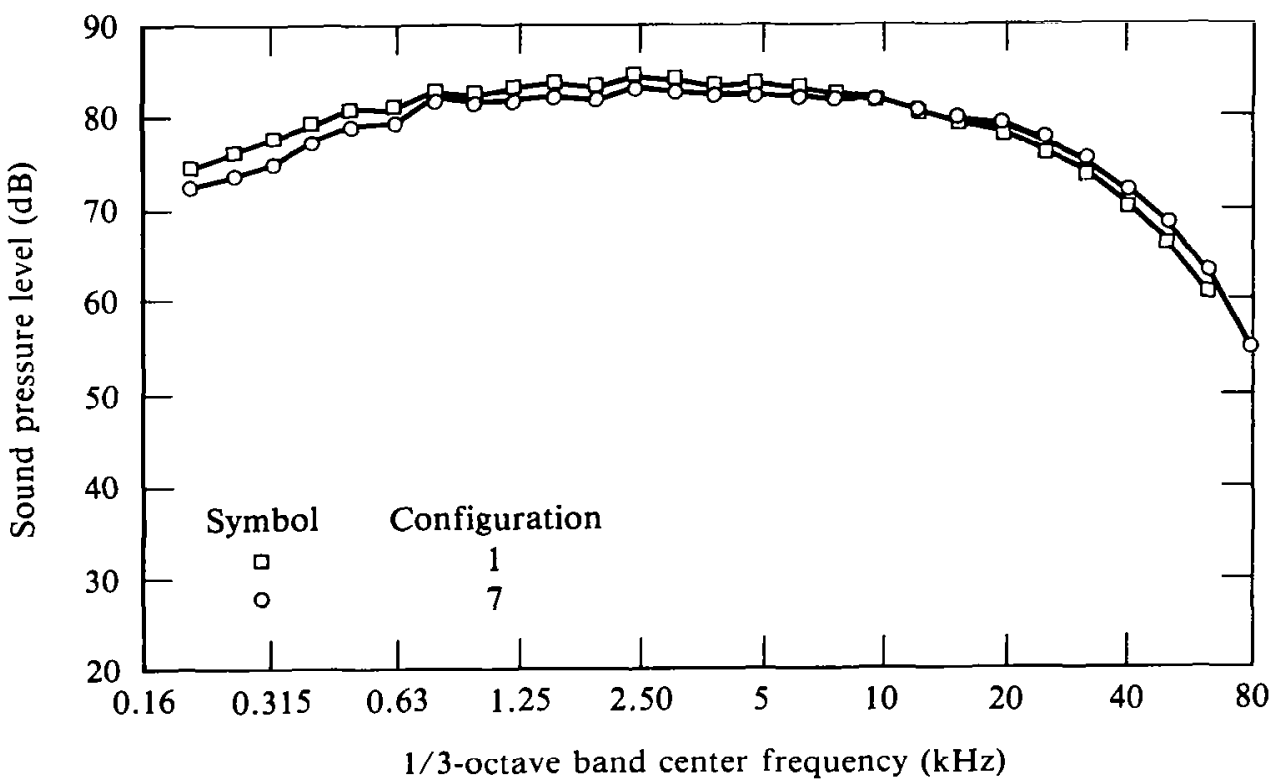

Figure A22. Sound pressure levels as a function of 1/3-octave band frequency; microphone 9 , pressure ratio $=1.6$, cold flow, and no forward motion. 


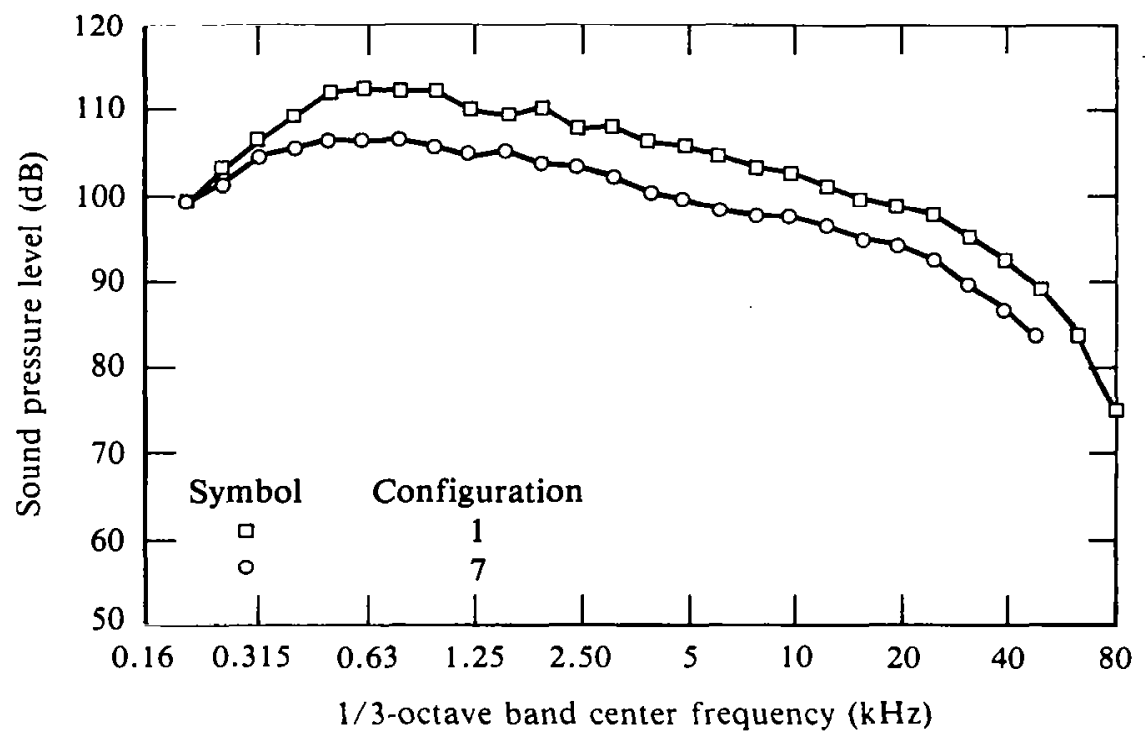

Figure A23. Sound pressure levels as a function of 1/3-octave band frequency; microphone 3 , pressure ratio $=2.8$, cold flow, and no forward motion.

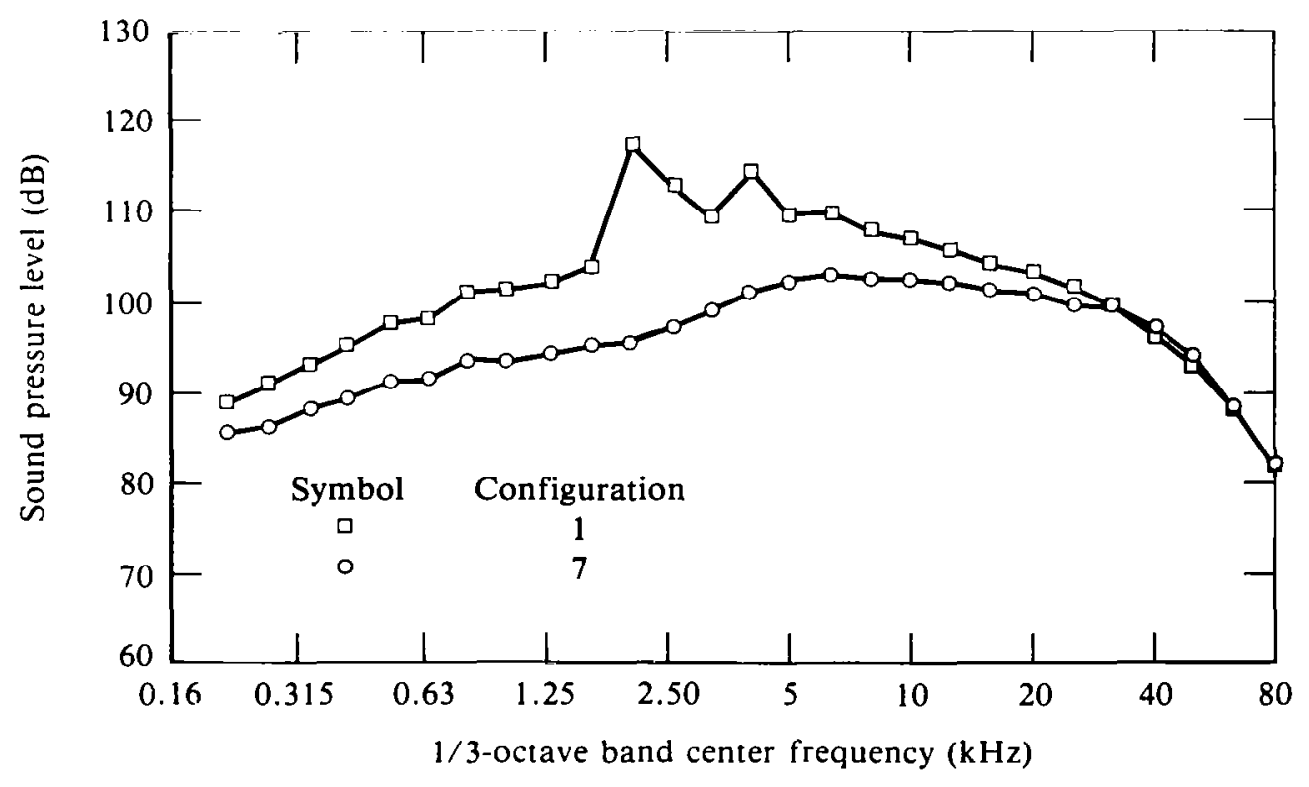

Figure A24. Sound pressure levels as a function of 1/3-octave band frequency; microphone 9 , pressure ratio $=\mathbf{2 . 8}$, cold flow, and no forward motion. 


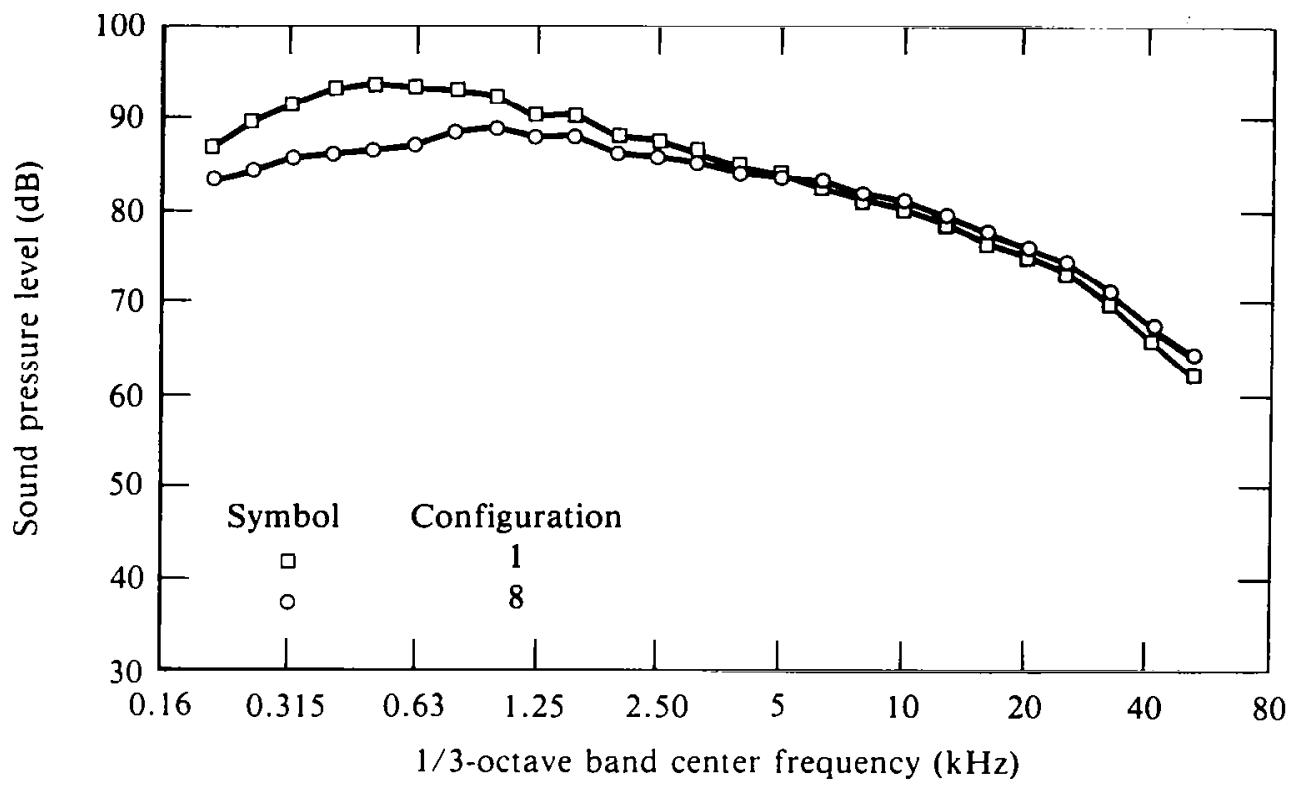

Figure A25. Sound pressure levels as a function of 1/3-octave band frequency; microphone 3 , pressure ratio $=1.6$, cold flow, and no forward motion.

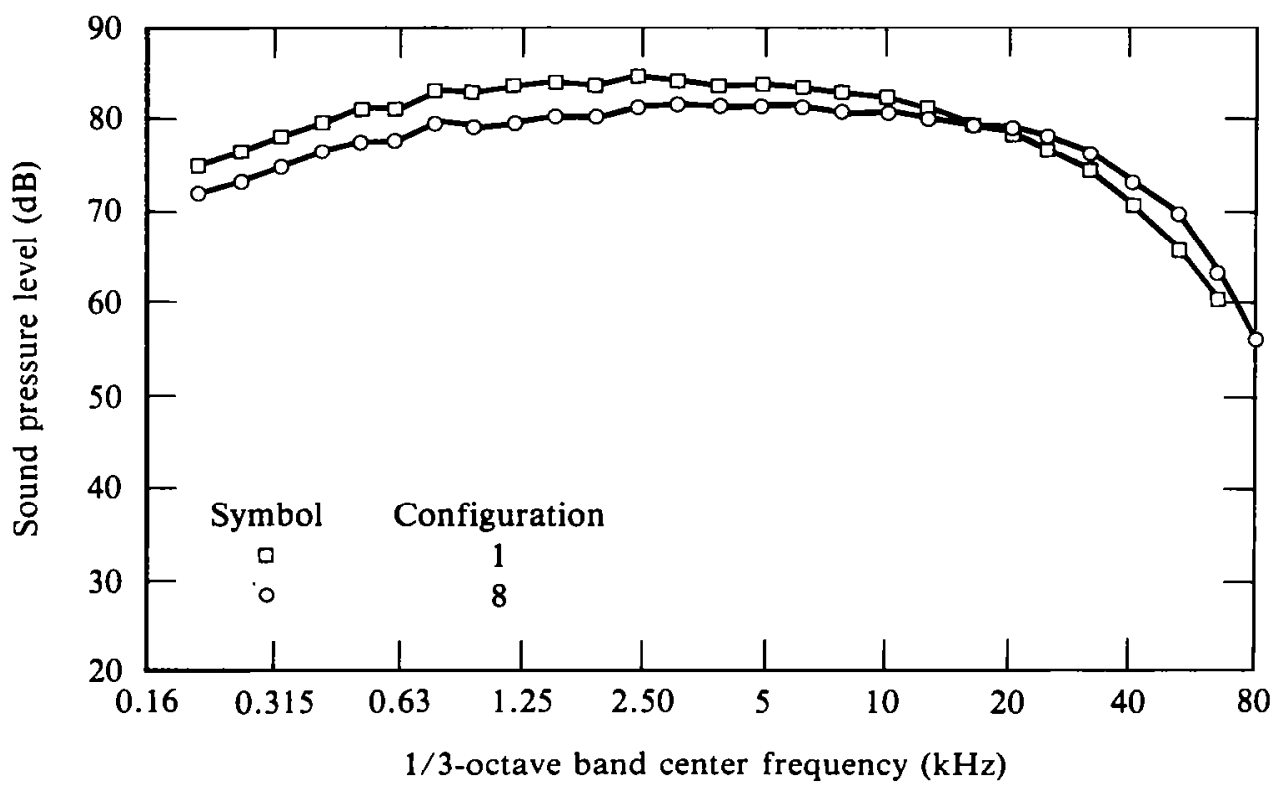

Figure A26. Sound pressure levels as a function of 1/3-octave band frequency; microphone 9 , pressure ratio $=1.6$, cold flow, and no forward motion. 


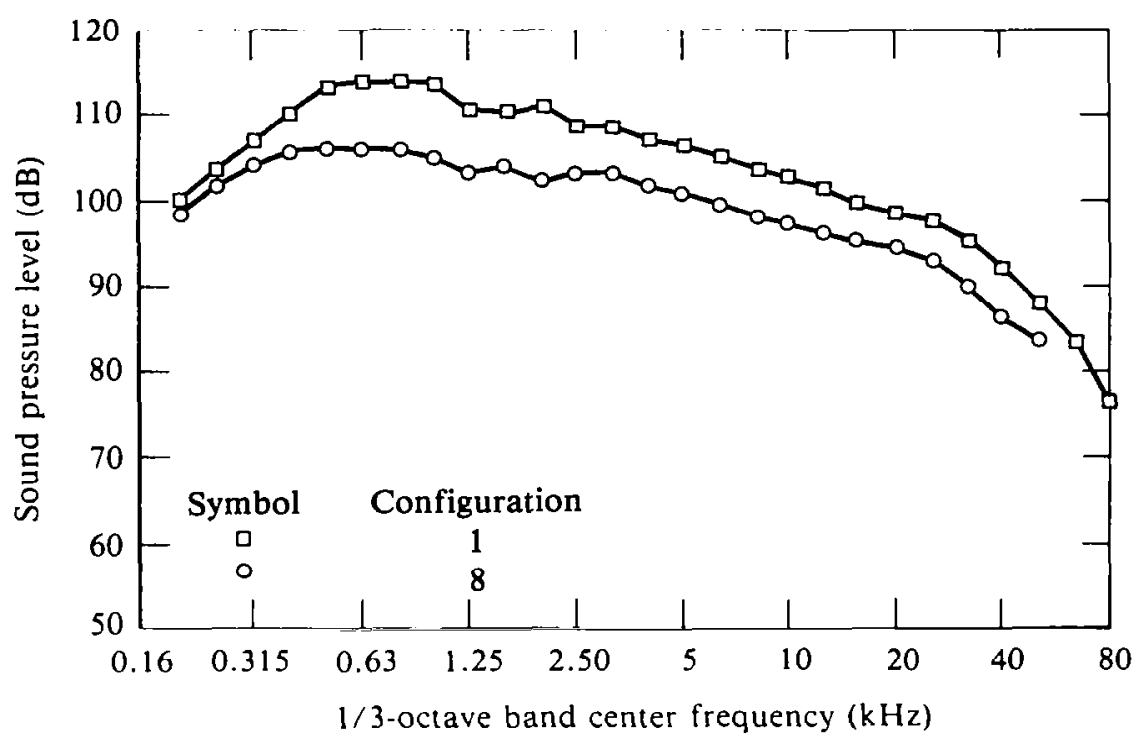

Figure A27. Sound pressure levels as a function of 1/3-octave band frequency; microphone 3 , pressure ratio $=\mathbf{2 . 8}$, cold flow, and no forward motion.

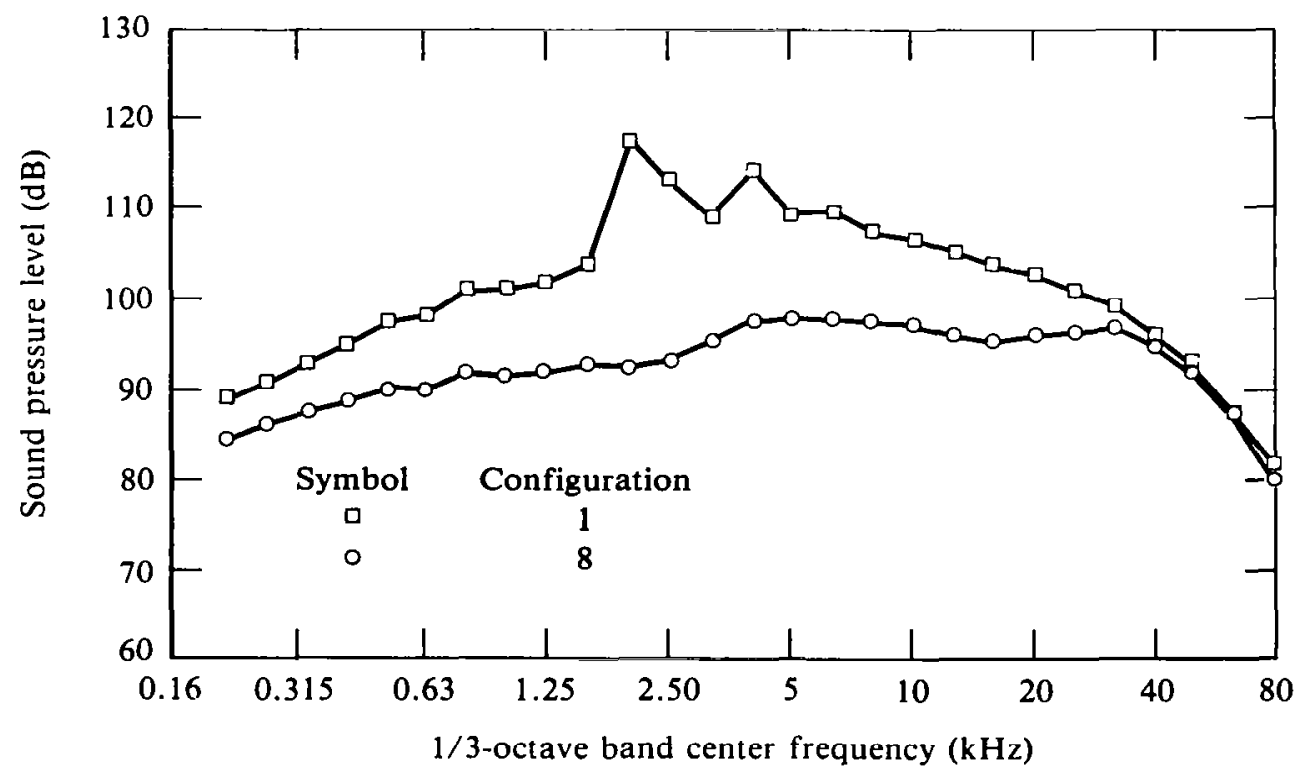

Figure A28. Sound pressure levels as a function of 1/3-octave band frequency; microphone 9 , pressure ratio $=2.8$, cold flow, and no forward motion. 


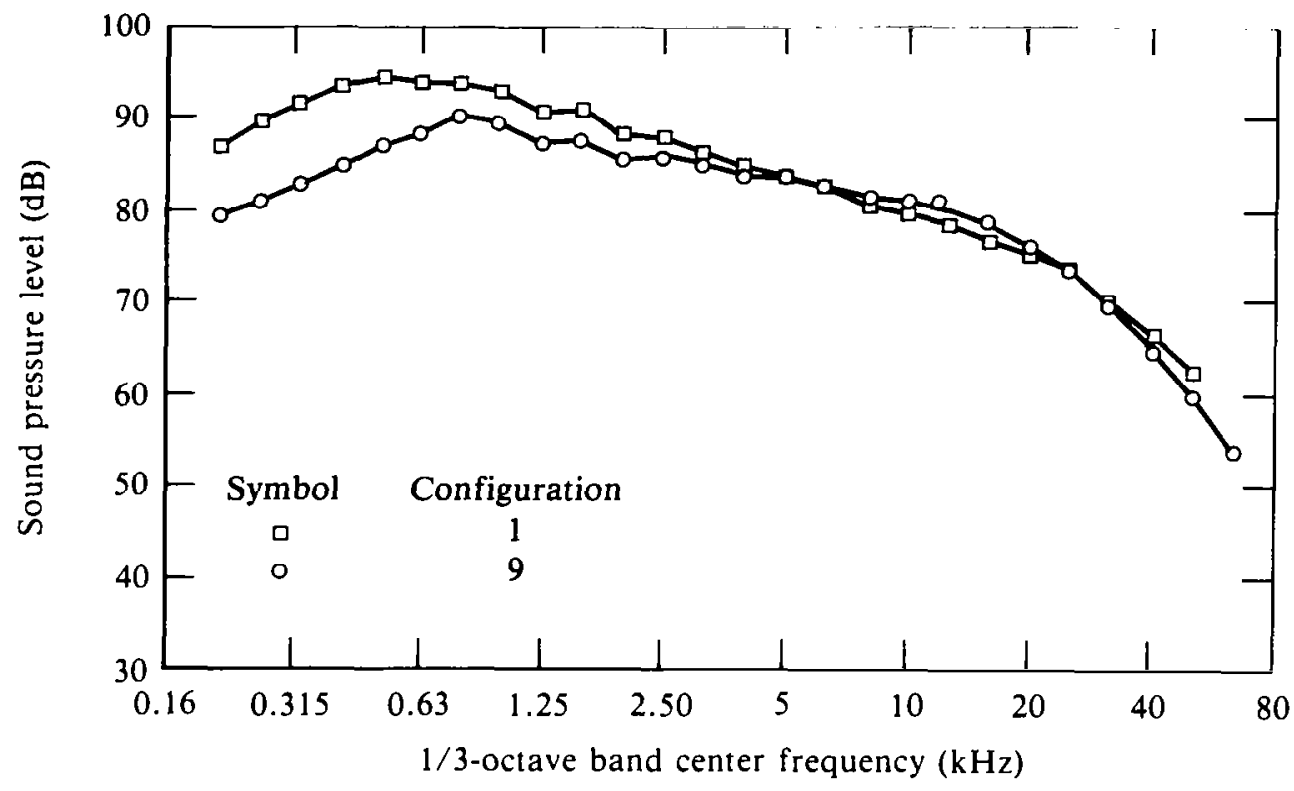

Figure A29. Sound pressure levels as a function of 1/3-octave band frequency; microphone 3 , pressure ratio $=1.6$, cold flow, and no forward motion.

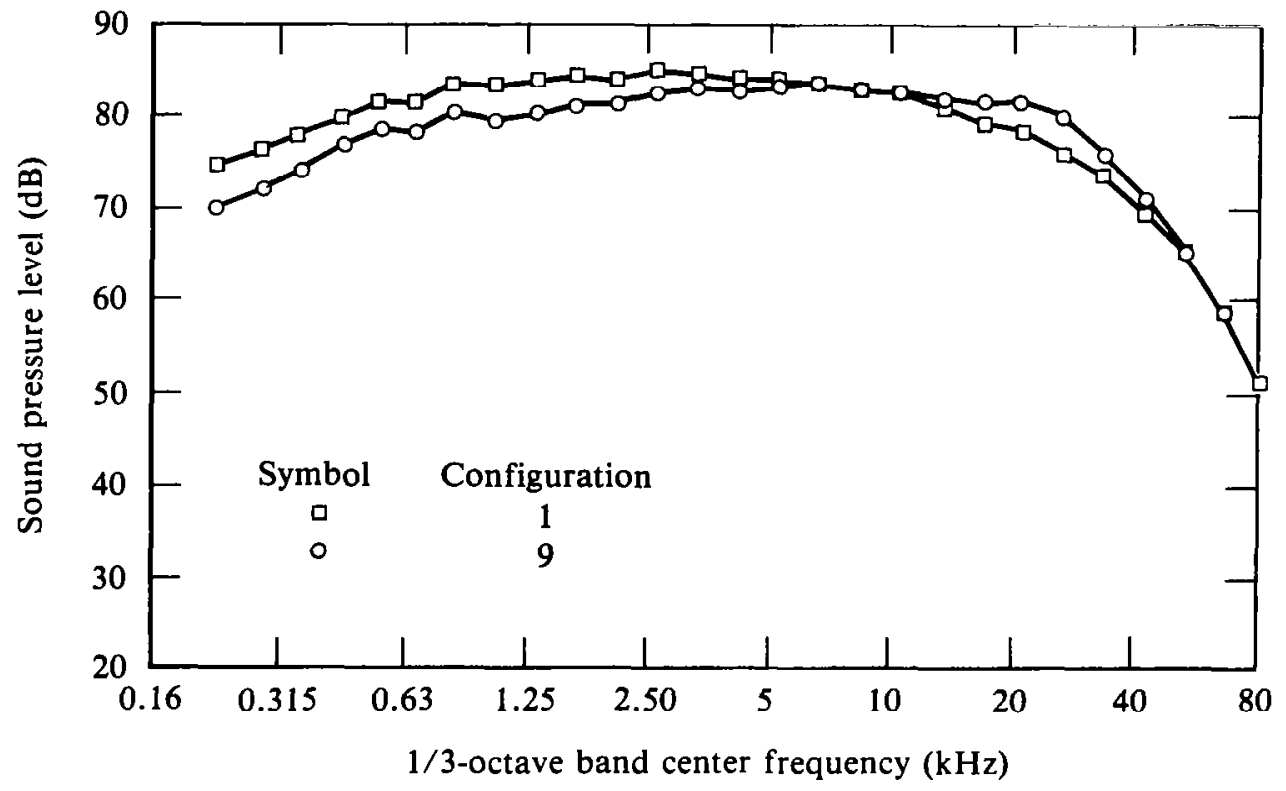

Figure A30. Sound pressure levels as a function of 1/3-octave band frequency; microphone 9 , pressure ratio $=1.6$, cold flow, and no forward motion. 


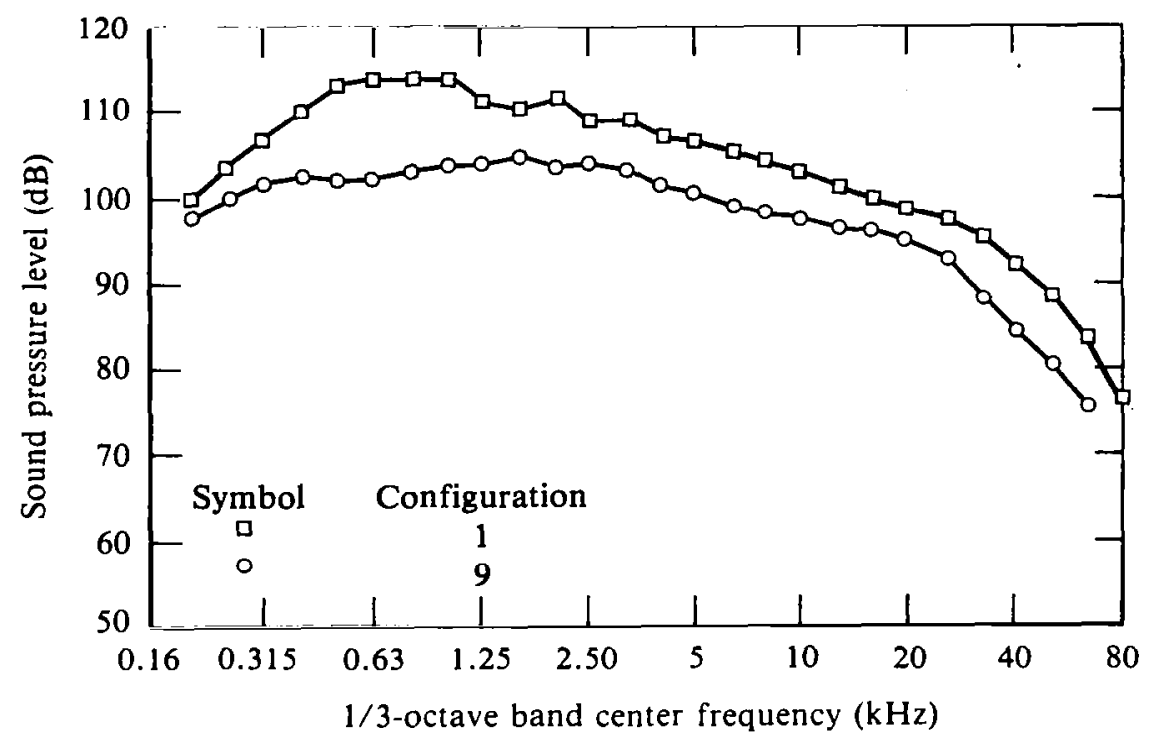

Figure A31. Sound pressure levels as a function of 1/3-octave band frequency; microphone 3 , pressure ratio $=2.8$, cold flow, and no forward motion.

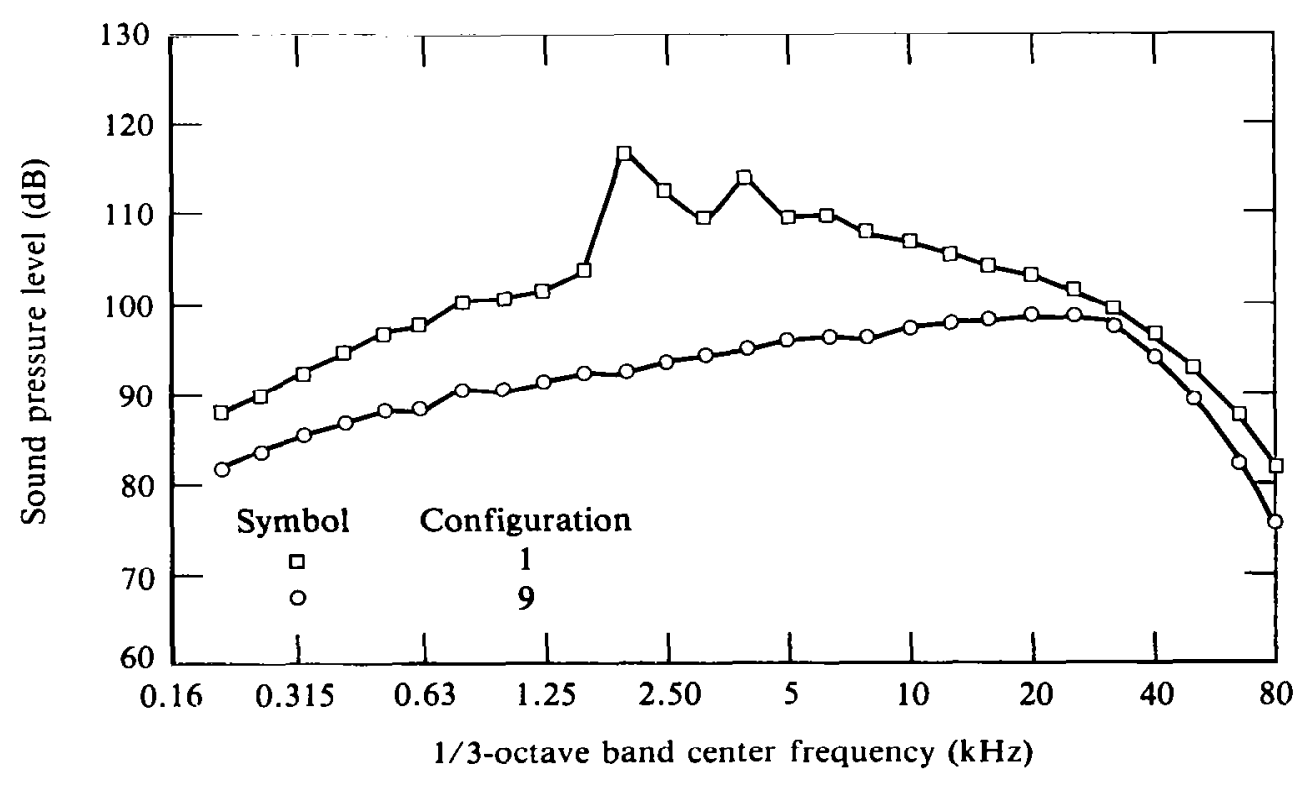

Figure A32. Sound pressure levels as a function of 1/3-octave band frequency; microphone 9 , pressure ratio $=2.8$, cold flow, and no forward motion. 


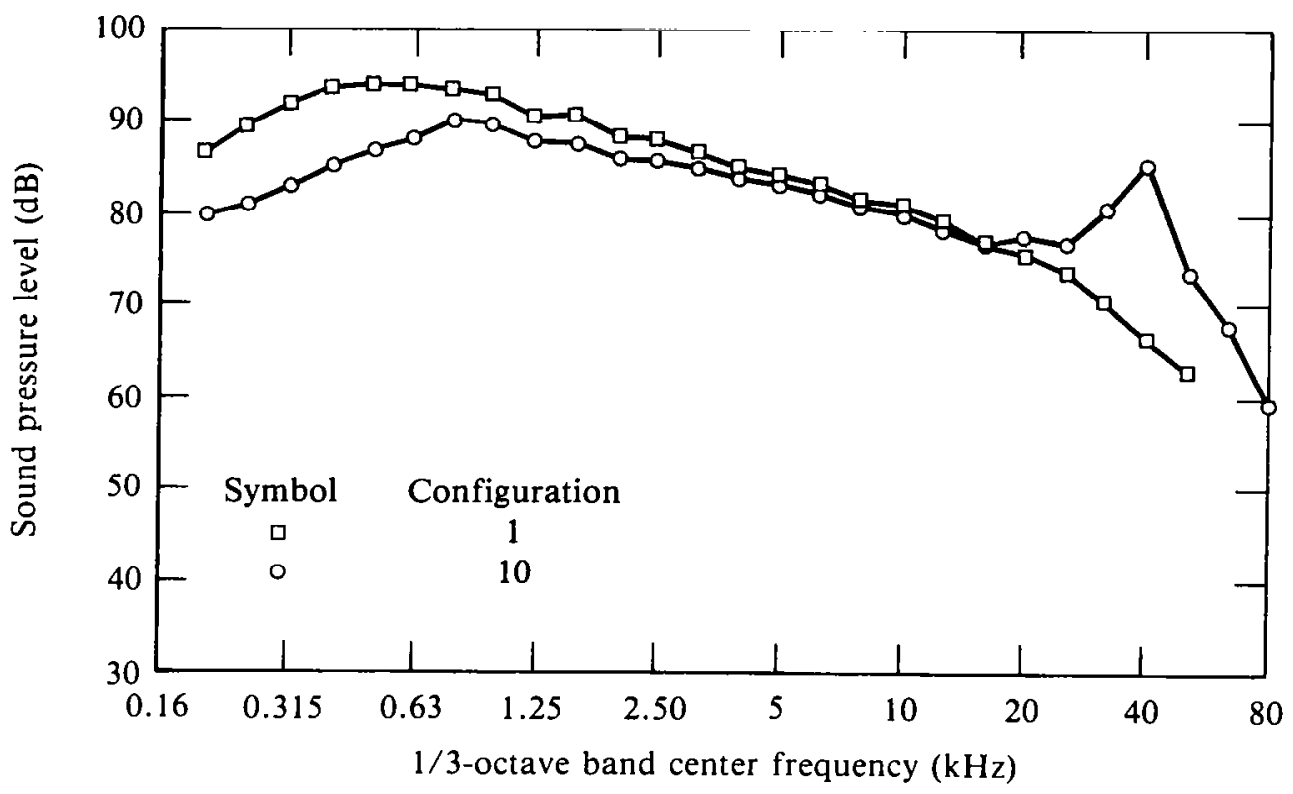

Figure A33. Sound pressure levels as a function of 1/3-octave band frequency; microphone 3 , pressure ratio $=1.6$, cold flow, and no forward motion.

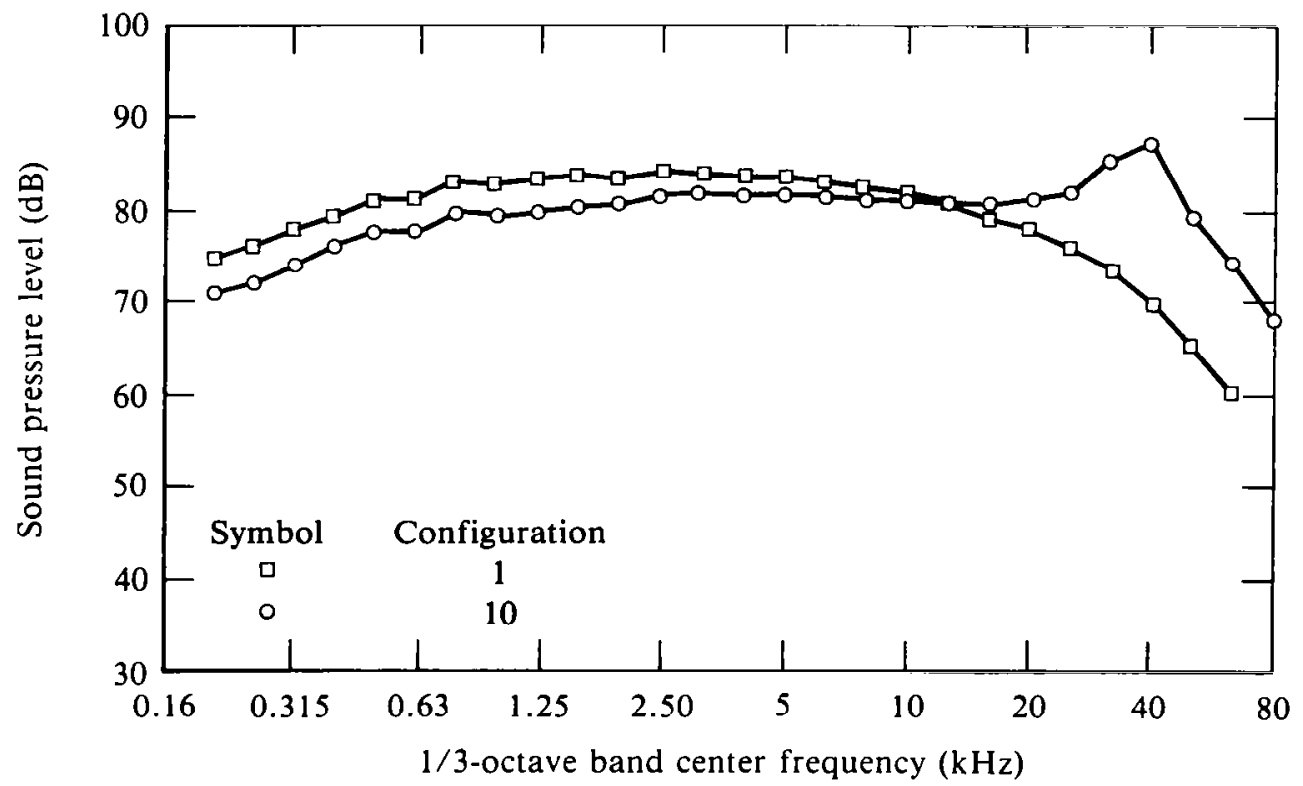

Figure A34. Sound pressure levels as a function of 1/3-octave band frequency; microphone 9 , pressure ratio $=1.6$, cold flow, and no forward motion. 


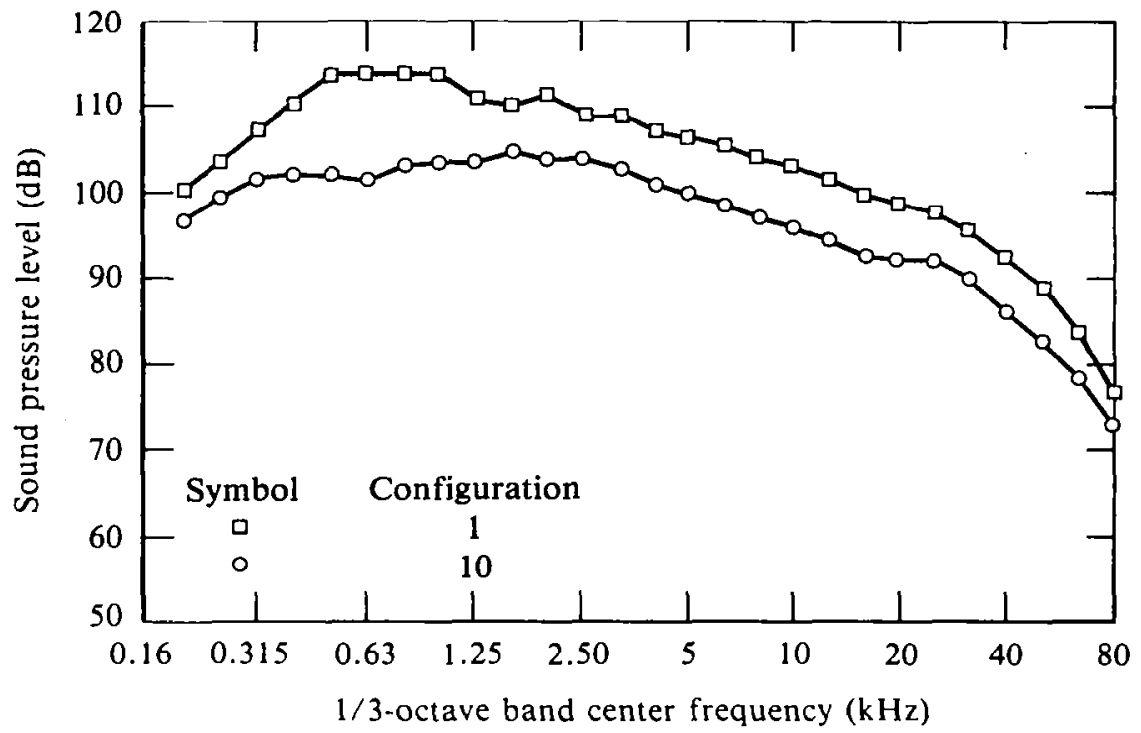

Figure A35. Sound pressure levels as a function of 1/3-octave band frequency; microphone 3 , pressure ratio $=2.8$, cold flow, and no forward motion.

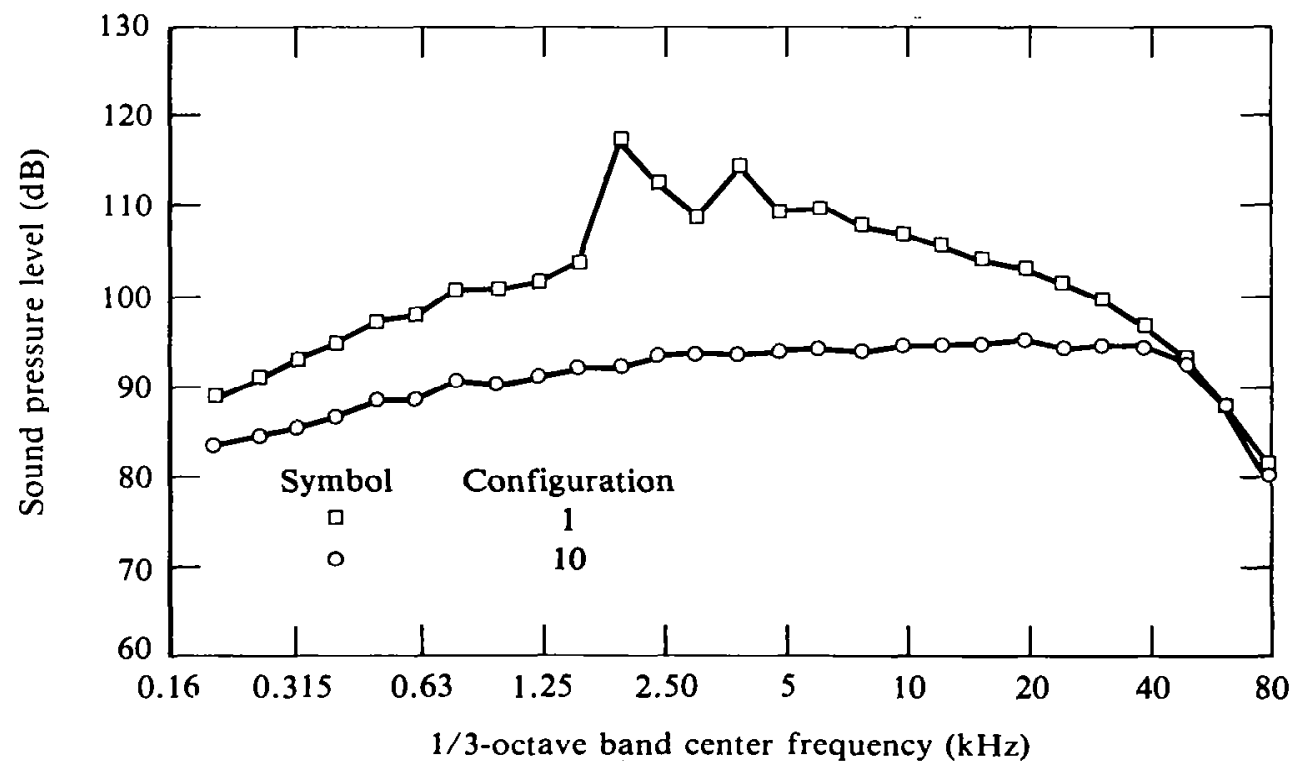

Figure A36. Sound pressure levels as a function of 1/3-octave band frequency; microphone 9 , pressure ratio $=2.8$, cold flow, and no forward motion. 


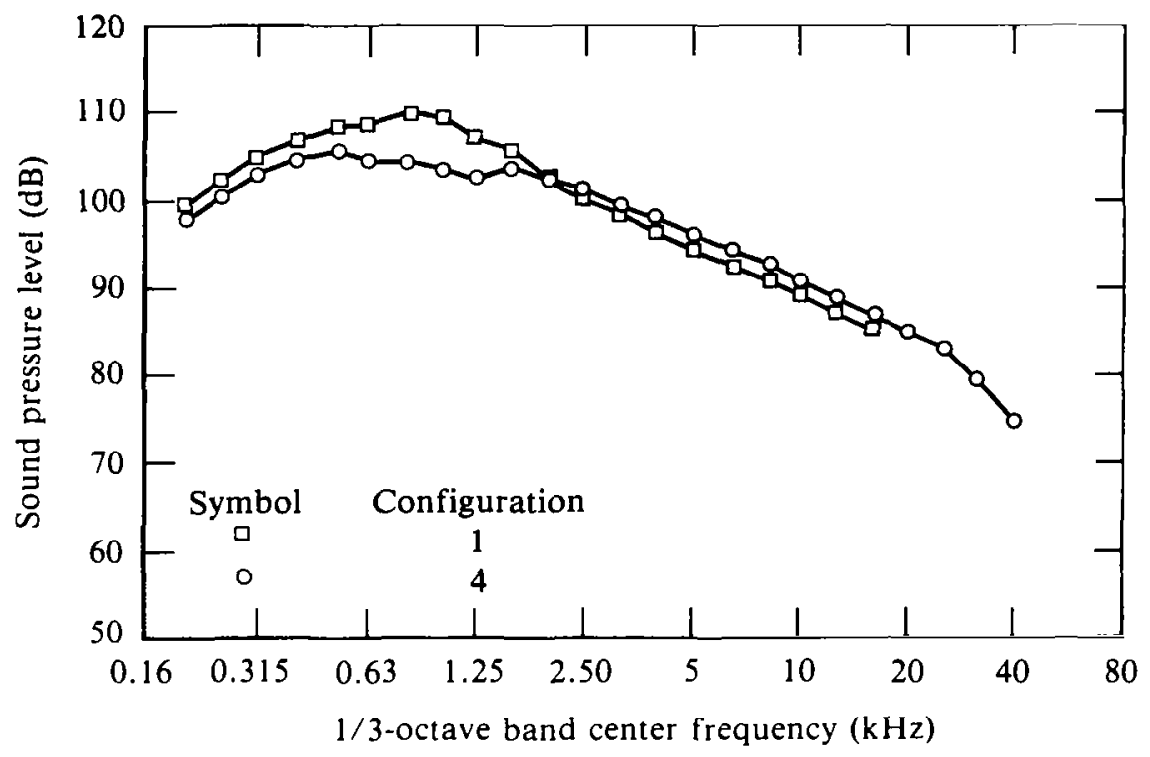

Figure A37. Sound pressure levels as a function of 1/3-octave band frequency; microphone 3 , pressure ratio $=1.6$, hot flow $\left(370^{\circ} \mathrm{C}\right)$, and no forward motion.

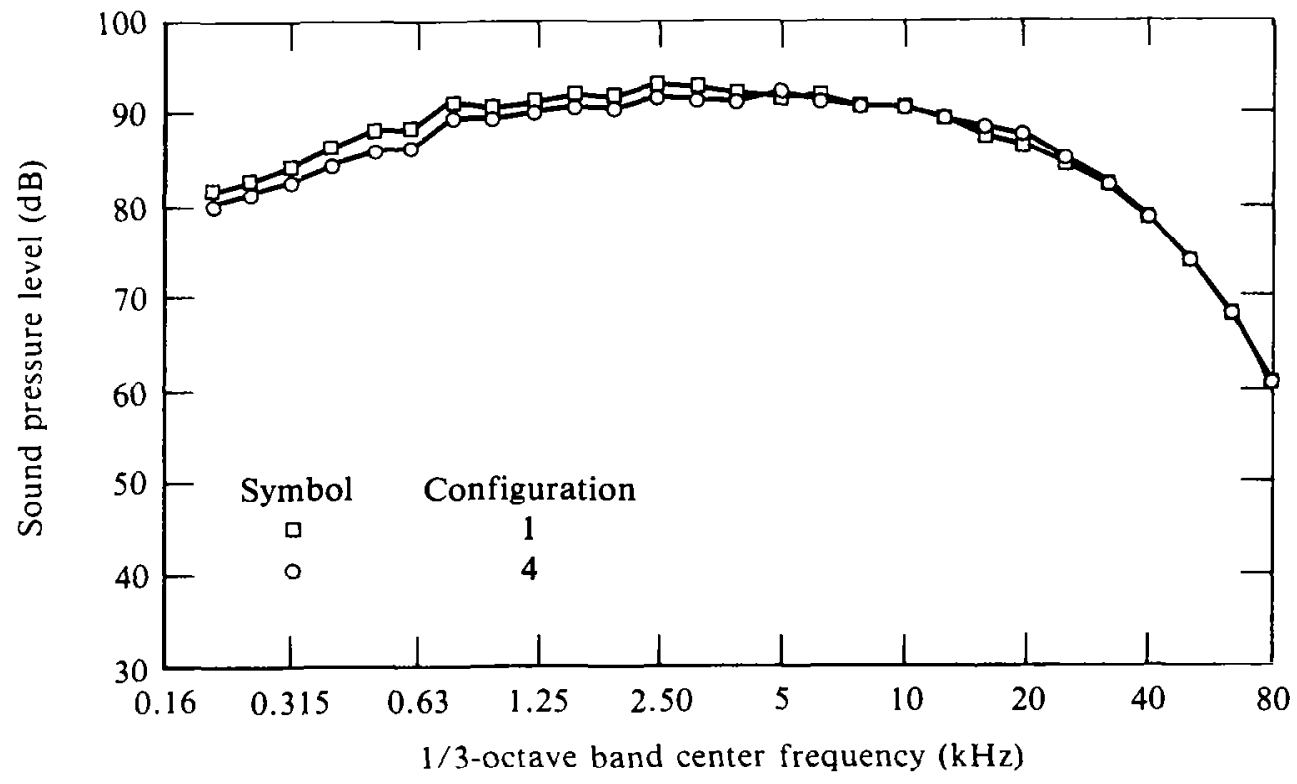

Figure A38. Sound pressure levels as a function of 1/3-octave band frequency; microphone 9 , pressure ratio $=1.6$, hot flow $\left(370^{\circ} \mathrm{C}\right)$, and no forward motion. 


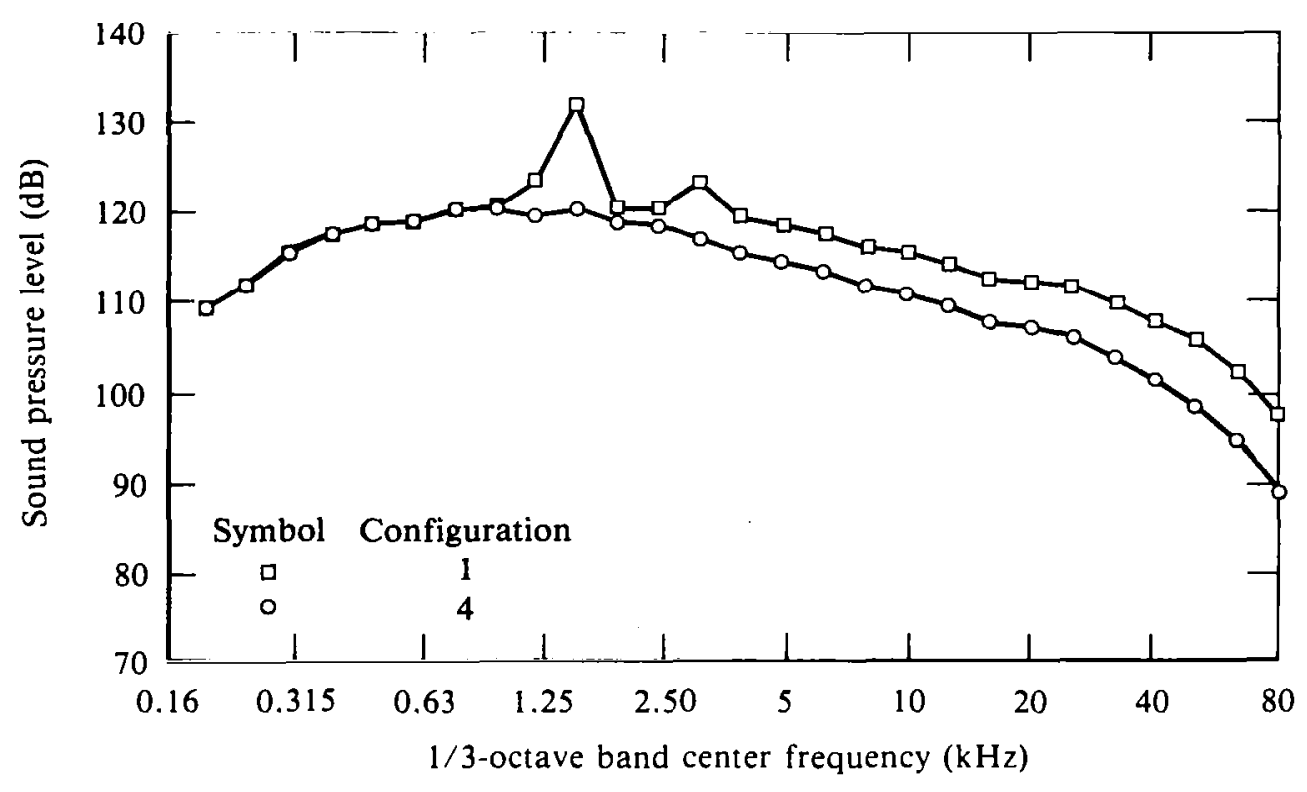

Figure A39. Sound pressure levels as a function of 1/3-octave band frequency; microphone 3 , pressure ratio $=2.8$, hot flow $\left(370^{\circ} \mathrm{C}\right)$, and no forward motion.

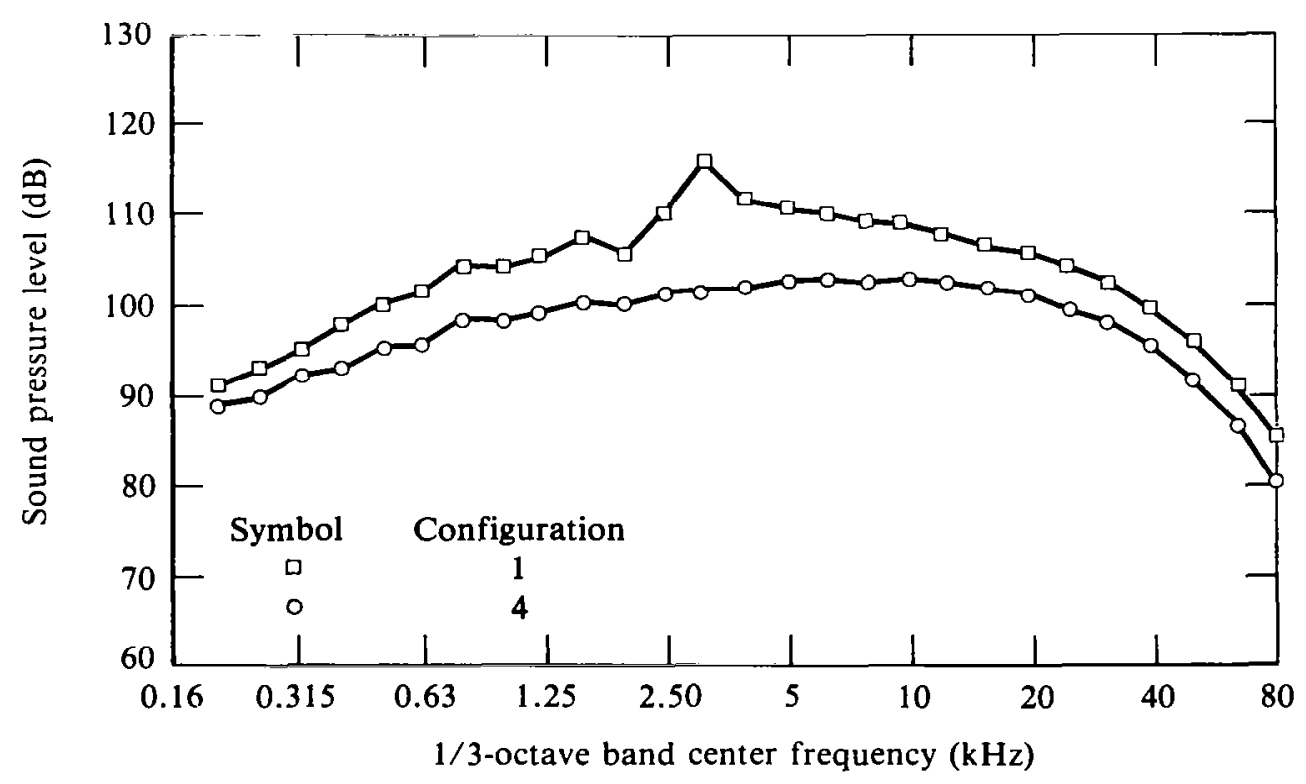

Figure A40. Sound pressure levels as a function of 1/3-octave band frequency; microphone 9 , pressure ratio $=2.8$, hot flow $\left(370^{\circ} \mathrm{C}\right)$, and no forward motion. 


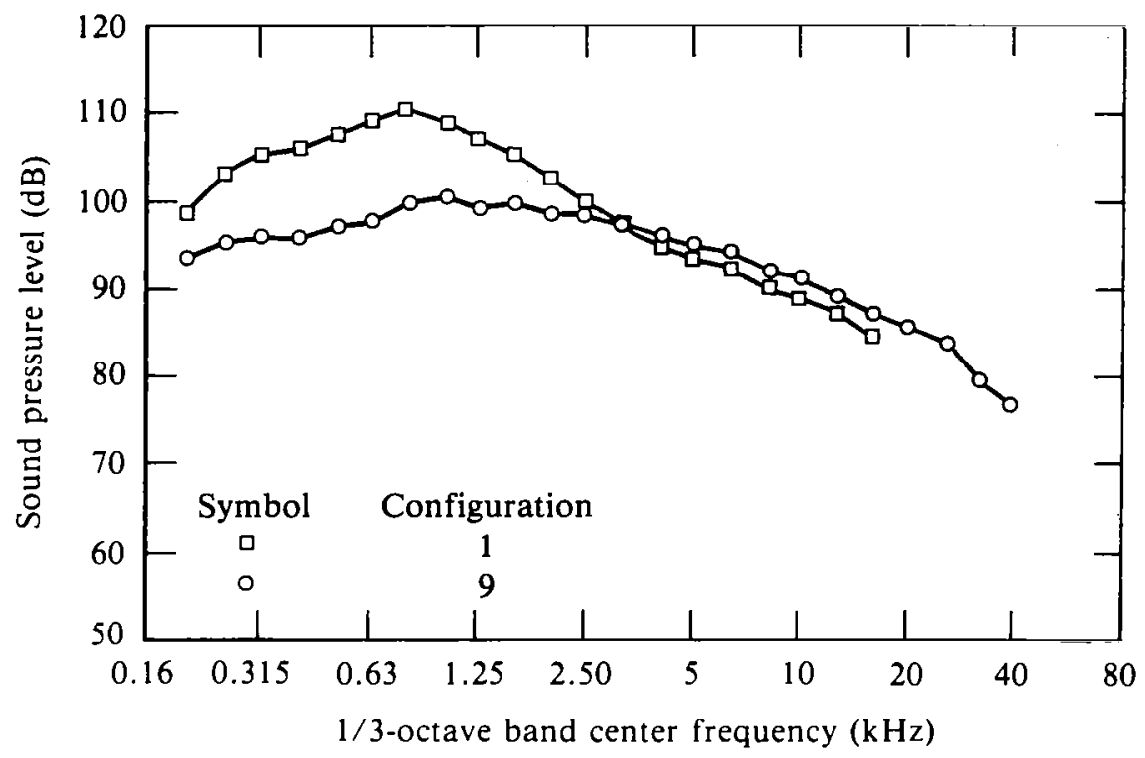

Figure A41. Sound pressure levels as a function of 1/3-octave band frequency; microphone 3 , pressure ratio $=1.6$, hot flow $\left(370^{\circ} \mathrm{C}\right)$, and no forward motion.

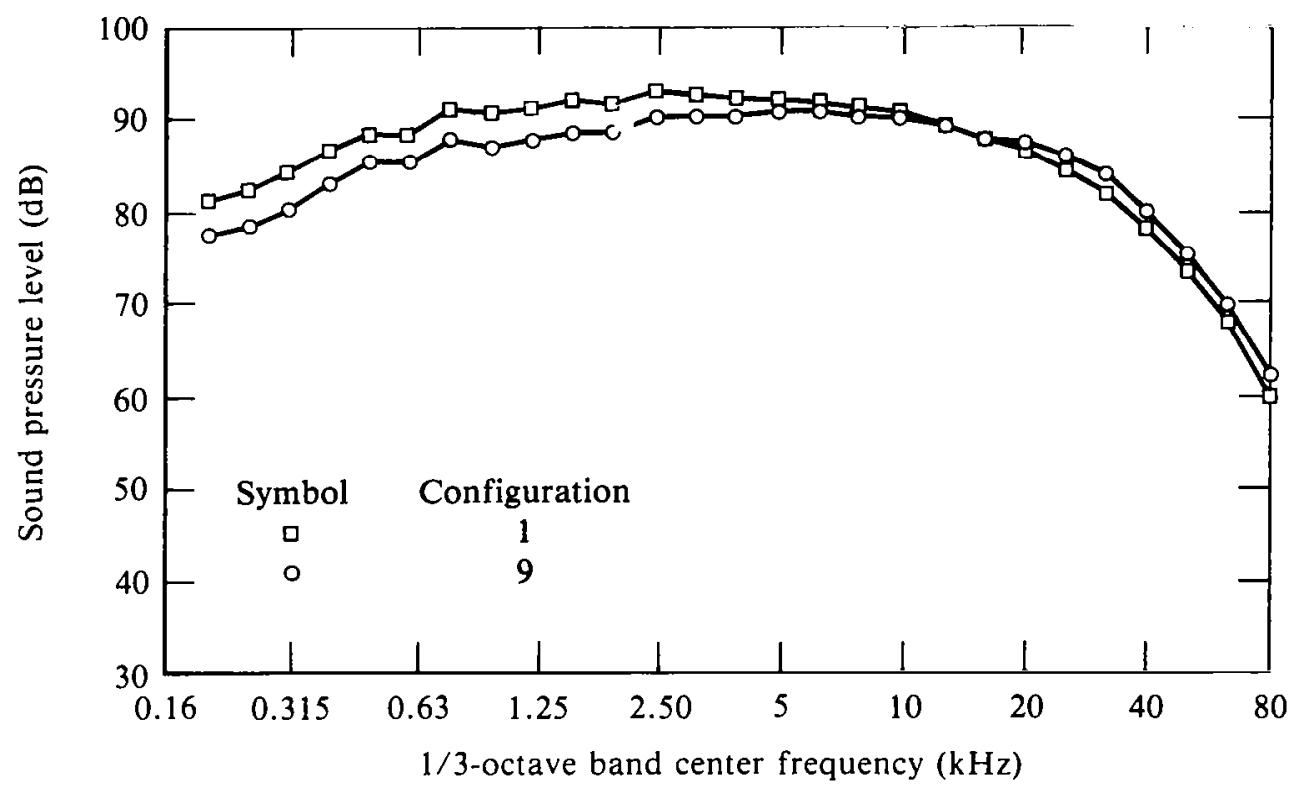

Figure A42. Sound pressure levels as a function of 1/3-octave band frequency; microphone 3 , pressure ratio $=1.6$, hot flow $\left(370^{\circ} \mathrm{C}\right)$, and no forward motion. 


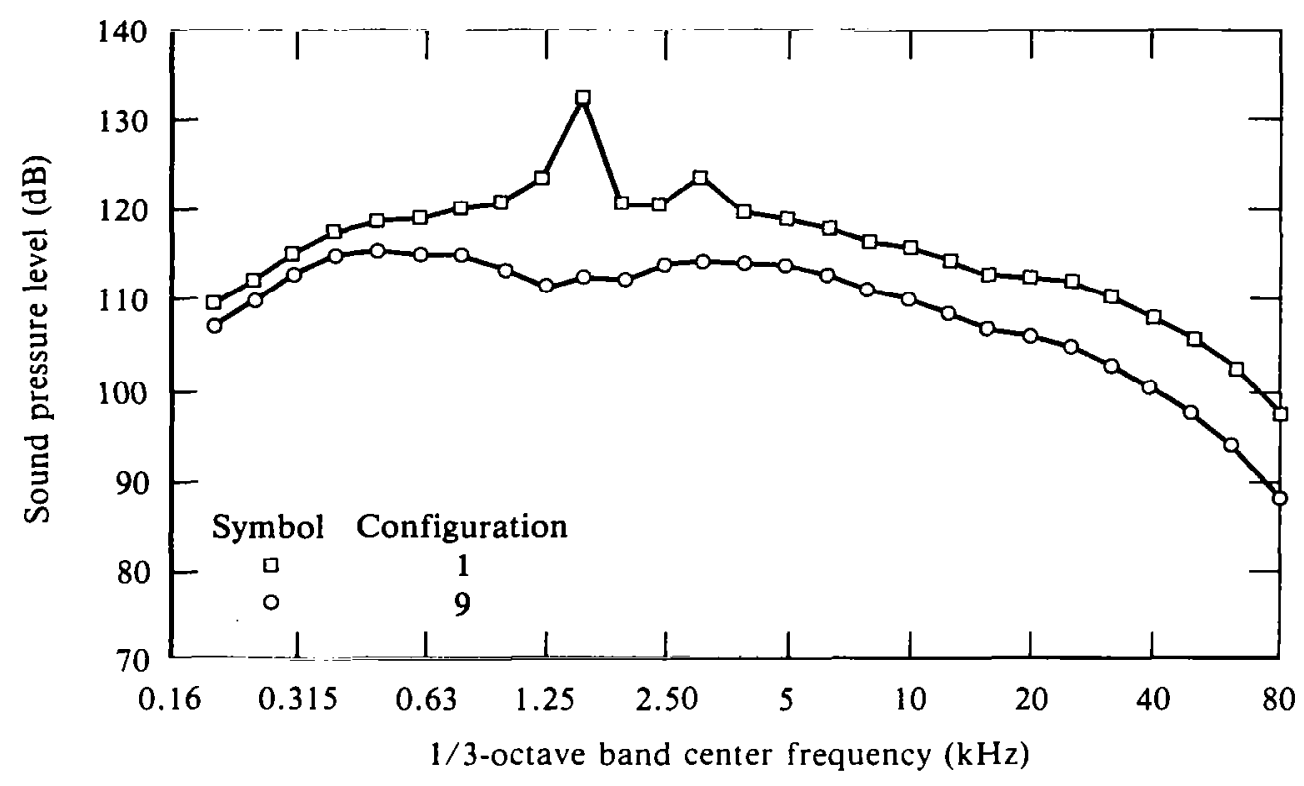

Figure A43. Sound pressure levels as a function of 1/3-octave band frequency; microphone 3 , pressure ratio $=2.8$, hot flow $\left(370^{\circ} \mathrm{C}\right)$, and no forward motion.

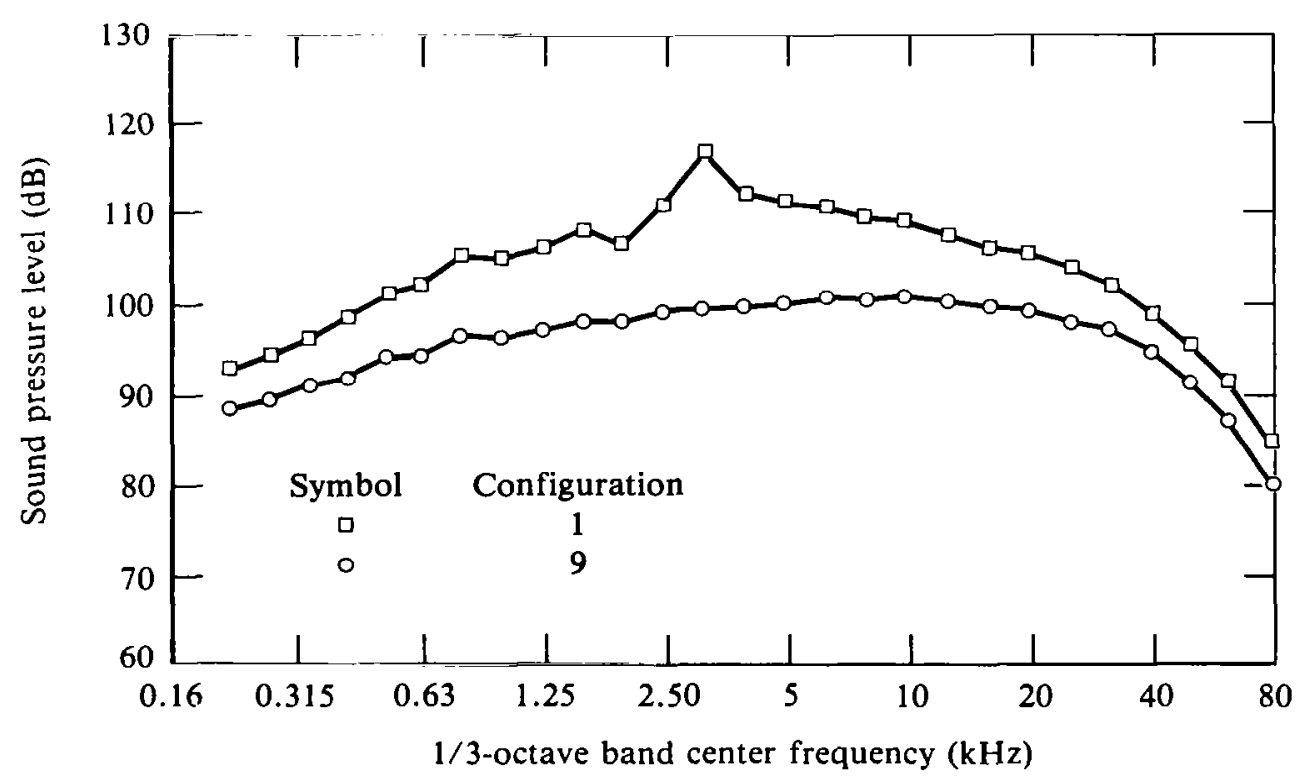

Figure A44. Sound pressure levels as a function of 1/3-octave band frequency; microphone 9 , pressure ratio $=2.8$, hot flow $\left(370^{\circ} \mathrm{C}\right)$, and no forward motion. 


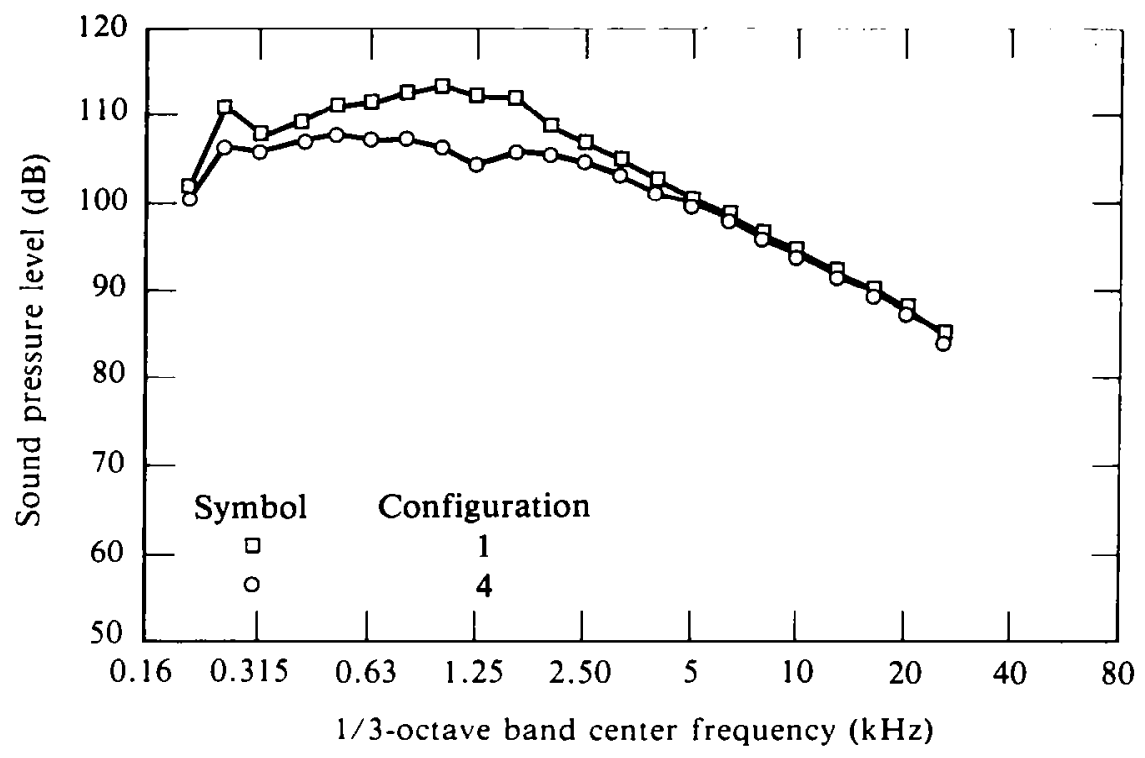

Figure A45. Sound pressure levels as a function of 1/3-octave band frequency; microphone 3 , pressure ratio $=1.6$, hot flow $\left(540^{\circ} \mathrm{C}\right)$, and no forward motion.

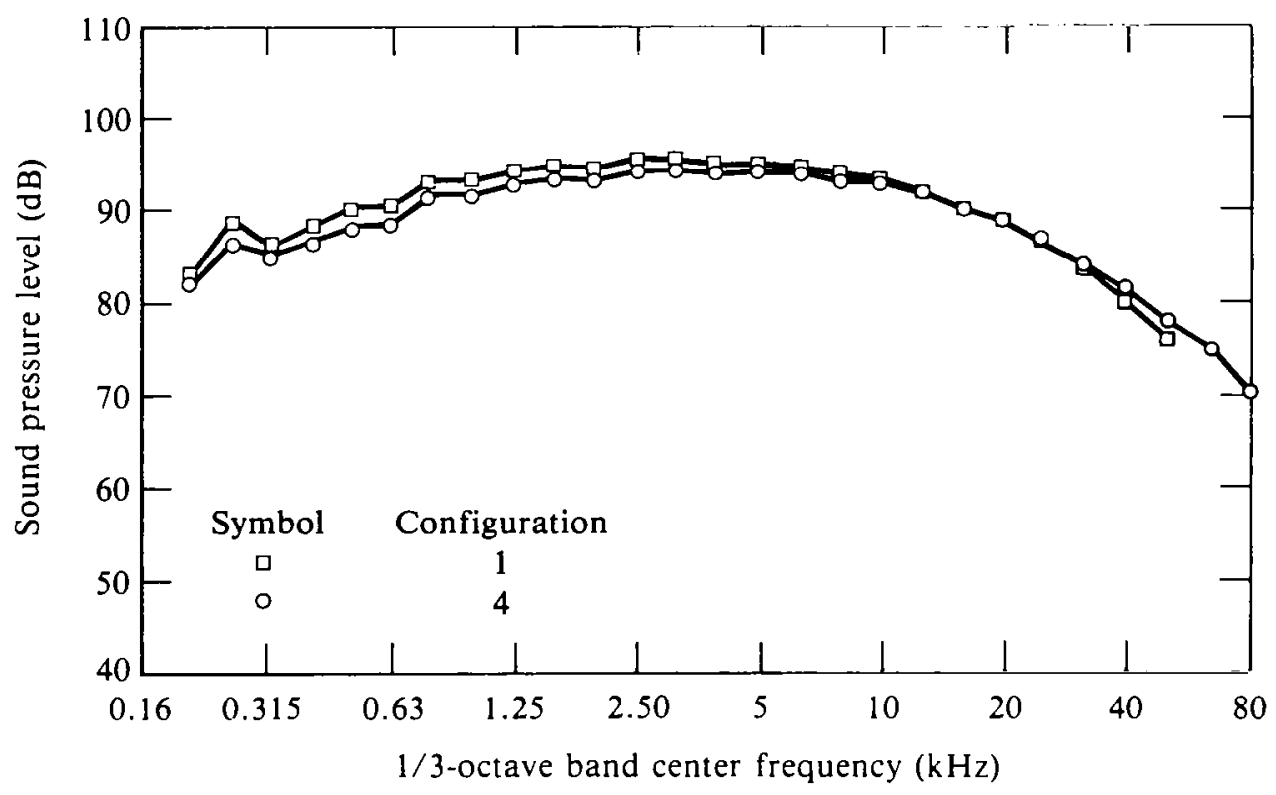

Figure A46. Sound pressure levels as a function of 1/3-octave band frequency; microphone 9 , pressure ratio $=1.6$, hot flow $\left(540^{\circ} \mathrm{C}\right)$, and no forward motion. 


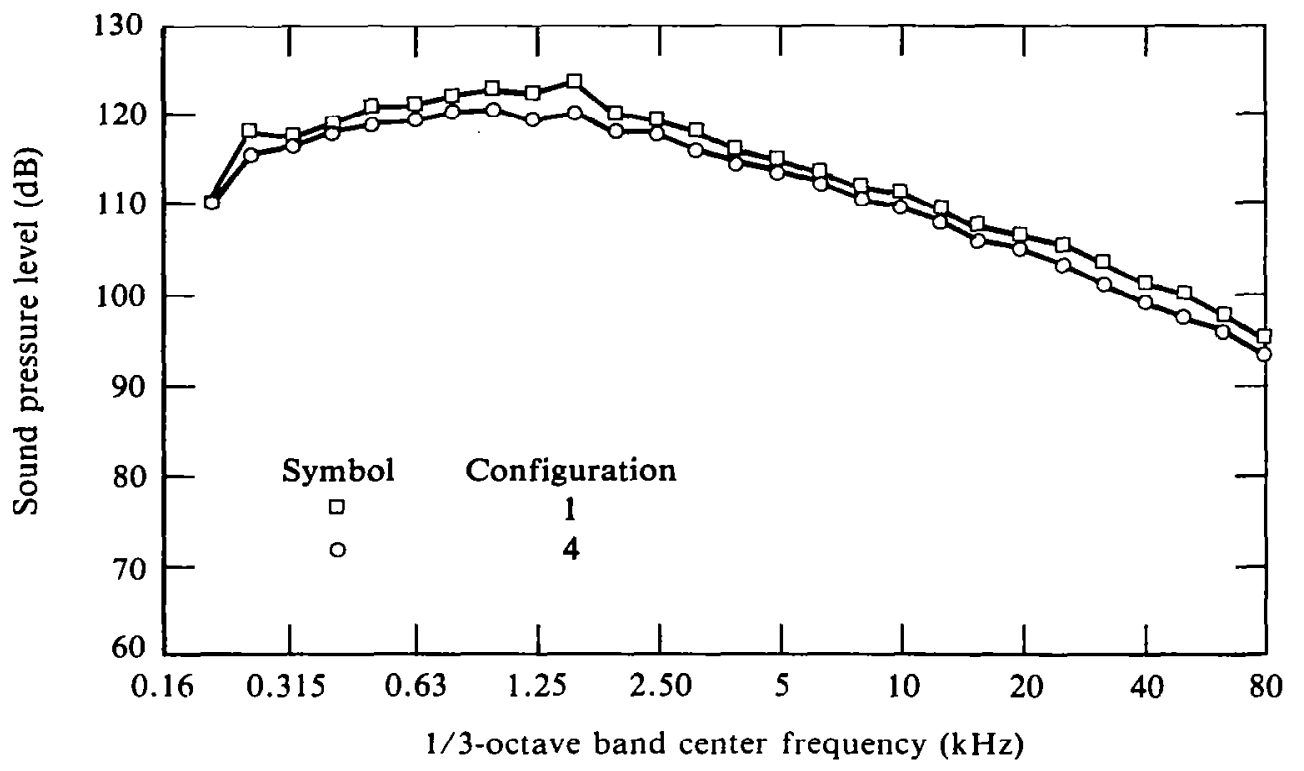

Figure A47. Sound pressure levels as a function of 1/3-octave band frequency; microphone 3 , pressure ratio $=2.8$, hot flow $\left(540^{\circ} \mathrm{C}\right)$, and no forward motion.

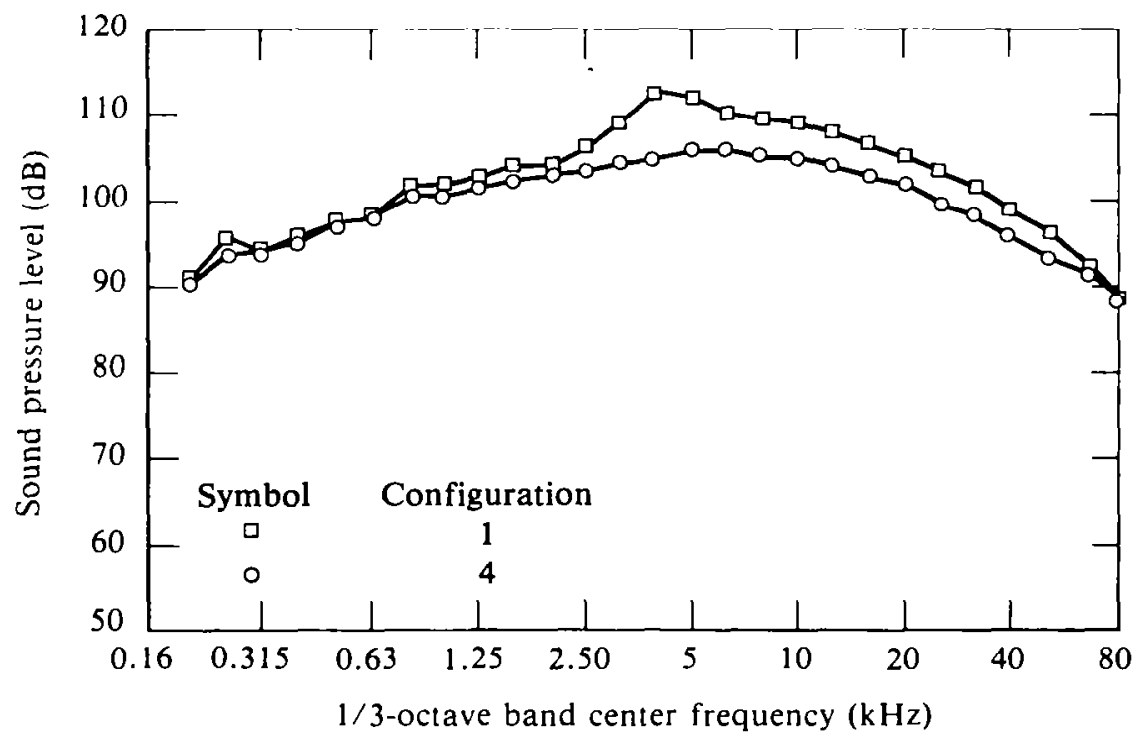

Figure A48. Sound pressure levels as a function of $1 / 3$-octave band frequency; microphone 9 , pressure ratio $=2.8$, hot flow $\left(540^{\circ} \mathrm{C}\right)$, and no forward motion. 


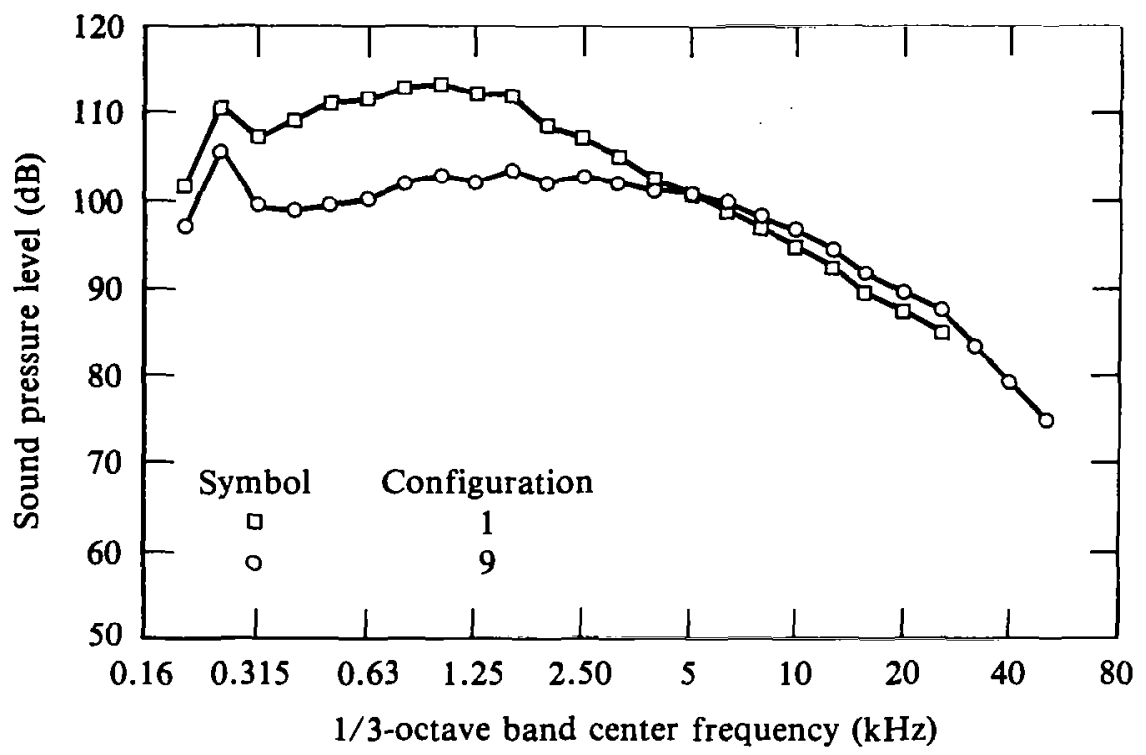

Figure A49. Sound pressure levels as a function of 1/3-octave band frequency; microphone 3 , pressure ratio $=1.6$, hot flow $\left(540^{\circ} \mathrm{C}\right)$, and no forward motion.

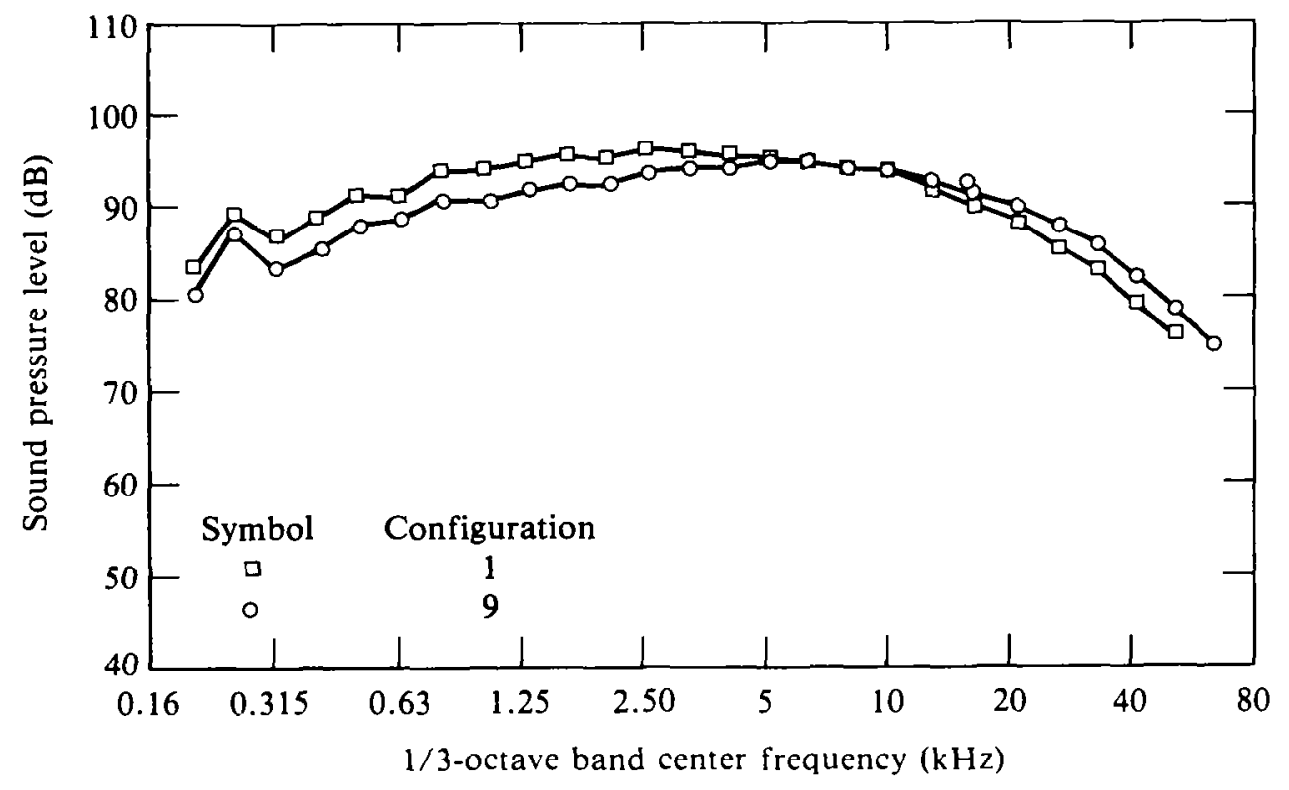

Figure A50. Sound pressure levels as a function of 1/3-octave band frequency; microphone 9 , pressure ratio $=1.6$, hot flow $\left(540^{\circ} \mathrm{C}\right)$, and no forward motion. 


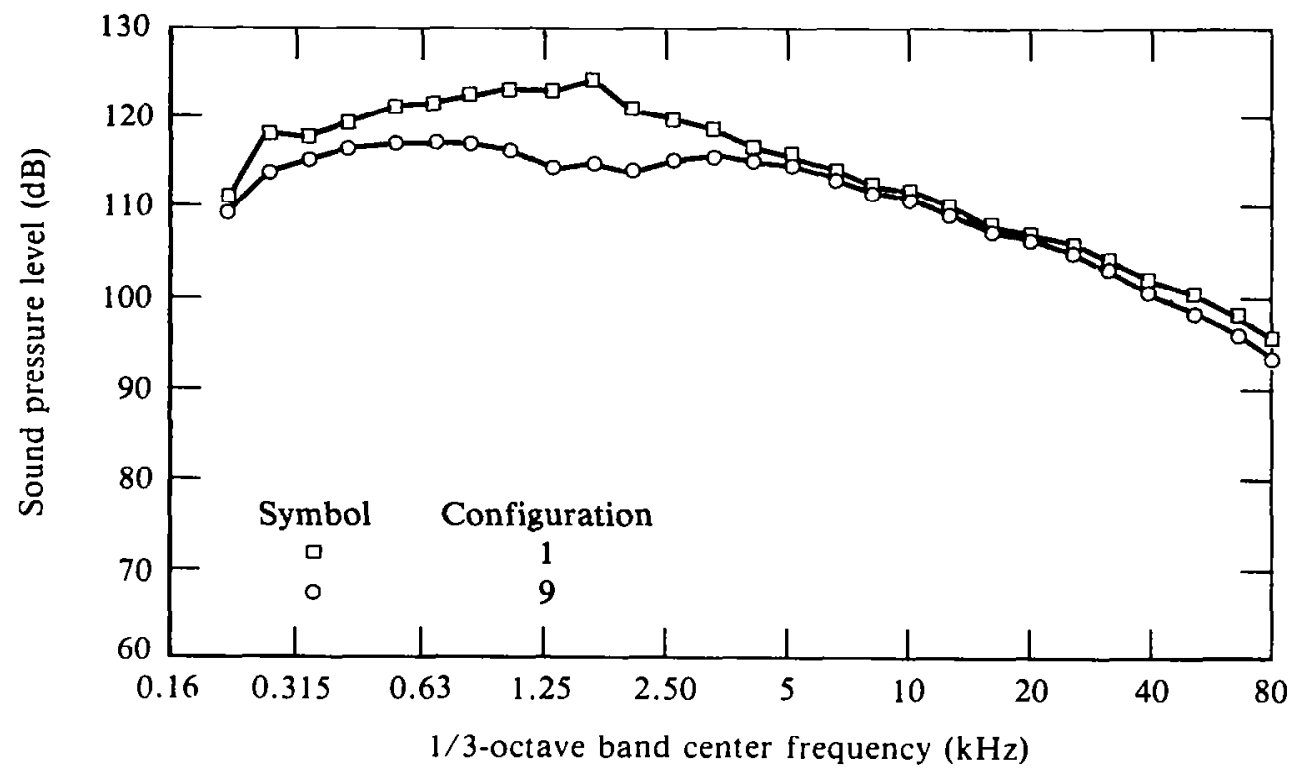

Figure A51. Sound pressure levels as a function of 1/3-octave band frequency; microphone 3 , pressure ratio $=2.8$, hot flow $\left(540^{\circ} \mathrm{C}\right)$, and no forward motion.

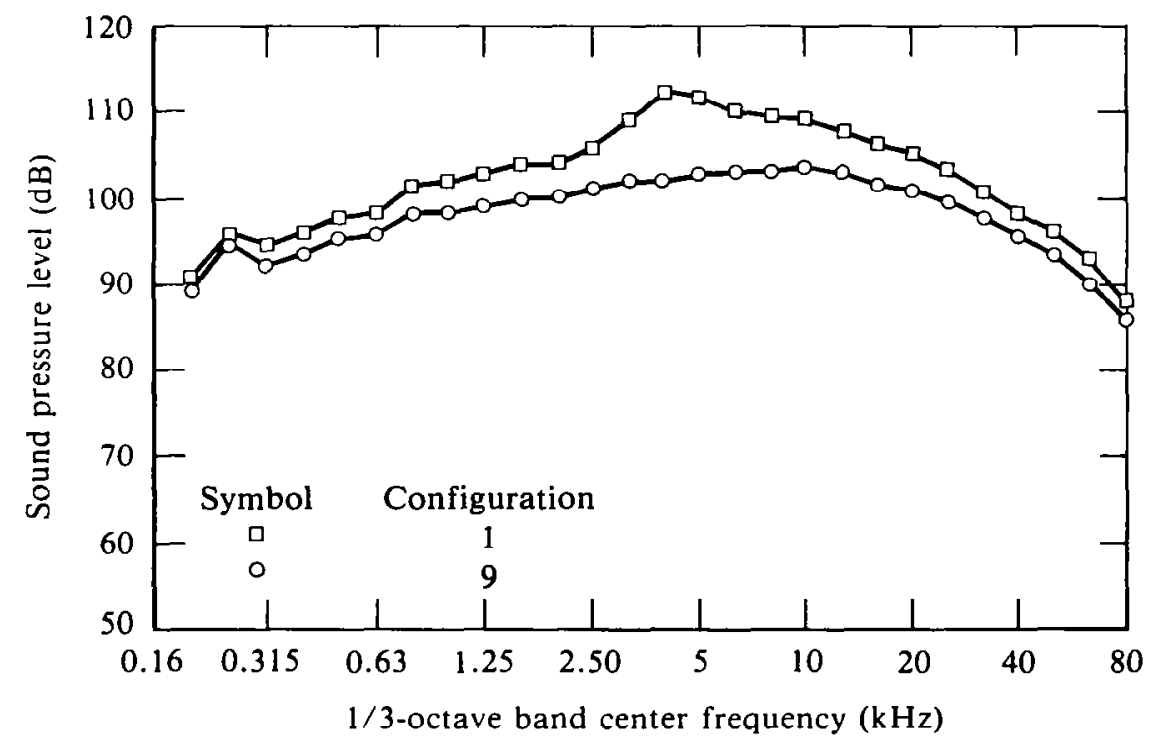

Figure A52. Sound pressure levels as a function of 1/3-octave band frequency; microphone 9 , pressure ratio $=2.8$, hot flow $\left(540^{\circ} \mathrm{C}\right)$, and no forward motion. 


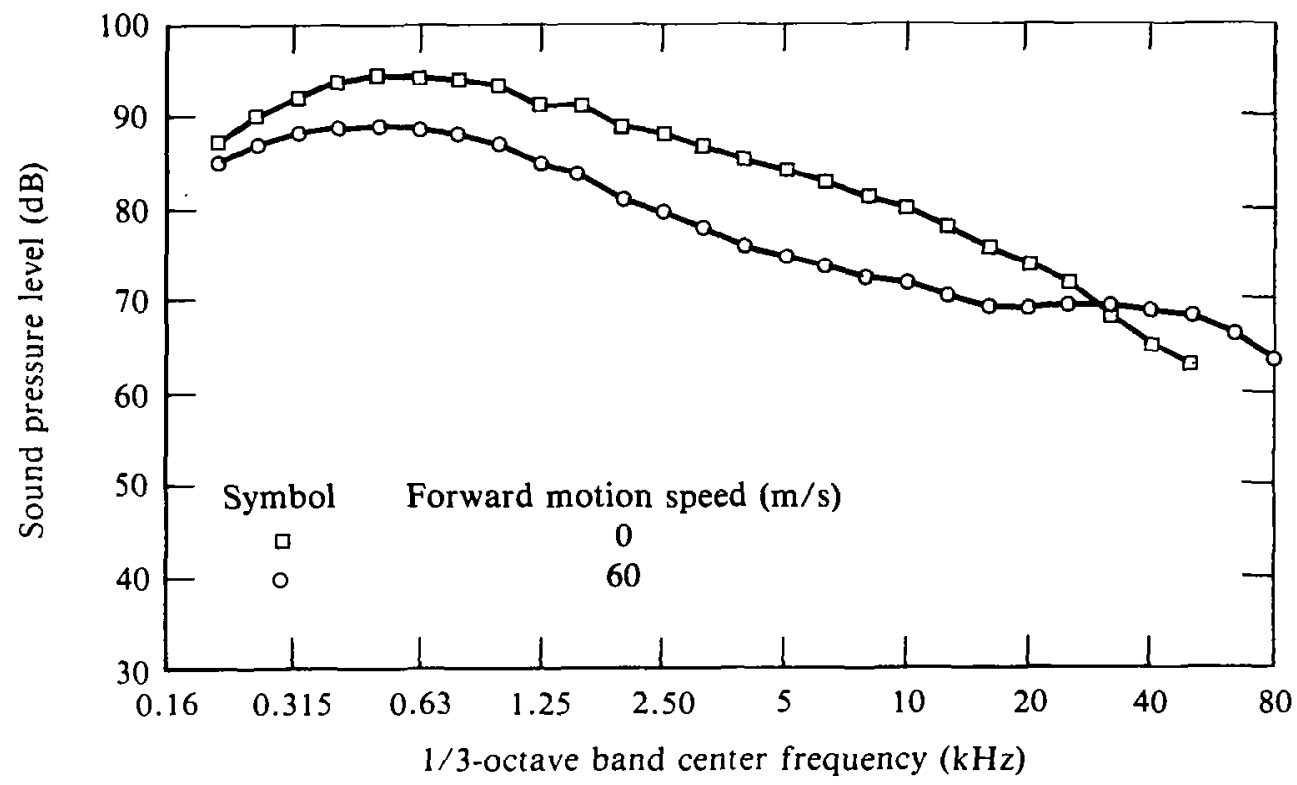

Figure A53. Sound pressure levels as a function of $1 / 3$-octave band frequency; microphone 3 , pressure ratio $=1.6$, cold flow, configuration 1.

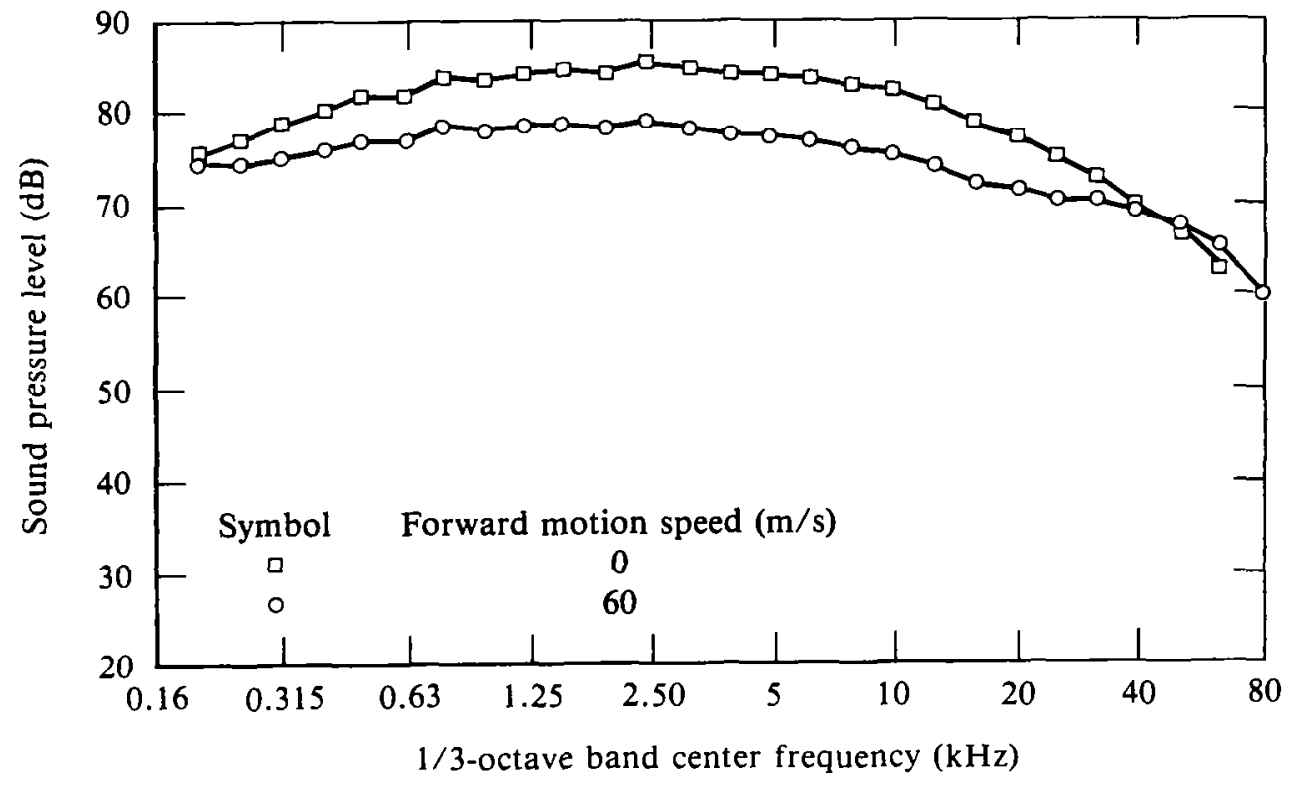

Figure A54. Sound pressure levels as a function of 1/3-octave band frequency; microphone 9 , pressure ratio $=1.6$, cold flow, configuration 1. 


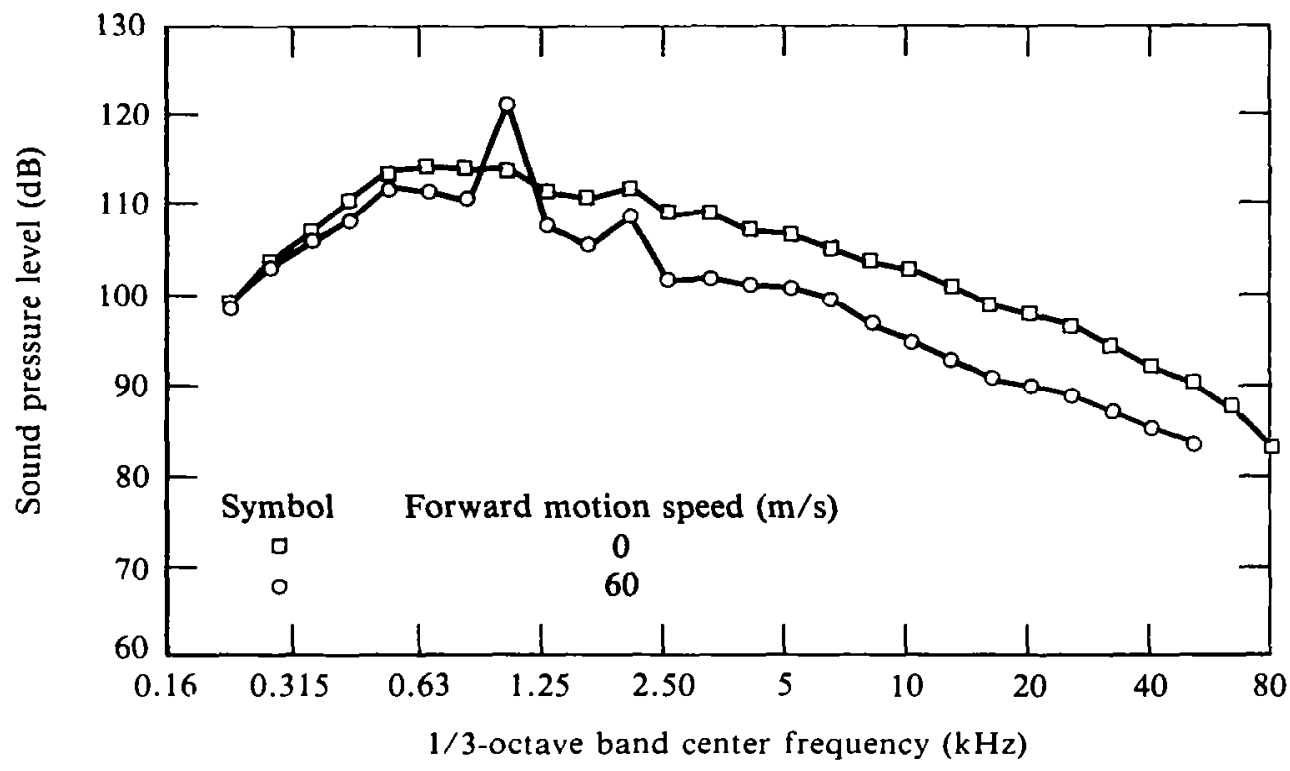

Figure A55. Sound pressure levels as a function of 1/3-octave band frequency; microphone 3 , pressure ratio $=2.8$, cold flow, configuration 1.

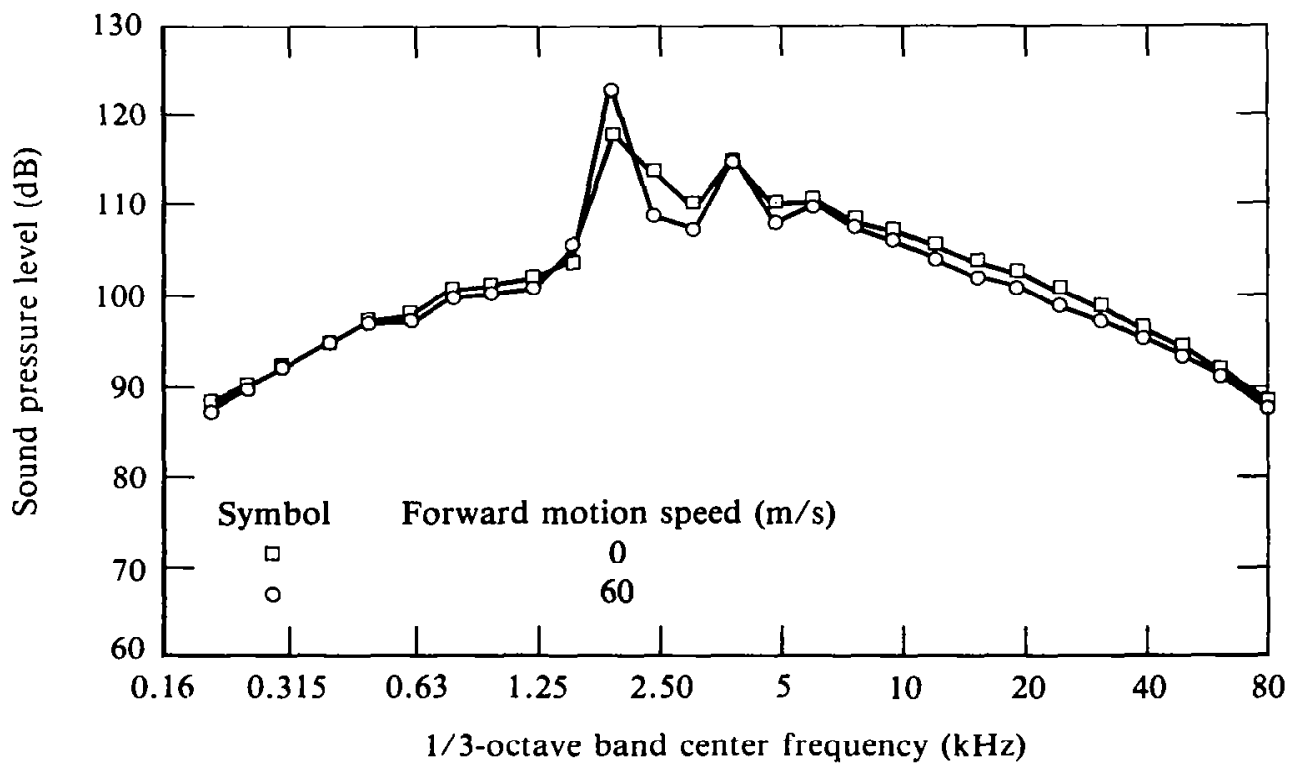

Figure A56. Sound pressure levels as a function of 1/3-octave band frequency; microphone 9 , pressure ratio $=2.8$, cold flow, configuration 1. 


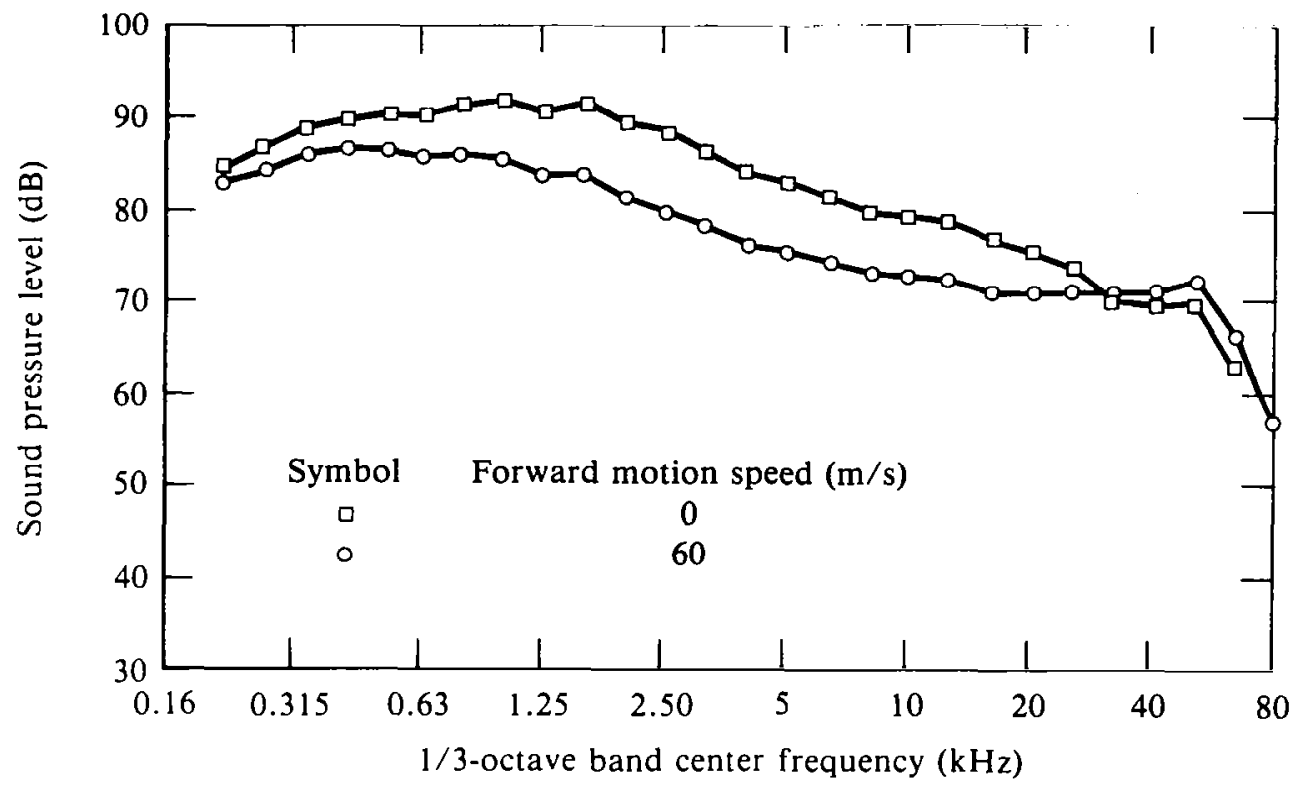

Figure A57. Sound pressure levels as a function of 1/3-octave band frequency; microphone 3 , pressure ratio $=1.6$, cold flow, configuration 4.

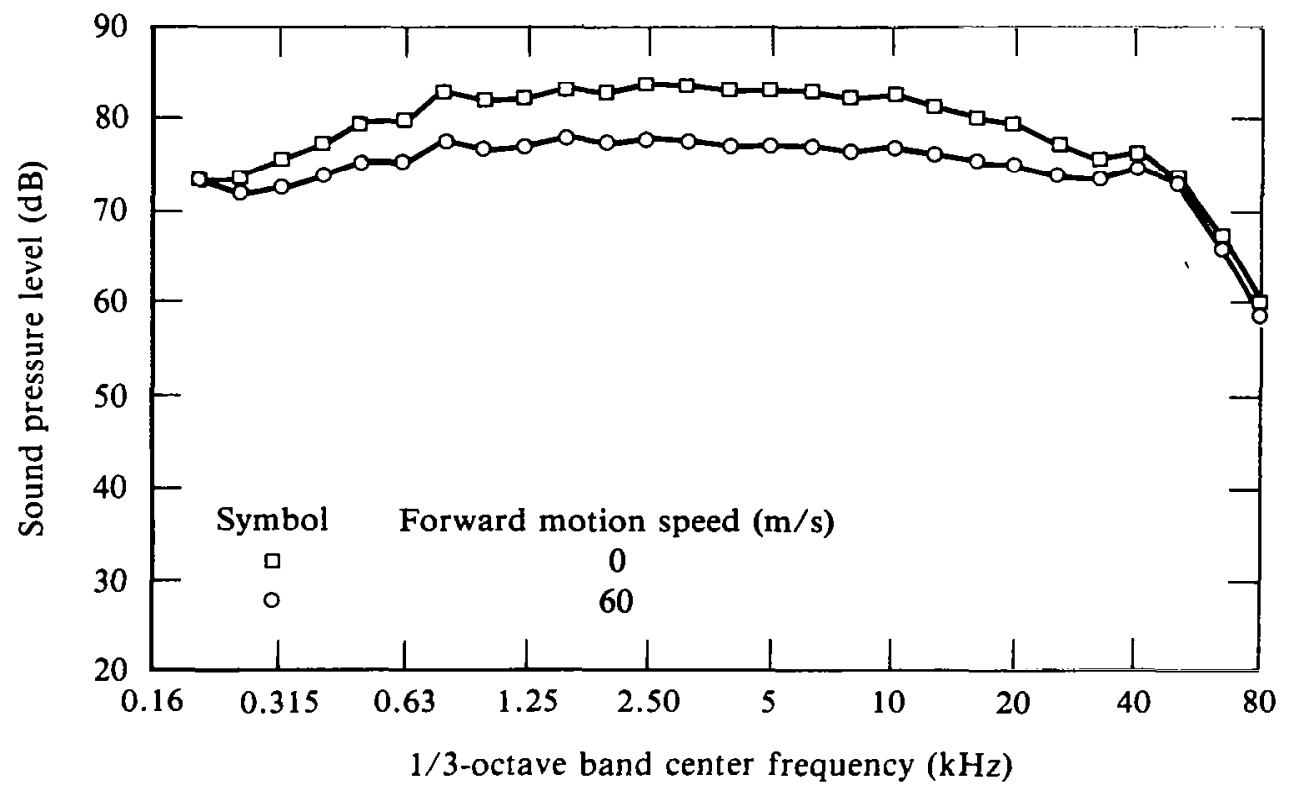

Figure A58. Sound pressure levels as a function of 1/3-octave band frequency; microphone 9 , pressure ratio $=1.6$, cold flow, configuration 4. 


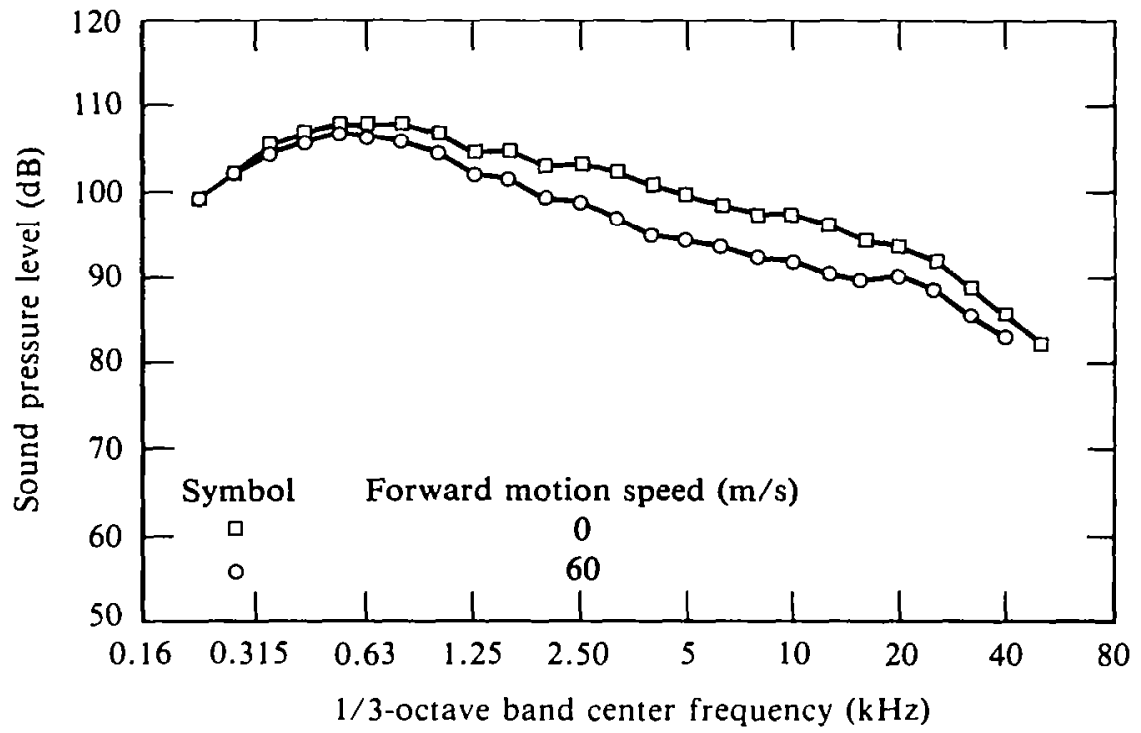

Figure A59. Sound pressure levels as a function of $1 / 3-0 c t a v e$ band frequency; microphone 3 , pressure ratio $=2.8$, cold flow, configuration 4.

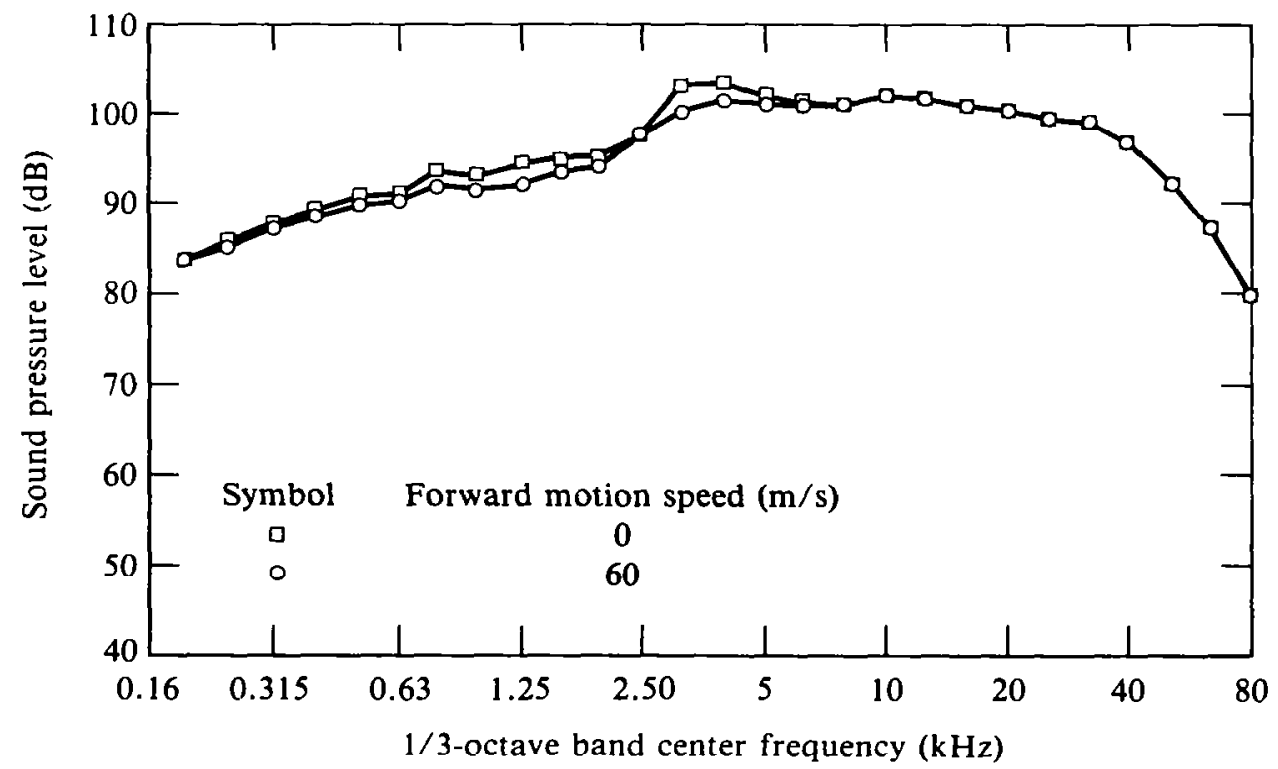

Figure A60. Sound pressure levels as a function of 1/3-octave band frequency; microphone 9 , pressure ratio $=2.8$, cold flow, configuration 4. 


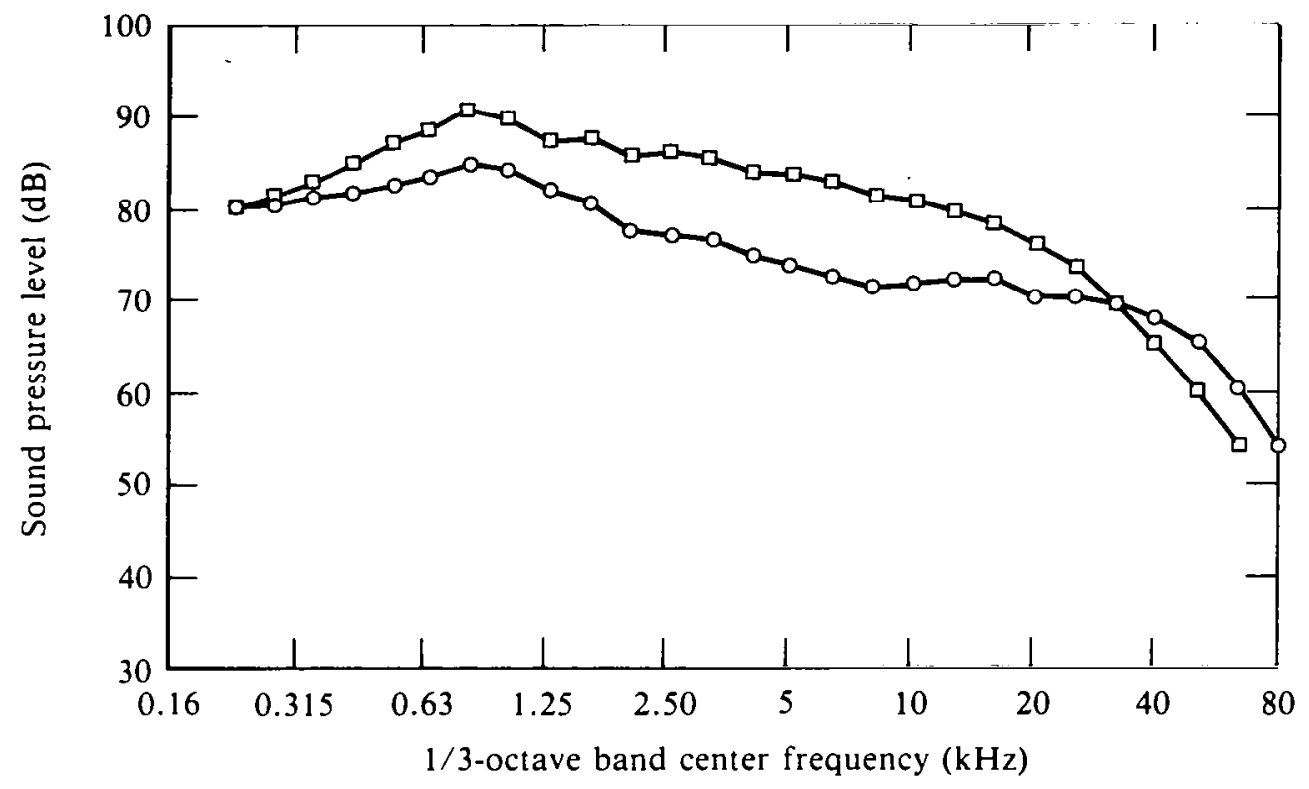

Figure A61. Sound pressure levels as a function of 1/3-octave band frequency; microphone 3 , pressure ratio $=1.6$, cold flow, and configuration 9.

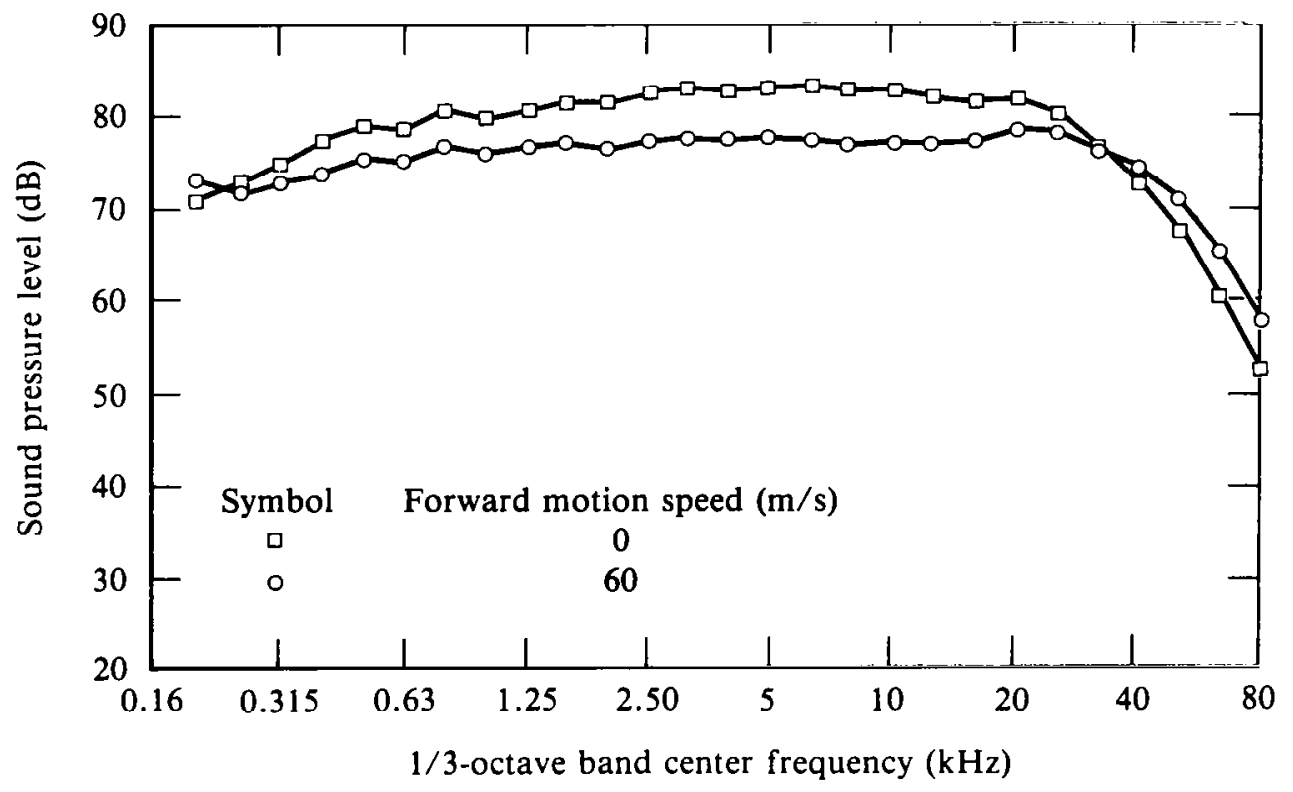

Figure A62. Sound pressure levels as a function of 1/3-octave band frequency; microphone 9 , pressure ratio $=1.6$, cold flow, configuration 9. 


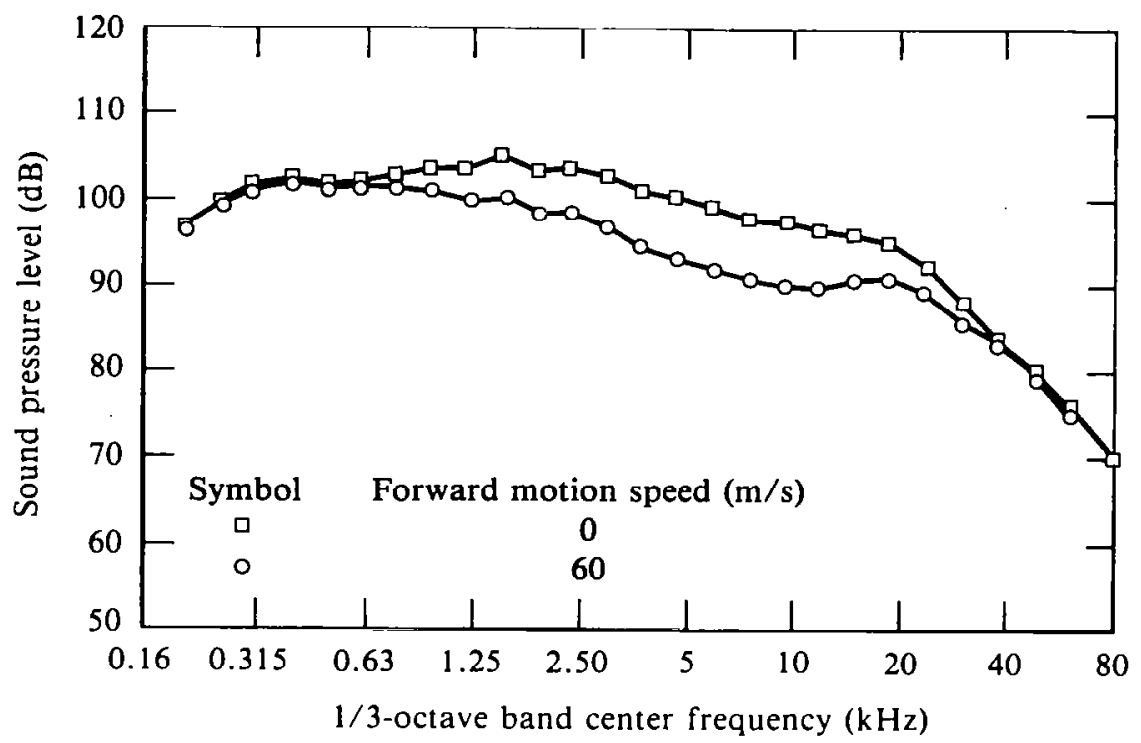

Figure A63. Sound pressure levels as a function of 1/3-octave band frequency; microphone 3 , pressure ratio $=2.8$, cold flow, configuration 9.

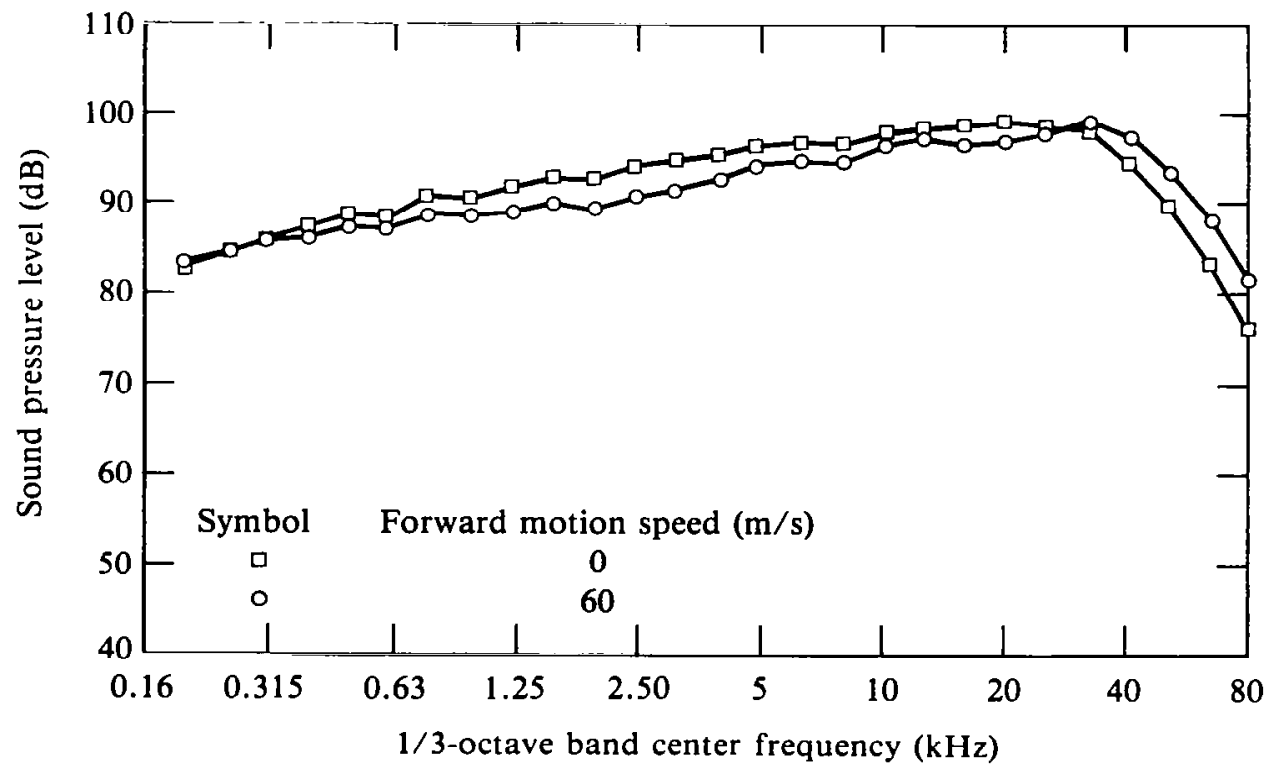

Figure A64. Sound pressure levels as a function of 1/3-octave band frequency; microphone 9 , pressure ratio $=2.8$, cold flow, configuration 9. 


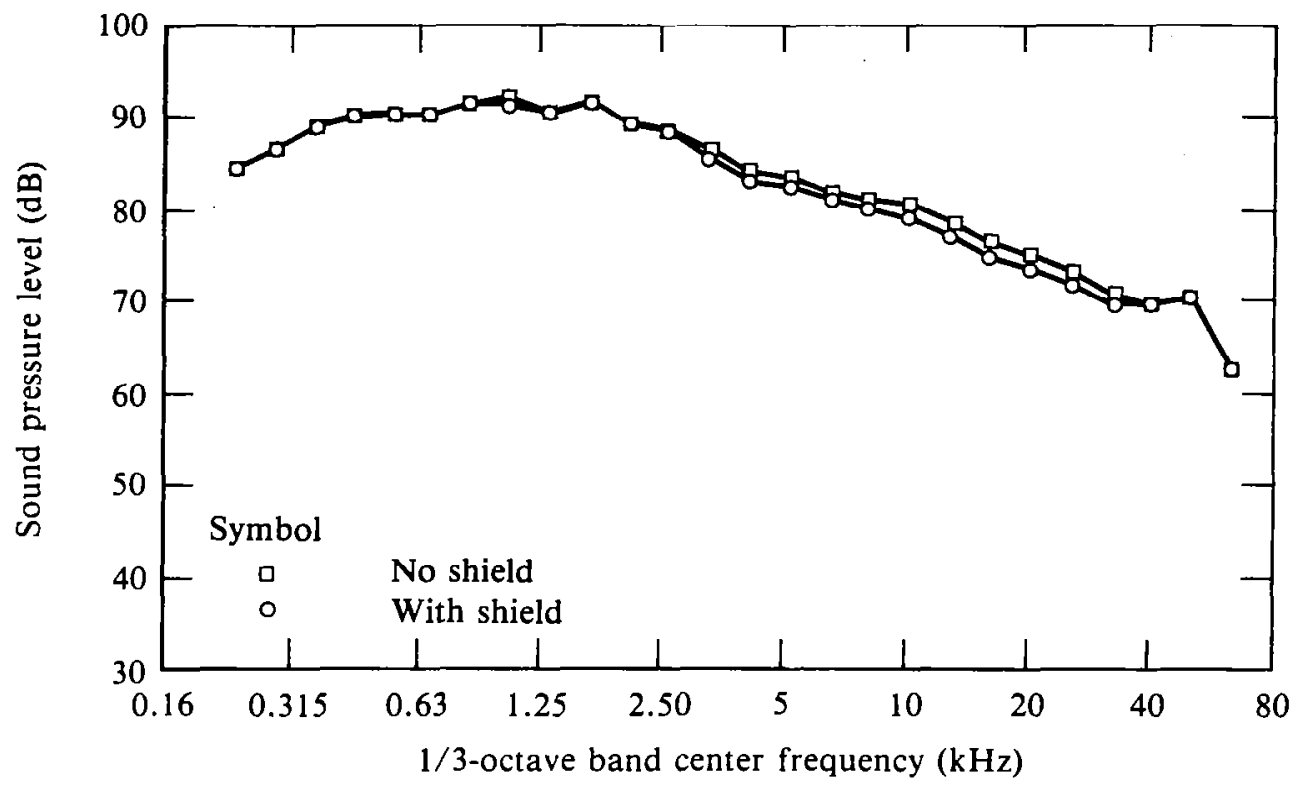

Figure A65. Sound pressure levels as a function of 1/3-octave band frequency; microphone 3 , pressure ratio $=1.6$, cold flow, no forward motion, configuration 4.

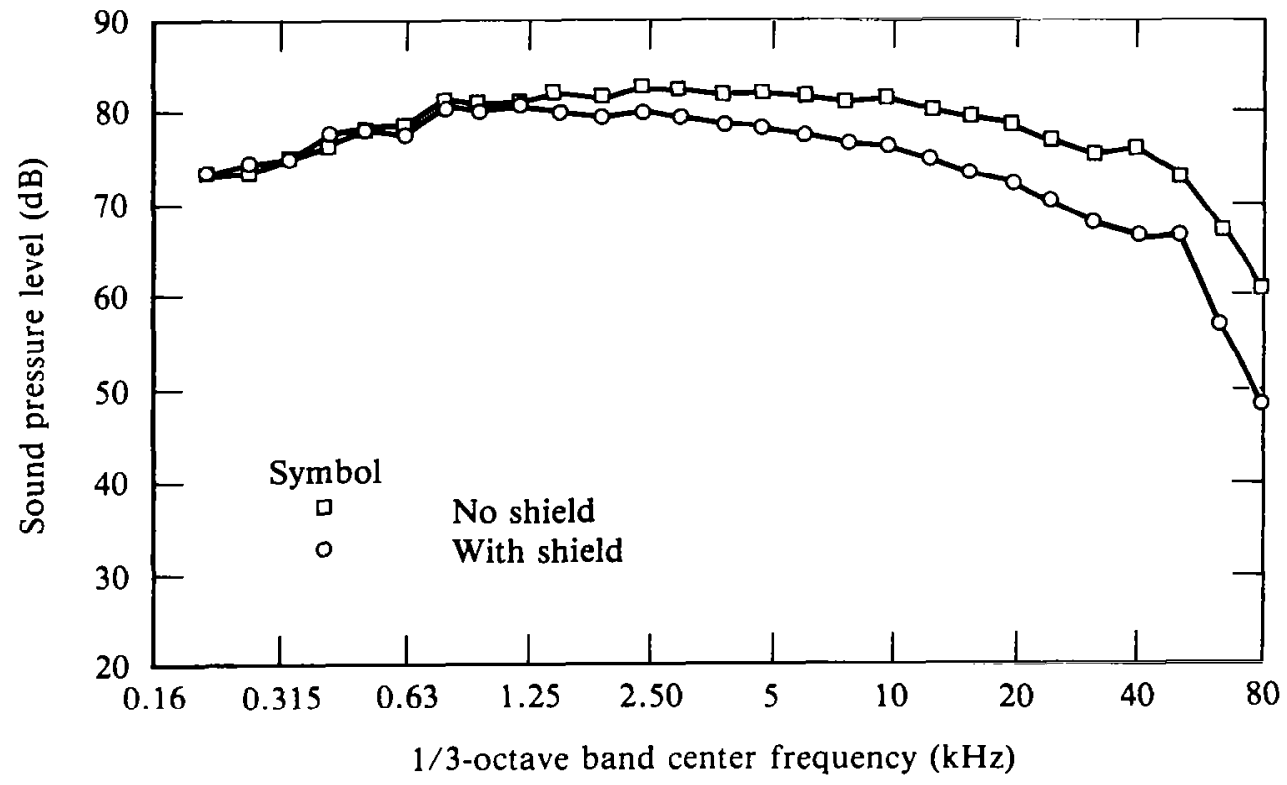

Figure A66. Sound pressure levels as a function of 1/3-octave band frequency; microphone 9 , pressure ratio $=1.6$, cold flow, no forward motion, configuration 4. 


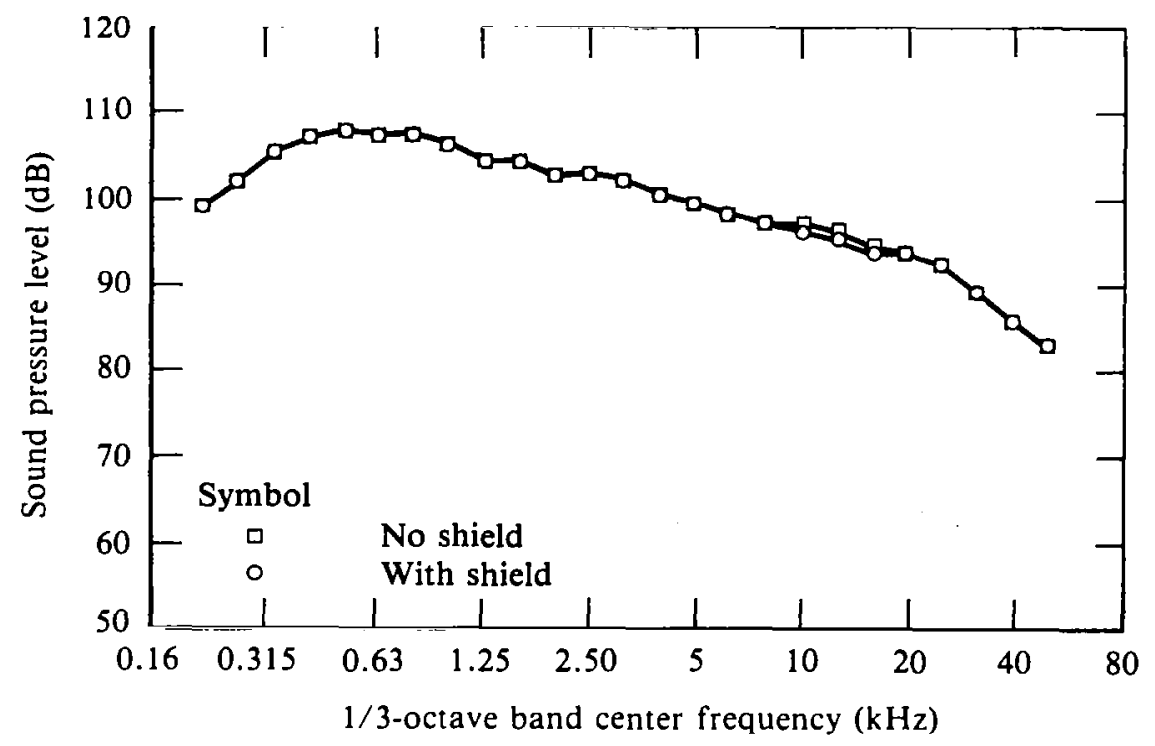

Figure A67. Sound pressure levels as a function of 1/3-octave band frequency; microphone 3 , pressure ratio $=2.8$, cold flow, no forward motion, configuration 4.

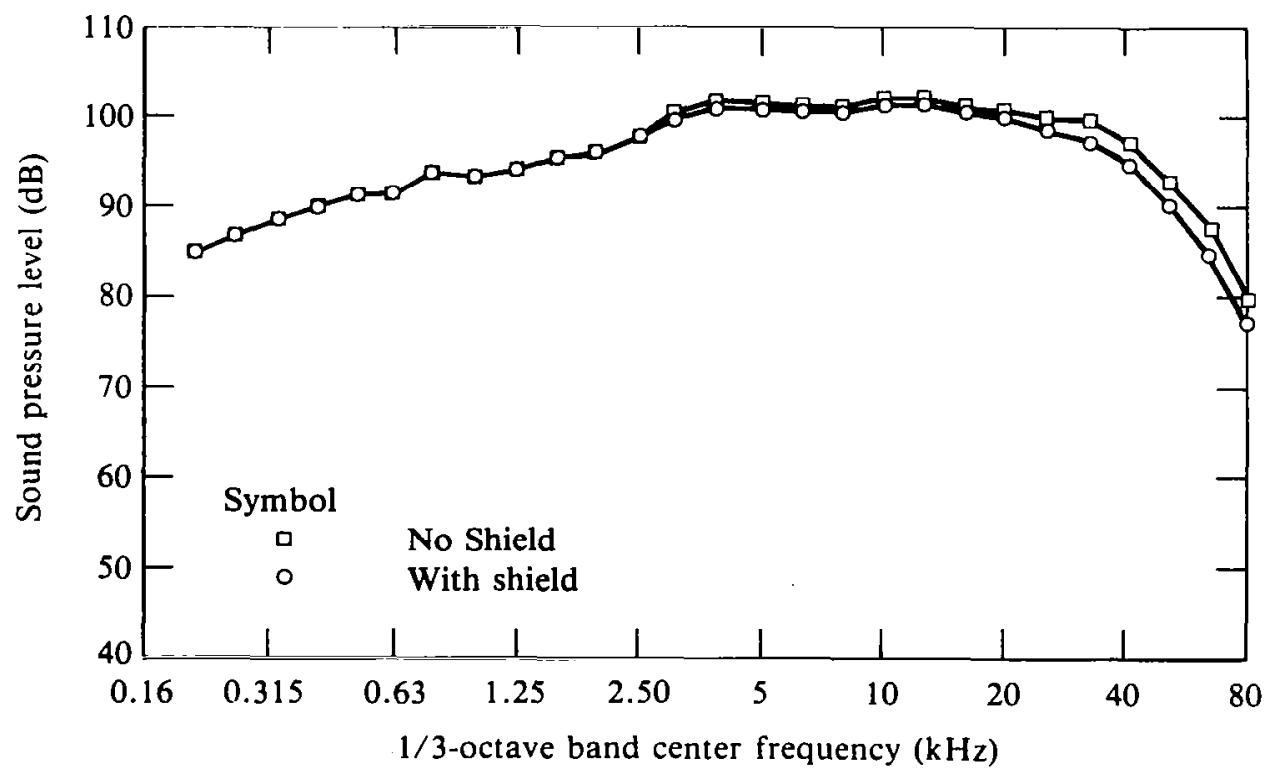

Figure A68. Sound pressure levels as a function of 1/3-octave band frequency; microphone 9 , pressure ratio $=2.8$, cold flow, no forward motion, configuration 1. 


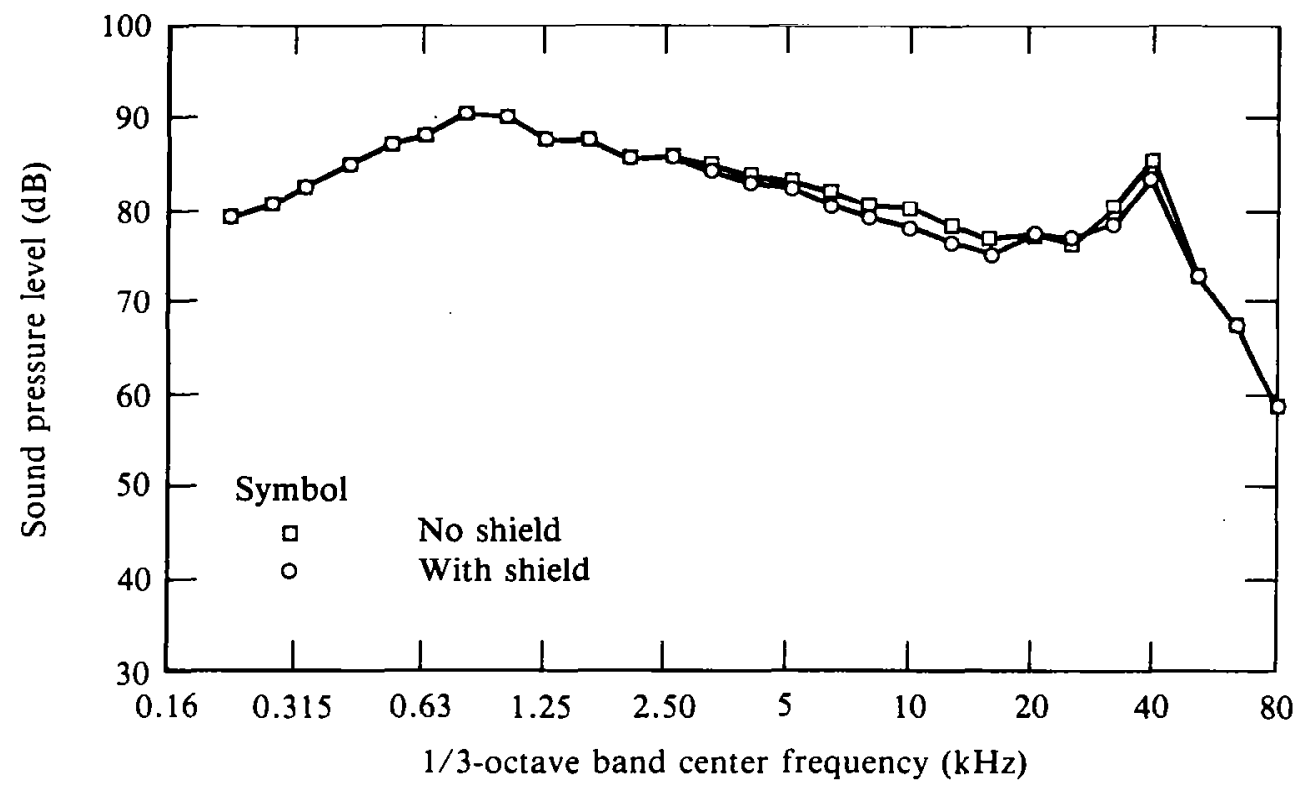

Figure A69. Sound pressure levels as a function of 1/3-octave band frequency; microphone 3 , pressure ratio $=1.6$, cold flow, no forward motion, configuration 10.

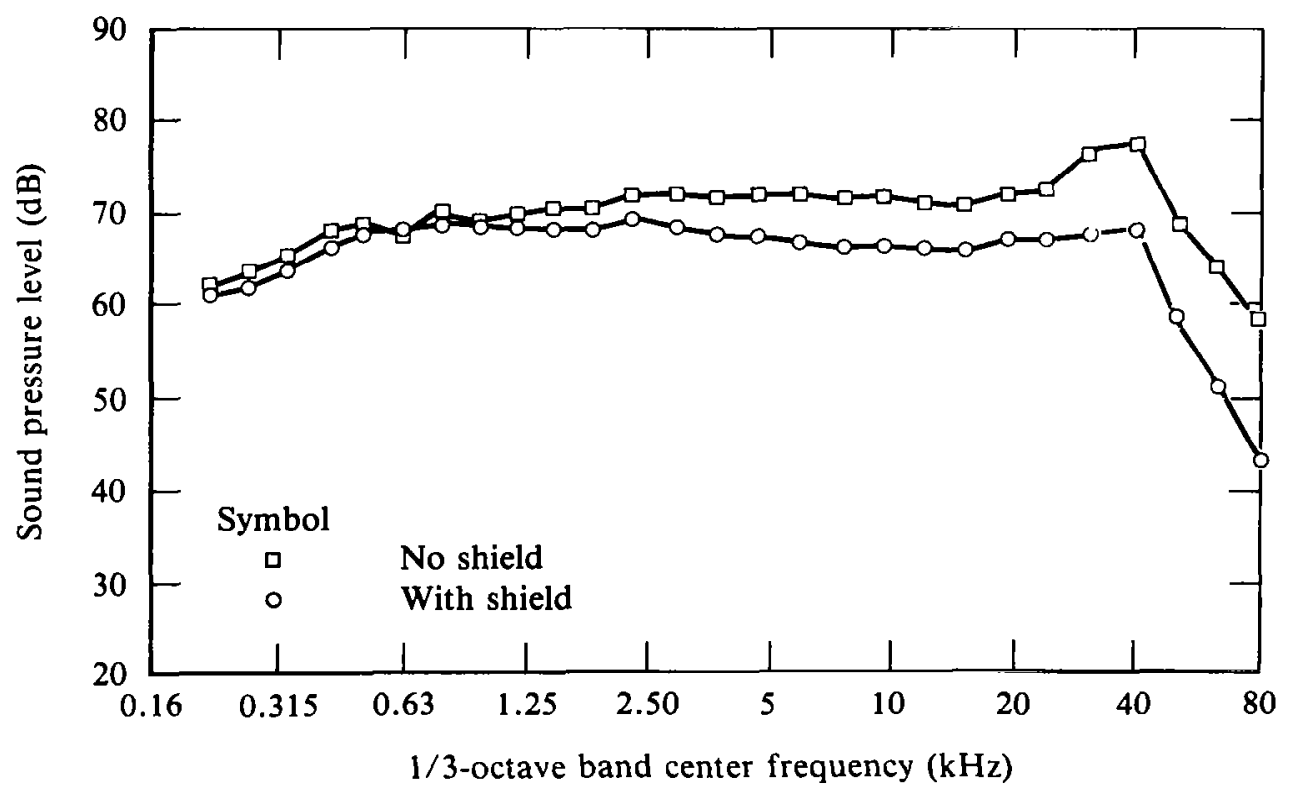

Figure A70. Sound pressure levels as a function of $1 / 3$-octave band frequency; microphone 9 , pressure ratio $=1.6$, cold flow, no forward motion, configuration 10. 


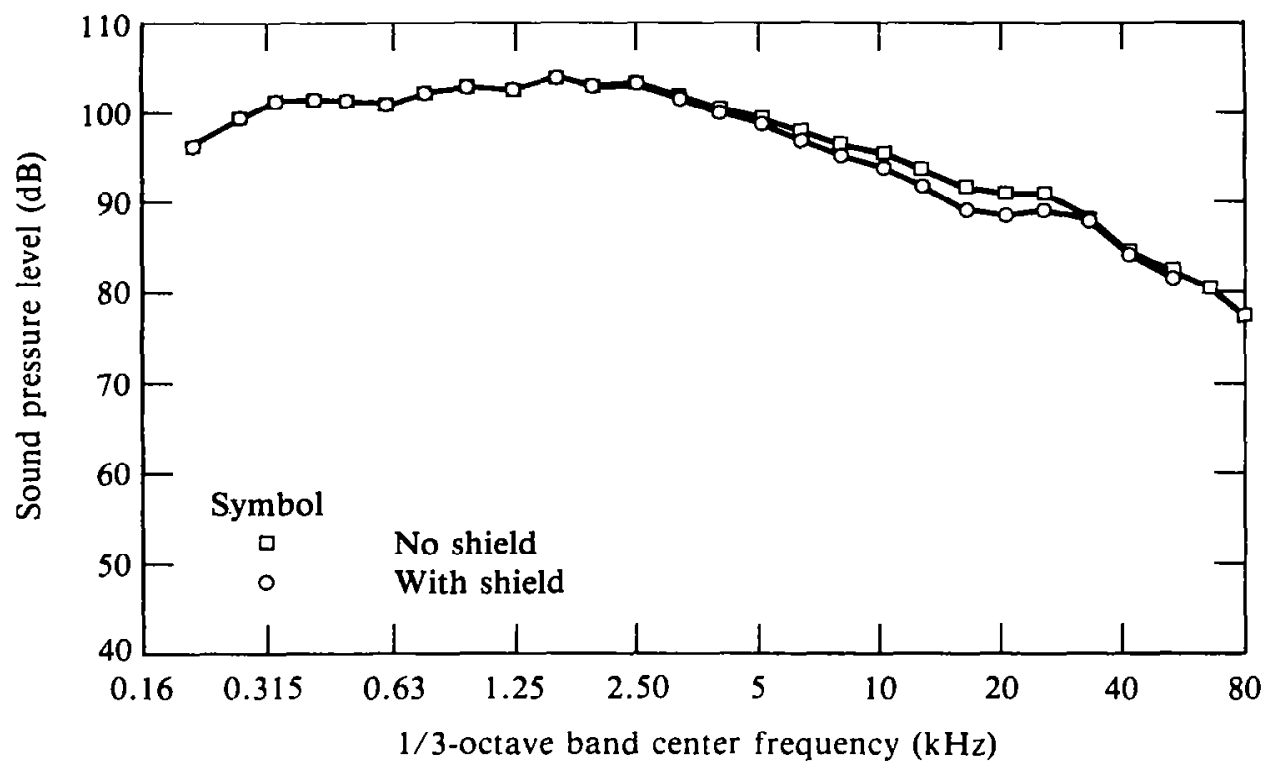

Figure A71. Sound pressure levels as a function of 1/3-octave band frequency; microphone 3 , pressure ratio $=2.8$, cold flow, no forward motion, configuration 10.

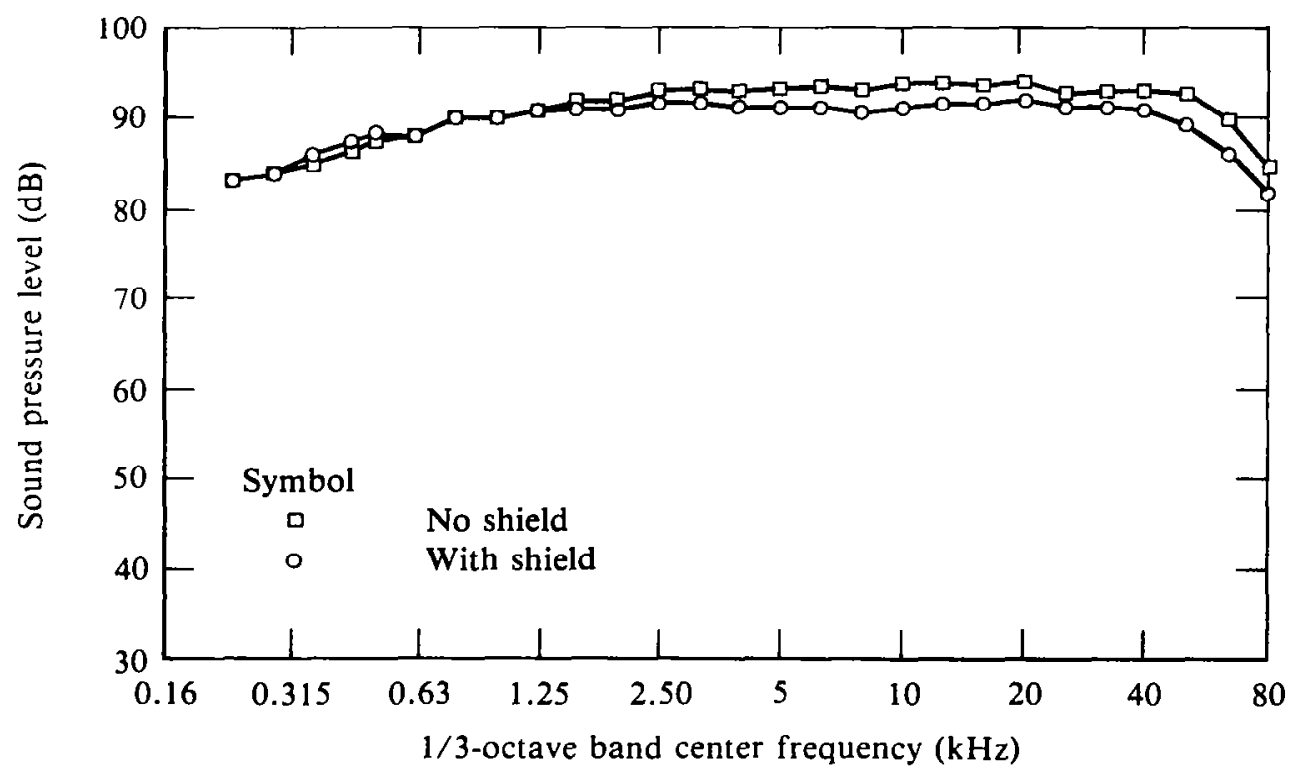

Figure A72. Sound pressure levels as a function of 1/3-octave band frequency; microphone 9 , pressure ratio $=2.8$, cold flow, no forward motion, configuration 10. 
APPENDIX B

SUPPLEMENTARY AERODYNAMIC TEST RESULTS 
NPR 4.0

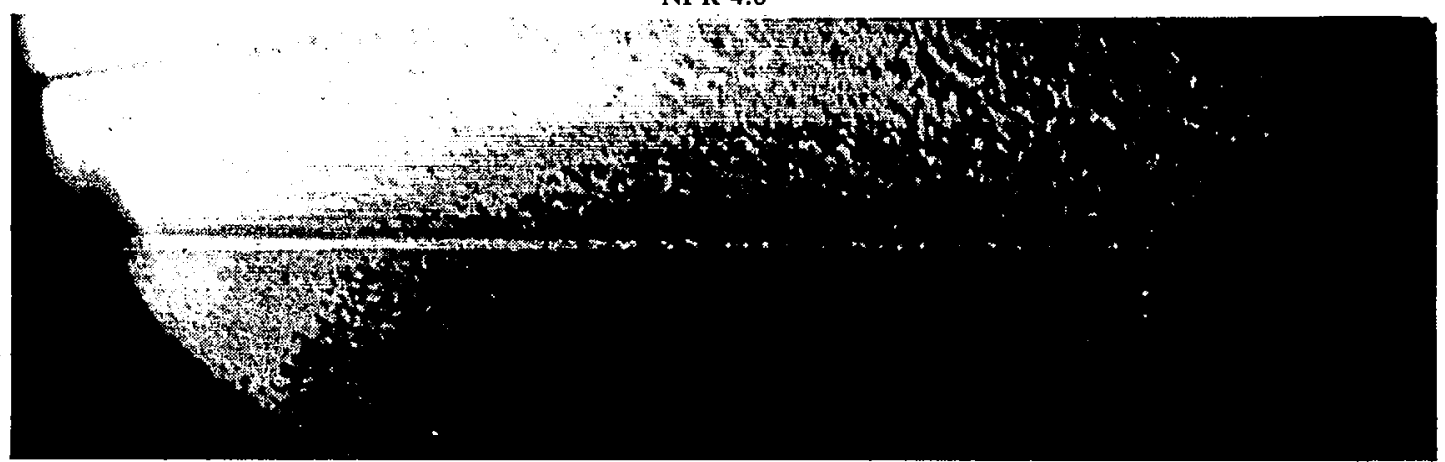

NPR 3.4

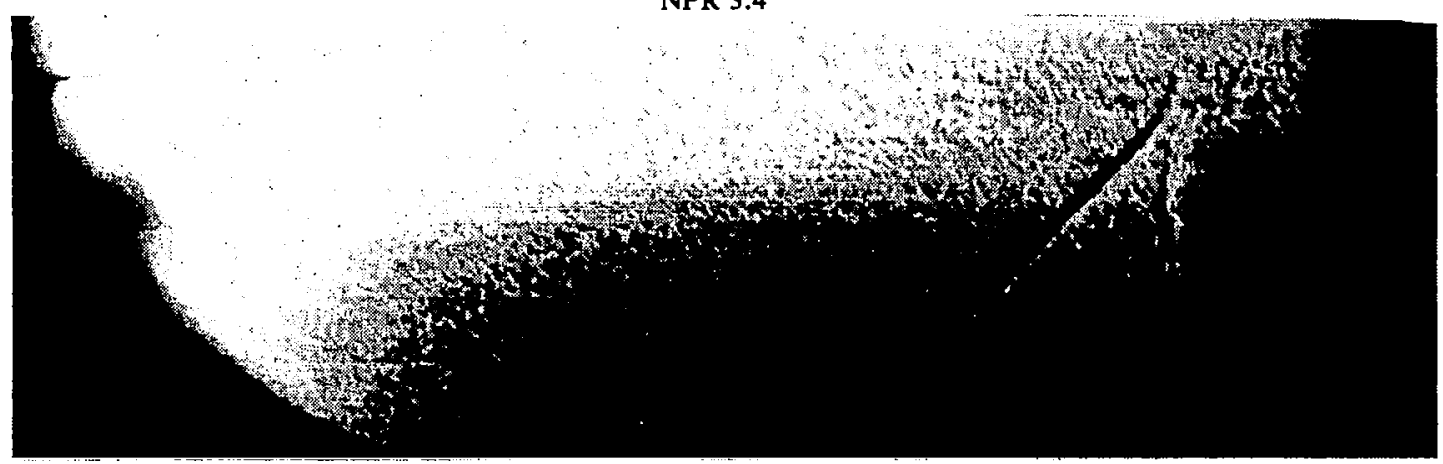

NPR 2.8

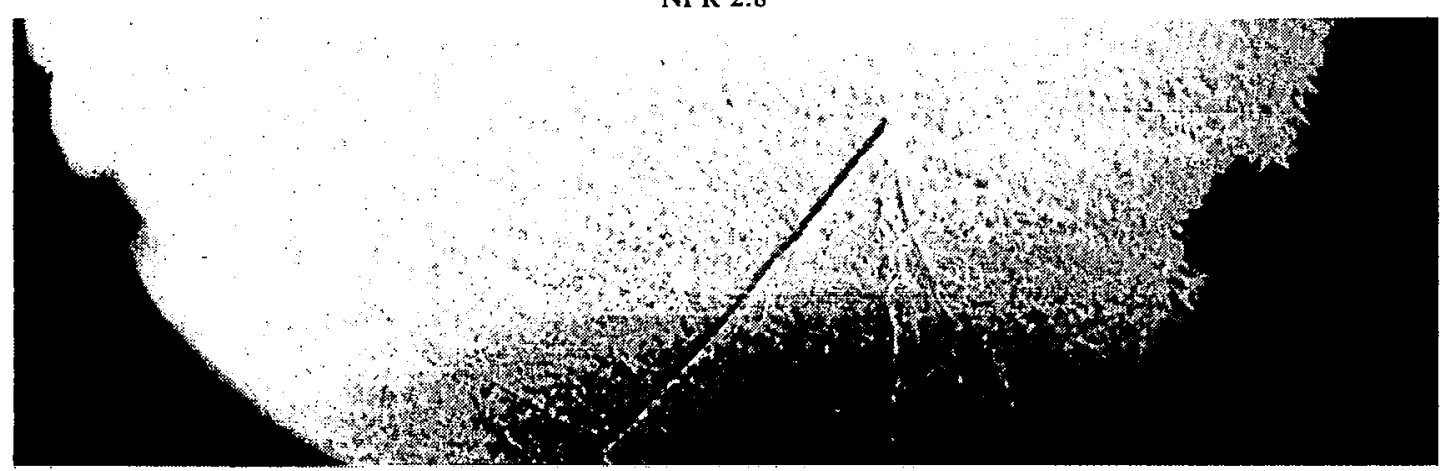

NPR 2.2

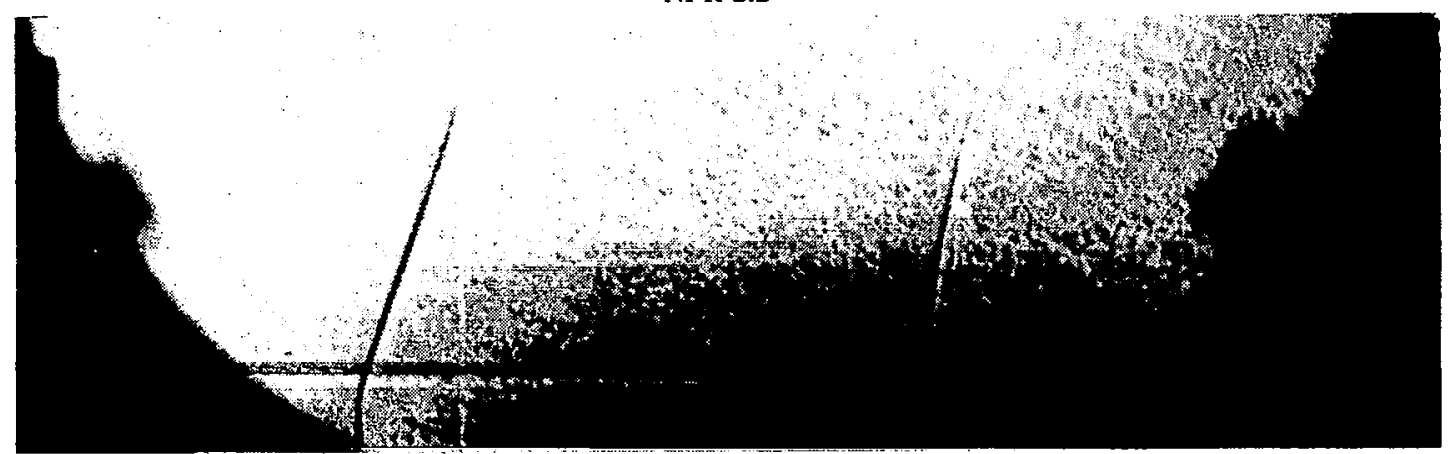

Figure B1. Shadowgraph visualization for Configuration $2(0,0,0)$. 

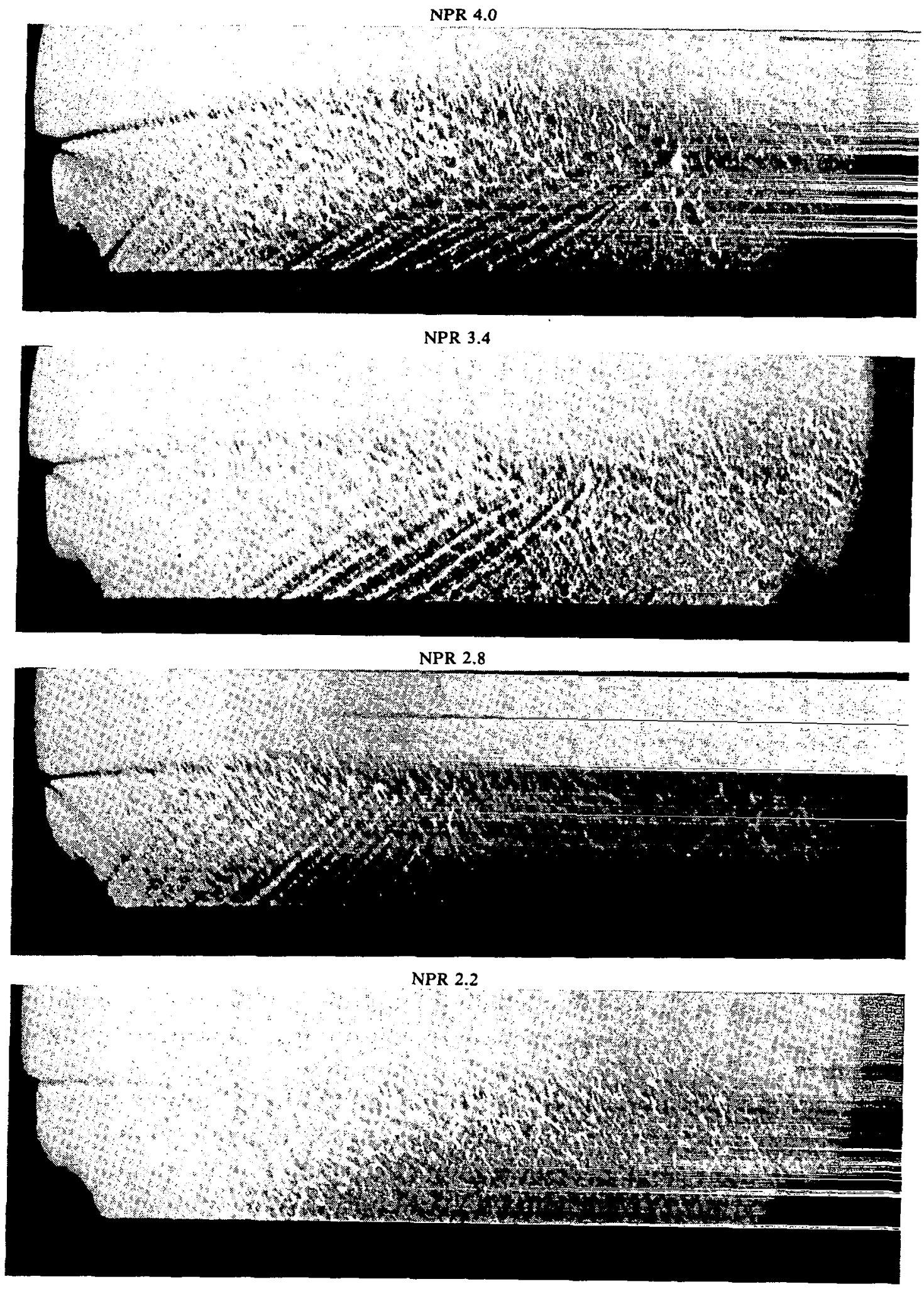

Figure B2. Shadowgraph visualization for Configuration $6(0.03,0.53,3.4)$. 
NPR 4.0

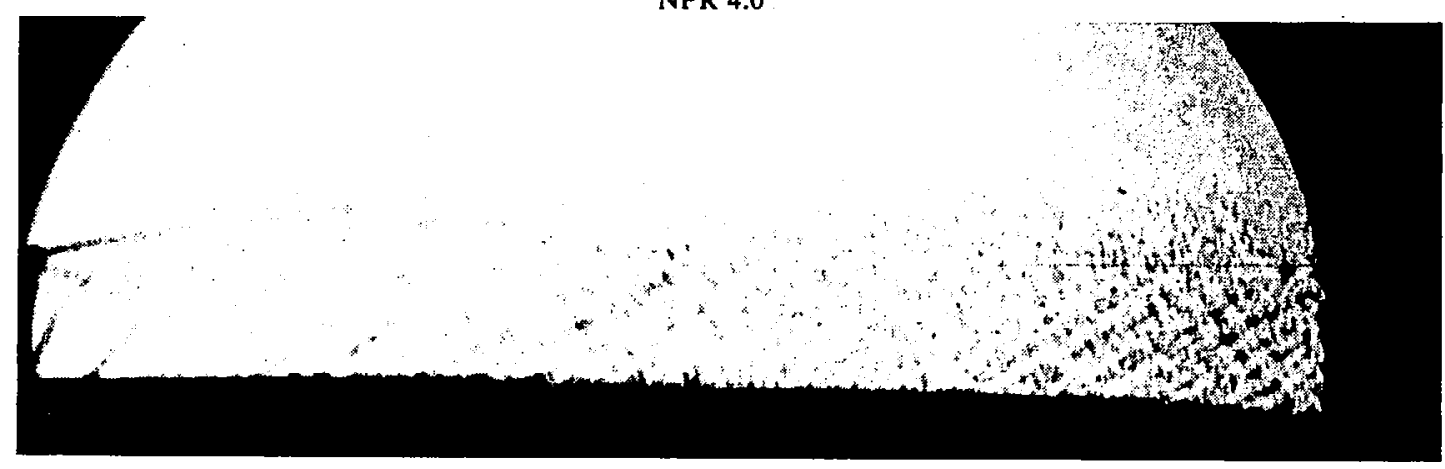

NPR 3.4

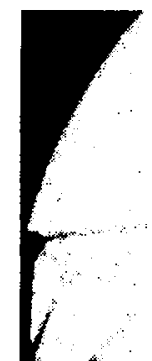

NPR 2.8

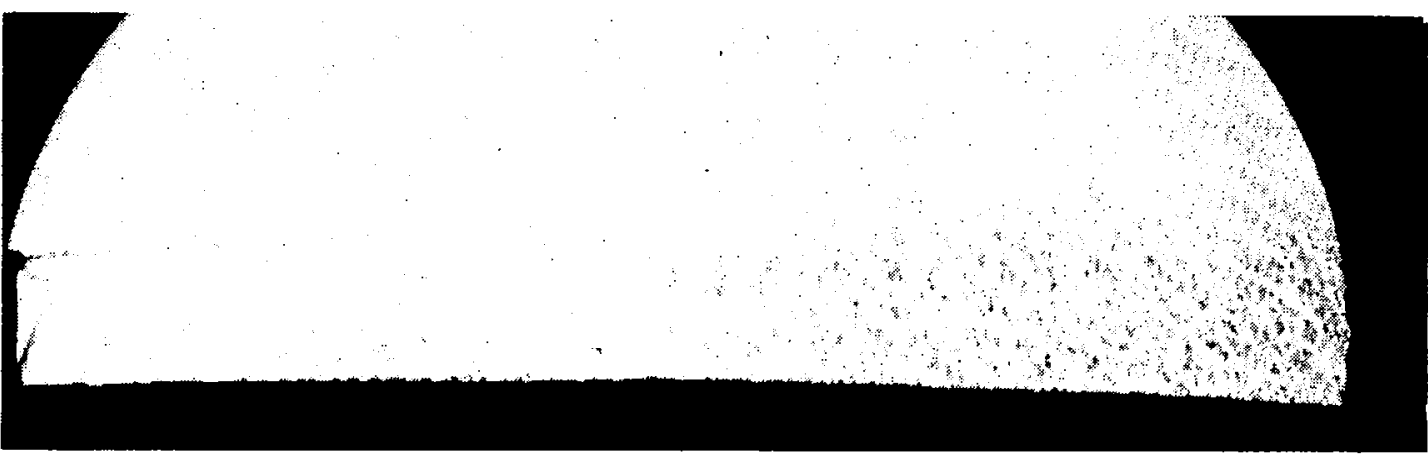

NPR 2.2
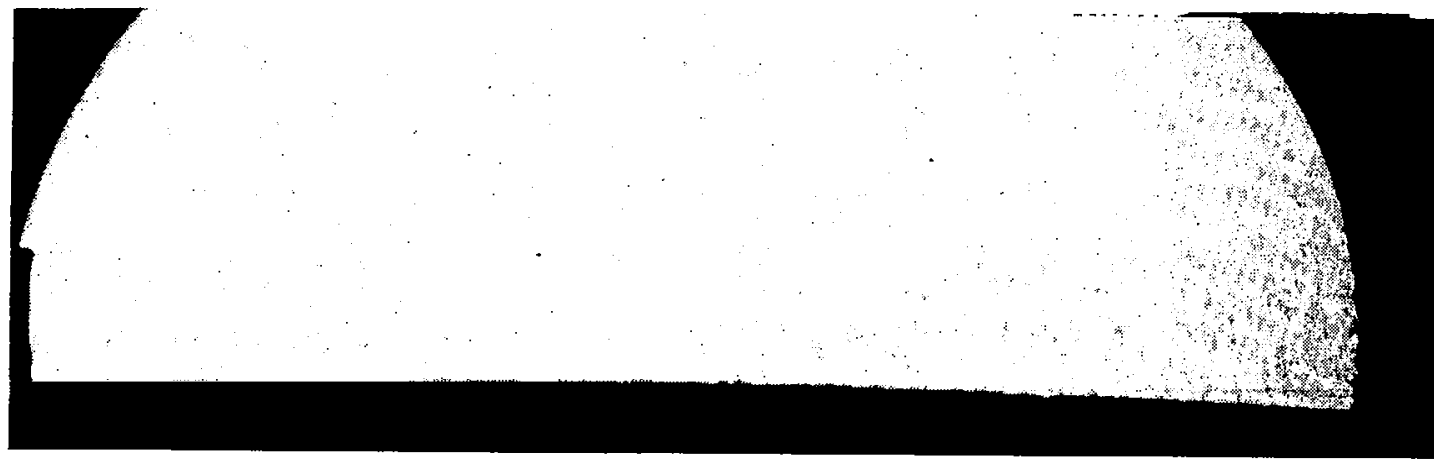

Figure B3. Shadowgraph visualization for Configuration $8(0.03,0.80,3.7)$. 
NPR 4.0

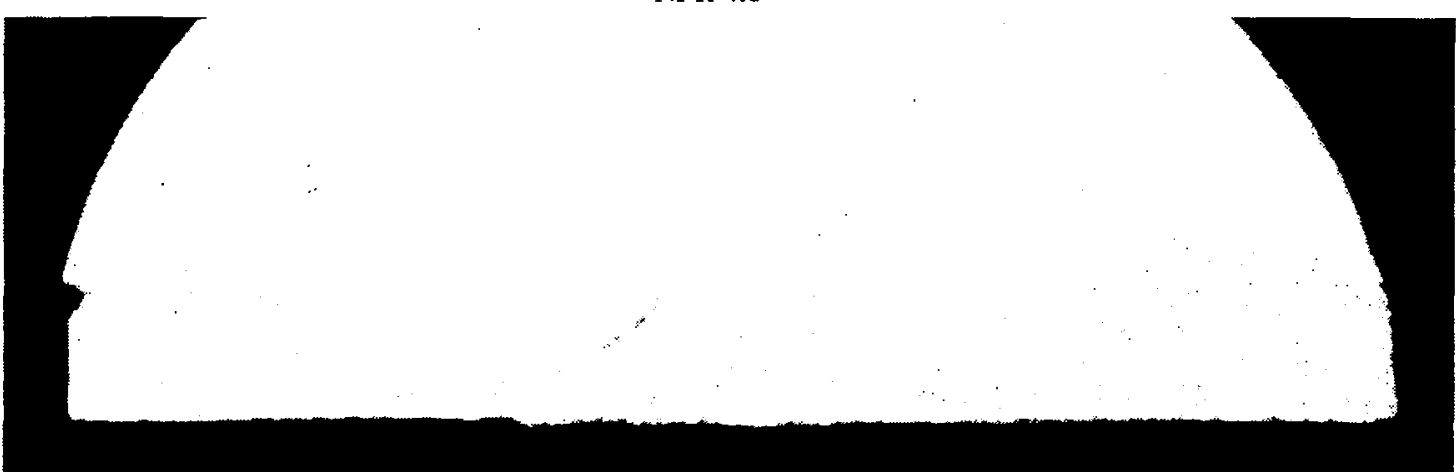

NPR 3.4

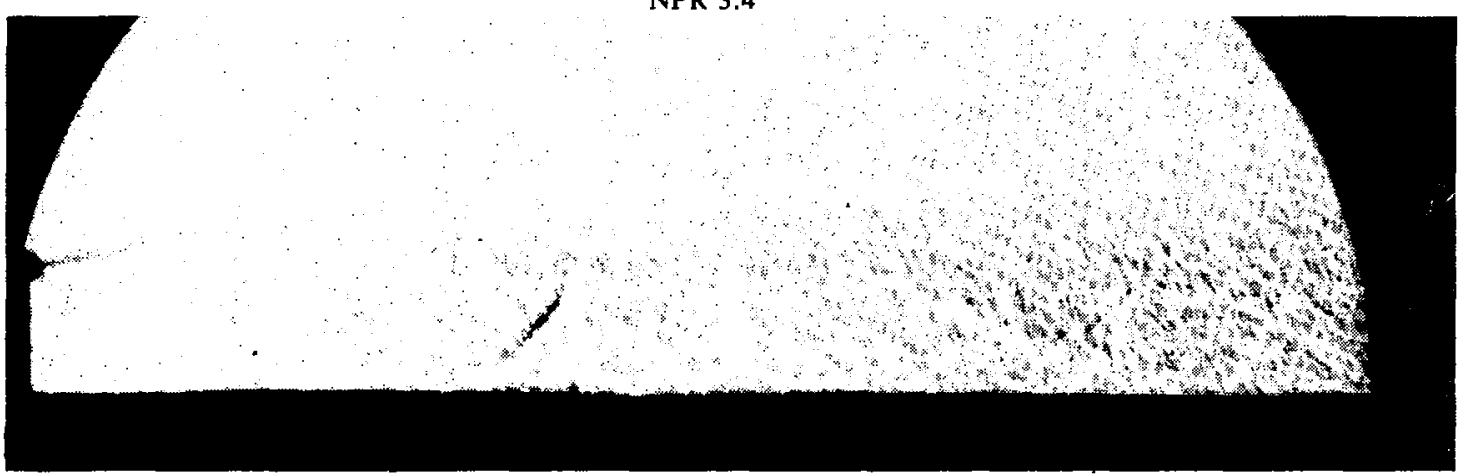

NPR 2.8

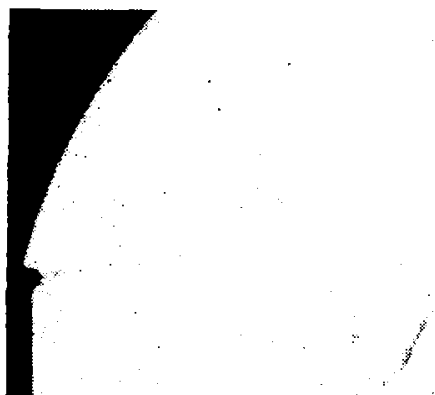

NPR 2.2

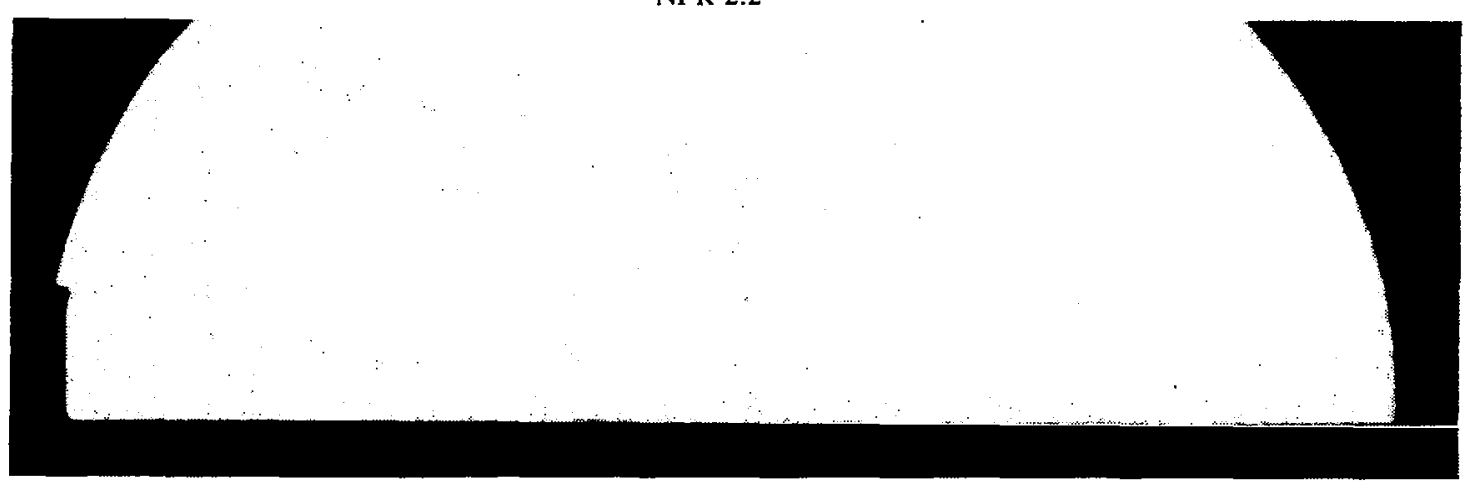

Figure B4. Shadowgraph visualization for Configuration $9 T(0,0.80,5.2)$. 
NPR 4.0

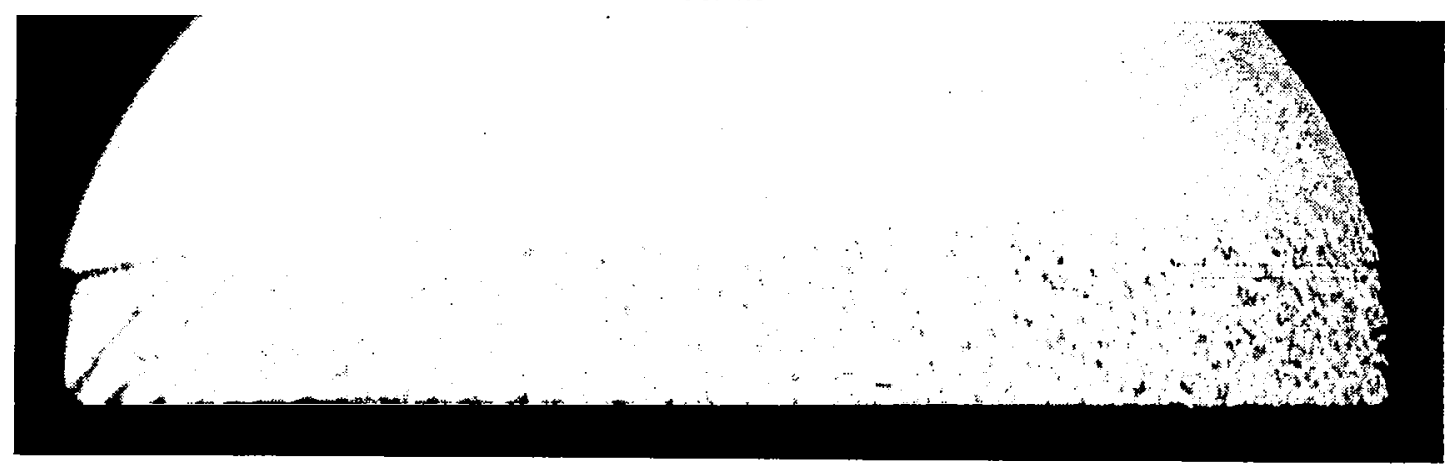

NPR 4.0

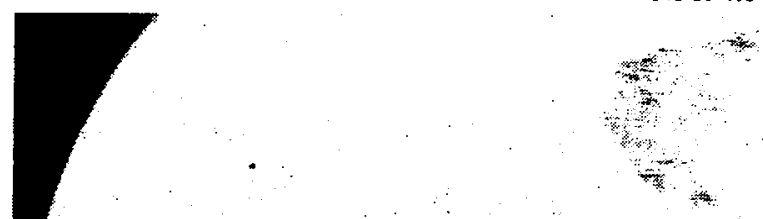

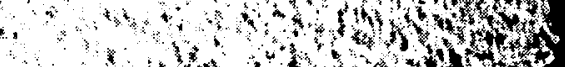

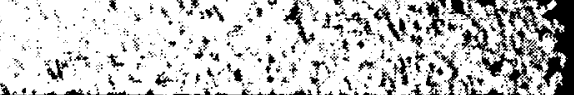

NPR 2.8
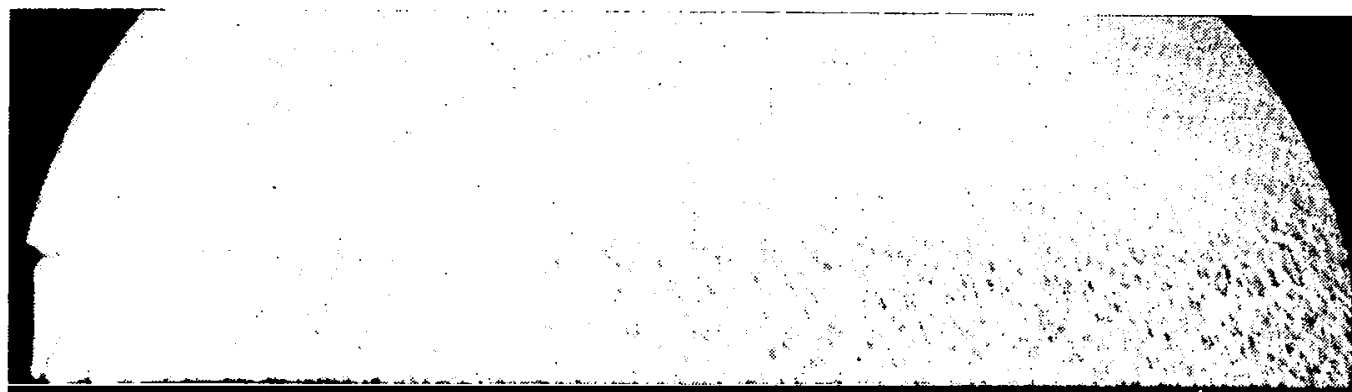

NPR 2.2
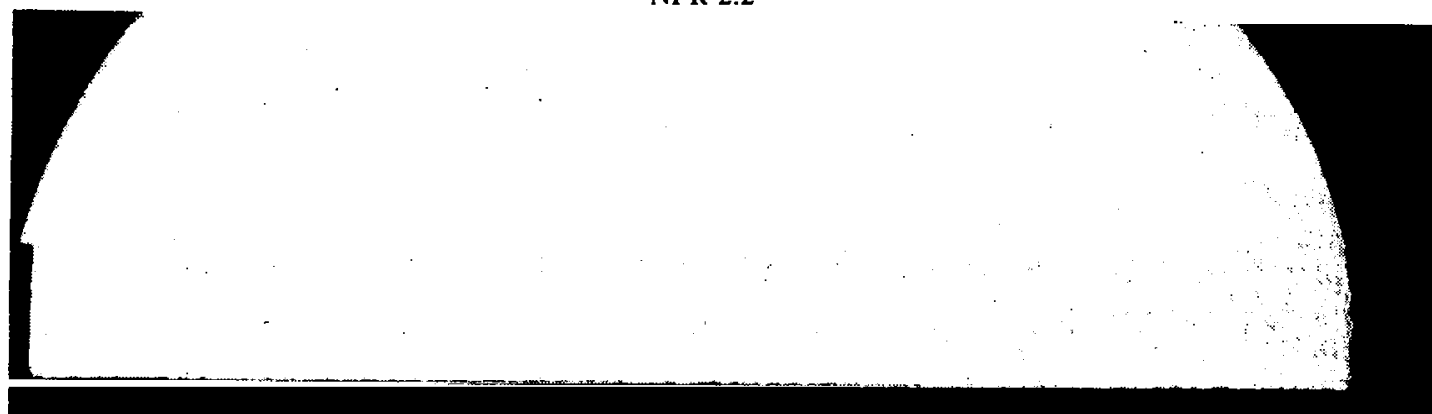

Figure B5. Shadowgraph visualization for Configuration $10(0.06,0.80,5.2)$. 

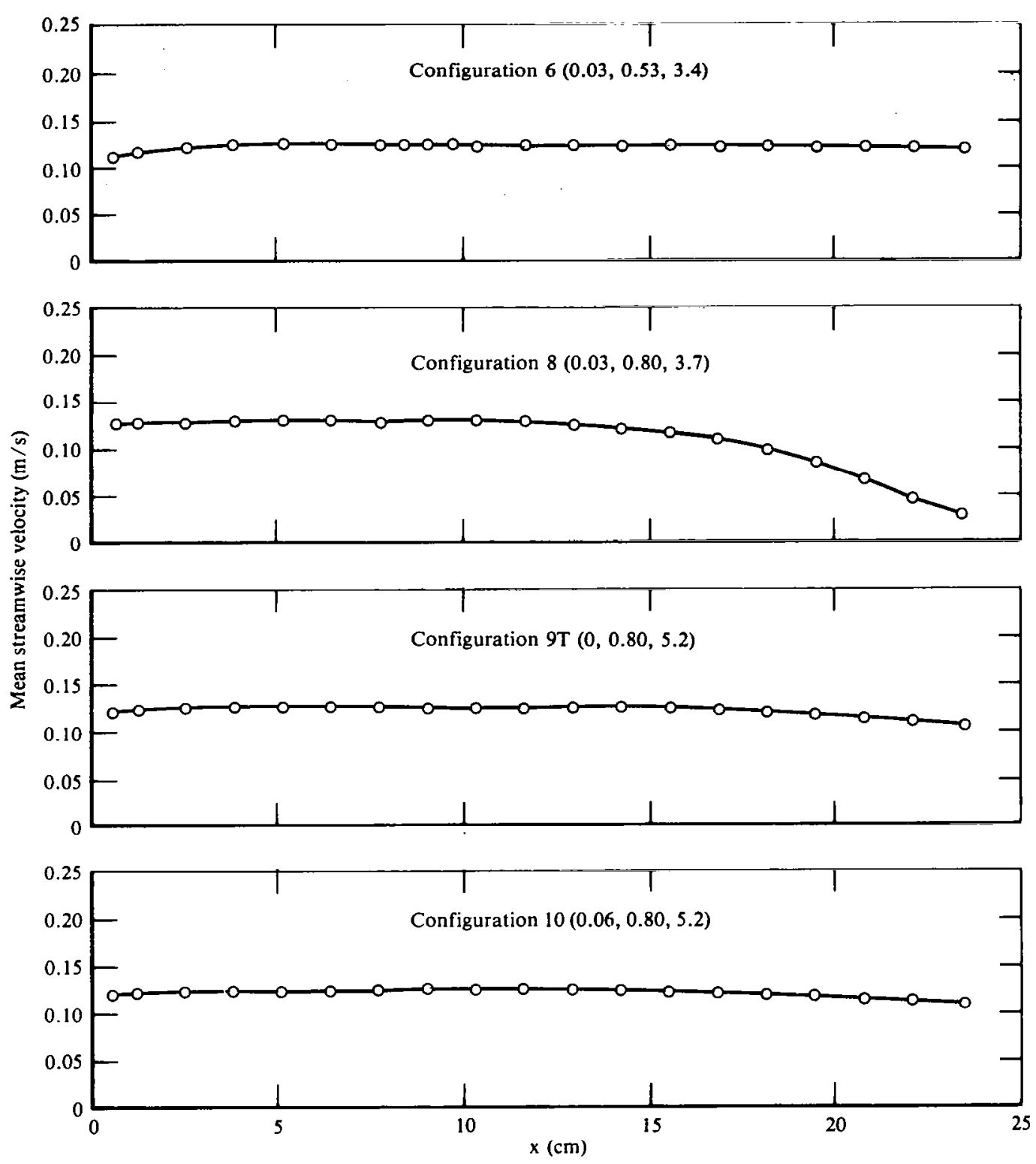

Figure B6. Mean streamwise velocity profiles at a nozzle pressure ratio of 1.6 . 

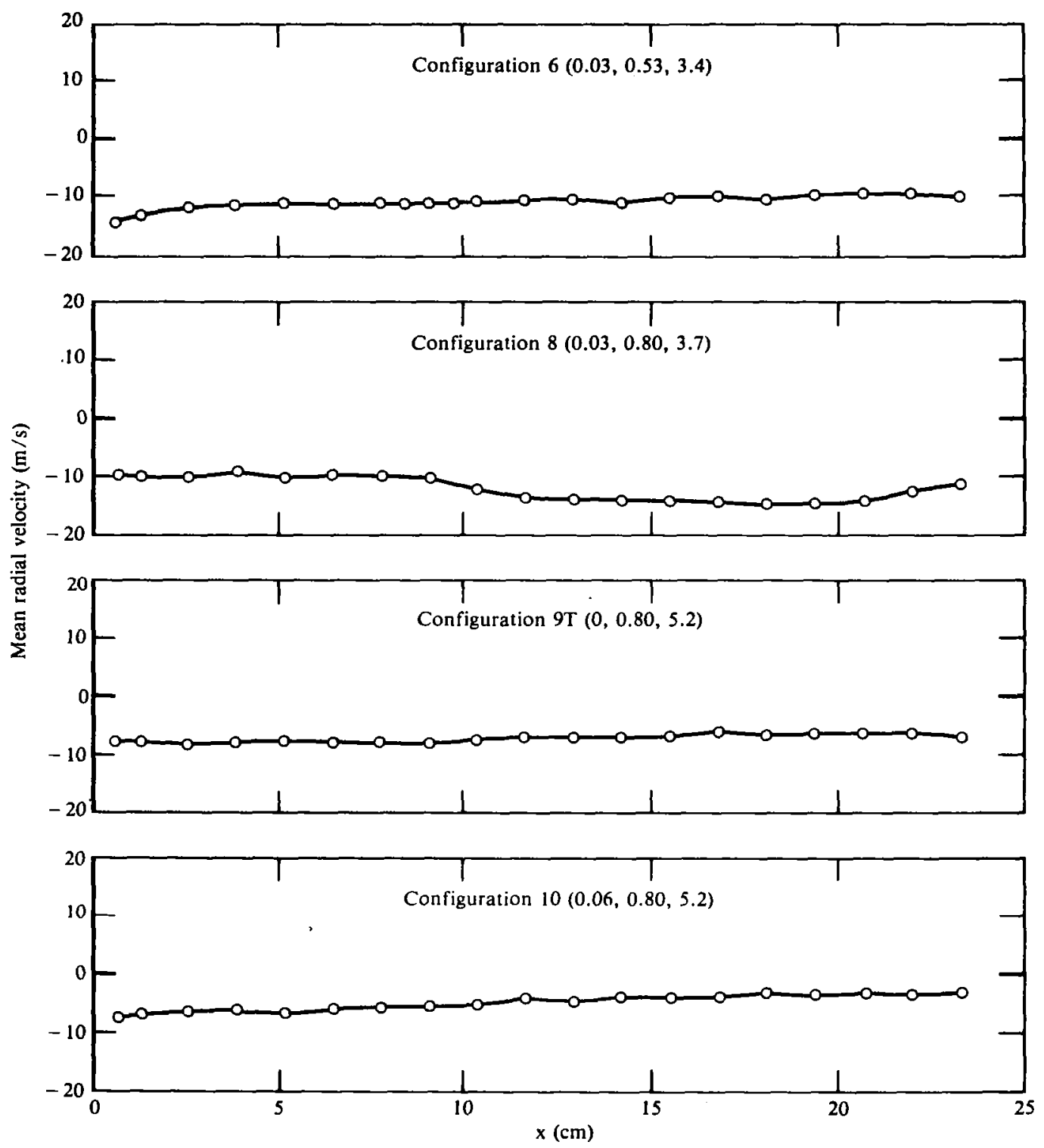

Figure B7. Mean radial velocity profiles at a nozzle pressure ratio of 1.6. 

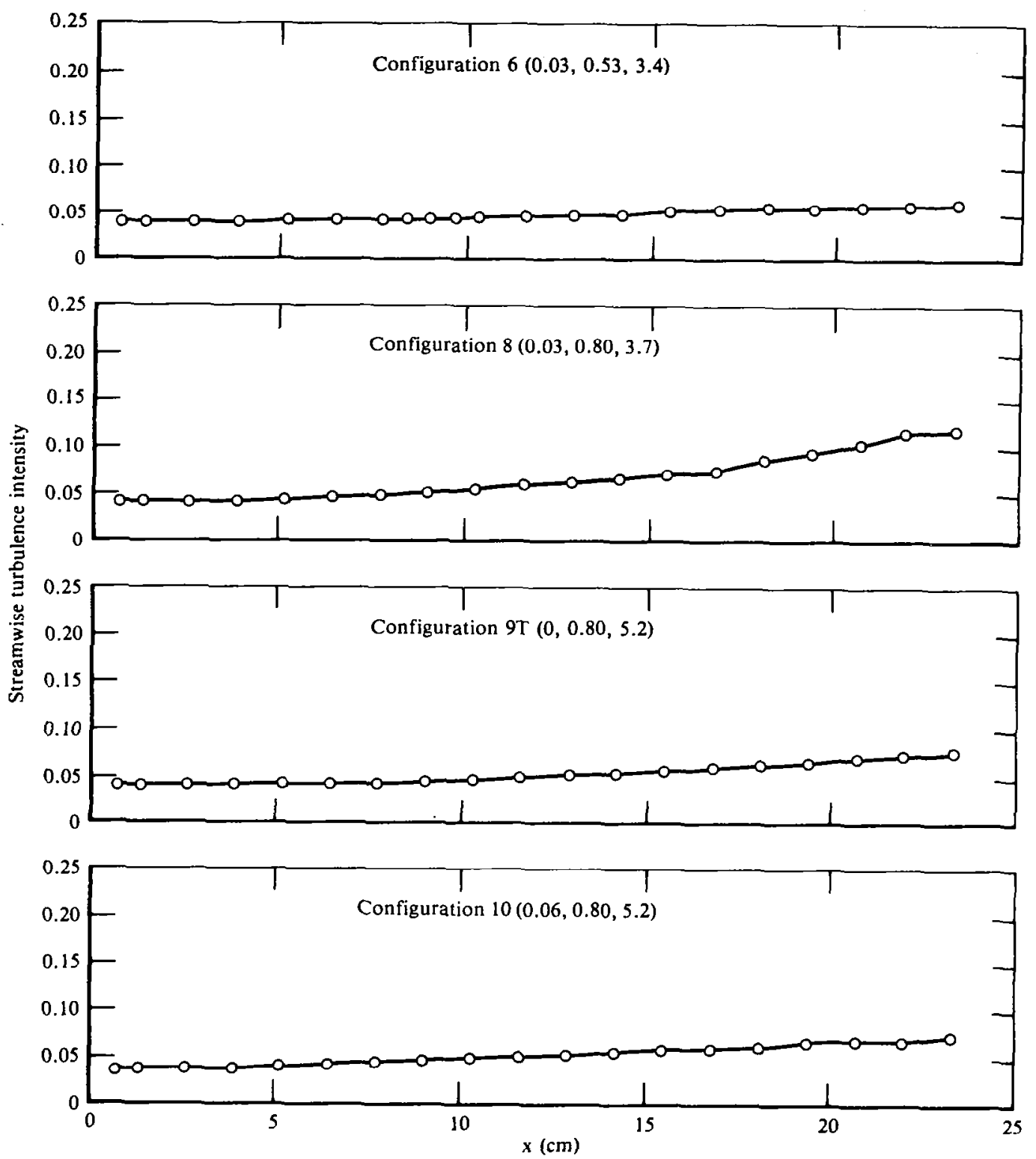

Figure B8. Streamwise turbulence intensity profiles at a nozzle pressure ratio of 1.6. 

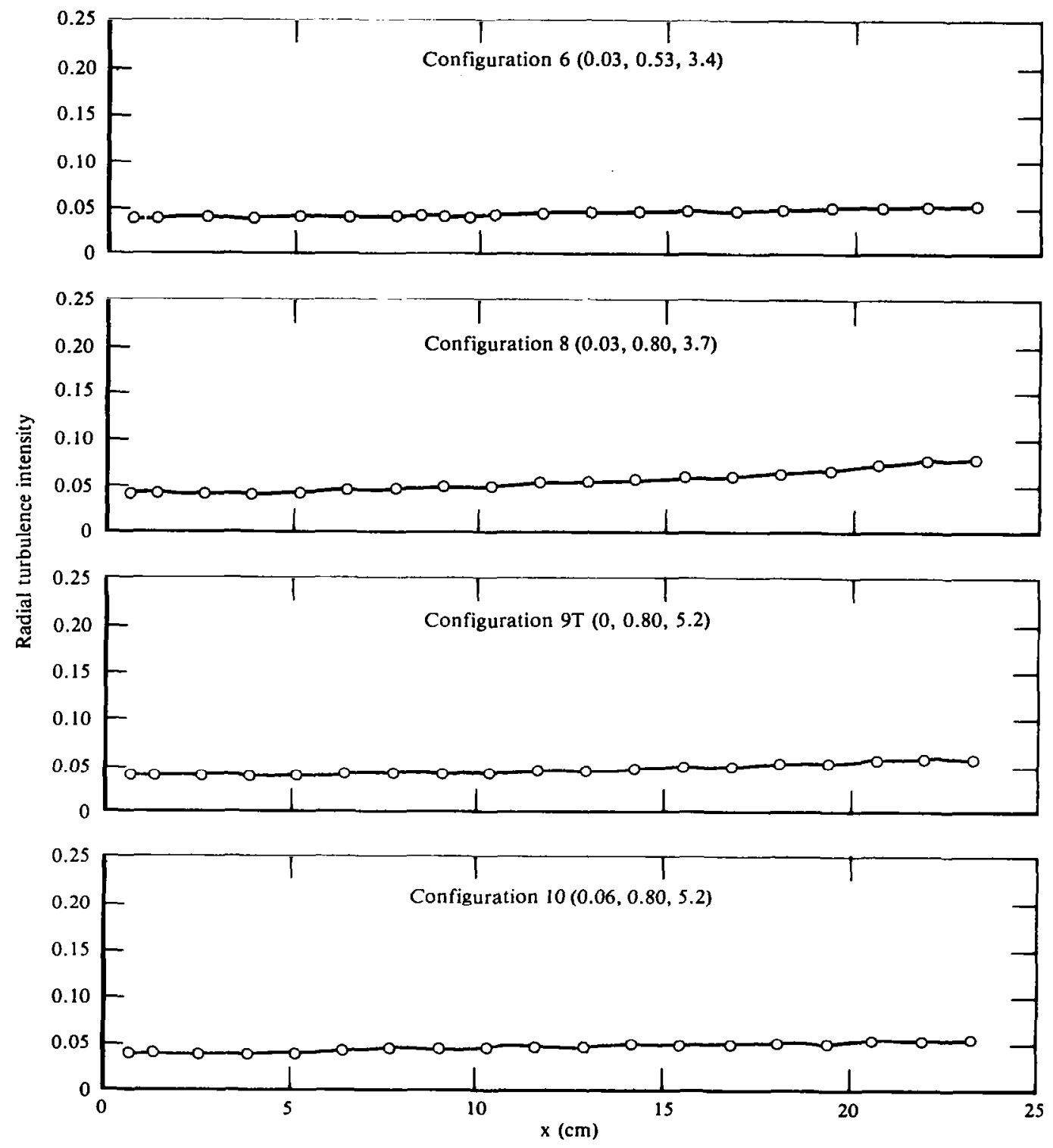

Figure B9. Radial turbulence profiles at a nozzle pressure ratio of 1.6. 

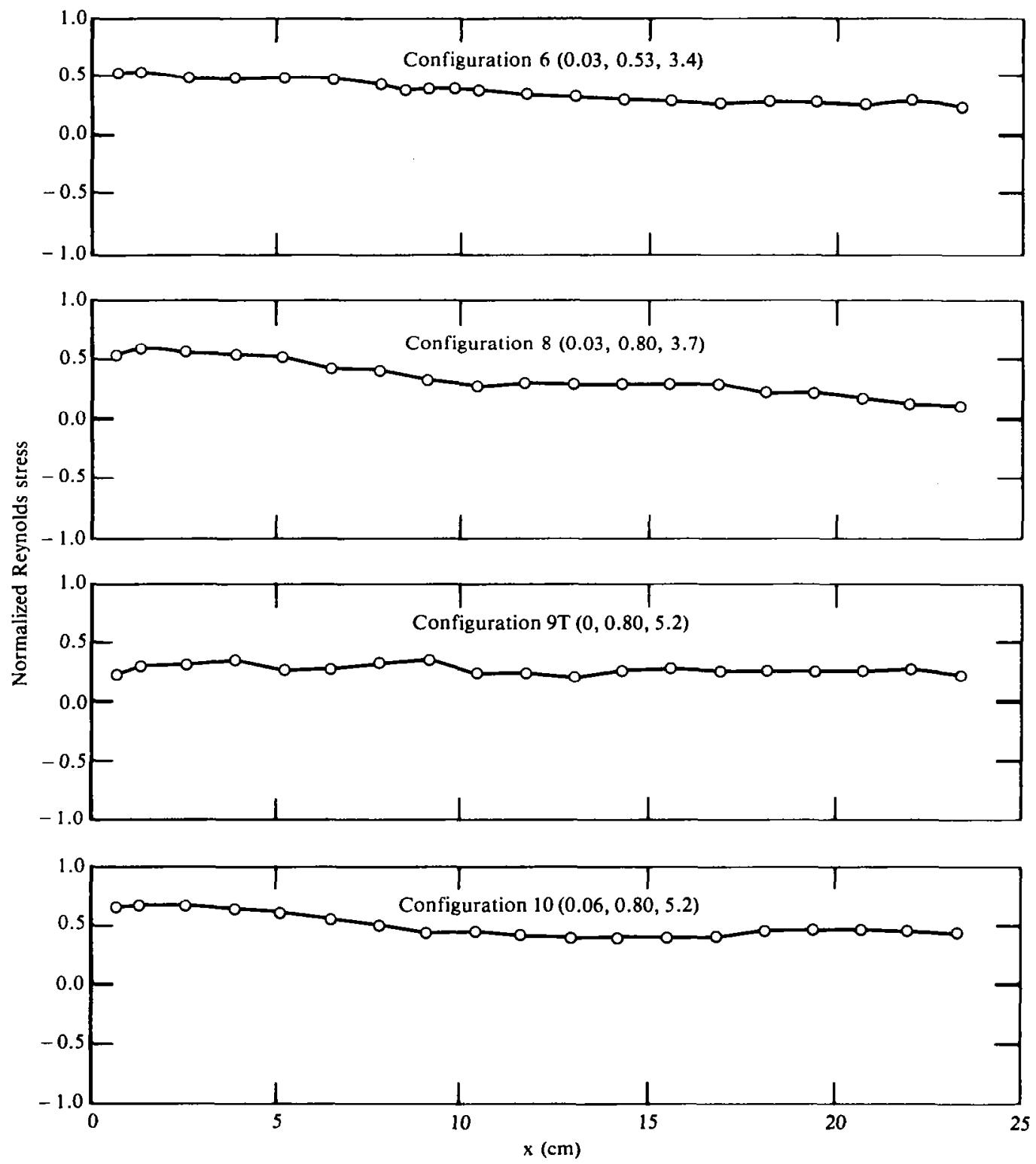

Figure B10. Normalized Reynolds stress profiles at a nozzle pressure ratio of 1.6 . 

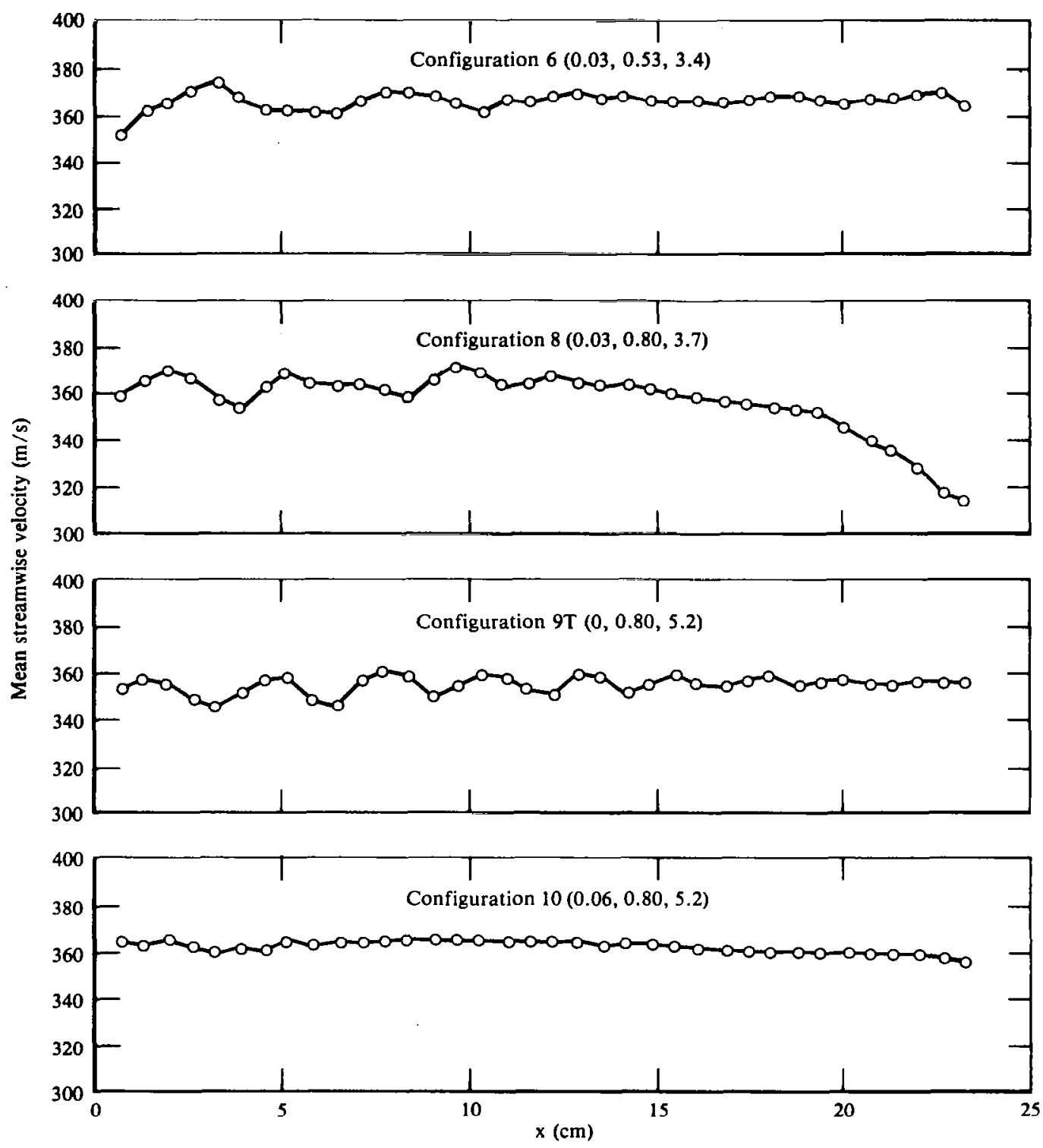

Figure B11. Mean streamwise velocity profiles at a nozzle pressure ratio of 2.2. 

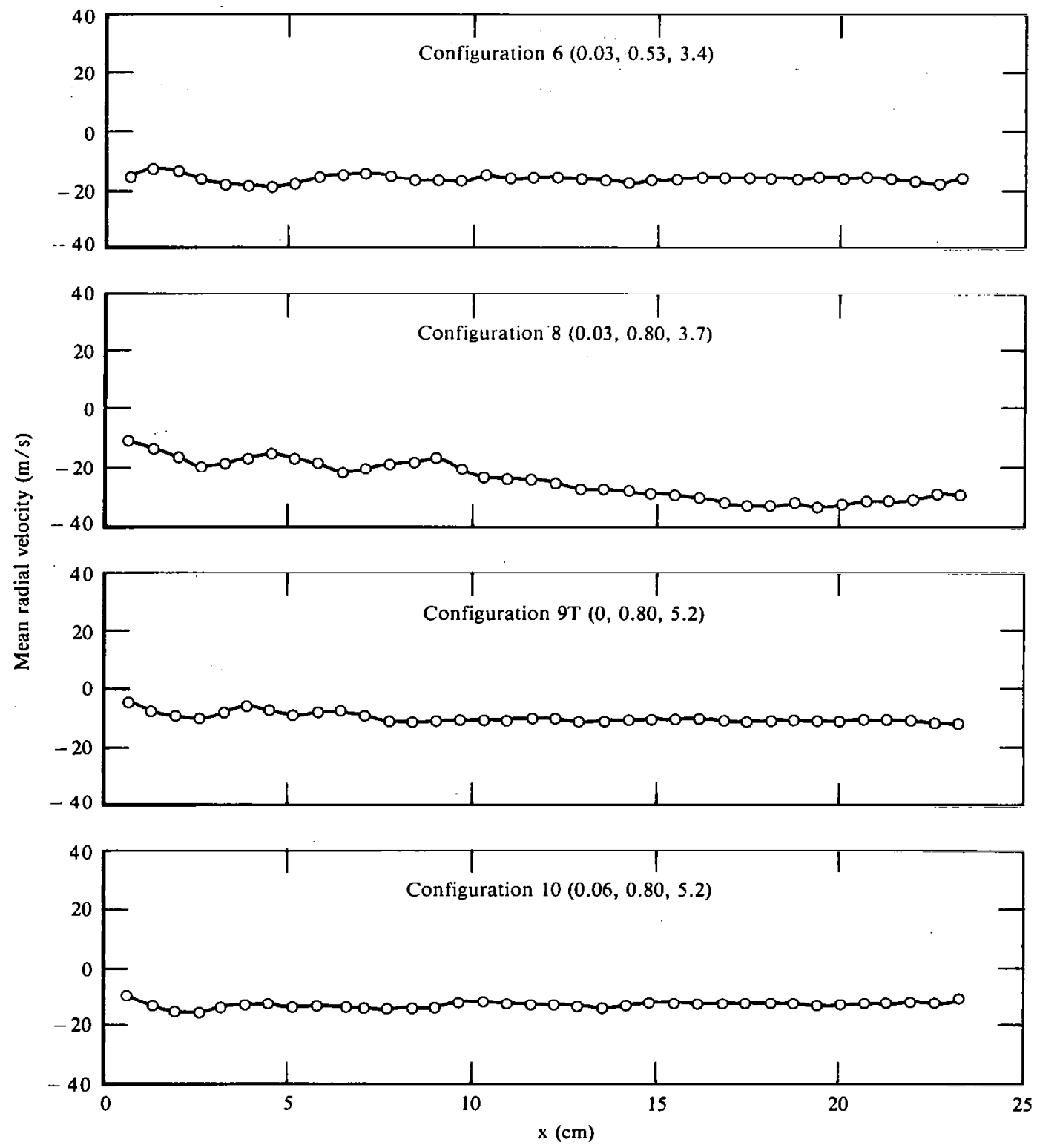

Figure B12. Mean radial velocity profiles at a nozzle pressure ratio of 2.2. 

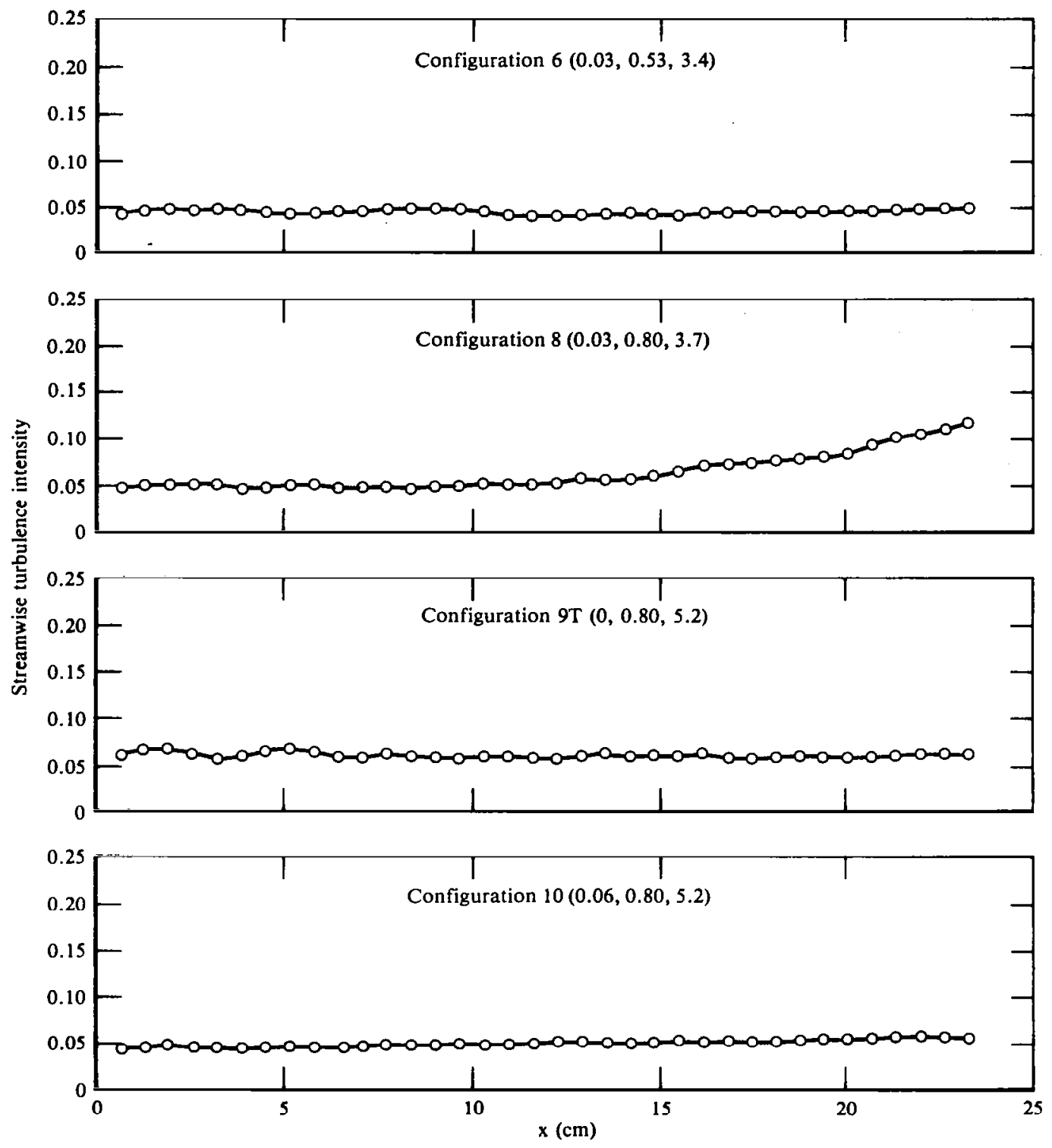

Figure B13. Streamwise turbulence intensity profiles at a nozzle pressure ratio of $\mathbf{2 . 2}$. 

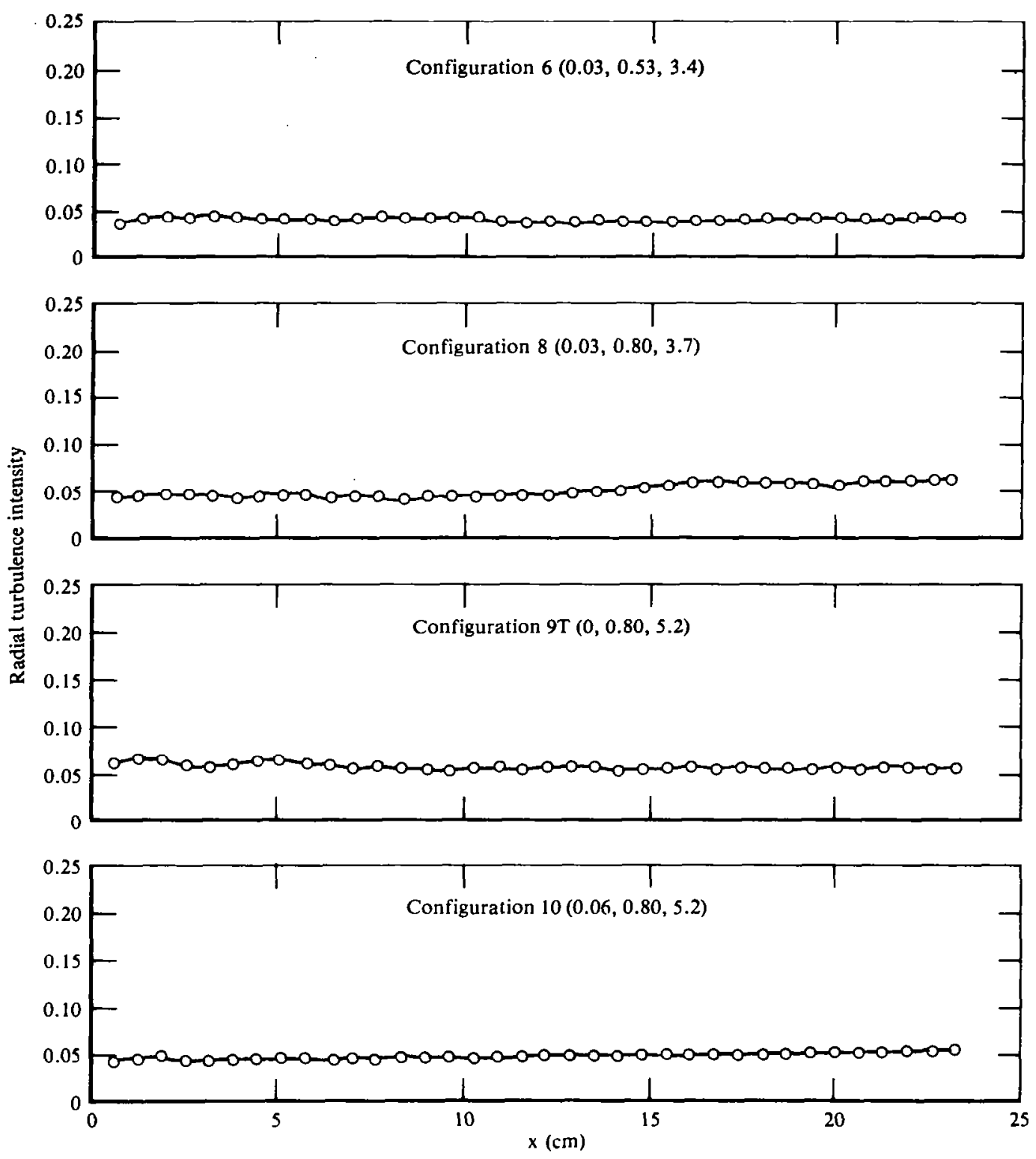

Figure B14. Radial turbulence intensity velocity profiles at a nozzle pressure ratio of 2.2 . 

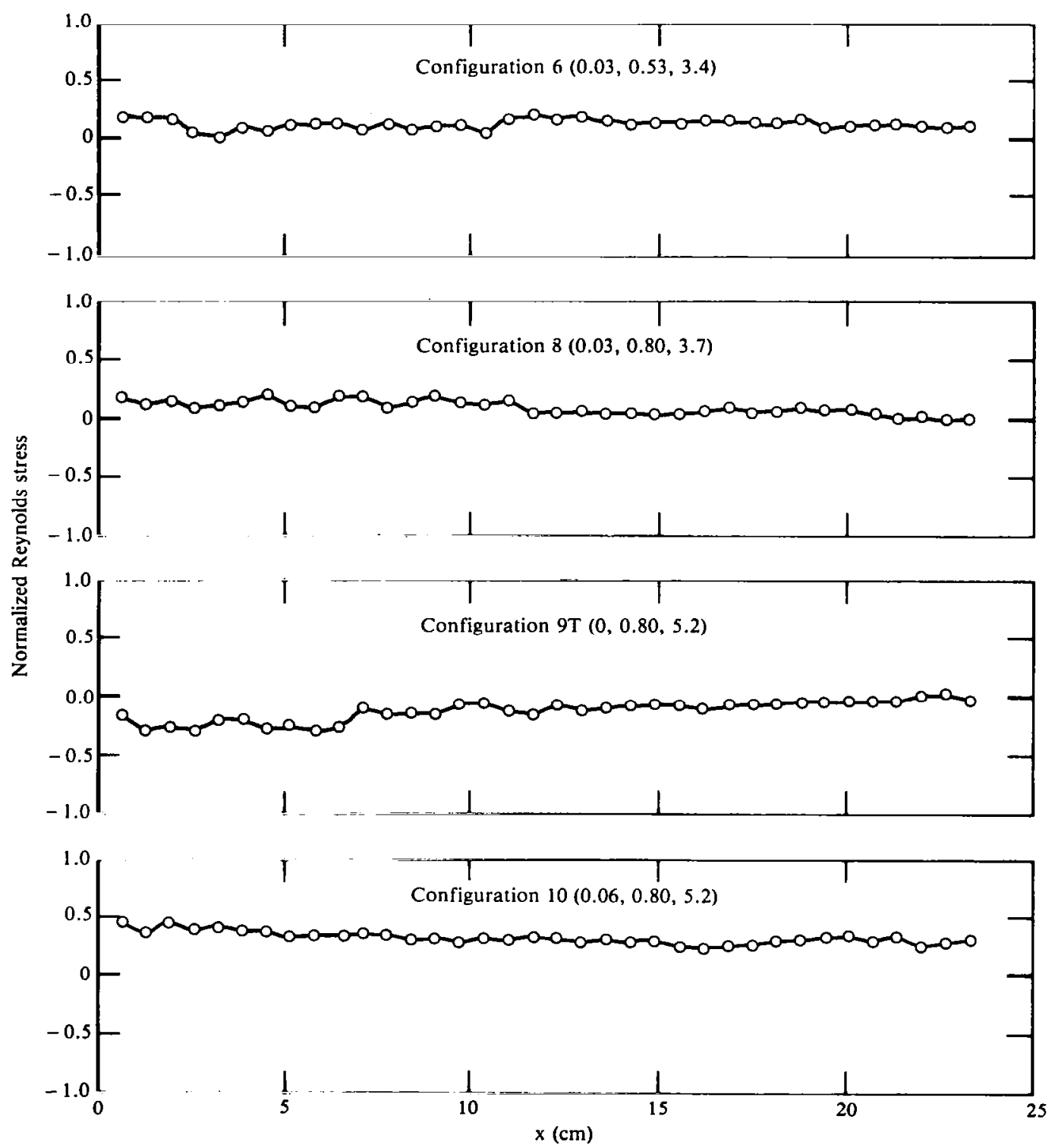

Figure B15. Normalized Reynolds stress profiles at a nozzle pressure ratio of 2.2 . 


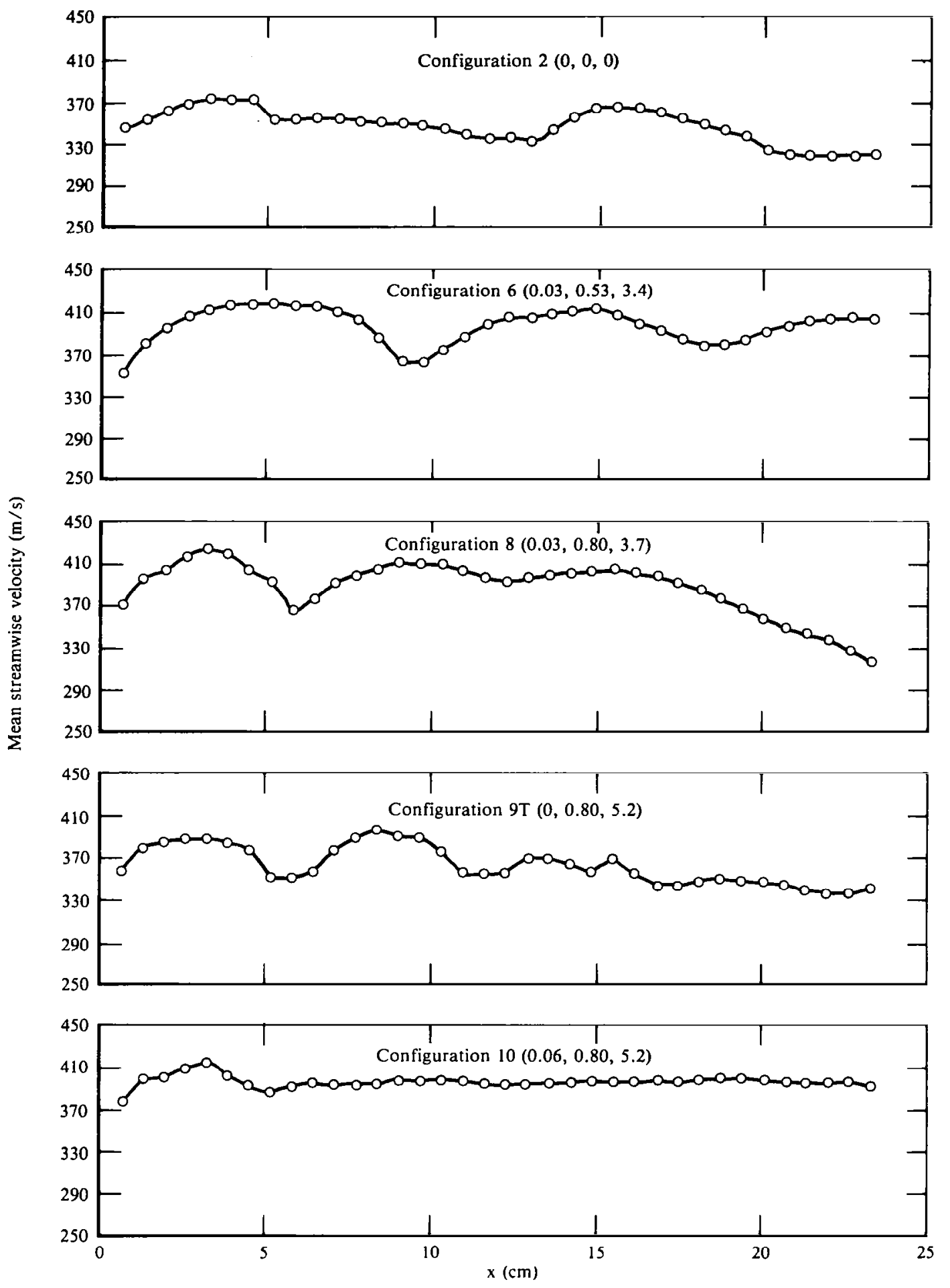

Figure B16. Mean streamwise velocity profiles at a nozzle pressure ratio of 2.8 . 

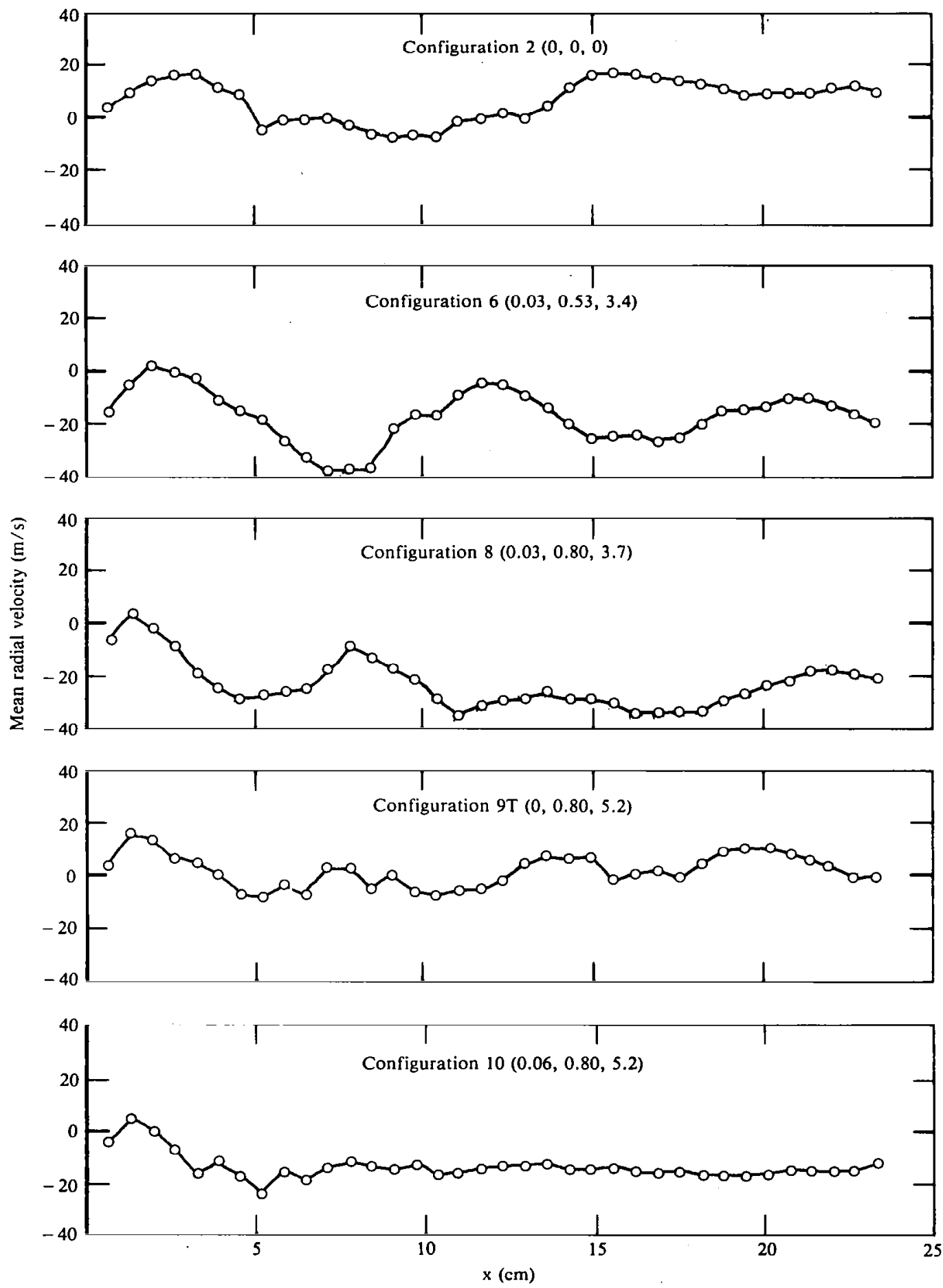

Figure B17. Mean radial velocity profiles at a nozzle pressure ratio of 2.8 . 

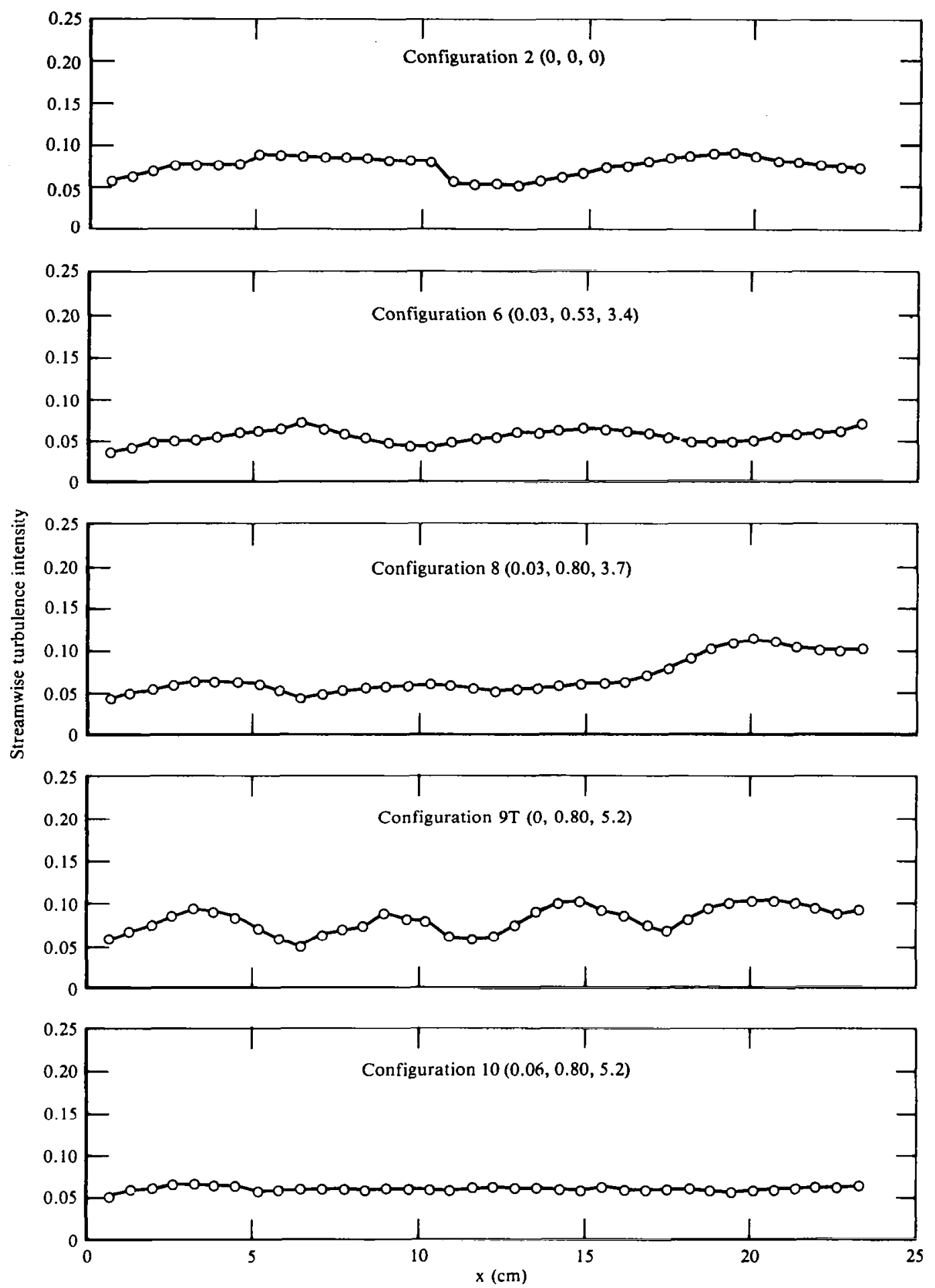

Figure B18. Streamwise turbulence intensity profiles at a nozzle pressure ratio of 2.8 . 

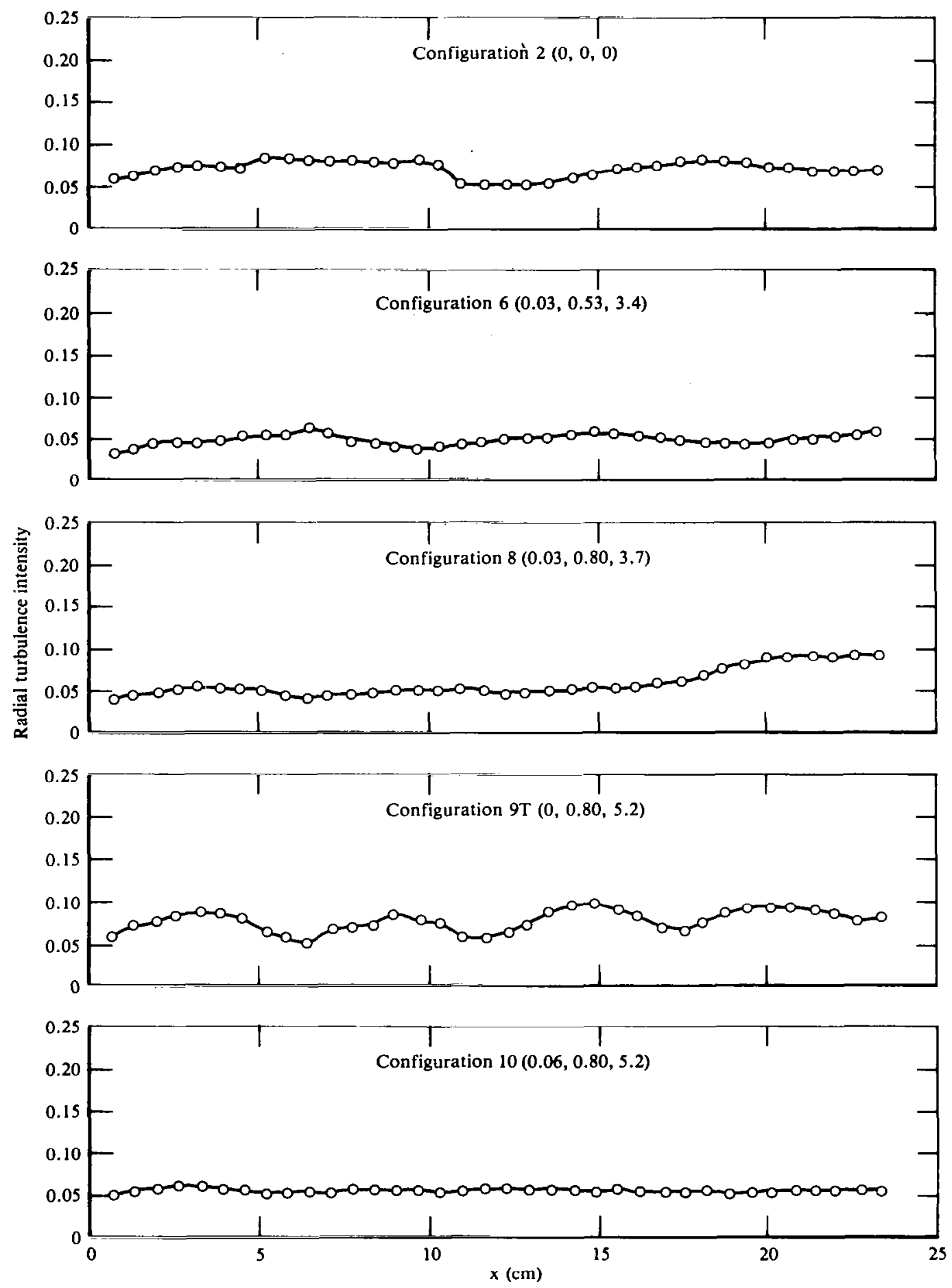

Figure B19. Radial turbulence intensity profiles at a nozzle pressure ratio of 2.8 . 

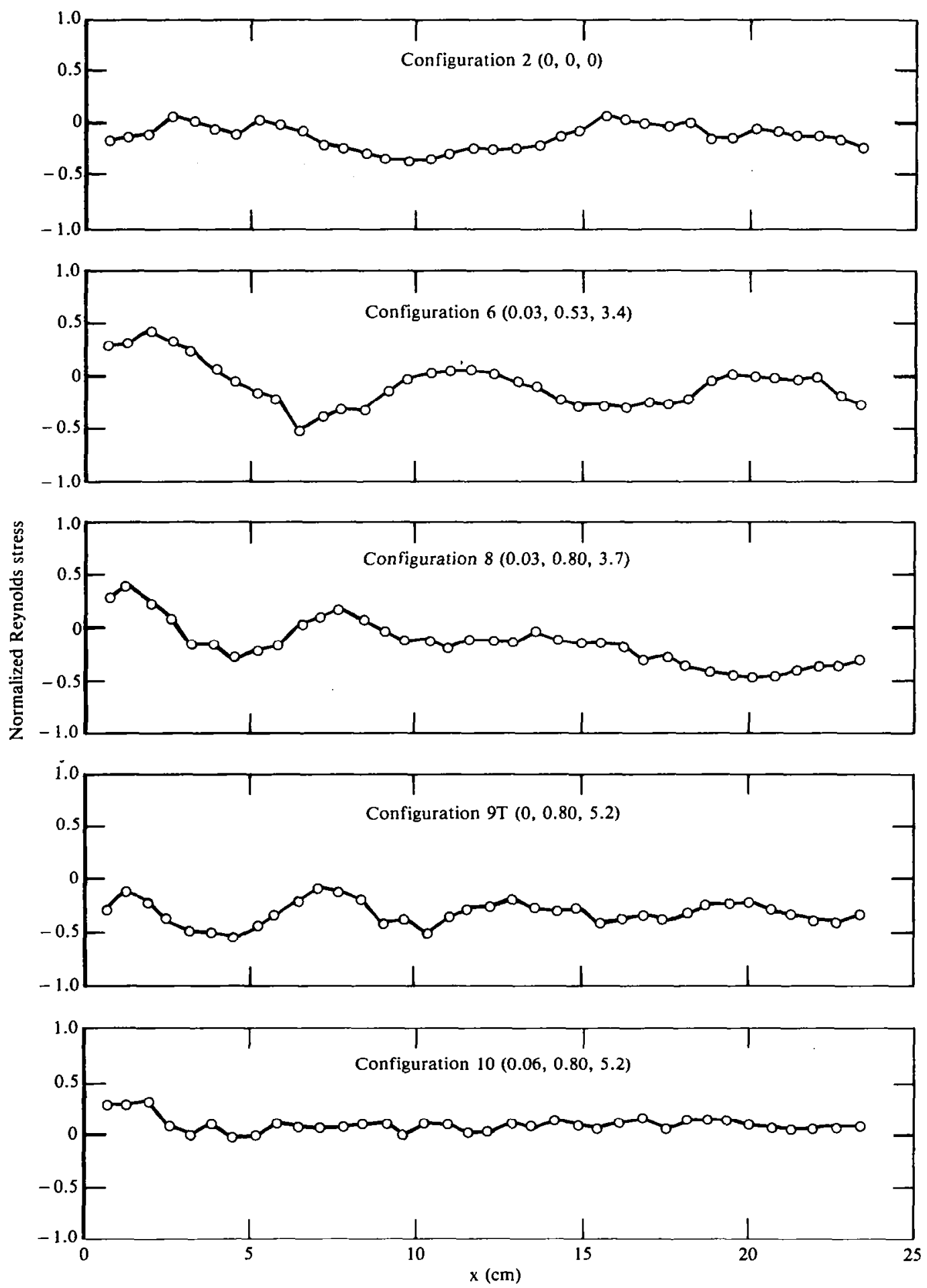

Figure B20. Normalized Reynolds stress profiles at a nozzle pressure ratio of 2.8 . 


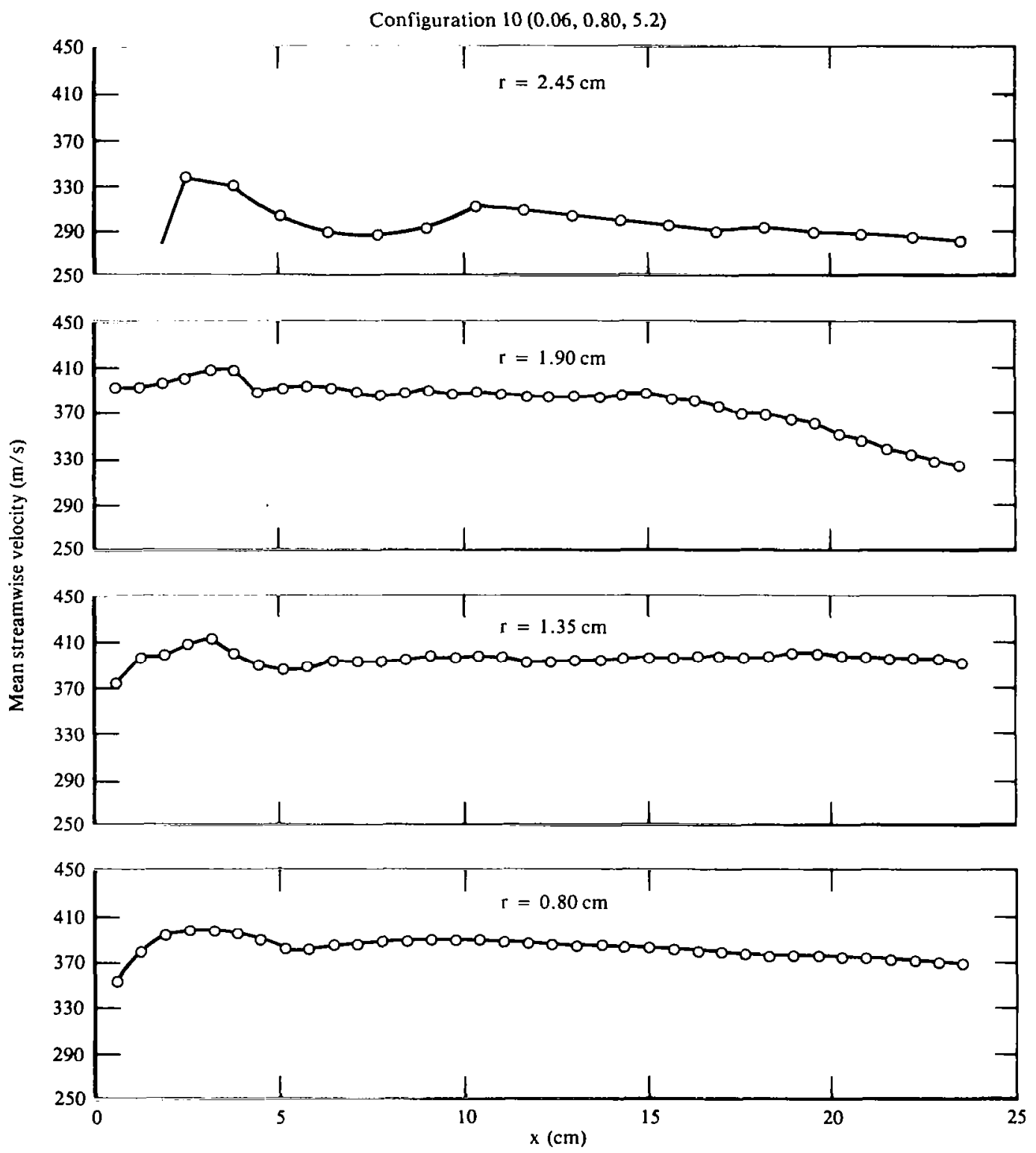

Figure B21. Radial variation of mean streamwise velocity profiles at a nozzle pressure ratio of 2.8 . 


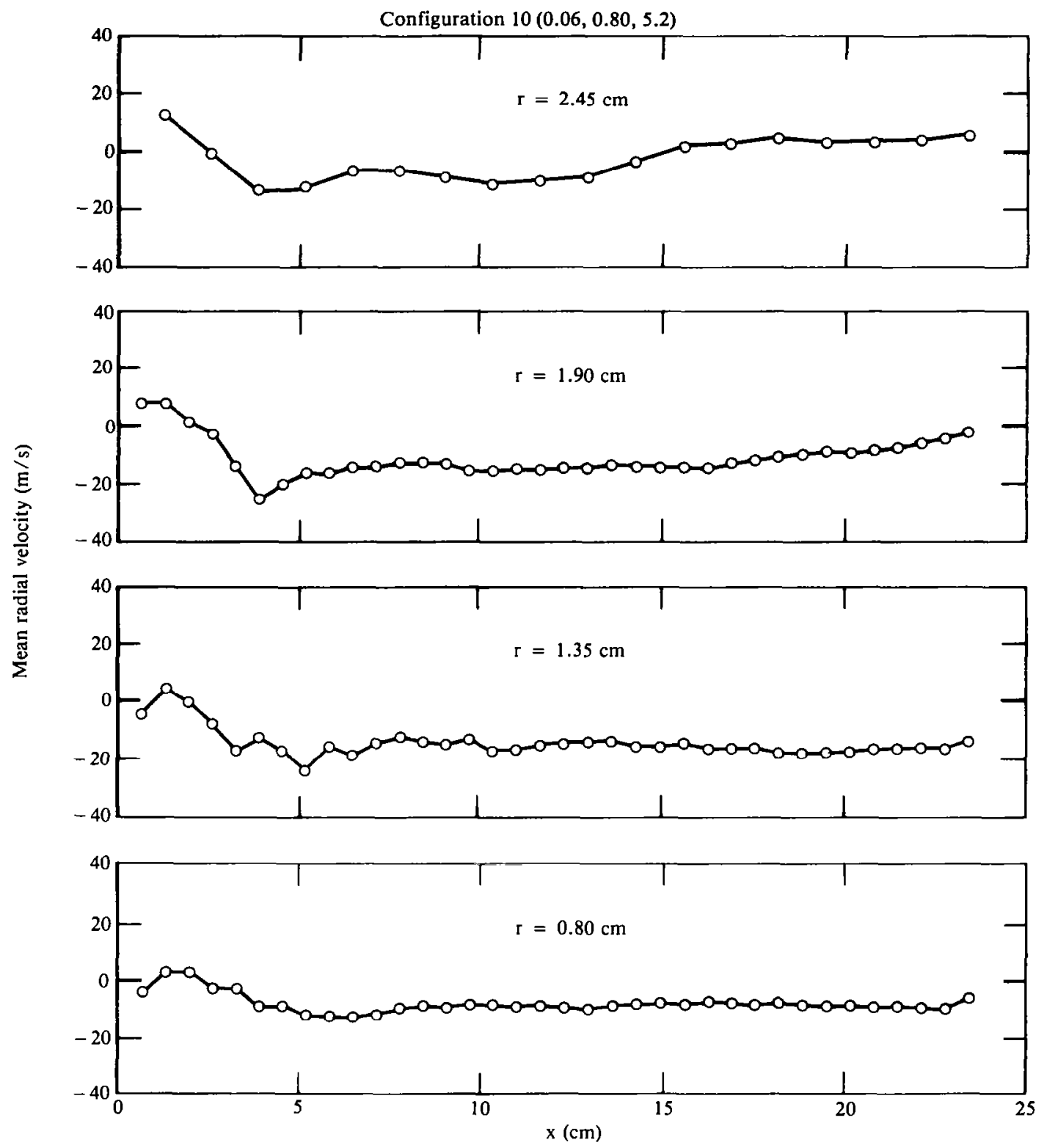

Figure B22. Radial variation of mean radial velocity profiles at a nozzle pressure ratio of 2.8 . 

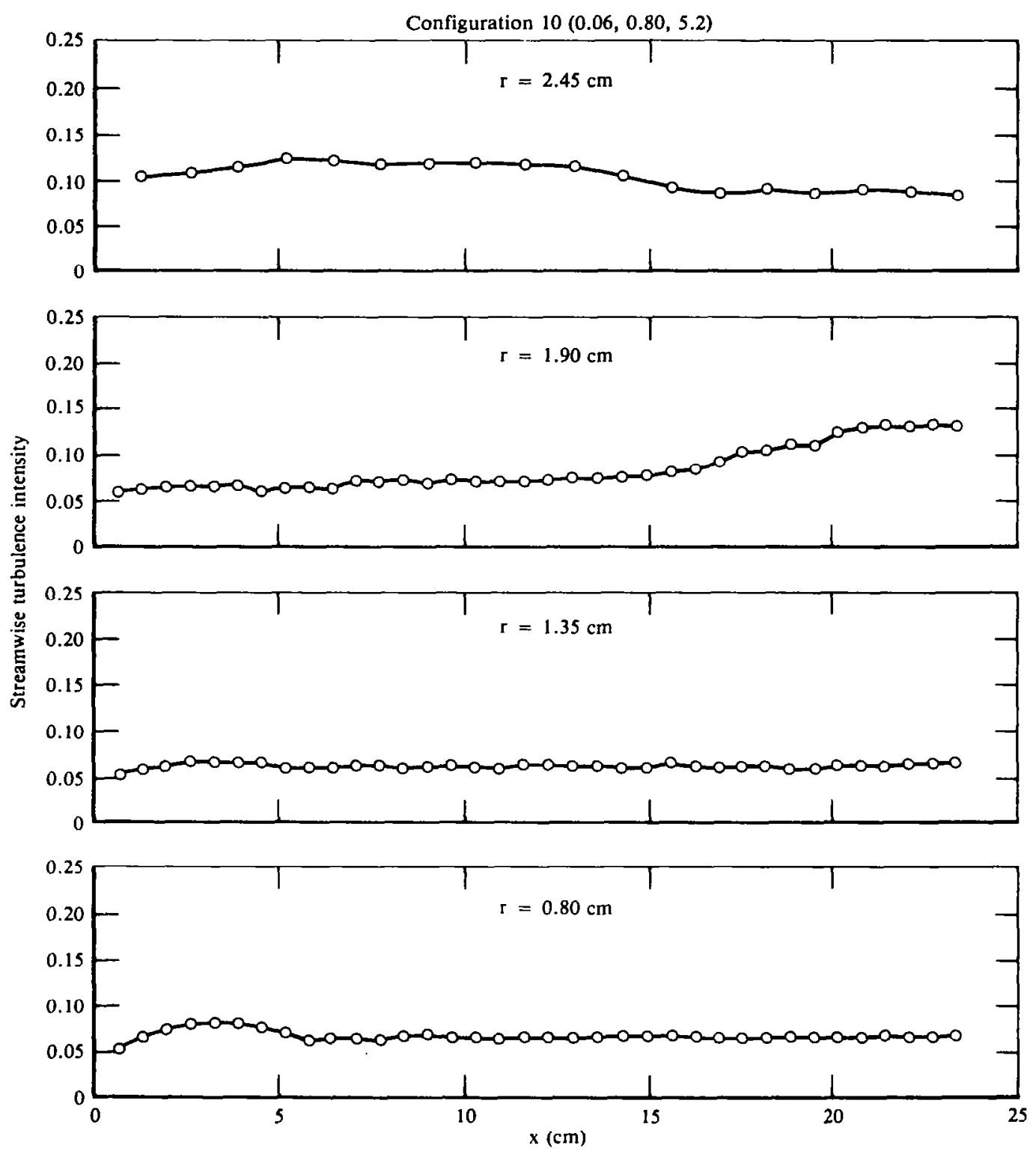

Figure B23. Kadial variation of streamwise turbulence intensity profiles at a nozzle pressure ratio of 2.8 . 

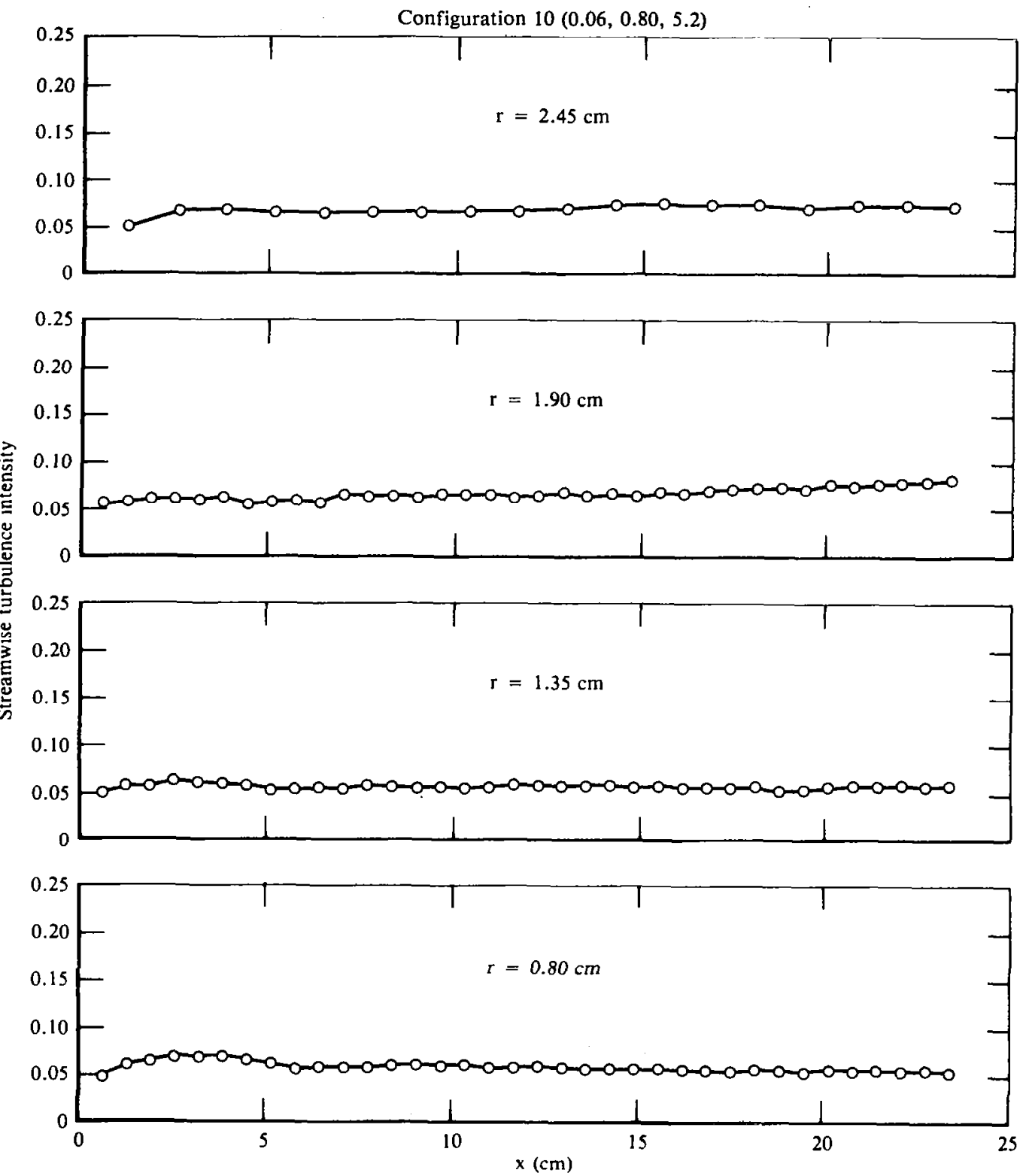

Figure B24. Radial variation of radial turbulence intensity profiles at a nozzle pressure ratio of 2.8 . 


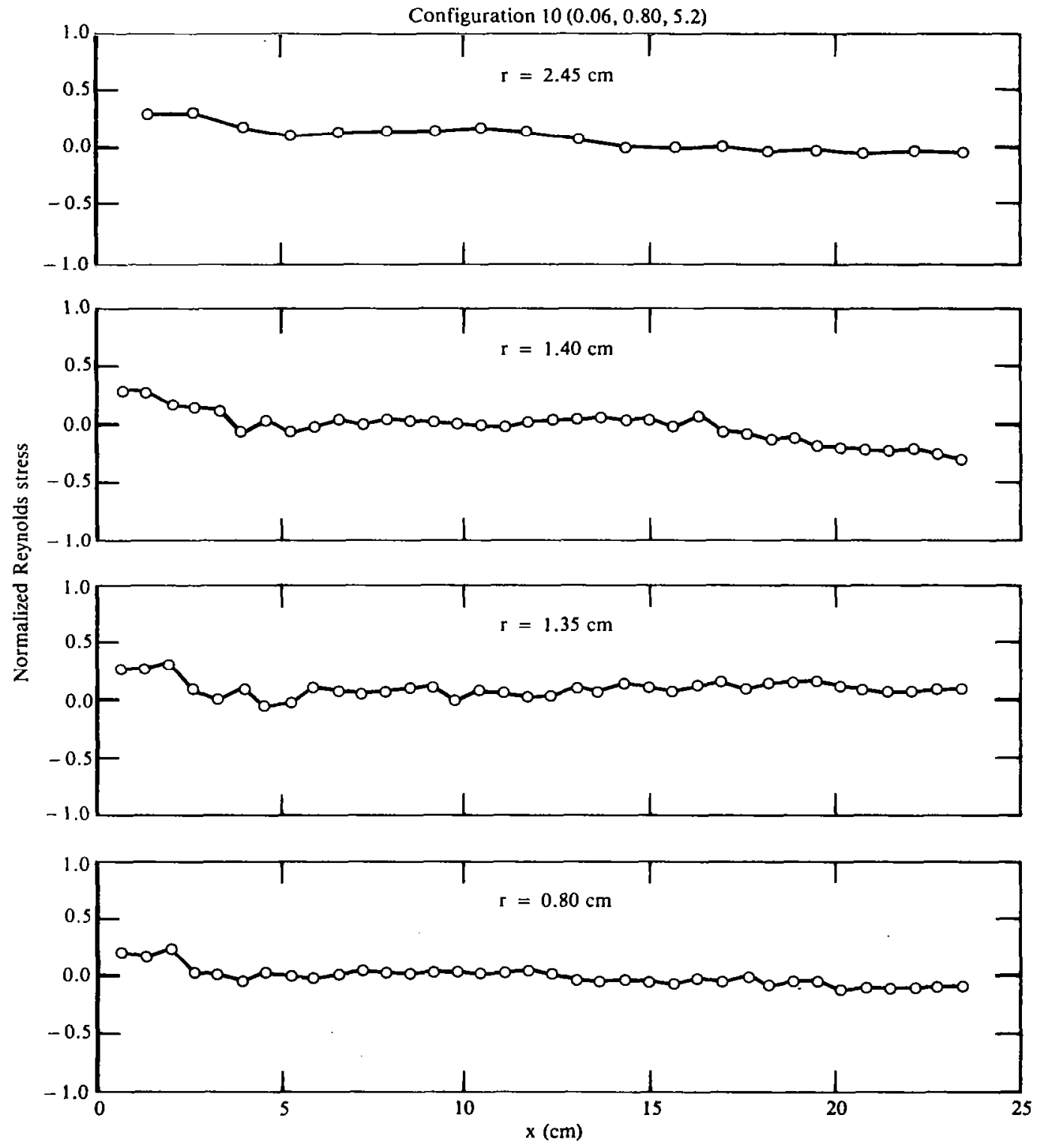

Figure B25. Radial variation of normalized Reynolds stress profiles at a pressure ratio of 2.8 . 


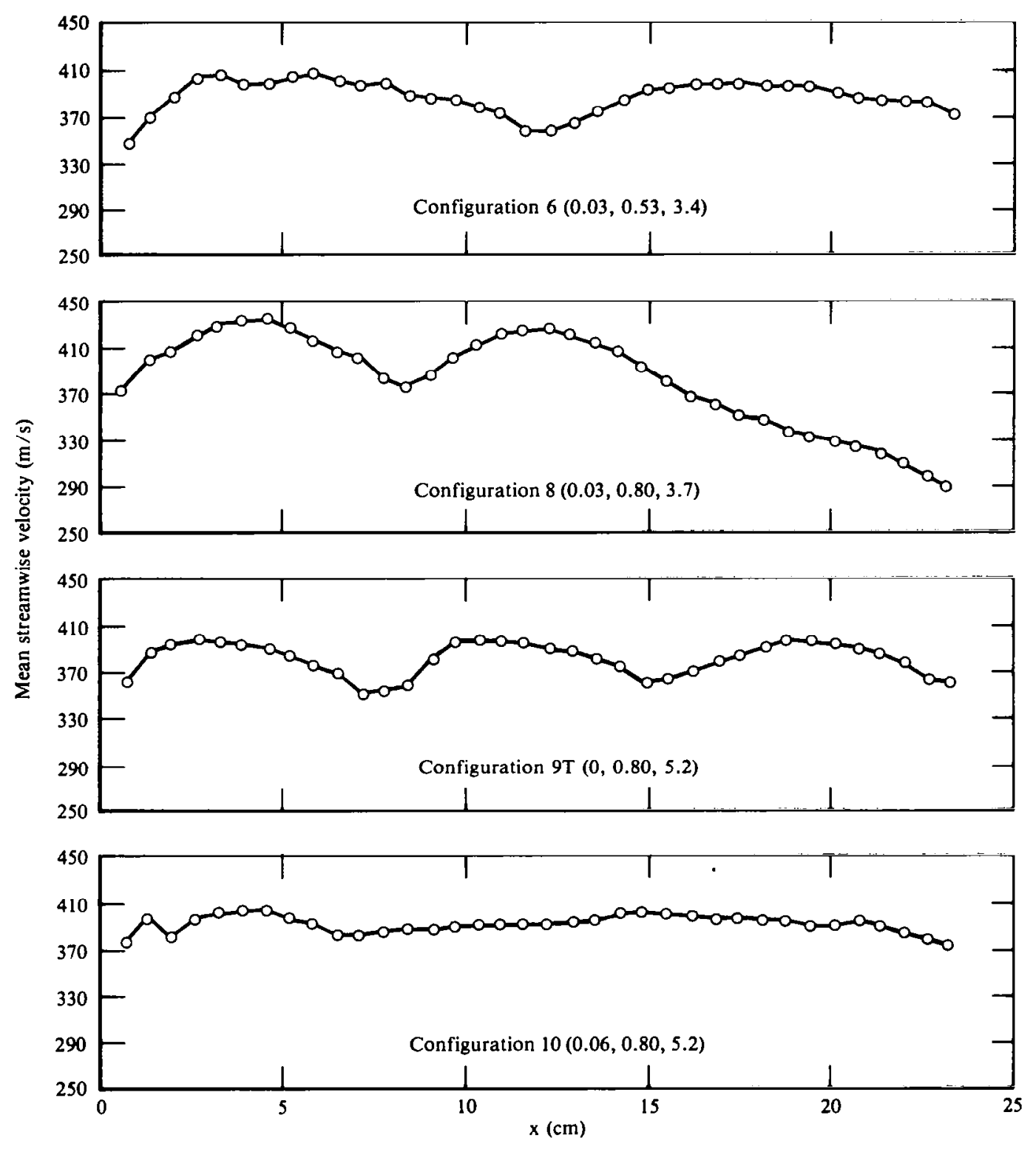

Figure B26. Mean streamwise velocity profiles at a nozzle pressure ratio of 3.4. 

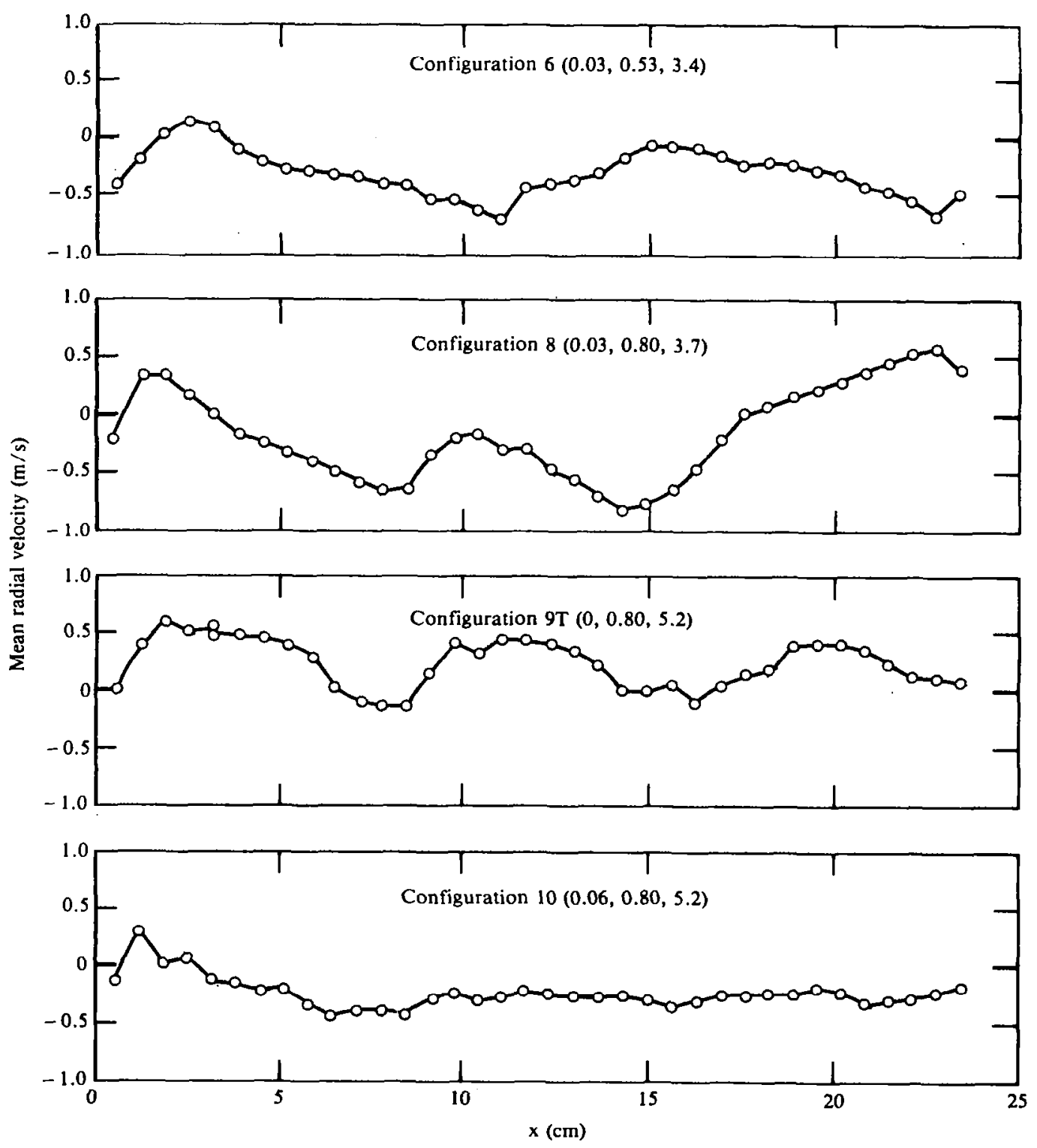

Figure B27. Mean radial velocity profiles at a nozzle pressure ratio of 3.4 . 

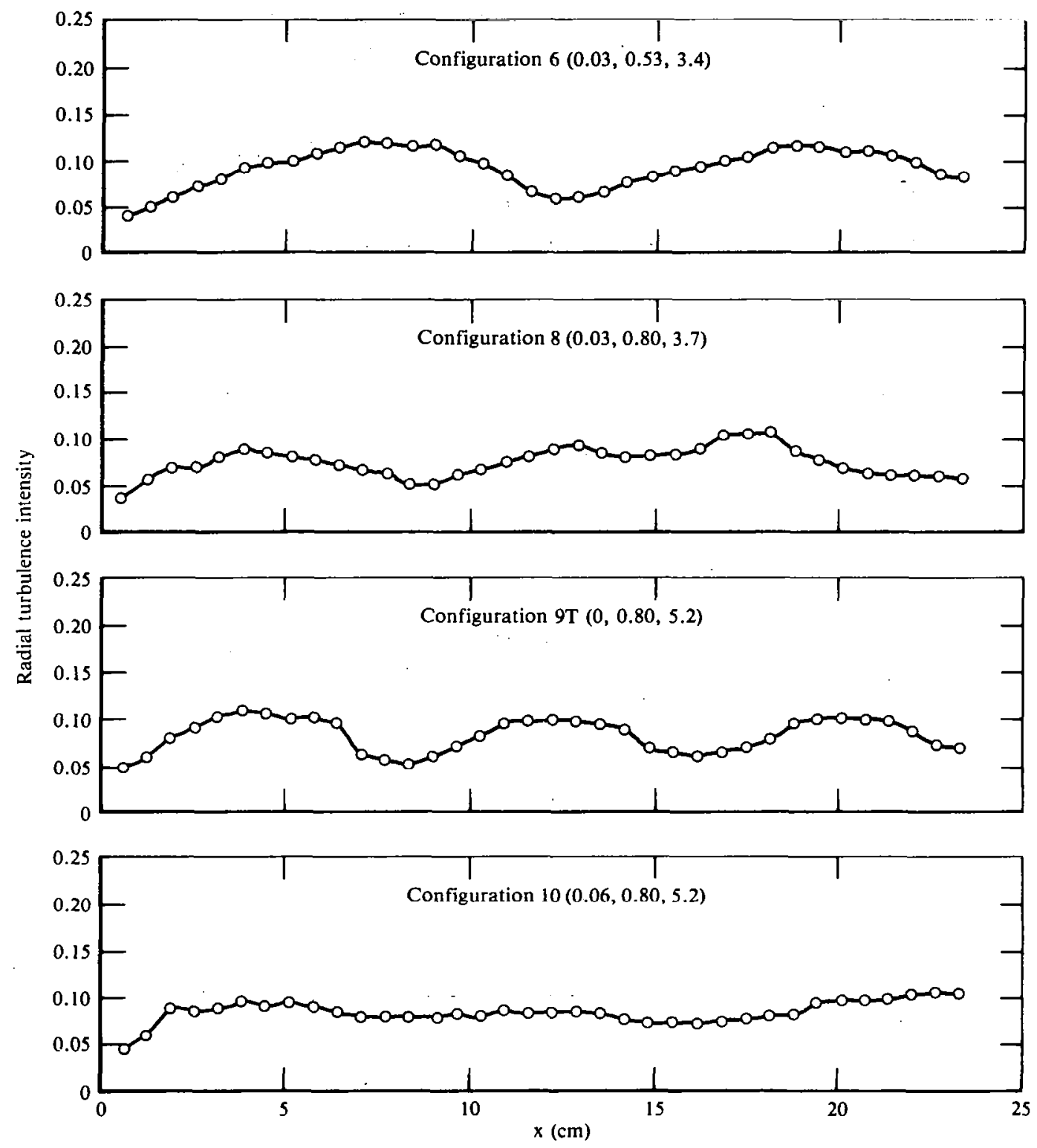

Figure B28. Streamwise turbulence intensity profiles at a nozzle pressure ratio of 3.4. 


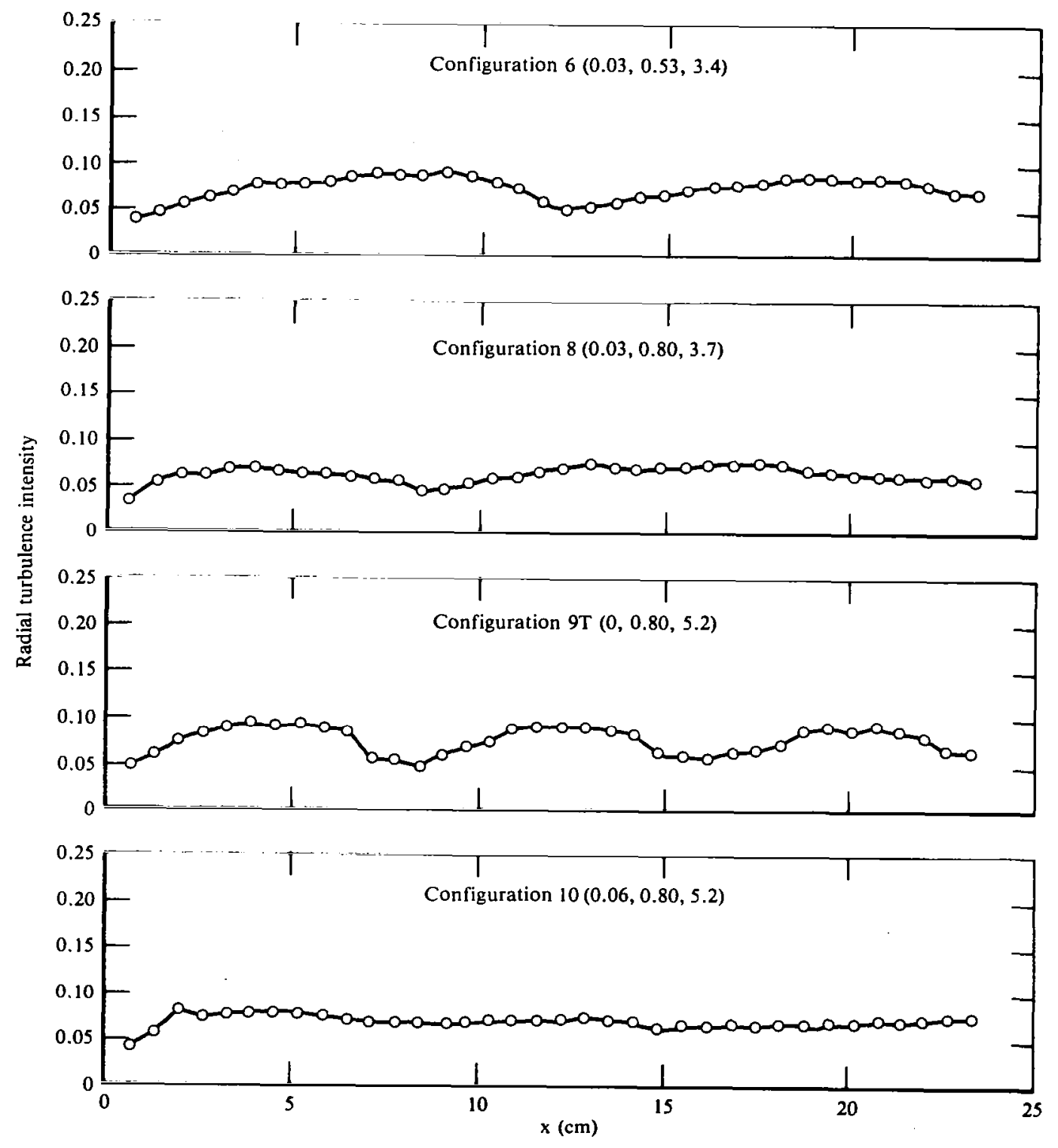

Figure B29. Radial turbulence intensity profiles at a nozzle pressure ratio of 3.4 . 


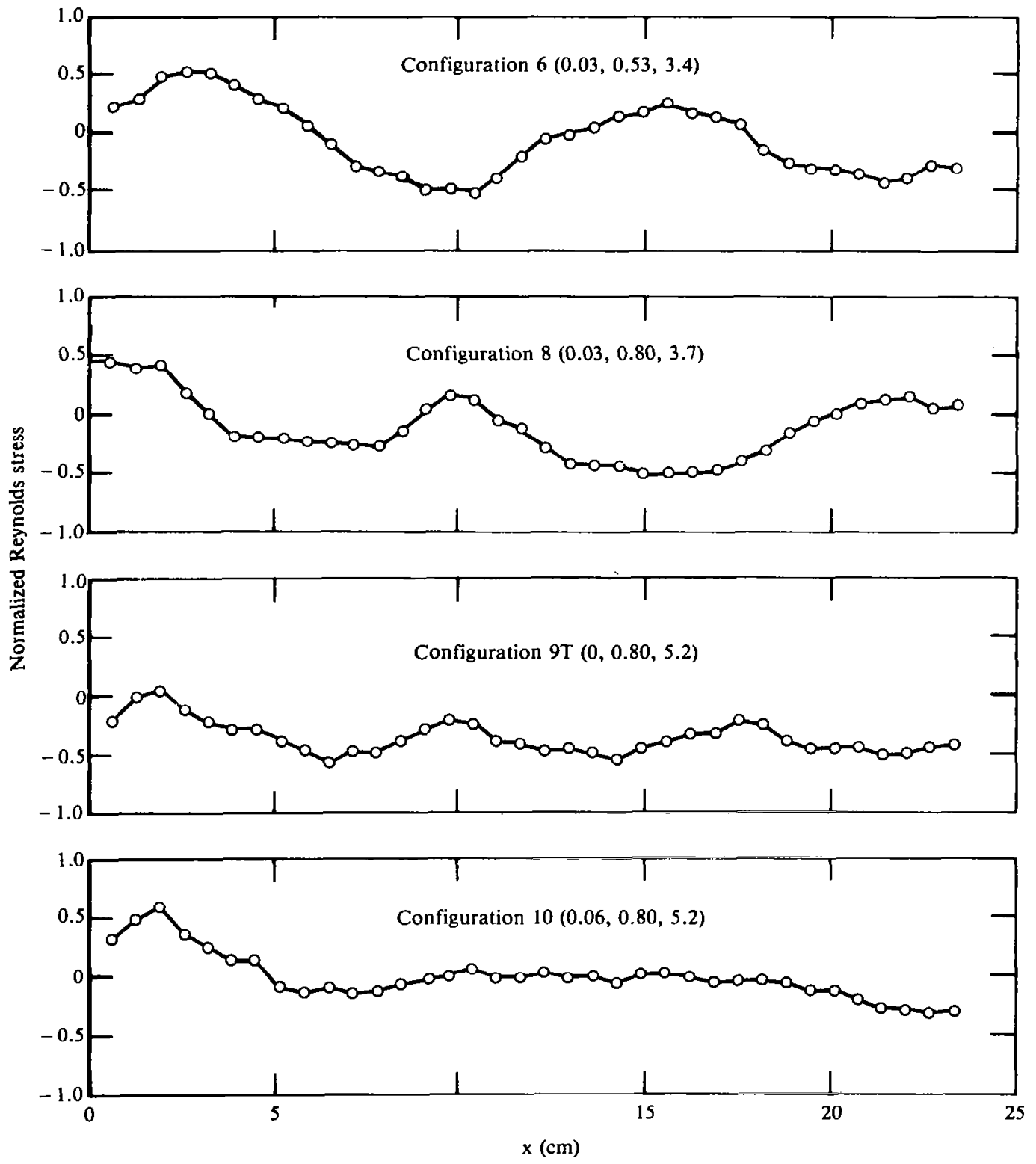

Figure B30. Normalized Reynolds stress profiles at a nozzle pressure ratio of 3.4. 

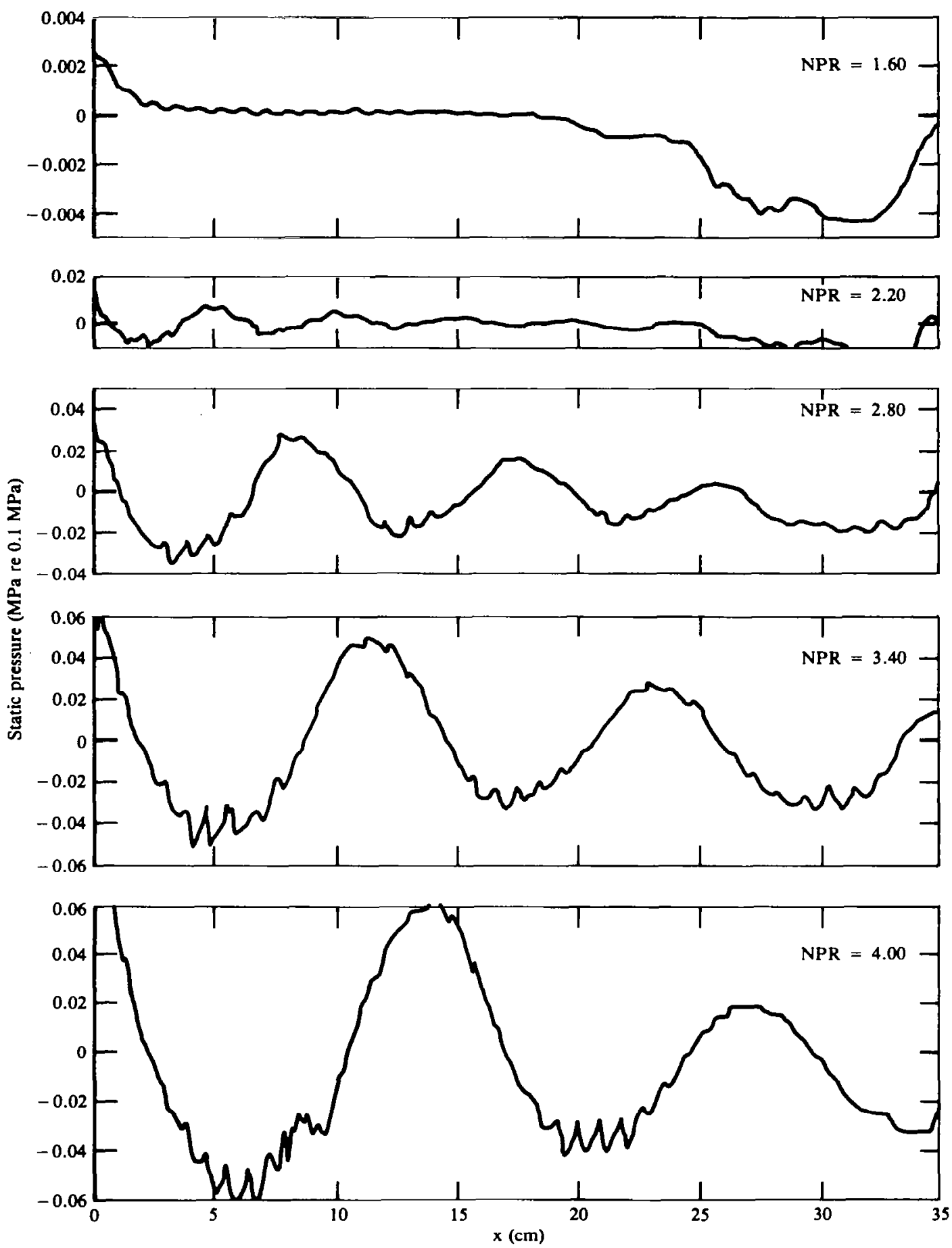

Figure B31. Streamwise variation of static pressure $2.5 \mathrm{~mm}$ above plug surface for configuration $6(0.03,0.53,3.4)$. 

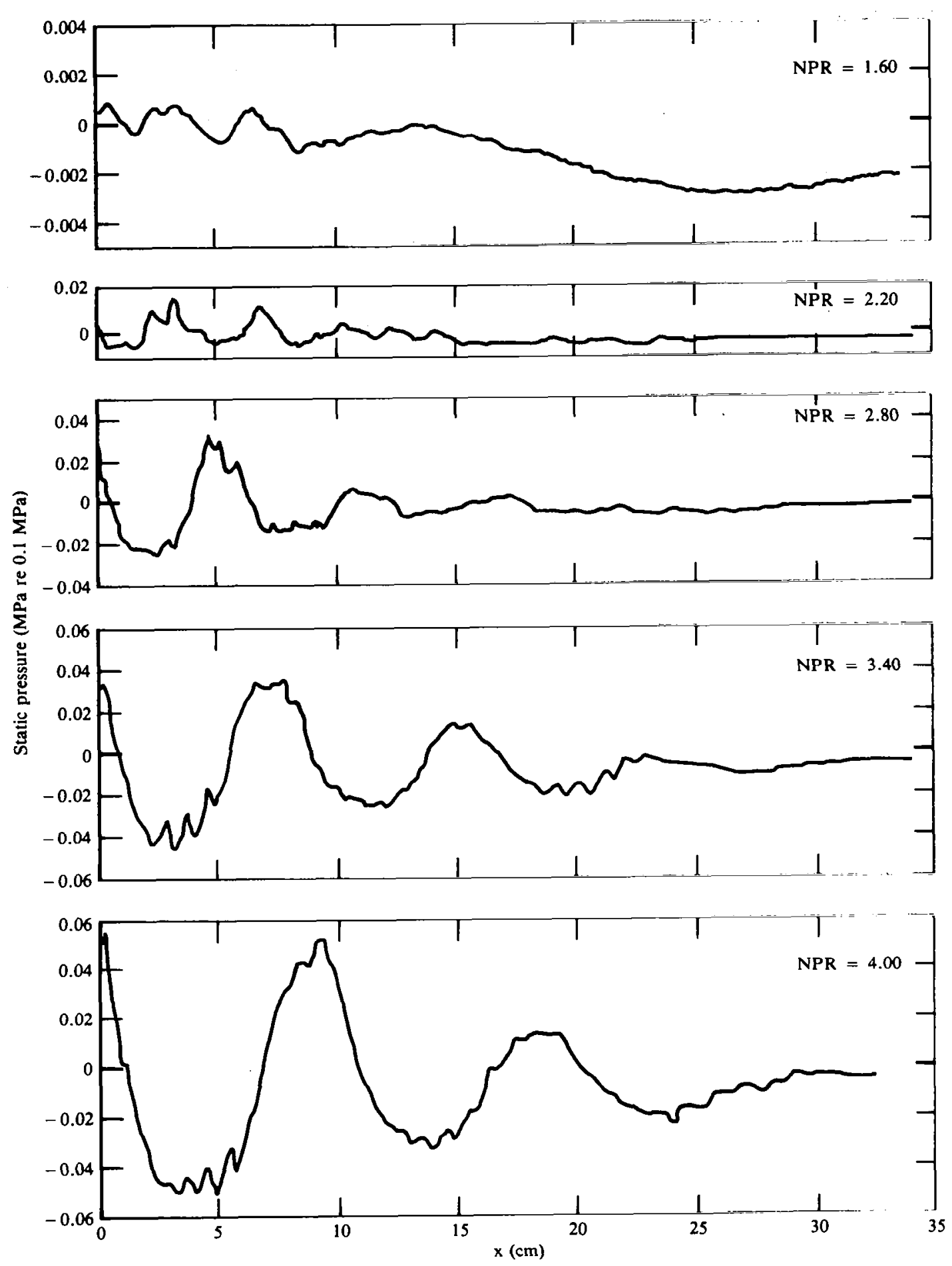

Figure B32. Streamwise variation of static pressure $2.5 \mathrm{~mm}$ above plug surface for configuration $8(0.03,0.80,3.7)$. 

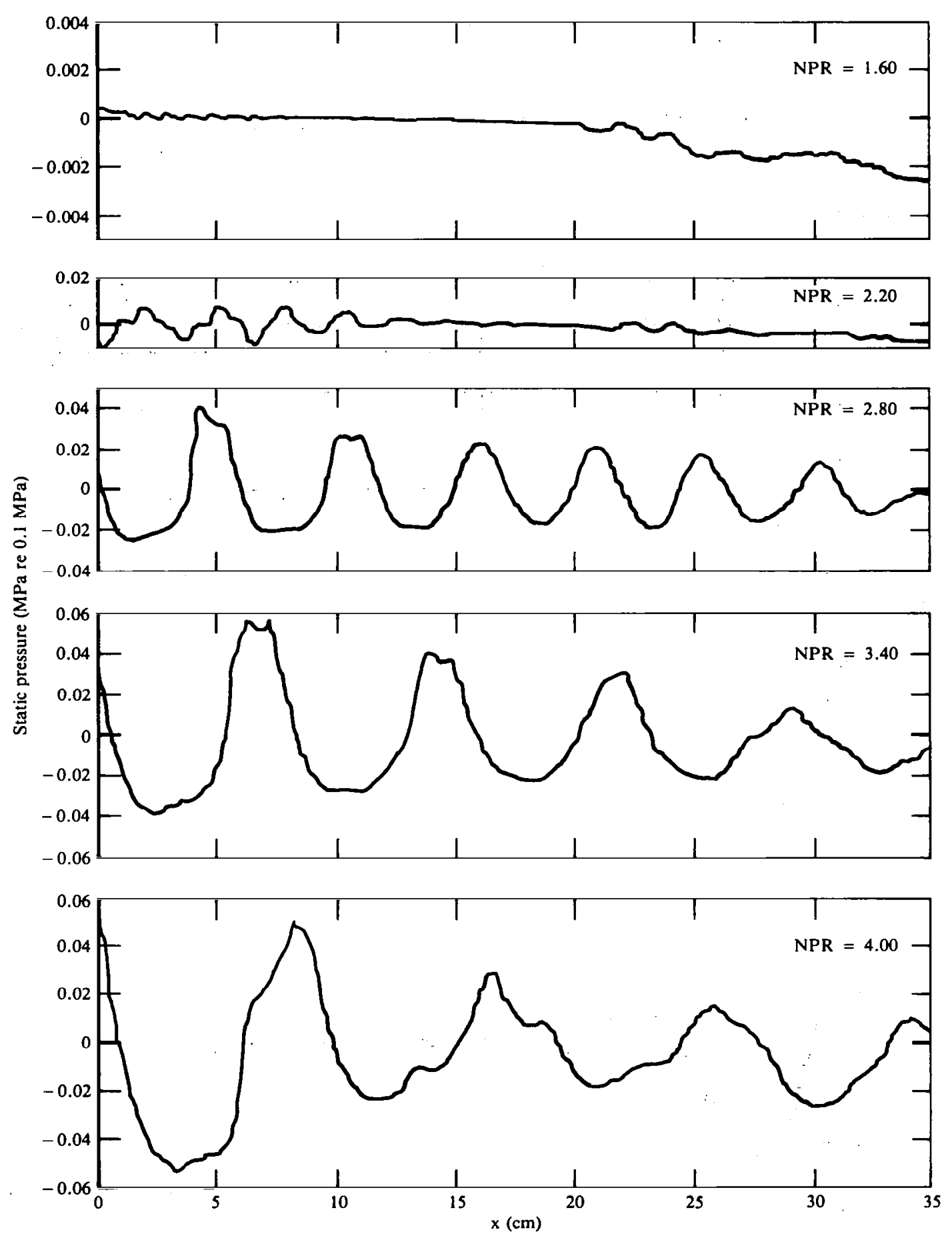

Figure B33. Streamwise variation of static pressure $2.5 \mathrm{~mm}$ above plug surface for configuration $9 T(0,0.80,5.2)$. 

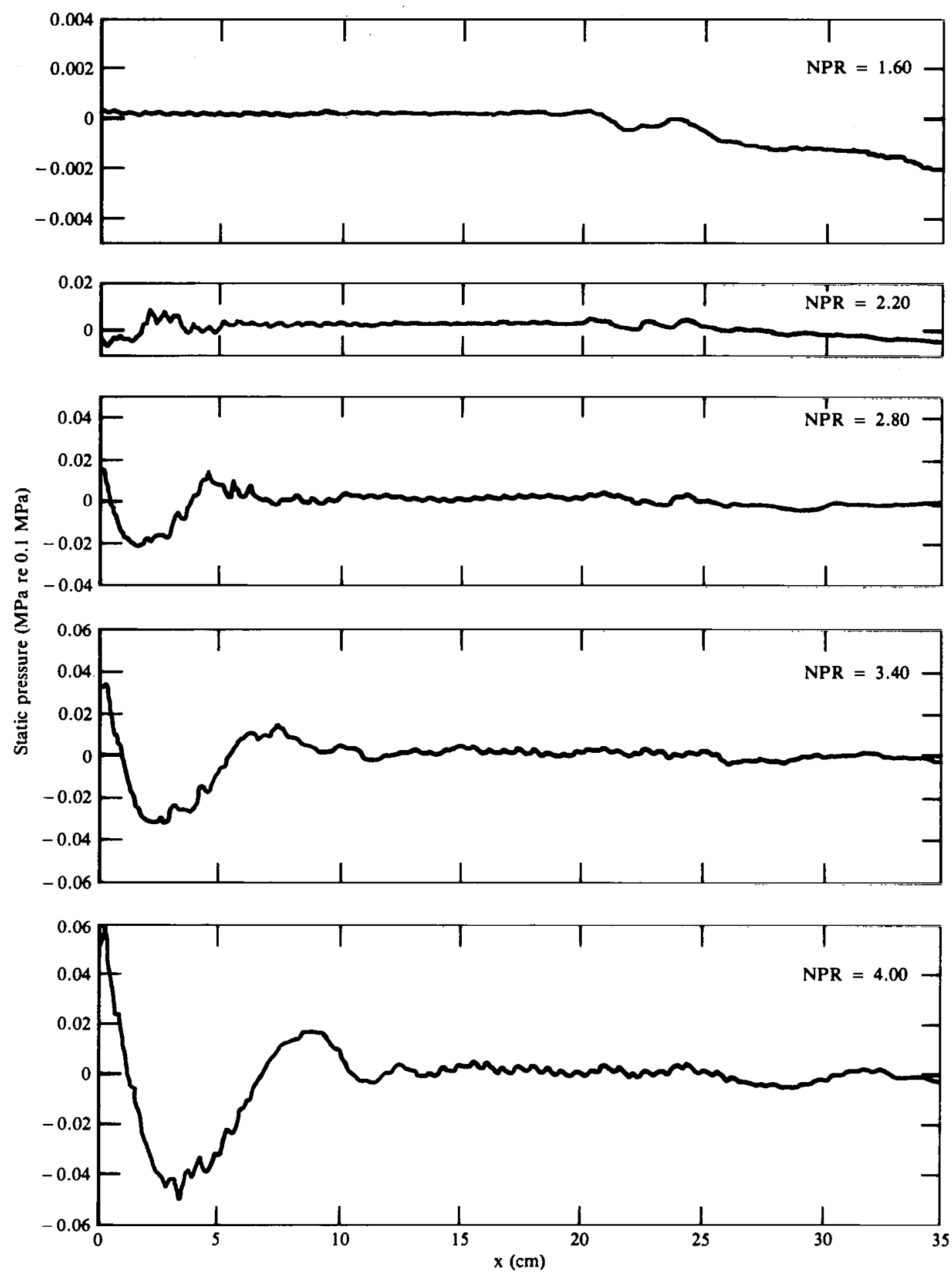

Figure B34. Streamwise variation of static pressure $2.5 \mathrm{~mm}$ above plug surface for configuration $10(0.06,0.80,5.2)$. 


\section{APPENDIX C}

THRUST EFFICIENCY

Since tradeoffs between noise reduction and thrust degradation are important, a model for the thrust loss mechanism has been developed. The thrust coefficient, $\mathrm{C}_{\mathrm{T}}$, should be as close as practical to 1.0 for best efficiency, but several known aerodynamlc phenomena serve to reduce it. Using simple models of these phenomena, the $C_{T}$ data in Tables 6 through 10 have been analyzed to form quantitive estimates of the significant thrust reduction mechanisms.

The thrust coefficient may be expressed as

$$
C_{T}=1-C_{T s}-C_{T n}-C_{T f}-C_{T P}-C_{T h} \text {, }
$$

where terms represent various loss mechanisms, as follows:

$\mathrm{C}_{\text {Ts }}$ drag of the struts which hold the porous plug in place,

$C_{\text {Tn }}$ nonuniformities in the nozzle flow (flow not parallel to centerline or of unfform velocity),

$\mathrm{C}_{\mathrm{Tf}}$ boundary layer skin friction on the nozzle and plug,

$\mathrm{C}_{\mathrm{TP}}$ the normal pressures generated by flow separation on converging part of the plug, and

$\mathrm{C}_{\text {Th }}$ flow into and through the holes in the wall of the plug. The following estimates were developed for the various coefficients.

For $\mathrm{C}_{\mathrm{Ts}}$, we obtained

$$
\mathrm{C}_{\mathrm{Ts}}=0.0070 \text {, }
$$

which, of course, applies only to the particular strut and duct geometry used in the present tests. Secondly,

$$
\mathrm{C}_{\mathrm{Tn}}=0.0025
$$

for all the configurations except Configurations 2 and 3, for which 


$$
\mathrm{C}_{\mathrm{Tn}}=0.0050
$$

because of the converging-diverging geometry of Configurations 2 and 3 , whereas the other configurations have converging nozzles. The magnitude of these coefficients were based on an analysis of the $\mathrm{C}_{\mathrm{T}}$ data.

For $\mathrm{C}_{\mathrm{Tf}}$ we can perform an integration over the surfaces of the nozzle and plug, with the result

$$
c_{T f}=\frac{2}{d^{2}} \sum_{i=0}^{1} \int_{0}^{\ell} c_{f i} d_{i}\left(\frac{U_{i}}{U}\right)^{2} \cos \delta_{i} d_{i},
$$

where:

$$
\begin{aligned}
& i=0 \text { refers to the plug, } \\
& i=1 \text { refers to the nozzle, } \\
& \ell_{i} \quad \text { is the length of the plug or nozzle, } \\
& d_{i} \quad \text { is the local diameter, } \\
& C_{f i} \quad \text { is the skin friction coefficient (Reference 12), } \\
& U_{i} \quad \text { is the boundary layer edge velocity, and } \\
& \delta_{i} \text { is the angle between the wall and the nozzle centerline. }
\end{aligned}
$$

For the present calculations, $\mathrm{C}_{\mathrm{Tf}}$ was simplified by the approximation

$$
\mathrm{C}_{\mathrm{Tf}}=\frac{2}{\mathrm{~d}^{2}} \sum_{i=0}^{1} \mathrm{c}_{\mathrm{fi}} \mathrm{d}_{i} \ell_{\mathrm{ei}},
$$

where $\ell_{e i}$ is the "effective length" of the nozzle or plug. For the calculations, $\mathrm{C}_{\mathrm{f}}$ was taken to be 0.003 , based on Reference 14 and a typical Reynolds number of the turbulent boundary layer flow. The values of the ratios $d_{i} / d$ and $\ell_{e i} / d$ were assumed as given in Table 16 ; $l_{e o} / d$ is derivable from Table 1 as the sum

$$
\ell_{e o} / d=\ell_{c} / d+0.4\left(\ell-\ell_{c}\right) / d
$$

where the second term represents the tailcone portion of the plug. 
An expression was written to evaluate $\mathrm{C}_{\mathrm{Tp}}$, but the analysis of the $\mathrm{C}_{\mathrm{T}}$ data indicated that the pressure drag of the plugs was quite sma11; hence we have

$$
\mathrm{C}_{\mathrm{Tp}}=0
$$

Fina1ly, $\mathrm{C}_{\text {Th }}$ was put in the form

$c_{T h}=\frac{k_{h} \sigma d_{o} \ell_{e o}}{d^{2}}$

and the coefficient $K_{h}$ was evaluated, using the data, as

$$
\mathrm{K}_{\mathrm{h}}=0.07
$$




\section{APPENDIX D}

\section{EFFECTS OF FORWARD MOTION FLOW ON JET NOISE}

The acoustic effects of adding a forward-motion flow to a jet flowfield have been studied by Michel and Michalke, 15 and their predictions have shown excellent agreement with experiments. Their results for the "wind-tunnel" case where a jet with an Ideal jet speed $U_{1}$ is changed by the addition of an infinite forward motion flow with a speed $U_{f}$ give a change in the far-field sound pressure level that can be written as

$$
\Delta S P L_{f}=(10 \mathrm{~dB}) \log _{10}\left[\sigma_{1} \sigma^{2}\left(1+M_{f} \cos \theta_{o}\right)^{2}\left(\frac{U_{e}}{J_{o}}\right)^{m}\right]
$$

where the jet speed with respect to the nozzle, $U_{i}$, ts understood to be unchanged by the forward motion addition, and where

$$
\begin{aligned}
& U_{e}=\frac{U_{i}-U_{f}}{\left(1+M_{f} \cos \theta_{o}\right)} \\
& \sigma_{1}=\sigma=1+\frac{1.4 U_{f}}{U_{i}-U_{f}}
\end{aligned}
$$

$M_{f}=$ Mach number based on $U_{f}$

$\theta_{0}=$ acoustic emission angle

$\mathrm{m}=$ the velocity exponent defined by the equation

$$
\mathrm{m}=\frac{\mathrm{SPL}_{\mathrm{Ue}}-\mathrm{SPL}_{\mathrm{Ui}}}{(10 \mathrm{~dB}) \log _{10}\left(\frac{\mathrm{U}_{\mathrm{e}}}{\mathrm{U}_{\mathbf{i}}}\right)}
$$

where

$\mathrm{SPL}_{\mathrm{Ui}}=$ the sound pressure level without forward motion 


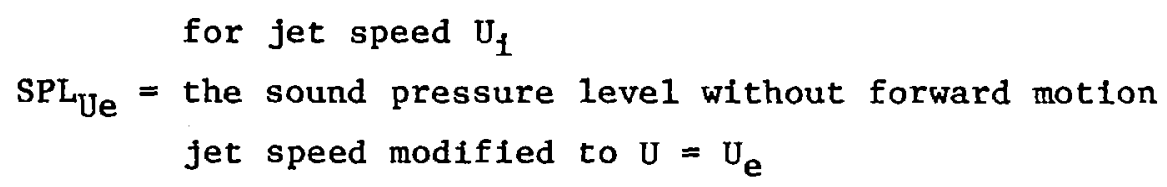

The above applies for sound pressure levels in one-third octave bands. The transformation from SPL $\mathrm{Ui}_{i}$ to $\mathrm{SPL}_{U \mathrm{Ue}}$ is accompanied by a band center frequency change by the factor

$$
\frac{f_{e}}{f_{i}}=\frac{U_{e}}{U_{1}}
$$

and the transformation of adding the forward motion to the jet is accompanied by a band center frequency change by the factor

$$
\frac{f_{f}}{f_{i}}=\sigma\left(1+M_{f} \cos \theta_{o}\right)\left(\frac{U_{e}}{U_{i}}\right)
$$

Note that Equation (D-1) gives the same result for all one-third octave bands. Hence, Equation (D-1) also applied to overall sound pressure levels. 


\begin{tabular}{|c|c|}
\hline \begin{tabular}{l|l} 
1. Report No. & 2. Government Accessic \\
NASA CR- 36.13 & \\
\end{tabular} & 3. Recipient's Catalog No. \\
\hline \multirow{2}{*}{$\begin{array}{l}\text { 4. Title and Subtitle } \\
\text { JET NOISE SUPPRESSION BY POROUS PLUG NOZZLES }\end{array}$} & \\
\hline & 6. Performing Organization Code \\
\hline $\begin{array}{l}\text { 7. Author(s) } \\
\text { A. B. Bauer, V. Kibens, and R. W. Wlezien }\end{array}$ & $\begin{array}{l}\text { 8. Performing Organization Report No. } \\
\text { MDC-J2475 }\end{array}$ \\
\hline \multirow{3}{*}{$\begin{array}{l}\text { 9. Performing Organization Name and Address } \\
\text { McDonnel1 Douglas Corporation } \\
\text { Douglas Aircraft Company } \\
\text { 3855 Lakewood Boulevard } \\
\text { Long Beach, CA } 90846\end{array}$} & 10. Work Unit No. \\
\hline & $\begin{array}{l}\text { 11. Contract or Grant No. } \\
\text { NAS } 1-16824\end{array}$ \\
\hline & 13. Type of Report and Period Covered \\
\hline \multirow{2}{*}{$\begin{array}{l}\text { 12. Sponsoring Agency Name and Address } \\
\text { National Aeronautics and Space Administration } \\
\text { Washington, D.C. } 20546\end{array}$} & Contractor Report \\
\hline & 14. Sponsoring Agency Code \\
\hline \multicolumn{2}{|l|}{$\begin{array}{l}\text { 15. Supplementary Notes } \\
\text { Langley Technical Monitor: S. Paul Pao } \\
\text { Final Report }\end{array}$} \\
\hline \multicolumn{2}{|c|}{$\begin{array}{l}\text { 16. Abstract } \\
\text { Jet noise suppression data presented earlier by Maestrello for porous plug nozzles have } \\
\text { been supplemented by the testing of a family of nozzles having an equivalent throat } \\
\text { diameter of } 11.77 \mathrm{~cm} \text {. Two circular reference nozzles and eight plug nozzles having } \\
\text { radius ratios of either } 0.53 \text { or } 0.80 \text { were tested at total pressure ratios of } 1.60 \text { to } \\
4.00 \text {. Data were taken both with and without a forward motion or coannular flow jet, } \\
\text { and some tests were made with a heated jet. Jet thrust was measured. The data were } \\
\text { analyzed to show the effects of suppressor geometry on nozzle propulsive efficiency and } \\
\text { jet noise. The noise reductions for subsonic pressure ratios were much less than those } \\
\text { for supersonic pressure ratios. Aerodynamic testing of the nozzles was carried out in } \\
\text { order to study the physical features that lead to the noise suppression. The aero- } \\
\text { dynamic flow phenomena were examined by the use of high-speed shadowgraph cinematography } \\
\text { still shadowgraphs, extensive static pressure probe measurements, and two-component } \\
\text { laser doppler velocimeter studies. The different measurement techniques correlated well } \\
\text { with each other and demonstrated that the porous plug changes the shock cell structure } \\
\text { of a standard nozzle into a series of smaller, periodic cell structures without strong } \\
\text { shock waves. These structures become smaller in dimension and have reduced pressure } \\
\text { variations as either the plug diameter or the porosity is increased, changes that also } \\
\text { reduce the jet noise and decrease thrust efficiency. As a result, a useful jet noise } \\
\text { porous plug suppressor is one which is a compromise between noise reduction and thrust } \\
\text { efficiency. }\end{array}$} \\
\hline
\end{tabular}

6. Abstract
Jet noise sup
been suppleme
diameter of
radius ratio
4.00 . Data
and some tes
analyzed to
jet noise.
for superson
order to stu
dynamic flow
still shadow
laser dopple
with each ot
f a standard
shock waves.
ariations a
reduce the
porous plug
efficiency.

18. Distribution Statement

17. Key Words (Suggested by Author(s))

FEDD Distribution

Nozzles, Thrust, Shadowgraphs, Velocimeter, Static pressure, Shock cells, Acoustics

Subject Category 71

19. Security Classif. (of this report)

Unclassifled
20. Security Classit. (of this page) Unclassified
21. No. of Pages

174 